\title{
A bestiary of black holes on the conifold with fluxes
}

\author{
Alex Buchel \\ Department of Applied Mathematics, Department of Physics and Astronomy, \\ University of Western Ontario, \\ London, ON N6A 5B\%, Canada \\ Perimeter Institute for Theoretical Physics, \\ Waterloo, ON N2J 2W9, Canada \\ E-mail: abuchel@uwo.ca
}

ABSTRACT: We present a comprehensive analysis of the black holes on warped deformed conifold with fluxes in Type IIB supergravity. These black holes realize the holographic dual to thermal states of the $\mathcal{N}=1$ supersymmetric $\mathrm{SU}(N) \times \mathrm{SU}(N+M)$ cascading gauge theory of Klebanov et al. [1,2] on round $S^{3}$. There are three distinct mass scales in the theory: the strong coupling scale $\Lambda$ of the cascading gauge theory, the compactification scale $\mu=1 / L_{3}$ (related to the $S^{3}$ radius $L_{3}$ ) and the temperature $T$ of a thermal state. Depending on $\Lambda, \mu$ and $T$, there is an intricate pattern of confinement/deconfinement (Hawking-Page) and the chiral symmetry breaking phase transitions. In the $S^{3} \rightarrow \mathbb{R}^{3}$ decompactification limit, i.e., $\mu \rightarrow 0$, we recover the Klebanov-Tseytlin [3] and the Klebanov-Strassler [4] black branes.

KEYwords: Gauge-gravity correspondence, Black Holes in String Theory, Holography and quark-gluon plasmas

ARXIV EPRINT: 2103.15188 


\section{Contents}

1 Introduction 1

2 Review: kS cascading gauge theory and its holographic dual 5

3 Review: type IIB supergravity on warped deformed conifold with fluxes 11

4 Vacua and black holes in $A d S_{5} \times T^{1,1} \quad 15$

$\begin{array}{lll}\text { 4.1 Conformal } \mathcal{V}_{A}^{s} \text { vacua } & 15\end{array}$

$\begin{array}{lll}\text { 4.2 Conformal } \mathcal{T}_{\text {decon }}^{s} \text { thermal states } & 18\end{array}$

5 Round $S^{\mathbf{3}}$ vacua of the warped deformed conifold with fluxes $\quad 20$

$\begin{array}{lll}5.1 & \mathcal{V}_{B} \text { vacua } & 25\end{array}$

$\begin{array}{lll}5.2 & \mathcal{V}_{A}^{s} \text { vacua } & 27\end{array}$

$\begin{array}{lll}5.3 \mathcal{V}_{A}^{b} \text { vacua } & 29\end{array}$

6 Black holes on the warped deformed conifold with fluxes 33

6.1 Conifold black branes and phase transitions at $\frac{\mu}{\Lambda}=0 \quad 33$

6.2 Conifold black holes and phase transitions at $\frac{\mu}{\Lambda} \neq 0 \quad 38$

$\begin{array}{lll}\text { 6.2.1 Canonical ensemble } & 38\end{array}$

6.2.2 Microcanonical ensemble 43

6.2.3 Black holes thermodynamics for select values of $\mu / \Lambda \neq 0 \quad 46$

6.3 Technical details on constructing the black holes, computing $T_{\chi \mathrm{SB}}(\mu)$ and $T_{c}(\mu) \quad 49$

6.3.1 Klebanov-Tseytlin black holes at $\mu \neq 0 \quad 50$

6.3.2 Computation of $T_{\chi \mathrm{SB}}(\mu) \quad 51$

6.3.3 Klebanov-Strassler black holes at $\mu \neq 0 \quad 53$

$\begin{array}{ll}\text { 6.3.4 Computation of } T_{c}(\mu) & 54\end{array}$

6.4 Conifold black holes at $\{T, \mu\} \gg \Lambda$

$\begin{array}{lll}7 & \text { Conclusion and open questions } & 59\end{array}$

A Equations of motion, asymptotic expansions, and the holographic renormalization

A.1 Case (A): horizonless warped deformed conifold with fluxes 60

A.1.1 Klebanov-Strassler solution [2] as $\mu \rightarrow 0$ limit of $\mathcal{V}_{B}$ conifold vacua 65

A.2 Case (B): schwarzschild horizon in warped deformed conifold with fluxes $\quad 66$

$\begin{array}{lll}\text { A.3 Holographic renormalization of the effective action (3.1) } & 70\end{array}$

$\begin{array}{lll}\text { A.4 Boundary stress-energy tensor } & 73\end{array}$

$\begin{array}{lll}\text { A.4.1 } \mathcal{V}_{A}^{b} \text { vacua } & 73\end{array}$

$\begin{array}{lll}\text { A.4.2 } \mathcal{V}_{A}^{s} \text { vacua } & 74\end{array}$

$\begin{array}{lll}\text { A.4.3 } \mathcal{V}_{B} \text { vacua } & 74\end{array}$ 
$\begin{array}{lll}\text { A.4.4 } \mathcal{T}_{\text {decon }}^{b} \text { thermal states } & 75\end{array}$

$\begin{array}{lll}\text { A.4.5 } & \mathcal{T}_{\text {decon }}^{s} \text { thermal states } & 75\end{array}$

$\begin{array}{llr}\text { B Fluctuations about } \mathcal{V}_{A}^{s} \text { vacua } & 76\end{array}$

$\begin{array}{llr}\text { C Fluctuations about } \mathcal{T}_{\text {decon }}^{s} \text { thermal states } & 77\end{array}$

$\begin{array}{ll}D \text { Conifold black holes at }\{T, \mu\} \gg \Lambda & 78\end{array}$

$\begin{array}{lr}\text { E Numerical tests } & \mathbf{8 0}\end{array}$

E.1 The first law of thermodynamics of $\mathcal{T}_{\text {decon }}^{s}$ and $\mathcal{T}_{\text {decon }}^{b}$ states at $\mu=\mu_{1} \quad 81$

E.2 Thermodynamics of $\mathcal{T}_{\text {decon }}^{s}$ states at $\mu=\mu_{2} \quad 82$

E.3 First law of thermodynamics of near-conformal conifold black holes 83

E.4 Additional numerical tests 84

\section{Introduction}

An exciting aspect of the gauge theory/string theory correspondence $[5,6]$ is that it geometrizes the confinement/deconfinement transitions of strongly coupled gauge theories as topology changing transitions in dual supergravity backgrounds. Following [7], consider $\mathcal{N}=4 \mathrm{SU}(N)$ supersymmetric Yang-Mills (SYM) theory on the 3 -sphere $S^{3}$ of radius $L_{3}$. In the planar limit, $N \rightarrow \infty$ and $g_{Y M}^{2} \rightarrow 0$ with $g_{Y M}^{2} N$ fixed, and for large 't Hooft coupling $N g_{Y M}^{2} \gg 1$, this SYM has a classical description as Type IIB supergravity on $A d S_{5} \times S^{5}$. Thermal states of the theory have two scales: the temperature $T$ and the $S^{3}$ compactification scale $\mu=\frac{1}{L_{3}}$. There are two distinct phases of the theory: the confined phase $\mathcal{T}_{\text {con }}$ and the deconfined phase $\mathcal{T}_{\text {decon }}$. In the large- $N$ limit there is a sharp distinction between the two phases: the confined phase has an entropy $s \propto O\left(N^{0}\right)$, while the deconfined phase has an entropy $s \propto O\left(N^{2}\right)$. The former one is represented gravitationally as $E A d S_{5} \times S^{5}$ with the Euclidean time direction $t_{E}$ compactified as

$$
t_{E} \sim t_{E}+\frac{1}{T}
$$

and the latter one as a black hole in global $A d S_{5}$. Which phase is the preferred one, depends on the ratio $T / \mu$ : when $T \gg \mu$, the gravitational free energy of the black hole solution is lower compare to $E A d S_{5}$, and the deconfined phase is the preferred one. The confined phase is the preferred one at low temperatures. On the gravity side of the holographic duality, the confinement/deconfinement transition is the Hawking-Page thermal transition in anti-de Sitter space-time [8]. Restricted to $E A d S_{5}$, the two thermal phases are shown in figure 1 . These are the only $\mathrm{SO}(4) \times \mathrm{SO}(6)$ symmetric thermal phases of the SYM.

In this paper we study vacua and black holes ${ }^{1}$ on the conifold [9] with fluxes in Type IIB supergravity. These gravitational backgrounds are holographic duals to the vacua and the thermal states of $\mathcal{N}=1 \mathrm{SU}(N+M) \times \mathrm{SU}(N)$ cascading gauge theory [2] on $S^{3}$.

\footnotetext{
${ }^{1}$ The black holes studied are Schwarzschild-AdS-like, namely they carry no angular momentum or electric charge.
} 
$\mathcal{T}_{\text {con }}$

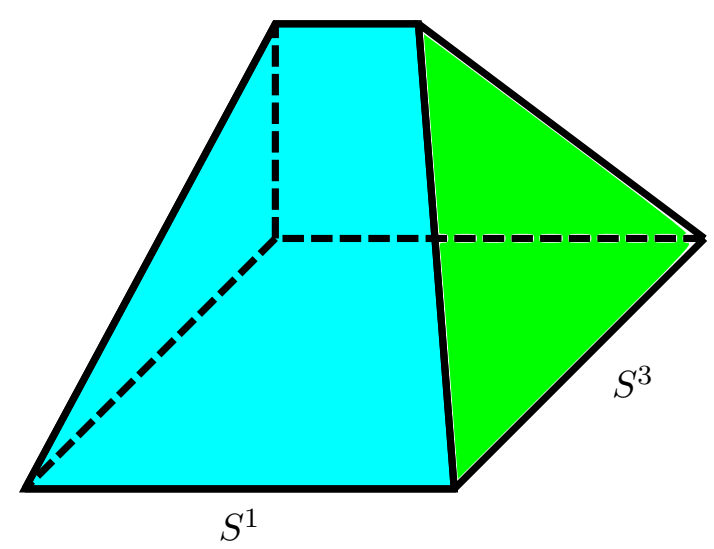

$\mathcal{T}_{\text {decon }}$

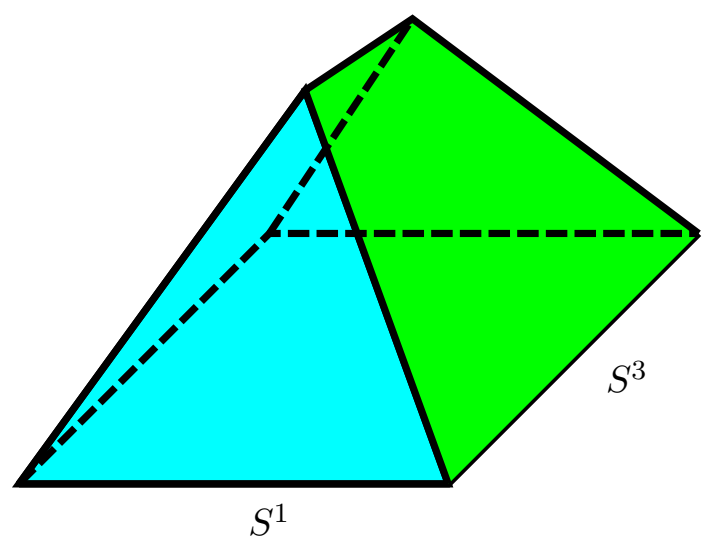

Figure 1. Geometrical phases in global $E A d S_{5}$ : the cyan face represents Euclidean time direction $S^{1}$, compactified with the period $\frac{1}{T}$, the green face represents the 3 -sphere $S^{3}$. The bulk geometry with the $S^{3}$ smoothly shrinking to zero size represents the confined phase of thermal $\mathcal{N}=4 \mathrm{SYM}$ (the left panel). The bulk geometry with the $S^{1}$ smoothly shrinking to zero size represents the deconfined phase of thermal $\mathcal{N}=4 \mathrm{SYM}$ (the right panel).

$\mathcal{V}_{A}^{S}$

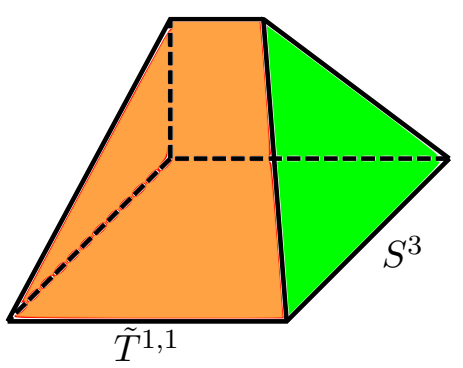

$\mathcal{V}_{B}$

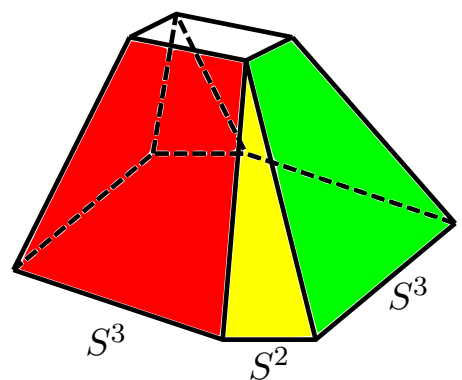

$\mathcal{V}_{A}^{b}$

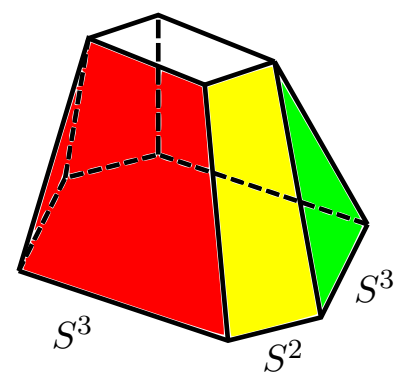

Figure 2. Vacua of the cascading gauge theory on $S^{3}$ (the green faces) are characterized by topologically distinct gravitational backgrounds: in the left panel the boundary $S^{3}$ shrinks to zero size, with the internal $\tilde{T}^{1,1}$ (orange face) of the warped conifold having unbroken U(1) symmetry; in the middle panel the 2-cycle (the yellow faces) of the deformed conifold shrinks to zero size; in the right panel the boundary $S^{3}$ shrinks to zero size but the conifold is deformed — the U(1) symmetry of $\tilde{T}^{1,1}$ is spontaneously broken to $\mathbb{Z}_{2}$.

Unlike $\mathcal{N}=4$ SYM, the cascading gauge is not conformal, and has a strong coupling scale $\Lambda$. Thus, already the vacua of the theory are characterized by two mass scales $\Lambda$ and $\mu$, leading to a nontrivial phase structure. In figure 2 we characterize distinct vacua of the compactified cascading gauge theory according to the topology of the gravitational dual:

- $\mathcal{V}_{A}^{s}$ - vacua with the boundary $S^{3}$ (akin the 3 -sphere in the $\mathcal{N}=4$ discussion above) smoothly shrinking to zero size (the left panel). In these vacua the chiral symmetry of the gauge theory is unbroken. 

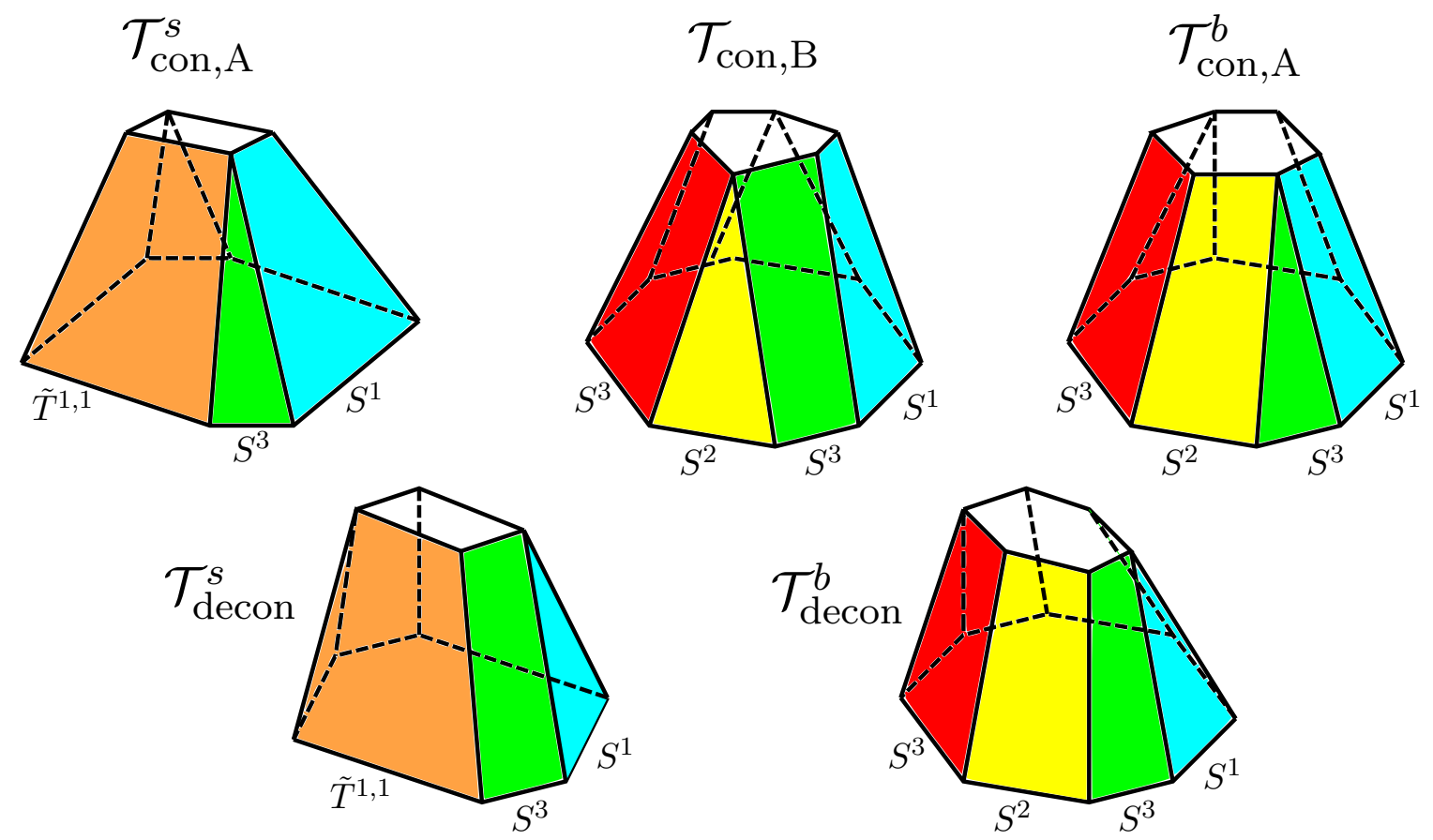

Figure 3. Top row: confined thermal states of the cascading gauge theory, obtained from the compactification of the Euclidean time direction as in (1.1) of the corresponding vacua, see figure 2. Bottom row, left panel: deconfined thermal states with the unbroken U(1) symmetry - the Klebanov-Tseytlin black holes. Bottom row, right panel: deconfined thermal states with the U(1) symmetry spontaneously broken to $\mathbb{Z}_{2}$ — the Klebanov-Strassler black holes.

- $\mathcal{V}_{B}$ - vacua with the conifold 2-cycle $S^{2}$ smoothly shrinking to zero size (the middle panel). In these vacua the chiral symmetry of the gauge theory is spontaneously broken.

- $\mathcal{V}_{A}^{b}$ - vacua with the boundary $S^{3}$ (akin the 3 -sphere in the $\mathcal{N}=4$ discussion above) smoothly shrinking to zero size (the right panel). In these vacua the chiral symmetry of the gauge theory is spontaneously broken.

We discuss different vacua of the cascading gauge theory and the phase transitions in section 5 .

The set of possible thermal states of the cascading gauge theory is yet richer: in addition to $\Lambda$ and $\mu$ we have the temperature $T$. In figure 3 we characterize distinct vacua of the compactified cascading gauge theory according to the topology of the gravitational dual:

- $\mathcal{T}_{\text {con, A }}^{s}$ - confined thermal states with the boundary $S^{3}$ smoothly shrinking to zero size (the top row, left panel). In these states the chiral symmetry of the gauge theory is unbroken. These thermal states are the Euclidean $\mathcal{V}_{A}^{s}$ vacua with the compactified time direction as in (1.1).

- $\mathcal{T}_{\text {con,B }}$ - confined thermal states with the conifold 2-cycle $S^{2}$ smoothly shrinking to zero size. In these vacua the chiral symmetry of the gauge theory is spontaneously 
broken (the top row, middle panel). These thermal states are the Euclidean $\mathcal{V}_{B}$ vacua with the compactified time direction as in (1.1).

- $\mathcal{T}_{\text {con, A }}^{b}$ - confined thermal states with the boundary $S^{3}$ smoothly shrinking to zero size (the top row, right panel). In these states the chiral symmetry of the gauge theory is spontaneously broken. These thermal states are the Euclidean $\mathcal{V}_{A}^{b}$ vacua with the compactified time direction as in (1.1).

- $\mathcal{T}_{\text {decon }}^{S}$ - deconfined thermal states with the Euclidean time direction $S^{1}$ (cyan faces) smoothly shrinking to zero size (the bottom row, left panel). In these states the chiral symmetry of the gauge theory is unbroken. We call these thermal states the Klebanov-Tseytlin black holes.

- $\mathcal{T}_{\text {decon }}^{b}$ - deconfined thermal states with the Euclidean time direction $S^{1}$ smoothly shrinking to zero size (the bottom row, right panel). In these states the chiral symmetry of the gauge theory is spontaneously broken. We call these thermal states the Klebanov-Strassler black holes.

We discuss different thermal states of the cascading gauge theory and the phase transitions in section 6 .

The black branes on the conifold were studied previously in $[3,4]$ - these solutions can be obtained as the $\mu / \Lambda \rightarrow 0$, i.e., the $S^{3}$ decompactification, limit of the black holes analyzed here. $\mathcal{V}_{A}^{s}$ and $\mathcal{V}_{B}$ vacua of the conifold with fluxes were analyzed earlier in [10]. $\mathcal{V}_{A}^{b}$ vacua are constructed here for the first time.

The rest of the manuscript is organized as follows. To have a self-contained presentation, in section 2 we review the cascading gauge theory and its holographic dual [2]. We present an overview of how the cascading gauge theory fits into the framework of the topdown holography, and what is precisely the gravitational dual to the strong coupling scale $\Lambda$ of the theory. In section 3 we review the consistent truncation in $\mathrm{SU}(2) \times \mathrm{SU}(2) \times \mathbb{Z}_{2}$ invariant sector of Type IIB supergravity on warped deformed conifold with fluxes to an arbitrary five dimensional manifold $\mathcal{M}_{5}$ [11]. This effective five-dimensional gravitational action is our holographic dual to cascading gauge theory on $\mathcal{M}_{4} \equiv \partial \mathcal{M}_{5}$. In section 4 we use the framework of the effective action of section 3 to reproduce the known results about $\mathcal{N}=1$ Klebanov-Witten superconformal gauge theory holography [12]. The purpose is to gently familiarize the reader with the notations, and the holographic renormalization of the conifold effective action. Sections 5 and 6 collect the main results of this paper. For the most part, we tried in these sections to make the presentation as free from the technicalities as practical. Interested reader can find technical guidance in section 6.3, and more complete assembly of relevant formulas in the appendices. We conclude, mainly listing the open problems, in section 7. Extensive numerical tests conducted are covered in appendix E.

Numerical work described in this paper has been done using Maplesoft ${ }^{\circ}$ (symbolic manipulations) and Wolfram Mathematica ${ }^{0}$ (numerics). Unfortunately, it is not practical to publicly release the code and the data produced, as it takes over $150 \mathrm{MB}$. 


\section{Review: kS cascading gauge theory and its holographic dual}

Following [13-15], we present a brief review of the cascading gauge theory and its holographic dual [2].

The starting point is the original Maldacena duality [5] between $\mathcal{N}=4 \mathrm{SU}(N)$ supersymmetric Yang-Mills theory (SYM) and Type IIB supergravity on $A d S_{5} \times S^{5}$ :

$$
d s_{10}^{2}=\frac{r^{2}}{L^{2}}\left(-d t^{2}+d x_{i}^{2}\right)+\frac{L^{2}}{r^{2}} d r^{2}+L^{2}\left(d S^{5}\right)^{2}
$$

there is also a constant dilaton $e^{\Phi}=g_{s}$, and a quantized self-dual R-R five form $F_{5}=$ $\mathcal{F}_{5}+\star \mathcal{F}_{5}$,

$$
\mathcal{F}_{5}=16 \pi\left(\alpha^{\prime}\right)^{2} N \operatorname{vol}\left(S^{5}\right), \quad \frac{1}{\left(4 \pi^{2} \alpha^{\prime}\right)^{2}} \int_{S^{5}} \mathcal{F}_{5}=N \in \mathbb{Z} .
$$

The radii of $A d S_{5}$ and $S^{5}$ are

$$
L^{4}=4 \pi g_{s} N\left(\alpha^{\prime}\right)^{2}
$$

The string coupling $g_{s}$ is related to the exactly marginal coupling $g_{Y M}$ of $\mathcal{N}=4 \mathrm{SYM}$ as

$$
g_{Y M}^{2}=4 \pi g_{s} .
$$

This basic AdS/CFT correspondence, restricted as above to the supergravity approximation and to leading order in $\alpha^{\prime}$, implies the planar limit on the SYM size, namely $N \rightarrow \infty$ with $g_{Y M}^{2} N$ kept fixed, and for the large 't Hooft coupling constant, i.e., $g_{Y M}^{2} N \gg 1$. In this paper we always work in the two-derivative Type IIB supergravity approximation.

$\mathcal{N}=4 \mathrm{SYM}$ is a conformal theory with central charges $c=a^{2}$

$$
c=\left.a\right|_{\mathcal{N}=4 S Y M}=\frac{N^{2}}{4} .
$$

The central charges characterize the quantum anomaly in the trace of the energy momentum tensor of the theory on $\mathcal{M}_{4}$,

$$
\left\langle T_{\mu}^{\mu}\right\rangle=\frac{1}{(4 \pi)^{2}}\left(c I_{4}-a E_{4}+b \square R\right)
$$

where the four-dimensional Euler density $E_{4}$ and the Weyl curvature $I_{4}$ are given by

$$
E_{4}=R_{\mu \nu \rho \lambda} R^{\mu \nu \rho \lambda}-4 R_{\mu \nu} R^{\mu \nu}+R^{2}, \quad I_{4}=R_{\mu \nu \rho \lambda} R^{\mu \nu \rho \lambda}-2 R_{\mu \nu} R^{\mu \nu}+\frac{1}{3} R^{2},
$$

and $b$ is the renormalization scheme dependent constant. They determine the Casimir energy $E_{0}$ of the vacuum state of the theory on $\mathcal{M}_{4}$. The Casimir energy is renormalization scheme-dependent [16] — it depends on finite counterterms in the effective action, which basis can be taken to be $E_{4}, I_{4}$, the Pontryagin density $\operatorname{Tr}(R \wedge R)$ and $R^{2}$. In this paper

\footnotetext{
${ }^{2}$ In the supergravity approximation the two central charges are the same for any dual pair in the gauge/gravity correspondence.
} 
we will be interested in gauge theories on $\mathcal{M}_{4}=R \times S^{3}$, and it is only the counterterm due to $R^{2}$ that is nonvanishing:

$$
S_{\text {ct }}^{\mathrm{finite}}=-\frac{b}{12(4 \pi)^{2}} \int_{\mathcal{M}_{4}} \operatorname{vol}_{\mathcal{M}_{4}} R^{2},
$$

where the scheme-dependent coefficient $b$ correlates with the trace anomaly ambiguity in (2.6). The finite counterterm (2.8) leads to an ambiguity $\delta E_{0}$ of the SYM on the $S^{3}$ of radius $\frac{1}{\mu}$ as

$$
\delta E_{0}=\frac{3 b}{8} \mu .
$$

While we discussed here the Casimir energy ambiguity of the conformal theory, it is clear that thermal states of the conformal theory on $S^{3}$ have ambiguous energy density $\mathcal{E}$ and the pressure $\mathcal{P}$ as well. Specifically, corresponding to (2.8), we have renormalization scheme ambiguities leading to

$$
\delta \mathcal{E}=\frac{3 b}{16 \pi^{2}} \mu^{4}, \quad \delta \mathcal{P}=\frac{1}{3} \delta \mathcal{E} .
$$

The next step towards construction of the cascading gauge theory is to $\mathbb{Z}_{2}$ orbifold $\mathcal{N}=4 \mathrm{SU}(N)$ SYM. The resulting [17] $\mathcal{N}=2 \mathrm{SU}(N) \times \mathrm{SU}(N)$ superconformal quiver gauge theory has hypermultiplets transforming in $(N, \bar{N}) \oplus(\bar{N}, N)$ representation. From the $\mathcal{N}=1$ point of view, the hypermultiplets correspond to pairs of chiral multiplets $A_{k}, B_{\ell}, k, \ell=1,2$ in the $(N, \bar{N})$ and $(\bar{N}, N)$ representations correspondingly. There are additional $\mathcal{N}=1$ chiral multiples $\Phi$ and $\tilde{\Phi}$ in the adjoint representations of the two gauge group factors. The theory has a superpotential

$$
g_{Y M} \operatorname{Tr} \Phi\left(A_{1} B_{1}+A_{2} B_{2}\right)+g_{Y M} \operatorname{Tr} \tilde{\Phi}\left(B_{1} A_{1}+B_{2} A_{2}\right),
$$

and $\mathrm{SU}(2) \times \mathrm{SU}(2)$ global flavor symmetry associated with rotations of $A_{k}$ and $B_{\ell}$. The holographic dual of the orbifold model was identified as $A d S_{5} \times S^{5} / \mathbb{Z}_{2}$ orbifold in [18]. Locally, the dual Type IIB background metric is as in (2.1); because of the orbifolding the central charges are

$$
c=\left.a\right|_{S^{5} / \mathbb{Z}_{2} \text { orbifold }}=\frac{N^{2}}{2}
$$

leading to a modified expression for the $A d S_{5}$ radius $L$ :

$$
L^{4}=4 \pi g_{s} N\left(\alpha^{\prime}\right)^{2} \frac{\operatorname{vol}\left(S^{5}\right)}{\operatorname{vol}\left(S^{5} / \mathbb{Z}_{2}\right)}=8 \pi g_{s} N\left(\alpha^{\prime}\right)^{2} .
$$

In [12] (KW) the authors discussed an important holographic RG flow from the above orbifold model in the UV. Specifically, they added to a superpotential (2.11) a relevant term

$$
\frac{m}{2}\left(\operatorname{Tr} \Phi^{2}-\operatorname{Tr} \tilde{\Phi}^{2}\right) \text {. }
$$

This mass term explicitly breaks the conformal invariance, and the supersymmetry to $\mathcal{N}=1$. Below the energy scale set by $m$, the adjoint chiral multiples can be integrated out leading to the superpotential

$$
\frac{g_{Y M}^{2}}{2 m}\left[\operatorname{Tr}\left(A_{1} B_{1} A_{2} B_{2}\right)-\operatorname{Tr}\left(B_{1} A_{1} B_{2} A_{2}\right)\right] .
$$


It was argued in [12] that the mass-deformed orbifold model flows in the IR to $\mathcal{N}=1$ superconformal fixed point. Using the $\mathrm{U}(1)_{R}$ symmetry of the superconformal fixed point, and the $\mathrm{SU}(2) \times \mathrm{SU}(2)$ global symmetry, the anomalous dimensions of $A_{k}$ and $B_{\ell}$ chiral superfields can be computed as

$$
\gamma_{A_{k}}=\gamma_{B_{\ell}}=-\frac{1}{4}
$$

resulting in an exactly marginal superpotential in the IR:

$$
\mathcal{W}_{K W}=\frac{\lambda}{2} \epsilon^{i j} \epsilon^{k \ell} \operatorname{Tr} A_{i} B_{k} A_{j} B_{\ell} .
$$

The central charges $\left.a\right|_{K W}$ and $\left.c\right|_{K W}$ of the IR superconformal fixed point were determined in [19]; in the large- $N$ limit,

$$
\frac{\left.a\right|_{K W}}{\left.a\right|_{S^{5} / \mathbb{Z}_{2} \text { orbifold }}}=\frac{\left.c\right|_{K W}}{\left.c\right|_{S^{5} / \mathbb{Z}_{2} \text { orbifold }}}=\frac{27}{32} \quad \Longrightarrow \quad c=\left.a\right|_{K W}=\frac{27 N^{2}}{64} .
$$

The holographic dual to the full gauge theory renormalization group flow described above is currently unknown. The end point of the RG flow was argued to be holographically dual to a near-horizon geometry of D3 branes on a singular conifold [9] — this is commonly referred to as a Klebanov-Witten model [12]. Here, the background geometry is

$$
d s_{10}^{2}=\frac{r^{2}}{L^{2}}\left(-d t^{2}+d x_{i}^{2}\right)+\frac{L^{2}}{r^{2}} d r^{2}+L^{2} d s_{T^{1,1}}^{2}
$$

where

$$
d s_{T^{1,1}}^{2}=\frac{1}{9}\left(d \psi+\sum_{i=1}^{2} \cos \theta_{i} d \phi_{i}\right)^{2}+\frac{1}{6} \sum_{i=1}^{2}\left(d \theta_{i}^{2}+\sin ^{2} \theta_{i} d \phi_{i}^{2}\right)
$$

is the metric on $T^{1,1} \equiv(\mathrm{SU}(2) \times \mathrm{SU}(2)) / \mathrm{U}(1)$ coset (the base of the conifold). The angular coordinates range as $0 \leq \psi \leq 4 \pi, 0 \leq \theta_{a} \leq \pi$ and $0 \leq \phi_{a} \leq 2 \pi(a=1,2)$. Anticipating the generalization to the deformed conifold [9], we introduce the following basis of one-forms on the compact space [20]:

$$
g_{1}=\frac{\alpha^{1}-\alpha^{3}}{\sqrt{2}}, \quad g_{2}=\frac{\alpha^{2}-\alpha^{4}}{\sqrt{2}}, \quad g_{3}=\frac{\alpha^{1}+\alpha^{3}}{\sqrt{2}}, \quad g_{4}=\frac{\alpha^{2}+\alpha^{4}}{\sqrt{2}}, \quad g_{5}=\alpha^{5},
$$

where

$$
\begin{aligned}
& \alpha^{1}=-\sin \theta_{1} d \phi_{1}, \quad \alpha^{2}=d \theta_{1}, \quad \alpha^{3}=\cos \psi \sin \theta_{2} d \phi_{2}-\sin \psi d \theta_{2}, \\
& \alpha^{4}=\sin \psi \sin \theta_{2} d \phi_{2}+\cos \psi d \theta_{2}, \quad \alpha^{5}=d \psi+\cos \theta_{1} d \phi_{1}+\cos \theta_{2} d \phi_{2},
\end{aligned}
$$

allowing to rewrite the metric (2.19) as

$$
d s_{10}^{2}=\frac{r^{2}}{L^{2}}\left(-d t^{2}+d x_{i}^{2}\right)+\frac{L^{2}}{r^{2}} d r^{2}+L^{2}\left(\frac{1}{9} g_{5}^{2}+\frac{1}{6}\left(g_{3}^{2}+g_{4}^{2}\right)+\frac{1}{6}\left(g_{1}^{2}+g_{2}^{2}\right)\right) .
$$

Besides the metric, Type IIB supergravity background includes a constant string coupling $g_{s}$ and a self-dual R-R five form $F_{5}=\mathcal{F}_{5}+\star \mathcal{F}_{5}$,

$$
\mathcal{F}_{5}=27 \pi\left(\alpha^{\prime}\right)^{2} N \operatorname{vol}\left(T^{1,1}\right), \quad \frac{1}{\left(4 \pi^{2} \alpha^{\prime}\right)^{2}} \int_{T^{1,1}} \mathcal{F}_{5}=N \in \mathbb{Z} .
$$


The $A d S_{5}$ and $T^{1,1}$ radii $L$ are given by

$$
L^{4}=4 \pi g_{s} N\left(\alpha^{\prime}\right)^{2} \frac{\operatorname{vol}\left(S^{5}\right)}{\operatorname{vol}\left(T^{1,1}\right)}=\frac{27}{4} \pi g_{s} N\left(\alpha^{\prime}\right)^{2} .
$$

The holographic computation of the boundary stress-energy trace anomaly reproduces the central charge (2.18), see [21].

The final step in constructing the cascading gauge theory is to deform the $\mathcal{N}=1$ Klebanov-Witten superconformal gauge theory by shifting the rank of one of the gauge groups [2]: $\mathrm{SU}(N+M) \times \mathrm{SU}(N)$, keeping pairs of chiral multiplets $A_{k}$ and $B_{\ell}, k, \ell=1,2$, in the bifundamental $(N+M, \bar{N})$ and $(\overline{N+N}, N)$ representations, and the superpotential as in (2.17). When $M \neq 0$, the theory is no longer conformal, and the gauge couplings $g_{1}$ and $g_{2}$, of the gauge group factors $\mathrm{SU}(N+M)$ and $\mathrm{SU}(N)$ correspondingly, run with the renormalization group scale $\hat{\mu}$,

$$
\begin{aligned}
& \frac{d}{d \ln (\hat{\mu} / \Lambda)} \frac{8 \pi^{2}}{g_{1}^{2}}=3(N+M)-2 N(1-\gamma), \\
& \frac{d}{d \ln (\hat{\mu} / \Lambda)} \frac{8 \pi^{2}}{g_{2}^{2}}=3 N-2(N+M)(1-\gamma),
\end{aligned}
$$

where $\gamma$ is the anomalous dimension of operators $\operatorname{Tr} A_{i} B_{j}$ and $\Lambda$ is the strong coupling scale of the cascading gauge theory. ${ }^{3}$ To leading order in $M / N, \gamma=-\frac{1}{2}$ (see (2.16)), so that

$$
\frac{8 \pi^{2}}{g_{1}^{2}}-\frac{8 \pi^{2}}{g_{2}^{2}}=6 M \ln \frac{\hat{\mu}}{\Lambda} \times(1+\mathcal{O}(M / N)),
$$

while the sum of the gauge couplings is constant along the RG flow

$$
\frac{8 \pi^{2}}{g_{1}^{2}}+\frac{8 \pi^{2}}{g_{2}^{2}}=\text { const } \equiv \frac{2 \pi}{g_{s}} .
$$

An immediate consequence of (2.27) and (2.28) is that as one goes to the UV ( $\hat{\mu}$ increases) $g_{2}$ diverges at some finite value of $\hat{\mu}=\hat{\mu}_{U V} \approx \Lambda e^{\frac{\pi}{3 M g_{s}}}$; while as one go to the IR $(\hat{\mu}$ decreases) $g_{1}$ diverges at some finite value of $\hat{\mu}=\hat{\mu}_{I R} \approx \Lambda e^{-\frac{\pi}{3 M g_{s}}}$. To continue the RG flow past $\hat{\mu}_{U V}$ or $\hat{\mu}_{I R}$, we must perform $\mathcal{N}=1$ gauge theory Seiberg duality [22]. For a cascading gauge theory discussed, Seiberg duality is a self-similar transformation of the theory leading to $N \rightarrow N+M$ for $\hat{\mu}>\hat{\mu}_{U V}$, and $N \rightarrow N-M$ for $\hat{\mu}<\hat{\mu}_{I R}$ [2]. Effectively, the rank $N$ of the cascading gauge theory runs along the RG flow as [23]

$$
N=N(\hat{\mu}) \propto+M^{2} \ln \frac{\hat{\mu}}{\Lambda} .
$$

Although the duality cascade extends indefinitely in the UV, it stops in the IR since the negative values of $N(\hat{\mu})$ are nonphysical. In general, the IR structure of the cascading gauge theory can be rather involved [24]; when $N$ is an integer multiple of $M$, the cascading gauge theory ends up in the IR as the $\mathcal{N}=1 \mathrm{SU}(M)$ Yang-Mills theory. It confines with a spontaneous breaking of the $\mathrm{U}(1)_{R}$ chiral symmetry,

$$
\mathrm{U}(1)_{R} \rightarrow \mathbb{Z}_{2}
$$

\footnotetext{
${ }^{3}$ Below, we use the holographic dual to provide a precise non-perturbative definition of $\Lambda$.
} 
The holographic dual of 'shifting the rank of one of the gauge groups' of the KW superconformal gauge theory is to supplement the $N$ D3 branes at the tip of the conifold with $M$ D5 branes wrapping the vanishing 2-cycle of the conifold. The resulting Type IIB supergravity background takes form [2]

$$
d s_{10}^{2}=H_{K S}^{-1 / 2}\left(-d t^{2}+d x_{i}^{2}\right)+\Omega_{1}^{2}\left(d \tau^{2}+g_{5}^{2}\right)+\Omega_{2}^{2}\left(g_{3}^{2}+g_{4}^{2}\right)+\Omega_{3}^{2}\left(g_{1}^{2}+g_{2}^{2}\right),
$$

where

$$
\begin{aligned}
& \Omega_{i}=\omega_{i, K S} H_{K S}^{1 / 4}, \quad \omega_{1, K S}=\frac{\epsilon^{2 / 3}}{\sqrt{6} K}, \quad \omega_{2, K S}=\frac{\epsilon^{2 / 3} K^{1 / 2}}{\sqrt{2}} \cosh \frac{\tau}{2}, \\
& \omega_{3, K S}=\frac{\epsilon^{2 / 3} K^{1 / 2}}{\sqrt{2}} \sinh \frac{\tau}{2}, \quad K=\frac{(\sinh (2 \tau)-2 \tau)^{1 / 3}}{2^{1 / 3} \sinh \tau}, \\
& \frac{d}{d \tau} H_{K S}=8 \frac{\left(2 h_{2, K S}-M \alpha^{\prime}\right) h_{1, K S}-2 h_{2, K S} h_{3, K S}}{\epsilon^{8 / 3} K^{2} \sinh ^{2} \tau},
\end{aligned}
$$

with

$$
\begin{aligned}
& h_{1, K S}=\frac{M \alpha^{\prime} g_{s}}{4} \frac{\cosh \tau-1}{\sinh \tau}\left(\frac{\tau \cosh \tau}{\sinh \tau}-1\right), \quad h_{2, K S}=\frac{M \alpha^{\prime} g_{s}}{4}\left(1-\frac{\tau}{\sinh \tau}\right), \\
& h_{3, K S}=\frac{M \alpha^{\prime} g_{s}}{4} \frac{\cosh \tau+1}{\sinh \tau}\left(\frac{\tau \cosh \tau}{\sinh \tau}-1\right),
\end{aligned}
$$

for the Einstein frame metric with a radial coordinate $\tau \in[0, \infty)$. The asymptotic boundary is as $\tau \rightarrow+\infty$, while the deformed conifold 2-cycle collapsing as

$$
\propto \epsilon^{4 / 3} \frac{\tau^{2}}{4}\left(g_{1}^{2}+g_{2}^{2}\right)
$$

as $\tau \rightarrow 0$, with the 3 -cycle remaining finite

$$
\propto \epsilon^{4 / 3}\left(\frac{1}{2} g_{5}^{2}+g_{3}^{2}+g_{4}^{2}\right) .
$$

The dilaton is constant,

$$
e^{\Phi}=g_{s},
$$

R-R 3-form flux $F_{3}=F_{3}^{\text {top }}+d C_{2}$ and NS-NS 3-form flux $H_{3}=d B_{2}$ are

$$
\begin{aligned}
B_{2} & =h_{1, K S} g_{1} \wedge g_{2}+h_{3, K S} g_{3} \wedge g_{4}, \quad C_{2}=h_{2, K S}\left(g_{1} \wedge g_{3}+g_{2} \wedge g_{4}\right), \\
F_{3}^{\text {top }} & =\frac{1}{2} M \alpha^{\prime} g_{5} \wedge g_{3} \wedge g_{4},
\end{aligned}
$$

and the self-dual R-R five-form $F_{5}=\mathcal{F}_{5}+\star \mathcal{F}_{5}$,

$$
g_{s} \mathcal{F}_{5}=B_{2} \wedge F_{3}=\left(h_{2, K S}\left(h_{3, K S}-h_{1, K S}\right)+\frac{1}{2} M \alpha^{\prime} h_{1, K S}\right) g_{1} \wedge g_{2} \wedge g_{3} \wedge g_{4} \wedge g_{5} .
$$

KS solution has 3 parameters:

- $g_{s}$ - the string coupling, related to the RG invariant sum of the cascading gauge theory coupling constants (2.28); 
- an integer $M$ defining the topological part of the R-R 3-form flux $F_{3}^{\text {top }}$ (2.37) (equivalently the number of D5 branes wrapping the conifold 2-cycle), related to the difference of the ranks of the cascading gauge theory groups;

- the conifold complex structure deformation parameter $\epsilon$, determining the strong coupling scale $\Lambda$ of the cascading gauge theory and mass scale in the glueball spectrum

$$
\Lambda=\frac{2^{5 / 12} e^{1 / 3}}{3^{3 / 2}} \frac{\epsilon^{2 / 3}}{M g_{s}^{1 / 2} \alpha^{\prime}}, \quad m_{\text {glueball }} \equiv \frac{\epsilon^{2 / 3}}{M g_{s} \alpha^{\prime}},
$$

where the specific numerical prefactor in the definition of the strong coupling scale is chosen to agree ${ }^{4}$ with the earlier computations of thermodynamics of the cascading gauge theory on $\mathbb{R}^{3}$.

To facilitate the identification of the strong coupling scale in the numerical solutions representing the cascading gauge theory on $S^{3}$ discussed in sections $5-6$, we rewrite the KS solution (2.32)-(2.38) in a radial coordinate $\rho$ defined as in (3.15). Following [15] (see appendix B.3 there), from

$$
\frac{(d \rho)^{2}}{\rho^{4}}=\left(\omega_{1, K S}(\tau)\right)^{2}(d \tau)^{2}
$$

and introducing

$$
z \equiv e^{-\tau / 3}
$$

we find

$$
\frac{1}{\rho}=\frac{\sqrt{6}(2 \epsilon)^{2 / 3}}{4} \int_{1}^{z} d u \frac{u^{6}-1}{u^{2}\left(1-u^{12}+12 u^{6} \ln u\right)^{1 / 3}} .
$$

In the UV, $\tau \rightarrow \infty, z \rightarrow 0$ and $\rho \rightarrow 0$ we have

$$
\begin{aligned}
& e^{-\tau / 3} \equiv z=\frac{\sqrt{6}(2 \epsilon)^{2 / 3}}{4} \rho\left(1+\mathcal{Q} \rho+\mathcal{Q}^{2} \rho^{2}+\mathcal{Q}^{3} \rho^{3}+\mathcal{Q}^{4} \rho^{4}+\mathcal{Q}^{5} \rho^{5}+\left(\frac{27}{80} \epsilon^{4} \ln 3+\mathcal{Q}^{6}\right.\right. \\
& \left.+\frac{27}{800} \epsilon^{4}-\frac{9}{16} \epsilon^{4} \ln 2+\frac{9}{20} \epsilon^{4} \ln \epsilon+\frac{27}{40} \epsilon^{4} \ln \rho\right) \rho^{6}+\left(-\frac{63}{16} \epsilon^{4} \mathcal{Q} \ln 2+\frac{189}{80} \epsilon^{4} \mathcal{Q} \ln 3+\mathcal{Q}^{7}\right. \\
& \left.+\frac{729}{800} \mathcal{Q} \epsilon^{4}+\frac{63}{20} \epsilon^{4} \mathcal{Q} \ln \epsilon+\frac{189}{40} \mathcal{Q} \epsilon^{4} \ln \rho\right) \rho^{7}+\left(\frac{2403}{400} \epsilon^{4} \mathcal{Q}^{2}-\frac{63}{4} \epsilon^{4} \mathcal{Q}^{2} \ln 2+\frac{189}{20} \epsilon^{4} \mathcal{Q}^{2} \ln 3\right. \\
& \left.+\frac{63}{5} \epsilon^{4} \mathcal{Q}^{2} \ln \epsilon+\mathcal{Q}^{8}+\frac{189}{10} \epsilon^{4} \mathcal{Q}^{2} \ln \rho\right) \rho^{8}+\left(\frac{189}{5} \epsilon^{4} \mathcal{Q}^{3} \ln \epsilon+\frac{9729}{400} \epsilon^{4} \mathcal{Q}^{3}-\frac{189}{4} \epsilon^{4} \mathcal{Q}^{3} \ln 2\right. \\
& \left.\left.+\frac{567}{20} \epsilon^{4} \mathcal{Q}^{3} \ln 3+\mathcal{Q}^{9}+\frac{567}{10} \epsilon^{4} \mathcal{Q}^{3} \ln \rho\right) \rho^{9}+\mathcal{O}\left(\rho^{10} \ln \rho\right)\right)
\end{aligned}
$$

where

$$
\begin{aligned}
\mathcal{Q} & =\frac{\sqrt{6}(2 \epsilon)^{2 / 3}}{4}\left\{\int_{0}^{1} d u\left(\frac{1-u^{6}}{u^{2}\left(1-u^{12}+12 u^{6} \ln u\right)^{1 / 3}}-\frac{1}{u^{2}}\right)-1\right\} \\
& =-\frac{\sqrt{6}(2 \epsilon)^{2 / 3}}{4} \times 0.839917(9) .
\end{aligned}
$$

\footnotetext{
${ }^{4}$ In the previous studies of the cascading gauge theory on $S^{3}$ [10] or in $d S_{4}$ [15], the strong coupling scale was defined as $\Lambda_{S^{3}}=\Lambda_{d S_{4}}=2^{-1 / 4} \Lambda$, relative to $\Lambda$ in (2.39).
} 
In the IR, $\tau \rightarrow 0, z \rightarrow 1_{-}$and $y \equiv \frac{1}{\rho} \rightarrow 0$ we have

$$
\tau=\frac{\sqrt{6} 2^{1 / 3}}{3^{1 / 3} \epsilon^{2 / 3}} y\left(1-\frac{2^{2 / 3} 3^{1 / 3}}{15 \epsilon^{4 / 3}} y^{2}+\frac{713^{2 / 3} 2^{1 / 3}}{2625 \epsilon^{8 / 3}} y^{4}+\mathcal{O}\left(y^{6}\right)\right)
$$

Given an analytic Klebanov-Strassler solution (2.31)-(2.33), (2.36)-(2.38), and the asymptotic correspondence between $\tau$ and $\rho$ (or $y$ ) coordinates (2.43) and (2.45) it is straightforward to express all the KS background functions asymptotically as $\tau \rightarrow \infty(\rho \rightarrow 0)$ and $\tau \rightarrow 0(\rho \rightarrow \infty)$. Of particular importance is the UV asymptote of the NS-NS 3 -form flux function $K_{1}$, to be defined below in (3.14), related to $h_{1, K S}$ :

$$
K_{1} \equiv 54 M \alpha^{\prime} h_{1, K S}=\left(\frac{9}{2} M \alpha^{\prime}\right)^{2} g_{s}\left\{\ln \left[\frac{2^{1 / 2}}{\Lambda^{2}}\left(\frac{2}{9 M \alpha^{\prime}}\right)^{2} \frac{1}{g_{s}}\right]-2 \ln \rho+\mathcal{O}\left(\rho^{3} \ln \rho\right)\right\},
$$

where we used (2.43) and the definition of the strong coupling scale $\Lambda$ (2.39). The somewhat grotesque definition of $\Lambda$, leading to (2.46) becomes natural as we construct numerical solutions in sections 5-6, using $P \propto M \alpha^{\prime}$ as in (3.6) and the asymptotic parametrization of $K_{1}$ as in (A.15)

$$
K_{1}=K_{0}-2 P^{2} g_{s} \ln \rho+\mathcal{O}\left(\rho^{3} \ln \rho\right) .
$$

Indeed, we find in this case

$$
\Lambda^{2}=\frac{\sqrt{2}}{P^{2} g_{s}} e^{-\frac{K_{0}}{P^{2} g_{s}}}
$$

where the factor $\sqrt{2}$ is introduced for historical reasons, to agree with the analysis in $[3,4$, $11]$.

\section{Review: type IIB supergravity on warped deformed conifold with fluxes}

Consistent truncation in the $\mathrm{SU}(2) \times \mathrm{SU}(2) \times \mathbb{Z}_{2}$ invariant sector of Type IIB supergravity on warped deformed conifold with fluxes to a five dimensional manifold $\mathcal{M}_{5}$ was derived in [11]:

$$
\begin{gathered}
S_{5}\left[g_{\mu \nu}, \Omega_{i=1 \cdots 3}, \Phi, h_{i=1 \cdots 3},\left\{P, \Omega_{0}\right\}\right]=\frac{108}{16 \pi G_{5}} \int_{\mathcal{M}_{5}} \operatorname{vol}_{\mathcal{M}_{5}} \Omega_{1} \Omega_{2}^{2} \Omega_{3}^{2} \\
\times\left\{R_{10}-\frac{1}{2}(\nabla \Phi)^{2}-\frac{1}{2} e^{-\Phi}\left(\frac{\left(h_{1}-h_{3}\right)^{2}}{2 \Omega_{1}^{2} \Omega_{2}^{2} \Omega_{3}^{2}}+\frac{1}{\Omega_{3}^{4}}\left(\nabla h_{1}\right)^{2}+\frac{1}{\Omega_{2}^{4}}\left(\nabla h_{3}\right)^{2}\right)\right. \\
\quad-\frac{1}{2} e^{\Phi}\left(\frac{2}{\Omega_{2}^{2} \Omega_{3}^{2}}\left(\nabla h_{2}\right)^{2}+\frac{1}{\Omega_{1}^{2} \Omega_{2}^{4}}\left(h_{2}-\frac{P}{9}\right)^{2}+\frac{1}{\Omega_{1}^{2} \Omega_{3}^{4}} h_{2}^{2}\right) \\
\left.-\frac{1}{2 \Omega_{1}^{2} \Omega_{2}^{4} \Omega_{3}^{4}}\left(4 \Omega_{0}+h_{2}\left(h_{3}-h_{1}\right)+\frac{1}{9} P h_{1}\right)^{2}\right\} .
\end{gathered}
$$

It is a functional of:

- a five-dimensional metric $g_{\mu \nu}$ on $\mathcal{M}_{5}$,

$$
d s_{5}^{2}=g_{\mu \nu}(y) d y^{\mu} d y^{\nu},
$$


- three scalars $\Omega_{i=1 \cdots 3}(y)$ uplifting the metric on $\mathcal{M}_{5}$ to a 10 -dimensional metric of Type IIB supergravity,

$$
d s_{10}^{2}=d s_{5}^{2}+d s_{T^{1,1}}^{2}, \quad d s_{T^{1,1}}^{2}=\Omega_{1}^{2}(y) g_{5}^{2}+\Omega_{2}^{2}(y)\left(g_{3}^{2}+g_{4}^{2}\right)+\Omega_{3}^{2}(y)\left(g_{1}^{2}+g_{2}^{2}\right),
$$

- a dilaton $\Phi(y)$,

- three scalars $h_{i=1 \cdots 3}(y)$, uplifting to a R-R 3-form flux $F_{3}=F_{3}^{\text {top }}+d C_{2}$ and an NS-NS 3-form flux $H_{3}=d B_{2}$ as

$$
\begin{aligned}
B_{2} & =h_{1}(y) g_{1} \wedge g_{2}+h_{3}(y) g_{3} \wedge g_{4}, \quad C_{2}=h_{2}(y)\left(g_{1} \wedge g_{3}+g_{2} \wedge g_{4}\right), \\
F_{3}^{\text {top }} & =\frac{1}{9} P g_{5} \wedge g_{3} \wedge g_{4} .
\end{aligned}
$$

The topological part of the R-R 3-from flux $F_{3}^{\text {top }}$ depends on a fixed parameter $P$, subject to quantization $[3,13]$ :

$$
\frac{1}{4 \pi^{2} \alpha^{\prime}} \int_{3-\text { cycle }} F_{3}^{\text {top }}=\frac{2 P}{9 \alpha^{\prime}} \in \mathbb{Z},
$$

leading to

$$
P=\frac{9}{2} M \alpha^{\prime},
$$

where $M$ is the number of fractional D3 branes (the number of D5 branes wrapping the 2-cycle of the conifold base). ${ }^{5}$

The self-dual R-R five-form $F_{5}=\mathcal{F}_{5}+\star \mathcal{F}_{5}$ Bianchi identity

$$
d F_{5}=H_{3} \wedge F_{3}
$$

can be integrated to obtain

$$
g_{s} \mathcal{F}_{5}=\left(4 \Omega_{0}+h_{2}(y)\left(h_{3}(y)-h_{1}(y)\right)+\frac{P}{9} h_{1}(y)\right) g_{1} \wedge g_{2} \wedge g_{3} \wedge g_{4} \wedge g_{5},
$$

where $\Omega_{0}$ is an arbitrary constant. In the absence of the 3 -form fluxes (the vanishing r.h.s. of (3.7)), the 5-form is conserved and must be quantized [13]

$$
\frac{1}{\left(4 \pi^{2} \alpha^{\prime}\right)^{2}} \int_{T^{1,1}} \mathcal{F}_{5}=\frac{16 \Omega_{0}}{\pi g_{s}\left(\alpha^{\prime}\right)^{2}} \in \mathbb{Z}
$$

leading to

$$
\Omega_{0}=\frac{\pi g_{s}\left(\alpha^{\prime}\right)^{2} N}{16}
$$

where $N$ is the number of D3 branes placed at the tip of the singular conifold, and $g_{s}$ is the asymptotic string coupling,

$$
e^{\Phi} \longrightarrow g_{s}
$$

\footnotetext{
${ }^{5}$ The $T^{1,1} 3$-cycle is parameterized by $\theta_{2}=\phi_{2}=0$, and the 2-cycle is parameterized by $\psi=0, \theta_{1}=-\theta_{2}$ and $\phi_{1}=-\phi_{2}$, see [13].
} 
Note that $R_{10}$ in (3.1) is given by

$$
\begin{aligned}
R_{10}= & R_{5}+\left(\frac{1}{2 \Omega_{1}^{2}}+\frac{2}{\Omega_{2}^{2}}+\frac{2}{\Omega_{3}^{2}}-\frac{\Omega_{2}^{2}}{4 \Omega_{1}^{2} \Omega_{3}^{2}}-\frac{\Omega_{3}^{2}}{4 \Omega_{1}^{2} \Omega_{2}^{2}}-\frac{\Omega_{1}^{2}}{\Omega_{2}^{2} \Omega_{3}^{2}}\right)-2 \square \ln \left(\Omega_{1} \Omega_{2}^{2} \Omega_{3}^{2}\right) \\
& -\left\{\left(\nabla \ln \Omega_{1}\right)^{2}+2\left(\nabla \ln \Omega_{2}\right)^{2}+2\left(\nabla \ln \Omega_{3}\right)^{2}+\left(\nabla \ln \left(\Omega_{1} \Omega_{2}^{2} \Omega_{3}^{2}\right)\right)^{2}\right\},
\end{aligned}
$$

and $R_{5}$ is the five-dimensional Ricci scalar of the metric (3.2). Furthermore, all the covariant derivatives $\nabla_{\lambda}$ in the effective action (3.1) are computed with respect to the metric (3.2). Finally, $G_{5}$ is the five dimensional effective gravitational constant

$$
G_{5} \equiv \frac{G_{10}}{\operatorname{vol}_{T^{1,1}}}=\frac{27}{16 \pi^{3}} G_{10}
$$

where $16 \pi G_{10}=(2 \pi)^{7} g_{s}^{2}\left(\alpha^{\prime}\right)^{4}$ is 10 -dimensional gravitational constant of Type IIB supergravity.

Our primary application of the effective action (3.1) is in the context of black holes on the warped deformed conifold, realizing the holographic dual to thermal states of the KS cascading gauge theory on $S^{3}$. For this goal, we find it convenient to introduce

$$
\begin{aligned}
& h_{1}=\frac{1}{P}\left(\frac{K_{1}}{12}-36 \Omega_{0}\right), \quad h_{2}=\frac{P}{18} K_{2}, \quad h_{3}=\frac{1}{P}\left(\frac{K_{3}}{12}-36 \Omega_{0}\right), \\
& \Omega_{1}=\frac{1}{3} f_{c}^{1 / 2} h^{1 / 4}, \quad \Omega_{2}=\frac{1}{\sqrt{6}} f_{a}^{1 / 2} h^{1 / 4}, \quad \Omega_{3}=\frac{1}{\sqrt{6}} f_{b}^{1 / 2} h^{1 / 4}, \quad g=e^{\Phi},
\end{aligned}
$$

and following [25] isolate the holographic coordinate $\rho$ in $\mathcal{M}_{5}$ as:

$$
d s_{5}^{2}=g_{\mu \nu}(y) d y^{\mu} d y^{\nu} \equiv \frac{1}{h^{1 / 2}(x, \rho) \rho^{2}}\left(G_{i j}(x, \rho) d x^{i} d x^{j}\right)+\frac{h^{1 / 2}(x, \rho)}{\rho^{2}} g_{\rho \rho}(x, \rho)(d \rho)^{2} .
$$

The boundary of the space $\mathcal{M}_{5}, \partial \mathcal{M}_{5}$, is taken at $\rho \rightarrow 0$, with

$$
\lim _{\rho \rightarrow 0} G_{i j}(x, \rho) d x^{i} d x^{j}=-(d t)^{2}+\frac{1}{\mu^{2}}\left(d S^{3}\right)^{2}, \quad \lim _{\rho \rightarrow 0} g_{\rho \rho}(x, \rho)=1,
$$

where $\left(d S^{3}\right)^{2}$ is the unit size round metric on $S^{3}$, and $L_{3}=\frac{1}{\mu}$ is its radius. Insisting that the $\mathrm{SO}(4)$ symmetry of the boundary metric (3.16) is the symmetry of the full $\mathcal{M}_{5}$ metric (3.15) implies that $G_{i j}$ and $g_{\rho \rho}$ are the functions of the holographic radial coordinate $\rho$ only. ${ }^{6}$ As emphasized in [10], the parametrization (3.15) is not unique - the diffeomorphisms of the type

$$
\left(\begin{array}{c}
\rho \\
h \\
G_{i j} \\
g_{\rho \rho} \\
f_{a, b, c} \\
K_{1,2,3} \\
\Phi
\end{array}\right) \Longrightarrow\left(\begin{array}{c}
\hat{\rho} \\
\hat{h} \\
\hat{G}_{i j} \\
\hat{g}_{\rho \rho} \\
\hat{f}_{a, b, c} \\
\hat{K}_{1,2,3} \\
\hat{\Phi}
\end{array}\right)=\left(\begin{array}{c}
\rho /(1+\alpha \rho) \\
(1+\alpha \rho)^{4} h \\
G_{i j} \\
g_{\rho \rho} \\
(1+\alpha \rho)^{-2} f_{a, b, c} \\
K_{1,2,3} \\
\Phi
\end{array}\right), \quad \alpha=\text { const }
$$

\footnotetext{
${ }^{6}$ Of course, the same implies for all the scalars in the effective action (3.1).
} 
preserve the general form of the metric. For ${ }^{7} \mu \neq 0$, we can completely fix (3.17), i.e., the parameter $\alpha$ in (3.17), requiring that for a geodesically complete $\mathcal{M}_{5}$ the radial coordinate $\rho$ extends as

$$
\rho \in[0,+\infty)
$$

We choose different parameterizations of the metric (3.15) depending on whether or not it has a regular Schwarzschild horizon in the bulk:

$$
\begin{array}{lll}
\text { (A) [no horizon] : } & G_{i j} d x^{i} d x^{j} \equiv-(d t)^{2}+\frac{f_{1}(\rho)^{2}}{\mu^{2}}\left(d S^{3}\right)^{2}, & g_{\rho \rho}=1 ; \\
\text { (B) [horizon] : } & G_{i j} d x^{i} d x^{j} \equiv-f(\rho)(d t)^{2}+\frac{1}{\mu^{2}}\left(d S^{3}\right)^{2}, & g_{\rho \rho}=f^{-1}(\rho) .
\end{array}
$$

From (3.16), at the $\mathcal{M}_{5}$ boundary,

$$
\lim _{\rho \rightarrow 0} f_{1}(\rho)=1, \quad \text { and } \quad \lim _{\rho \rightarrow 0} f(\rho)=1,
$$

while the presence of the horizon as $\rho \rightarrow \infty$ in case (B) implies that

$$
\lim _{\rho \rightarrow \infty} f(\rho)=0 .
$$

Note that in case $(\mathrm{A})$ - no horizon in $\mathcal{M}_{5}-f_{1}(\rho) \rightarrow 0$ as $\rho \rightarrow \infty$ is one of the possible boundary condition dictated by the geodesic completeness of $\mathcal{M}_{5}$; alternatively, we can require for a 2-cycle of the warped deformed conifold to shrink to zero size, much like in the KS solution [2], with $f_{1}$ nonvanishing in the limit.

Given the radial coordinate as in (3.18) and the metric ansatz (3.19), there is an additional rescaling symmetry of the background geometry that effects the energy scale $\mu$ :

$$
\rho \rightarrow \frac{\rho}{\beta}, \quad t \rightarrow \frac{t}{\beta}, \quad \mu \rightarrow \beta \mu, \quad \beta=\text { const },
$$

with the rest of the background defining functions ((3.14) and $h, f_{1}$ or $\left.f\right)$ unchanged. Clearly, under the symmetry transformation (3.22) any physical observable $\mathcal{O}$ of a massscaling dimension $\Delta$ would transform as

$$
\mathcal{O} \rightarrow \beta^{\Delta} \mathcal{O}
$$

In numerical analysis in sections $5-6$ we fix this symmetry either by fixing $\mu$ or the rescaling of the radial coordinate - the results of the computations will be presented as dimensionless ratios, invariant under (3.22).

Finally, there are two rescaling symmetries acting on $P$ as $P \rightarrow \lambda P$ : with

$$
g \rightarrow \frac{g}{\lambda},\left\{\rho, \mu, G_{i j}, f_{a, b, c}, h, K_{1,2,3}\right\} \rightarrow\left\{\rho, \mu, G_{i j}, f_{a, b, c}, h, K_{1,2,3}\right\},
$$

\footnotetext{
${ }^{7}$ When $\mu \rightarrow 0$, i.e., in the decompactification limit $S^{3} \rightarrow \mathbb{R}^{3}$, there is a residual rescaling of the radial coordinate $\rho \rightarrow \lambda \rho$, with the constant $\lambda$, where $\lambda$ is absorbed into the rescaling of the time $t \rightarrow \lambda t$ and the $\mathbb{R}^{3}$ spatial coordinates $x^{i} \rightarrow \lambda x^{i}$, with all the fields of the effective action (3.1) left invariant.
} 
or with

$$
\rho \rightarrow \frac{\rho}{\lambda},\left\{h, K_{1,3}\right\} \rightarrow \lambda^{2}\left\{h, K_{1,3}\right\},\left\{\mu, G_{i j}, f_{a, b, c}, K_{2}, g\right\} \rightarrow\left\{\mu, G_{i j}, f_{a, b, c}, K_{2}, g\right\} .
$$

The former symmetry can be used to set the asymptotic string coupling as

$$
\lim _{\rho \rightarrow 0} g=1 \equiv g_{s}
$$

while the latter can be used to either set $P=1$, or to set the asymptotic parameter $K_{0}$, see (2.47), since under (3.25)

$$
\frac{K_{0}}{P^{2} g_{s}} \rightarrow \frac{K_{0}}{P^{2} g_{s}}-2 \ln \lambda
$$

Generic solutions of the effective action (3.1) have only $\mathbb{Z}_{2} R$-symmetry; this chiral symmetry is enhanced to $\mathrm{U}(1)$ in solutions of the consistent truncation of the latter action [25], constraining

$$
K_{1}=K_{3}, \quad f_{a}=f_{b}, \quad K_{2} \equiv 1 .
$$

In appendix A we collect equations of motion derived from the effective action (3.1), produce the corresponding asymptotic expansions, and review the holographic renormalization of the model. Additionally, in appendix A.1.1, we recast the supersymmetric solution of the Klebanov-Strassler [2], reviewed in section 2, as the spatial $S^{3}$-decompactification limit, i.e., $\mu \rightarrow 0$, of the warped deformed conifold vacua $\mathcal{V}_{B}$. We collect explicit expressions for the boundary stress-energy tensor in vacua and in thermal states of the model in appendix A.4.

\section{Vacua and black holes in $A d S_{5} \times T^{1,1}$}

Prior to moving to a more technical example of Type IIB flux geometries on warped deformed conifold, we consider the vacua and the black holes on a singular conifold, with the self-dual flux $F_{5}$ only. For large values of the flux these solutions represent the vacua and the thermal states of large- $N$ Klebanov-Witten theory [12] on $\mathcal{M}_{4}=R \times S^{3}$. There are not new results here: we just present this textbook example in the framework of the effective action 3 .

Note that the absence of the three form fluxes implies (see (3.14))

$$
P=0, \quad K_{1}=K_{3} \equiv K_{0}=432 \Omega_{0}, \quad K_{2} \equiv 1,
$$

where $\Omega_{0}$ is quantized as in (3.10).

\subsection{Conformal $\mathcal{V}_{A}^{s}$ vacua}

The equations of motion for horizonless bulk geometries of Type IIB supergravity (3.1), dual to Klebanov-Witten gauge theory vacua on $S^{3}$ are collected in appendix A.1. The limit $P \rightarrow 0$ of these equations is slightly subtle: first we need to set $K_{i}$ as in (4.1), followed by setting $P=0$. We find: 
- from (A.9):

$$
g(\rho) \equiv g_{s}
$$

- from (A.7):

$$
f_{b}(\rho)=f_{a}(\rho)
$$

- Equations (A.2) and (A.3) become identities once we set

$$
f_{c}(\rho)=f_{a}(\rho) .
$$

- We expect that the warp factors $\Omega_{i}$ in (3.14) are constant, thus we set

$$
f_{a}(\rho)=\frac{Q}{\sqrt{h(\rho)}} .
$$

Consistency of (A.2), (A.5) and (A.10) implies that

$$
Q=\frac{1}{2} K_{0}^{1 / 2}
$$

Notice that the "size" of the compact space $T^{1,1}$ is (see (4.1) and (3.10))

$$
L^{4} \equiv Q^{2}=\frac{K_{0}}{4}=108 \Omega_{0}=\frac{27}{4} \pi g_{s} N\left(\alpha^{\prime}\right)^{2},
$$

in agreement with (2.25). In what follows, we present the solution in terms of $K_{0}$, rather than $L$ from (4.7).

- We are left at this stage with the 3 equations, i.e., (A.1), (A.5) and (A.10), for the remaining functions $f_{1}, h$ and the first-order constraint. The most efficient way to proceed is to introduce

$$
\tilde{h} \equiv \frac{h}{f_{1}^{4}} .
$$

The new function must be defined on the interval (3.18), and from (A.14) and (A.19), and (A.23), must satisfy the boundary conditions:

$$
\lim _{\rho \rightarrow 0} \tilde{h}=\frac{K_{0}}{4}, \quad \lim _{\rho \rightarrow \infty} \tilde{h}=\frac{1}{\mu^{4} h_{0}^{h}}=\text { finite }
$$

Eliminating $f_{1}^{\prime}$ from (A.10), we find a decoupled equation for $\tilde{h}$ :

$$
0=\tilde{h}^{\prime \prime}-\frac{5\left(\tilde{h}^{\prime}\right)^{2}}{4 \tilde{h}}
$$

The most general solution is

$$
\tilde{h}=\frac{\mathcal{H}_{1}}{\left(1+\mathcal{H}_{2} \rho\right)^{4}},
$$

where $\mathcal{H}_{i}$ are the integration constants. Imposing the boundary conditions (4.9) we determine

$$
\tilde{h} \equiv \frac{K_{0}}{4}
$$


- Given (4.12), we solve (A.1), subject to the boundary condition (3.20),

$$
f_{1}=\frac{2}{\left(4+K_{0} \mu^{2} \rho^{2}\right)^{1 / 2}} .
$$

From (4.8) we find

$$
h=\frac{4 K_{0}}{\left(4+K_{0} \mu^{2} \rho^{2}\right)^{2}} .
$$

Equipped with the analytic solution, we can extract the UV parameters (A.21):

$$
f_{a, 1,0}=f_{a, 3,0}=k_{2,3,0}=f_{a, 4,0}=f_{c, 4,0}=g_{4,0}=f_{a, 6,0}=k_{2,7,0}=f_{a, 8,0}=0,
$$

and the IR parameters (A.24):

$$
\begin{aligned}
f_{a, 0}^{h} & =f_{b, 0}^{h}=f_{c, 0}^{h}=\frac{\mu^{2} K_{0}}{4}, & h_{0}^{h} & =\frac{4}{\mu^{4} K_{0}}, \\
K_{1,0}^{h} & =K_{3,0}^{h}=K_{0}, & K_{2,0}^{h} & =1, \quad g_{0}^{h}=g_{s} .
\end{aligned}
$$

Using (A.83) in the limit $P \rightarrow 0$ we find for the energy density $\mathcal{E}_{0}$ and the pressure $\mathcal{P}$

$$
\mathcal{E}_{0}=3 \mathcal{P}=\frac{1}{8 \pi G_{5}} \frac{K_{0}^{2} \mu^{4}}{32}=\frac{c}{2 \pi^{2}} \mu^{4},
$$

where in the last equality we used the expression for $\Omega_{0}$ from (3.10), $G_{5}$ from (3.13), and the expression for the central charge $c$ of the large- $N$ Klebanov-Witten gauge theory (2.18), resulting in the Casimir energy

$$
E_{0}=\mathcal{E}_{0} \times \frac{1}{\mu^{3}} \operatorname{vol}\left(S^{3}\right)=c \mu .
$$

Note that (4.18) obtained using the holographic renormalization of [25] is different from the standard result for the Casimir energy of the holographic CFT [26]:

$$
E_{0}^{b=0}=\frac{3 c}{4} \mu .
$$

We put the superscript $b=0$ in (4.19) to emphasize that this result was obtained in the holographic renormalization scheme where the ambiguous coefficient $b$ multiplying $\square R$ of the conformal theory boundary stress-energy tensor trace vanishes, see (2.6). As detailed in [25], the minimal holographic renormalization of the flux geometries, reviewed in section A.3, (dual to the cascading gauge theory on generic $\mathcal{M}_{4}$ ) requires the presence of $\mathcal{R}_{\gamma}^{2}$ and $\mathcal{R}_{a b \gamma} \mathcal{R}_{\gamma}^{a b}$ counterterms (see (A.68)). These counterterms, in the conformal, i.e., $P \rightarrow 0$, limit produce the $\square R$ term in the boundary stress-energy tensor (see eq. (3.54) in [25]) as

$$
+\frac{1}{8 \pi G_{5}} \square R \times \frac{1}{384} K_{0}^{2}=+\frac{c}{24 \pi^{2}} \square R \equiv \frac{1}{(4 \pi)^{2}} \times \underbrace{\frac{2 c}{3}}_{\equiv b} \times \square R,
$$

which comparing with (2.6) implies that $b=\frac{2 c}{3}$. Thus, following (2.9), we expect that

$$
E_{0}=E_{0}^{b=0}+\delta E_{0}^{b=2 c / 3}=\frac{3 c}{4} \mu+\frac{3}{8} \times \frac{2 c}{3} \mu=c \mu,
$$

which is indeed the case. 


\subsection{Conformal $\mathcal{T}_{\text {decon }}^{s}$ thermal states}

The equations of motion for black hole geometries of Type IIB supergravity (3.1), dual to Klebanov-Witten gauge theory deconfined thermal states on $S^{3}$ are collected in appendix A.2. To solve these equations we follow the same steps as in section 4.1. We find

$$
\begin{aligned}
& f=\frac{4\left(f_{a, 1,0} \rho+1\right)\left(K_{0} \mu^{2} \rho^{2}+2 f_{a, 1,0}^{2} \rho^{2}+4 f_{a, 1,0} \rho+4\right)}{\left(f_{a, 1,0} \rho+2\right)^{4}}, \quad f_{a}=f_{b}=f_{c}=\frac{\left(f_{a, 1,0} \rho+2\right)^{2}}{4}, \\
& h=\frac{4 K_{0}}{\left(f_{a, 1,0} \rho+2\right)^{4}}, \quad K_{1}=K_{3} \equiv K_{0}, \quad K_{2} \equiv 1, \quad g \equiv g_{s},
\end{aligned}
$$

resulting in the UV parameters (A.55)

$$
\begin{aligned}
f_{4,0} & =\frac{1}{16} f_{a, 1,0}^{2}\left(2 K_{0} \mu^{2}-f_{a, 1,0}^{2}\right), \\
f_{a, 3,0} & =k_{2,3,0}=f_{c, 4,0}=g_{4,0}=f_{a, 6,0}=k_{2,7,0}=f_{c, 8,0}=0,
\end{aligned}
$$

and the IR parameters (A.58)

$$
\begin{aligned}
f_{a, 0}^{h} & =f_{b, 0}^{h}=f_{c, 0}^{h}=\frac{1}{4} f_{a, 1,0}^{2}, & h_{0}^{h} & =\frac{4 K_{0}}{f_{a, 1,0}^{4}}, \quad K_{1,0}^{h}=K_{3,0}^{h}=K_{0}, \quad K_{2,0}^{h}=1, \\
g_{0}^{h} & =g_{s}, & f_{1}^{h} & =\frac{4\left(K_{0} \mu^{2}+2 f_{a, 1,0}^{2}\right)}{f_{a, 1,0}^{3}} .
\end{aligned}
$$

While $K_{0}$ is a fixed parameter, specifying the size of $T^{1,1}$, see (4.7), the parameter $f_{a, 1,0}$, which we express as

$$
f_{a, 1,0} \equiv K_{0}^{1 / 2} \kappa,
$$

is not fixed, and instead encodes the temperature of the state (A.59):

$$
T=\frac{\mu^{2}+2 \kappa^{2}}{2 \pi \kappa} .
$$

From section A.4.4, the remaining thermodynamic characteristics of the deconfined state are

$$
\mathcal{E}=3 \mathcal{P}=\frac{c}{2 \pi^{2}}\left(\mu^{4}+3 \kappa^{2}\left(\kappa^{2}+\mu^{2}\right)\right), \quad s=\frac{2 c}{\pi} \kappa^{3}, \quad \mathcal{F}=\frac{c}{2 \pi^{2}}\left(\mu^{4}+\kappa^{2}\left(\mu^{2}-\kappa^{2}\right)\right) .
$$

We end this section summarizing the well-known properties and the phase structure of the black holes in $A d S_{5} \times T^{1,1}$ : the first law of thermodynamics is satisfied:

$$
d \mathcal{E}=\frac{3 c}{\pi^{2}} \kappa\left(2 \kappa^{2}+\mu^{2}\right) \equiv T d s .
$$

The specific heat density $c_{V}$ of small black holes, i.e., the low entropy, is negative:

$$
c_{V}=-T \frac{\partial^{2} \mathcal{F}}{\partial T^{2}}=\frac{6 c \kappa^{3}}{\pi} \frac{2 \kappa^{2}+\mu^{2}}{2 \kappa^{2}-\mu^{2}}<0 \quad \Longleftrightarrow \quad \kappa<\frac{1}{\sqrt{2}} \mu .
$$

The black holes discussed realize the deconfined thermal phase of the Klebanov-Witten gauge theory. The confined thermal phase is represented by the $\mathcal{V}_{A}^{s}$ vacua solutions of 


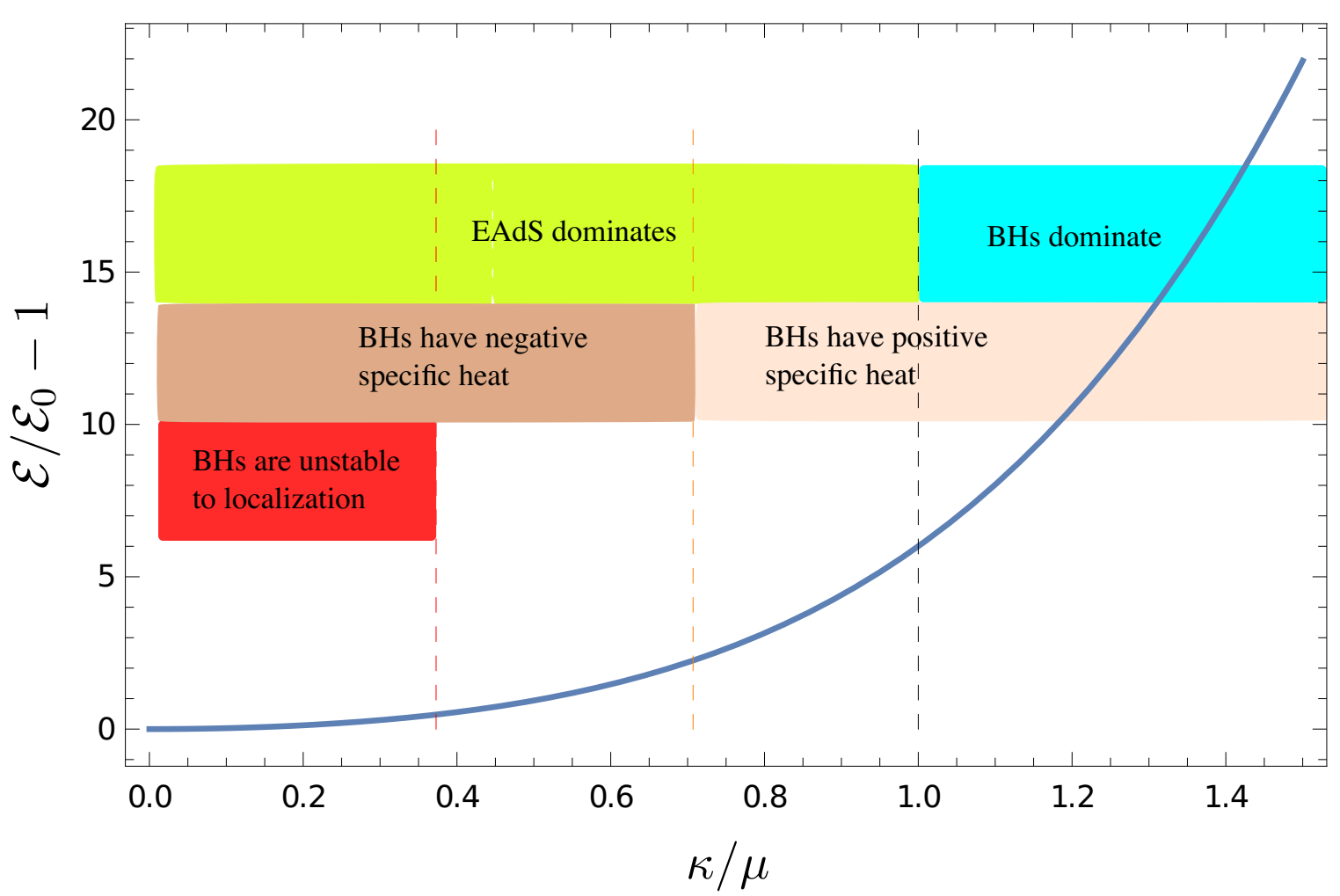

Figure 4. The phase diagram of black holes in $A d S_{5} \times T^{1,1}$. The energy density $\mathcal{E}$ relative to the Casimir energy density $\mathcal{E}_{0}$ is shown as a function of $\kappa / \mu$. $\kappa$ determines the Hawking temperature of the black hole, see (4.26).

section 4.1 with the Euclidean time direction compactified with the appropriate inverse temperature period. The Hawking-Page [8] or the confinement phase transition [7] occurs when (compare (4.27) and (4.17))

$$
\left.\mathcal{F}\right|_{\text {deconfined }} \geq \mathcal{F}=\left.\mathcal{E}\right|_{\text {confined }} \quad \Longleftrightarrow \quad \kappa^{2}\left(\mu^{2}-\kappa^{2}\right) \geq 0
$$

leading to a critical temperature $T_{\text {con/decon }}$ of the confinement/deconfinement phase transition

$$
T_{\text {con } / \text { decon }}=\frac{3 \mu}{2 \pi} .
$$

The black holes considered are 'smeared' over the compact space $-T^{1,1}$ in this case. When the black hole becomes sufficiently small, it becomes unstable with respect to localization on the compact space [27, 28]. From the dual boundary gauge theory perspective, the global $\mathrm{SU}(2) \times \mathrm{SU}(2) \times \mathrm{U}(1)$ symmetry of the Klebanov-Witten gauge theory is spontaneously broken. It was determined in [29] that the $A d S_{5} \times T^{1,1}$ black holes develop such an instability when

$$
\kappa<0.37285(8) \mu \text {. }
$$

In figure 4 we collect the features of the black holes in $A d S_{5} \times S^{5}$. We plot the energy density $\mathcal{E}$ of the black holes relative to their Casimir energy density $\mathcal{E}_{0}(4.17)$ as a 


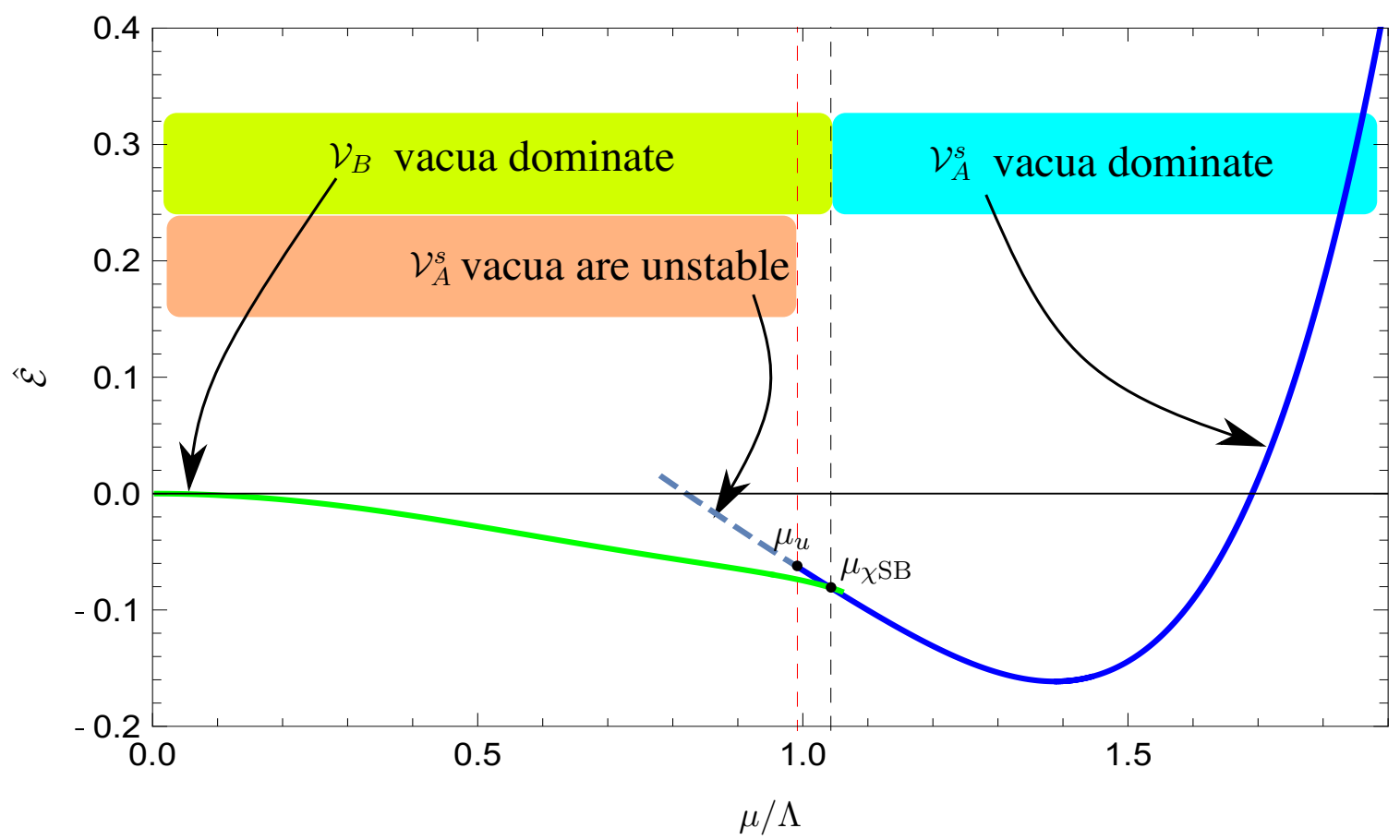

Figure 5. Round $S^{3}$ vacua of the warped deformed conifold with fluxes. The vertical dashed black line indicates the first-order transition between the chirally symmetric $\mathcal{V}_{A}^{s}$ (solid blue curve) and $\mathcal{V}_{B}$ (solid green curve) vacua with the spontaneously broken symmetry, see (5.3). The vertical dashed red line indicates the onset of the perturbative chiral symmetry breaking instability of the $\mathcal{V}_{A}^{s}$ vacua (which are denoted with the grey dashed curve), see (5.4).

function of $\kappa / \mu . \kappa$ is an auxiliary mass scale, which can be traded for the temperature $T$ following (4.26), and $\mu$ is the boundary $S^{3}$ compactification scale. We choose $\kappa$ so that the Hawking-Page (or confinement/deconfinement phase transition from the dual boundary perspective) is at

$$
\left.\frac{\kappa}{\mu}\right|_{\text {Hawking-Page }}=1
$$

Black holes dominate in the canonical ensemble for $\kappa>\mu$, and undergo the first-order phase transition at $\kappa=\mu$. In the microcanonical ensemble, black holes remain thermodynamically stable for $\kappa>\mu / \sqrt{2}$, and develop a perturbative instability as indicate in (4.32).

\section{Round $S^{3}$ vacua of the warped deformed conifold with fluxes}

We begin this section summarizing the results for the $S^{3}$ vacua structure of the warped deformed conifold with fluxes. These vacua realize the holographic dual to the SO(4) invariant ground states of the cascading gauge theory [2] on $S^{3}$ of radius $\frac{1}{\mu}$. The theory has a strong coupling scale $\Lambda$ (2.39). In figures 5 and 6 we present the reduced Casimir energy density $\hat{\mathcal{E}}$, defined as

$$
\hat{\mathcal{E}} \equiv \frac{8 \pi G_{5}}{P^{4} g_{s}^{2}} \frac{\mathcal{E}}{\Lambda^{4}}=\frac{2^{6} \pi^{4}}{3^{5} M^{4}} \frac{\mathcal{E}}{\Lambda^{4}}
$$


where we used (3.13) and (3.6), as a function of $\frac{\mu}{\Lambda}$. Recall that there are 3 distinct types of vacua: $\mathcal{V}_{A}^{s}, \mathcal{V}_{B}$ and $\mathcal{V}_{A}^{b}$, see figure 2: the $\mathcal{V}_{A}^{s}$ vacua realize ground states of the cascading gauge theory with unbroken chiral symmetry; in the bulk gravitational dual they are characterized topologically by the presence of the trivial 3-cycle (the boundary $S^{3}$ can smoothly shrink to a zero size). In the limit $\mu \gg \Lambda$ they resemble the ground states of the conformal Klebanov-Witten gauge theory [12], see section 4.1. These vacua were originally constructed and analyzed in [10].

The $\mathcal{V}_{B}$ vacua realize ground states of the cascading gauge theory with the spontaneously broken chiral symmetry; in the bulk gravitational dual they are characterized topologically by the presence of the trivial 2-cycle (the vanishing 2-cycle of the deformed conifold, see (2.34)). In the limit $\mu / \Lambda \rightarrow 0$ they represent the ground state of the KlebanovStrassler gauge theory on $\mathbb{R}^{3}$. Note that, see (A.85),

$$
\left.\hat{\mathcal{E}}\right|_{\mathrm{KS} \text { on } \mathbb{R}^{3}}=0
$$

These vacua were originally constructed and analyzed in [10].

The $\mathcal{V}_{A}^{b}$ vacua realize ground states of the cascading gauge theory with the spontaneously broken chiral symmetry; in the bulk gravitational dual they are characterized topologically by the presence of the trivial 3-cycle (the boundary $S^{3}$ can smoothly shrink to a zero size). These vacua are constructed and analyzed here for the first time.

In figure 5 the dashed vertical black line denotes the first-order (zero temperature) transition between $\mathcal{V}_{A}^{s}$ (solid blue curve) and $\mathcal{V}_{B}$ (solid green curve) vacua:

$$
\frac{\mu_{\chi \mathrm{SB}}}{\Lambda}=1.043069(7)
$$

This is in perfect agreement with the corresponding transition point obtained in [10]. ${ }^{8} \mathcal{V}_{A}^{s}$ vacua dominate for $\mu>\mu_{\chi \mathrm{SB}}$, implying that the ground state of the cascading gauge theory on sufficiently small $S^{3}$ has unbroken chiral symmetry; while the $\mathcal{V}_{B}$ vacua dominate for $\mu<$ $\mu_{\chi \mathrm{SB}}$, implying that the ground state of the cascading gauge theory on $S^{3}$ spontaneously breaks the chiral symmetry on sufficiently large $S^{3}$. Chirally symmetric $\mathcal{V}_{A}^{s}$ states can be constructed for $\mu<\mu_{\chi \mathrm{SB}}$, however, they become perturbatively unstable for $\mu<\mu_{u}$,

$$
\frac{\mu_{u}}{\Lambda}=0.991613(4)
$$

represented by the dashed vertical red line in figure 5. We represent $\mathcal{V}_{A}^{s}$ vacua for $\mu<\mu_{u}$ with a dashed grey curve. In [10] the instability (5.4) was identified ${ }^{9}$ analyzing the spectrum of the chiral symmetry breaking fluctuations of the theory. Here we identify the instability studying the linearized chiral symmetry breaking fluctuations about $\mathcal{V}_{A}^{s}$ vacua with an explicit symmetry breaking source term [15]. This latter approach would allow us to construct $\mathcal{V}_{A}^{b}$ vacua, which escaped the analysis in [10].

\footnotetext{
${ }^{8}$ The critical value of $\mu$ reported there, see eq. (5.76) in [10], is $2^{1 / 4}$ times bigger corresponding to a $\sqrt{2}$ difference in the definition of the strong coupling scale $\Lambda^{2}(2.48)$ used in this work and in [10].

${ }^{9}$ Again, up to the redefinition of the strong coupling scale $\Lambda$ (2.48), we find a perfect agreement with the earlier computations.
} 


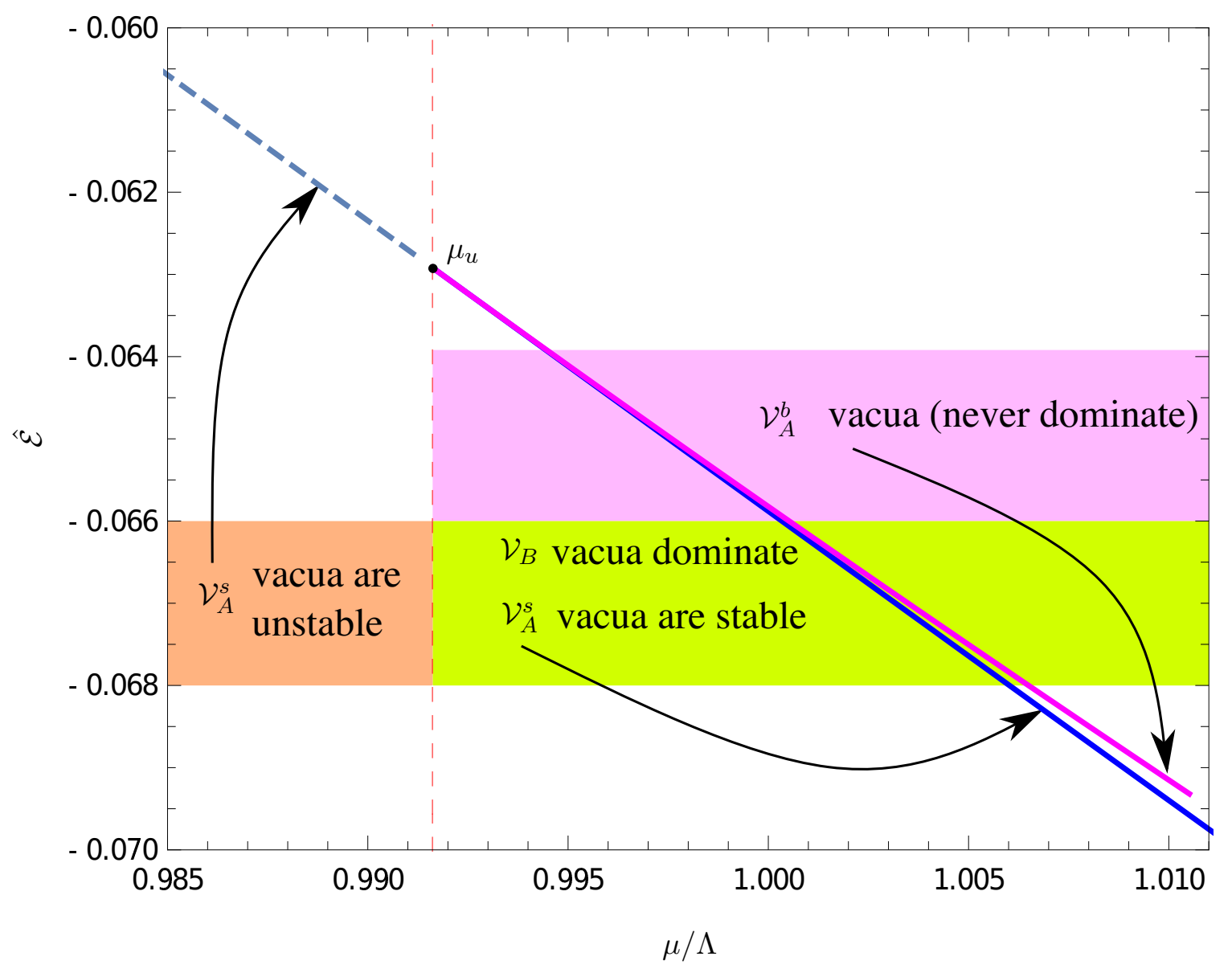

Figure 6. The new branch of $\mathcal{V}_{A}^{b}$ vacua (solid magenta curve) extends for $\mu>\mu_{u}$, see (5.4), represented by the vertical dashed red line.

In figure 6 the solid magenta curve represent $\mathcal{V}_{A}^{b}$ vacua - much like $\mathcal{V}_{B}$, vacua, the dual states of the cascading gauge theory have $\mathbb{Z}_{2}$ chiral symmetry. Since their energy density is above the energy density of $\mathcal{V}_{B}$ states, they are not the true ground states. The scale of the figure does not allow to show this, but (compare with figure 5) for the range of $\mu / \Lambda$ reported,

$$
\left.\hat{\mathcal{E}}\right|_{\mathcal{V}_{A}^{s}}>\left.\hat{\mathcal{E}}\right|_{\mathcal{V}_{B}}
$$

What is exotic in the diagram 6 is that $\mathcal{V}_{A}^{b}$ vacua exist only for $\mu>\mu_{u}$, see (5.4), represented by a vertical dashed red line, where $\mathcal{V}_{A}^{s}$ vacua are perturbatively stable and

$$
\left.\hat{\mathcal{E}}\right|_{\mathcal{V}_{A}^{b}}>\left.\hat{\mathcal{E}}\right|_{\mathcal{V}_{A}^{s}}
$$

i.e., the states with the spontaneously broken symmetry exist above (rather than below!) the energy scale set by the transition point. ${ }^{10}$ We report on the perturbative stability of $\mathcal{V}_{A}^{b}$ states elsewhere. We comment on the fate of the unstable $\mathcal{V}_{A}^{s}$ vacua in section 6 .

\footnotetext{
${ }^{10}$ This is not the first encounter of such a phenomenon: see [30] for the discovery and additional exam-
} 

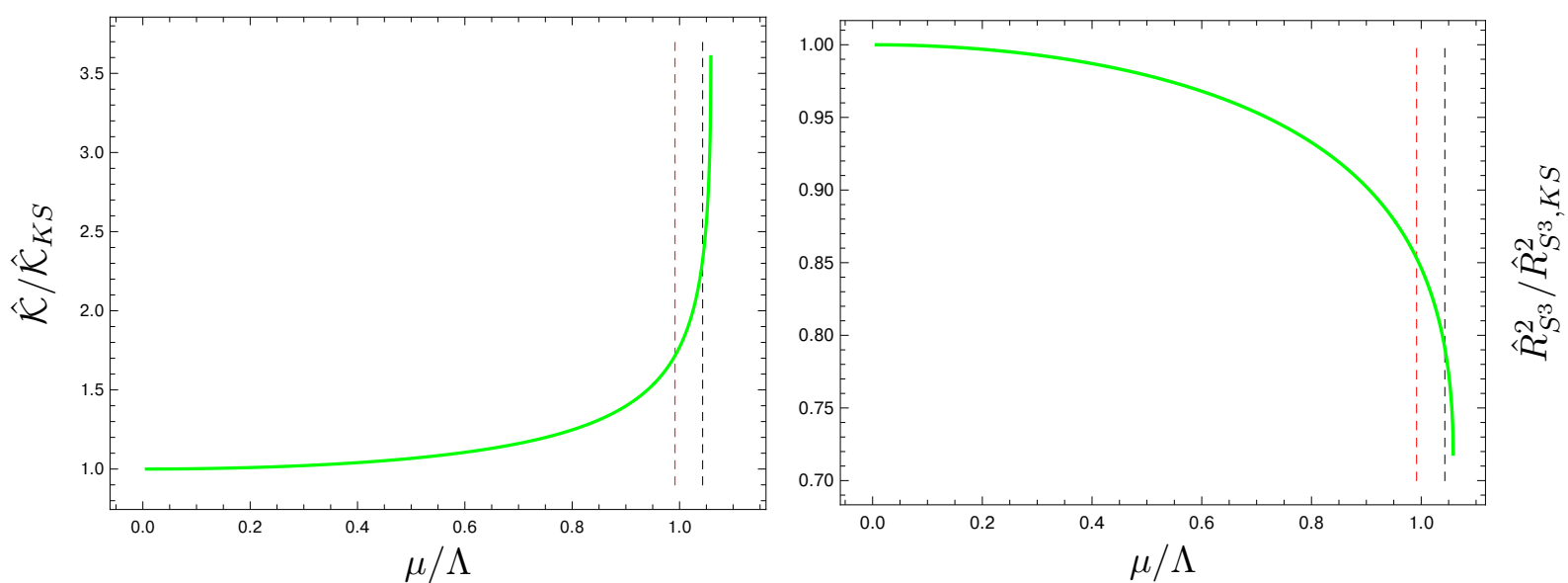

Figure 7. Left panel: the reduced Kretschmann scalar $\hat{\mathcal{K}}$ of $\mathcal{V}_{B}$ vacua in the deep IR of the bulk geometry, see (5.7). $\hat{\mathcal{K}}_{K S}$ denotes the value of the scalar for the Klebanov-Strassler solution, i.e., when $\mu / \Lambda=0$, see (5.8). Right panel: the rapid growth of the scalar when $\mu \gtrsim \mu_{\chi \mathrm{SB}}$ (denoted by the vertical dashed black line) is caused by the collapsing 3-cycle of the conifold, see (5.9). $\hat{R}_{S^{3}, K S}^{2}$ denotes the size of this 3-cycle for the Klebanov-Strassler solution, i.e., when $\mu / \Lambda=0$, see (5.10).
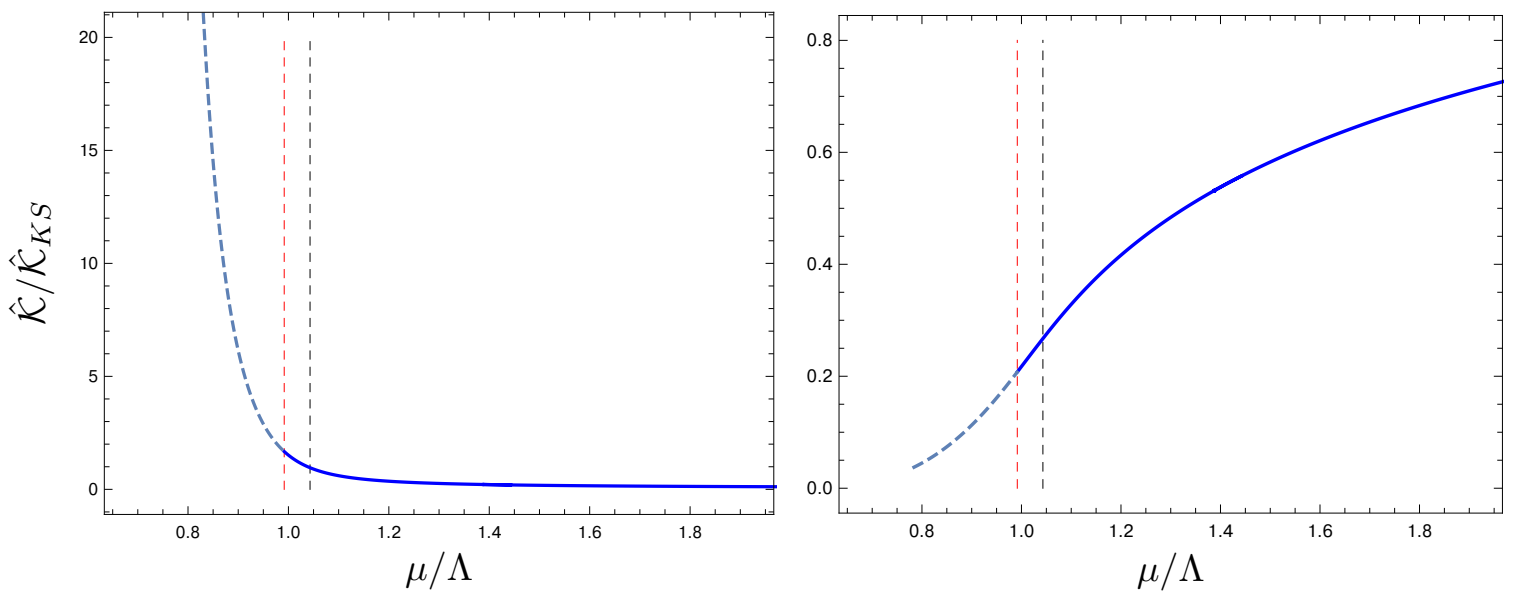

Figure 8. Left panel: the reduced Kretschmann scalar $\hat{\mathcal{K}}$ of $\mathcal{V}_{A}^{s}$ vacua in the deep IR of the bulk geometry, see (5.7). $\hat{\mathcal{K}}_{K S}$ denotes the value of the scalar for the Klebanov-Strassler solution, i.e., when $\mu / \Lambda=0$, see (5.8). Right panel: the rapid growth of the scalar when $\mu \lesssim \mu_{u}$ (denoted by the vertical dashed red line) is caused by the collapsing of the deformed $T^{1,1}$, see (5.11) and (5.12).

We encountered a (technical) obstruction of constructing $\mathcal{V}_{B}$ and $\mathcal{V}_{A}^{b}$ vacua for large values of $\mu$, and $\mathcal{V}_{A}^{s}$ vacua for small values of $\mu$. The physical reason for the obstruction is easy to understand focusing on the invariant properties of the corresponding geometries. In the left panels of figures $7-9$ we plot the reduced Kretschmann scalar $\hat{\mathcal{K}}$, defined as

$$
\hat{\mathcal{K}} \equiv P^{2} g_{s} \times\left.\mathcal{R}_{\mu \nu \lambda \rho} \mathcal{R}^{\mu \nu \lambda \rho}\right|_{I R},
$$

where the ten-dimensional bulk Riemann tensor quadratic invariant is evaluated in the ples $[4,31-35]$ in holography and [36] in QFTs. 

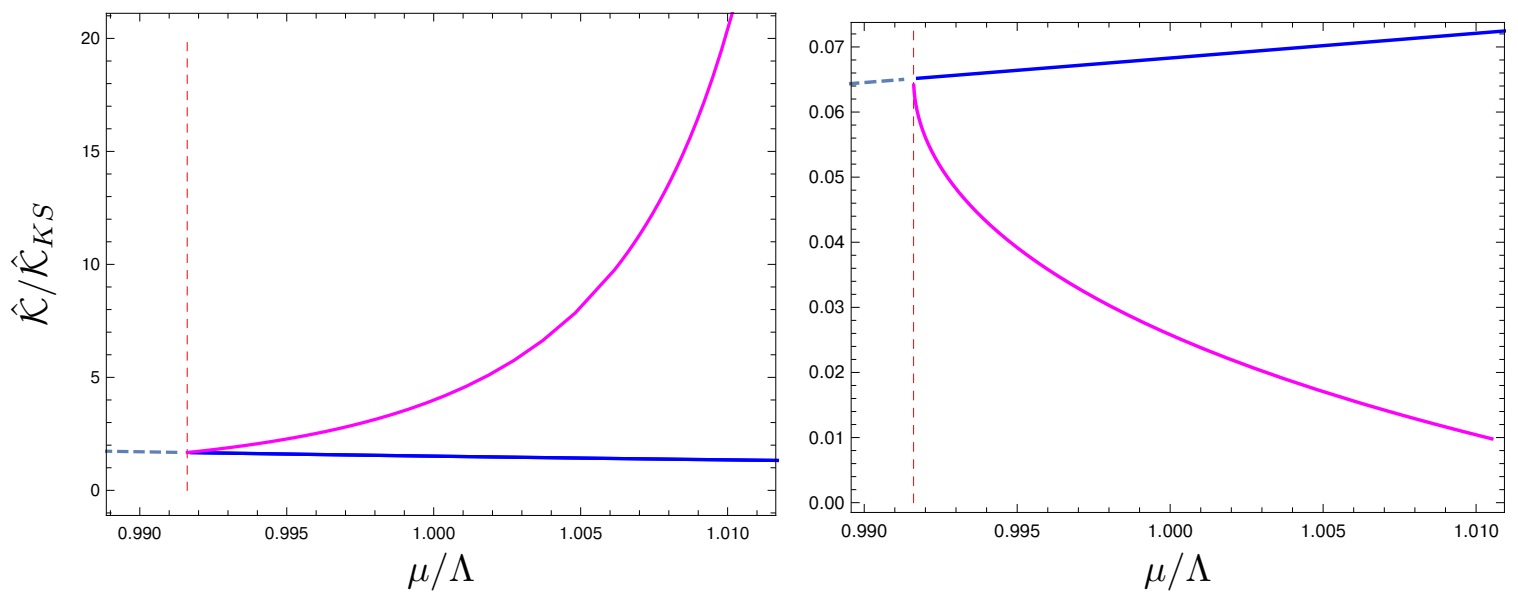

Figure 9. Left panel: the reduced Kretschmann scalar $\hat{\mathcal{K}}$ of $\mathcal{V}_{A}^{b}$ vacua (magenta curve) and $\mathcal{V}_{A}^{s}$ vacua (blue/grey curves) in the deep IR of the bulk geometry, see (5.7). $\hat{\mathcal{K}}_{K S}$ denotes the value of the scalar for the Klebanov-Strassler solution, i.e., when $\mu / \Lambda=0$, see (5.8). Right panel: the rapid growth of the scalar (magenta curve, $\mathcal{V}_{A}^{b}$ vacua) when $\mu \gtrsim \mu_{u}$ (denoted by the vertical dashed red line) is caused by the collapsing 2-cycle of the conifold, see (5.13).

deep IR of the geometry, i.e., as $y \equiv \frac{1}{\rho} \rightarrow 0$, as a function of $\mu / \Lambda$. The quantity

$$
\hat{\mathcal{K}}_{K S}=\frac{64 \times 3^{2 / 3} 2^{1 / 3} \times\left(110 \times 3^{1 / 3} 2^{2 / 3}+177147 H_{0}^{2}\right)}{295245 H_{0}^{3}}
$$

represents the reduced Kretschmann scalar of the Klebanov-Strassler geometry, i.e., when $\mu / \Lambda \rightarrow 0 . \quad H_{0} \equiv 0.056288(0)$ is the numerical coefficient in the IR asymptote of $h_{0}^{h}$, see (A.34). Interestingly, $\hat{\mathcal{K}}_{K S}$, as defined, is independent of the strong coupling scale $\Lambda$ of the cascading gauge theory, equivalently the conifold deformation parameter $\epsilon$, see (2.39). Clearly, the obstruction in extending the construction of the flux vacua is associated with the rapid growth of the quadratic curvature invariant in the deep IR of the geometry, leading to the breakdown of the supergravity approximation. This is associated with an additional cycle ${ }^{11}$ becoming small in the IR. As demonstrated in the right panels of figures $7-9$, what is precisely this additional cycle, differs: from the right panel in figure 7 for $\mathcal{V}_{B}$ vacua, this is a conifold 3-cycle (A.32). We define the reduced radius square of the cycle (again evaluated in the IR, $y \rightarrow 0)$ as

$$
\hat{R}_{S^{3}}^{2} \equiv\left(P^{2} g_{s}\right)^{-1 / 2} \times \frac{f_{a, 0}^{h}\left(h_{0}^{h}\right)^{1 / 2}}{3} .
$$

Note that the corresponding 3-cycle of the Klebanov-Strassler solution has a reduced size (again independent of $\Lambda$ or $\epsilon$ )

$$
\hat{R}_{S^{3}, K S}^{2} \equiv\left(P^{2} g_{s}\right)^{-1 / 2} \times\left(\frac{2}{3}\right)^{1 / 3} H_{0}^{1 / 2}
$$

\footnotetext{
${ }^{11}$ This is an additional cycle to the already collapsed one: the boundary $S^{3}$ for $\mathcal{V}_{A}^{s}$ vacua, the conifold 2-cycle for $\mathcal{V}_{B}$ vacua, and the boundary $S^{3}$ for $\mathcal{V}_{A}^{b}$ vacua - see figure 2.
} 
From the right panel in figure 8 for $\mathcal{V}_{A}^{s}$ vacua, this is (deformed) $T^{1,1}$ (compare with (2.23)):

$$
\left.\left(d \tilde{T}^{1,1}\right)^{2}\right|_{I R}=f_{a, 0}^{h}\left(h_{0}^{h}\right)^{1 / 2}\left(\frac{f_{c, 0}^{h}}{f_{a, 0}^{h}} \frac{1}{9} g_{5}^{2}+\frac{1}{6}\left(g_{3}^{2}+g_{4}^{2}\right)+\frac{1}{6}\left(g_{1}^{2}+g_{2}^{2}\right)\right) .
$$

We define the reduced radius square of the cycle (from (5.11)) as

$$
\hat{R}_{T^{1,1}}^{2} \equiv\left(P^{2} g_{s}\right)^{-1 / 2} \times\left(f_{c, 0}^{h}\right)^{1 / 5}\left(f_{a, 0}^{h}\right)^{4 / 5}\left(h_{0}^{h}\right)^{1 / 2} .
$$

From the right panel in figure 9 for $\mathcal{V}_{A}^{b}$ vacua, this is a conifold 2-cycle (A.31). We define the reduced radius square of the cycle (again evaluated in the IR, $y \rightarrow 0$ ) as

$$
\hat{R}_{S^{2}}^{2} \equiv\left(P^{2} g_{s}\right)^{-1 / 2} \times \frac{f_{b, 0}^{h}\left(h_{0}^{h}\right)^{1 / 2}}{3}
$$

As before, the vertical dashed black line indicates (5.3), and the vertical dashed red line indicates (5.4).

In the rest of this section we explain the technical details leading to the results reported above.

\section{$5.1 \mathcal{V}_{B}$ vacua}

These vacua were originally constructed in [10]. Equations of motion describing $\mathcal{V}_{B}$ vacua are collected in (A.1)-(A.10), with the UV, i.e., $\rho \rightarrow 0_{+}$, asymptotics (A.11)-(A.16), and the IR, i.e., $y \equiv \frac{1}{\rho} \rightarrow 0_{+}$, asymptotics (A.27), (A.28). There are 4 non-normalizable coefficients

$$
K_{0}, \mu, P, g_{s},
$$

characterizing the cascading gauge theory (A.20), and 17 normalizable coefficients (A.21) and (A.29) - precisely as needed to solve the system of 8 second-order equations (A.1)(A.3), (A.5)-(A.9) and the first-order constraint (A.10).

The numerical techniques for solving the required system of nonlinear ODEs were developed in [3]. The scaling symmetries (3.24) and (3.25) are used to set

$$
g_{s}=1 \quad \text { and } \quad P=1 .
$$

Additionally, note that (3.22) acts on $K_{0}$ as

$$
\frac{K_{0}}{P^{2} g_{s}} \rightarrow \frac{K_{0}}{P^{2} g_{s}}-2 \ln \beta
$$

which can be used to keep

$$
\frac{K_{0}}{P^{2} g_{s}}=\text { constant } \equiv k_{s} .
$$

It is important to keep in mind that the symmetry transformation (3.22) affects all the dimensionful observables, and so, once used, the numerical results must be represented as dimensionless quantities. At this stage the only variable non-normalizable coefficient is $\mu$, 
which is used to label the sets of normalizable coefficients (A.21) and (A.29). At $\mu=0$, $\mathcal{V}_{B}$ vacuum is a Klebanov-Strassler solution, thus from (A.35),

$$
\epsilon=2^{5 / 4} 3^{-3 / 4} \exp \left(-\frac{1}{2}-\frac{3}{4} k_{s}\right)
$$

leading to (see (A.33))

$$
\begin{aligned}
& f_{a, 1,0}=2 \hat{\mathcal{Q}} \exp \left(-\frac{1}{3}-\frac{1}{2} k_{s}\right), \quad f_{a, 3,0}=2 \exp \left(-1-\frac{3}{2} k_{s}\right) \\
& k_{2,3,0}=-\left(2+3 k_{s}\right) \exp \left(-1-\frac{3}{2} k_{s}\right), \quad f_{a, 4,0}=-2 \hat{\mathcal{Q}} \exp \left(-\frac{4}{3}-2 k_{s}\right), \\
& f_{c, 4,0}=0, \quad g_{4,0}=0, \quad f_{a, 6,0}=-\frac{2}{25}\left(25 \hat{\mathcal{Q}}^{3}+15 k_{s}-14\right) \exp \left(-2-3 k_{s}\right), \\
& k_{2,7,0}=\frac{3}{2}\left(37-30 k_{s}\right) \hat{\mathcal{Q}}^{4} \exp \left(-\frac{7}{3}-\frac{7}{2} k_{s}\right) \\
& f_{a, 8,0}=-2 \hat{\mathcal{Q}}^{2}\left(\hat{\mathcal{Q}}^{3}+6 k_{s}-11\right) \exp \left(-\frac{8}{3}-4 k_{s}\right)
\end{aligned}
$$

in the UV, with (see (2.44))

$$
\hat{\mathcal{Q}} \equiv-\frac{4}{\sqrt{6}(2 \epsilon)^{2 / 3}} \mathcal{Q}=0.839917(9),
$$

and (see (A.34))

$$
\begin{array}{rlrl}
f_{a, 0}^{h} & =\frac{4}{3} 3^{2 / 3} \exp \left(-\frac{2}{3}-k_{s}\right), & h_{0}^{h} & =\frac{9}{16} 2^{2 / 3} \exp \left(\frac{4}{3}+2 k_{s}\right) \times 0.056288(0), \\
g_{0}^{h} & =1, \quad K_{1,3}^{h}=\exp \left(1+\frac{3}{2} k_{s}\right), K_{2,2}^{h}=\frac{1}{2} 3^{1 / 3} \exp \left(\frac{2}{3}+k_{s}\right), \\
K_{2,4}^{h} & =-\frac{11}{40} 3^{2 / 3} \exp \left(\frac{4}{3}+2 k_{s}\right), & K_{3,1}^{h} & =\frac{4}{9} 3^{2 / 3} \exp \left(\frac{1}{3}+\frac{1}{2} k_{s}\right), \quad f_{1,0}^{h}=1,
\end{array}
$$

in the IR.

In practice, fixing $k_{s}$, we start with $\mu=0$ with (5.19) and (5.21), and produce the data sets, incrementing $\mu$, for

$$
\frac{\mu}{\Lambda} \equiv 2^{-1 / 4} \mu \exp \left(\frac{1}{2} k_{s}\right)
$$

and $\hat{\mathcal{E}}$ of $(5.1)$, where $\Lambda \equiv 2^{1 / 4} \exp \left(-\frac{1}{2} k_{s}\right)$. A powerful numerical check is that the curves $\frac{\mu}{\Lambda}$ - versus $-\hat{\mathcal{E}}$ produced for different values of $k_{s}$ must collapse in the overlapping range of $\mu / \Lambda$. As figure 10 demonstrates, this is indeed what we find: the solid green curve is obtained with $k_{s}=\frac{1}{4}$ and the dashed magenta curve is obtained with $k_{s}=1$ - they differ at $\sim 10^{-5}$ fractional level or less. Yet a different computation scheme is to use the symmetry transformation (3.22) to keep $\mu=1$ fixed, and instead vary $K_{0}$, so that

$$
\frac{\mu}{\Lambda}=2^{-1 / 4} \exp \left(\frac{1}{2} K_{0}\right)
$$




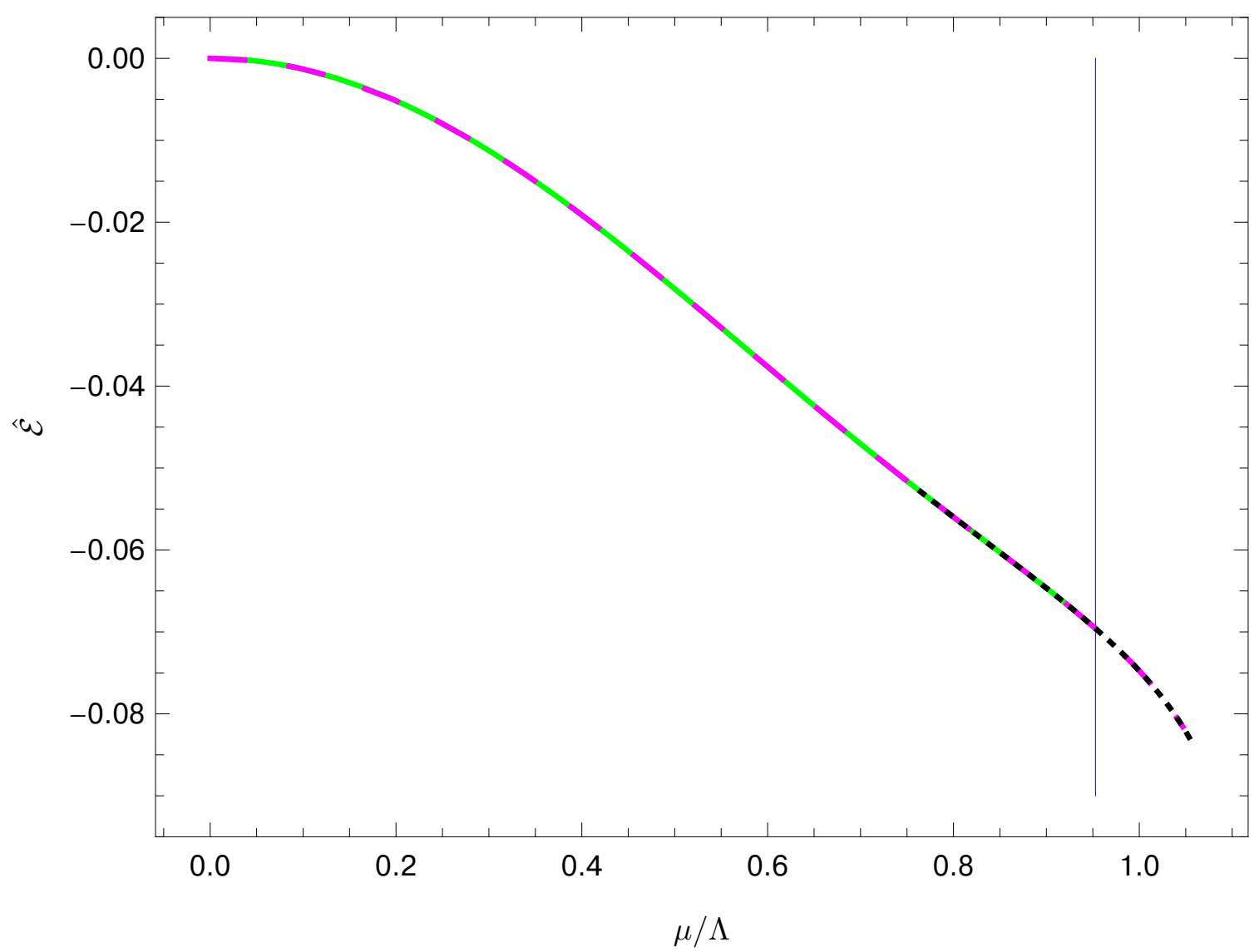

Figure 10. Cross checks on numerics of $\mathcal{V}_{B}$ vacua energy in the three different computational schemes: solid green curve, dashed magenta curve, and the dotted black curve. The vertical blue line denotes the common reference point in the green/black curve computational schemes, see (5.24).

Of course, in this computational scheme we can not connect to the Klebanov-Strassler $(\mu=0)$ solution, but we can connect to, say, green curve at $K_{0}=\frac{1}{4}$ and $\mu=1$, i.e., when

$$
\left.\frac{\mu}{\Lambda}\right|_{\text {connection to green }}=2^{-1 / 4} \exp \left(\frac{1}{2} \times \frac{1}{4}\right)=0.952860(5)
$$

which is represented by a vertical blue line. Such alternatively produced set of $\mathcal{V}_{B}$ vacua (dotted black curve in figure 10) must still agree with the green and magenta data sets. Clearly, this is the case: we extend the data sets for the black curve for both $K_{0}<\frac{1}{4}$ and $K_{0}>\frac{1}{4}$ to show the overlaps with the green/magenta data sets.

\section{$5.2 \mathcal{V}_{A}^{s}$ vacua}

These vacua were originally constructed in [10]. Equations of motion describing $\mathcal{V}_{A}^{s}$ vacua are collected in (A.1)-(A.10), which in the case of the unbroken chiral symmetry can be truncation as

$$
f_{b}(\rho) \equiv f_{a}(\rho) \equiv f_{3}(\rho), \quad K_{3}(\rho)=K_{1}(\rho) \equiv K(\rho), \quad K_{2}(\rho) \equiv 1
$$




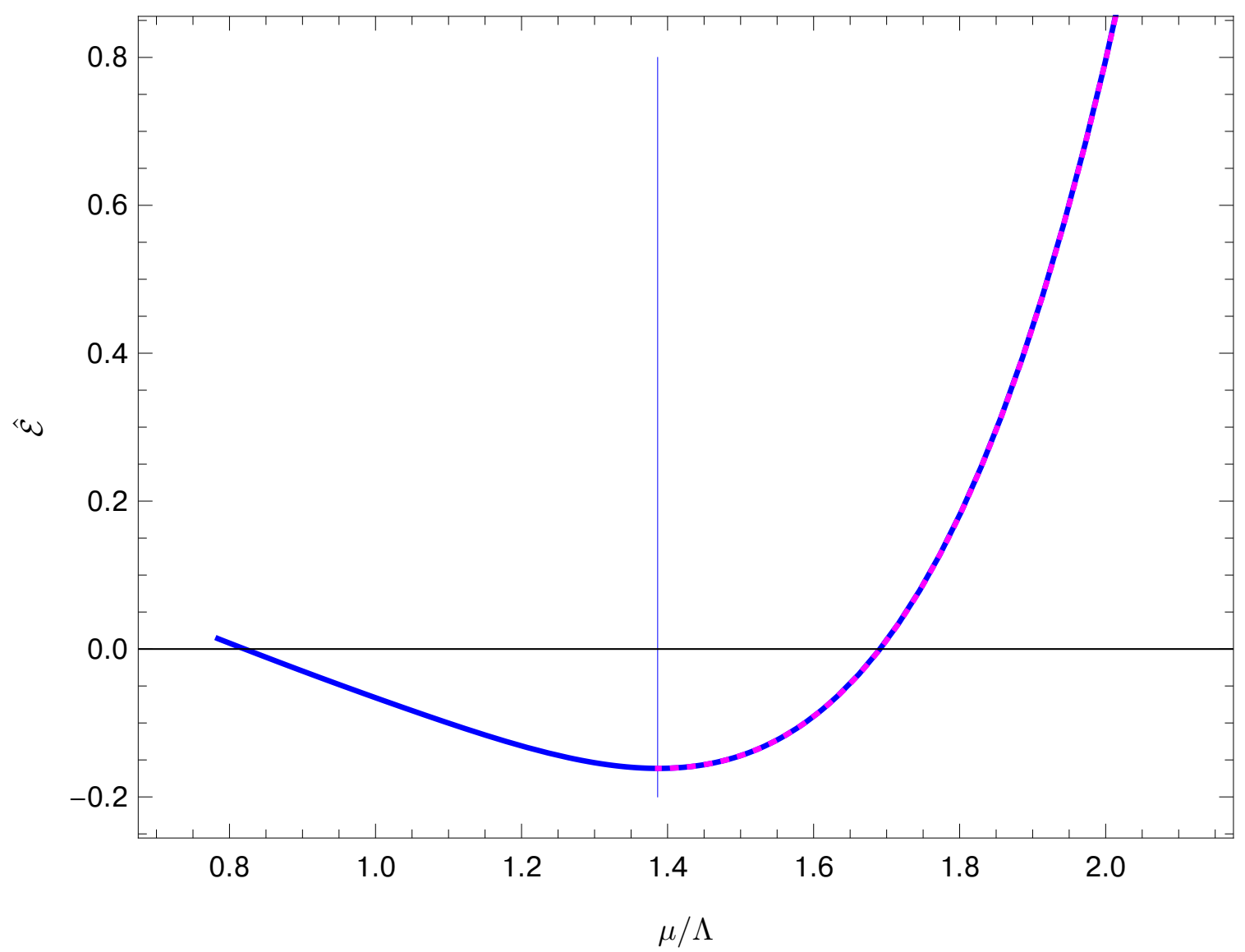

Figure 11. Cross checks on numerics of $\mathcal{V}_{A}^{s}$ vacua energy in the two different computational schemes: solid blue curve and the dotted magenta curve. The vertical blue line denotes the common reference point in the two computational schemes, see (5.31).

leading to nontrivial 5 second-order equations (A.1), (A.2), (A.5), (A.6), (A.9) and a single first order constraint (A.10). We have the UV, i.e., $\rho \rightarrow 0_{+}$, asymptotics (A.11)-(A.16), and the IR, i.e., $y \equiv \frac{1}{\rho} \rightarrow 0_{+}$, asymptotics (A.22), (A.23). There are 4 non-normalizable coefficients

$$
K_{0}, \mu, P, g_{s}
$$

characterizing the cascading gauge theory (A.20), and $17-6=11$ normalizable coefficients (A.21) and (A.24) - where the reduction in the number of the normalizable coefficients reflects the constraints of the unbroken chiral symmetry, see (A.26).

The numerical techniques for solving the required system of nonlinear ODEs were developed in [3]. We use the scaling symmetry (3.24) to set

$$
g_{s}=1
$$

We further use the symmetry transformation (3.22) to set ${ }^{12}$

$$
\mu=1 \text {. }
$$

\footnotetext{
${ }^{12}$ As before, this necessitates that the numerical results be represented as dimensionless quantities.
} 
The final symmetry transformation (3.25) is utilized to cross check the numerics:

- We can set $P=1$ and vary $K_{0}$, in which case

$$
\frac{\mu}{\Lambda} \equiv 2^{-1 / 4} \exp \left(\frac{1}{2} K_{0}\right) \quad \text { and } \quad \Lambda=2^{1 / 4} \exp \left(-\frac{1}{2} K_{0}\right) .
$$

- We can set $K_{0}=1$ and vary $P^{2} \equiv b$, in which case

$$
\frac{\mu}{\Lambda} \equiv 2^{-1 / 4} b^{1 / 2} \exp \left(\frac{1}{2 b}\right) \quad \text { and } \quad \Lambda=2^{1 / 4} b^{-1 / 2} \exp \left(-\frac{1}{2 b}\right) .
$$

Irrespectively which computational scheme is used, the produced curves $\frac{\mu}{\Lambda}-$ versus $-\hat{\mathcal{E}}$ must collapse in the overlapping range of $\mu / \Lambda$. As figure 11 demonstrates, this is indeed what we find: the solid blue curve is obtained keeping fixed $P=1$, and the dotted magenta curve is obtained keeping fixed $K_{0}=1$. The two schemes connect at $K_{0}=1$ and $b=1$, i.e., when

$$
\left.\frac{\mu}{\Lambda}\right|_{\text {connection }}=2^{-1 / 4} \exp \left(\frac{1}{2}\right)=1.38640(4),
$$

which is represented by a vertical blue line. ${ }^{13}$

\section{3 $\mathcal{V}_{A}^{b}$ vacua}

Analyzing normal modes about $\mathcal{V}_{A}^{s}$ vacua it was established in [10] that they are perturbatively unstable when $\mu<\mu_{u}$, see (5.4). However, no new branch of vacua with spontaneously broken symmetry, $\mathcal{V}_{A}^{b}$ in classification here, was identified. We use a different technique, introduced in [15], to independently reproduce the onset of the instability, and construct $\mathcal{V}_{A}^{b}$ vacua.

Consider static, chiral symmetry breaking fluctuations about $\mathcal{V}_{A}^{s}$ vacua:

$$
f_{a} \equiv f_{3}+\delta f, f_{b} \equiv f_{3}-\delta f, K_{1} \equiv K+\delta k_{1}, K_{3} \equiv K-\delta k_{1}, K_{2} \equiv 1+\delta k_{2},
$$

with the remaining metric functions and the string coupling as in $\mathcal{V}_{A}^{s}$ vacua $\left\{f_{1}, f_{c}, h, g\right\}$. It is straightforward to verify that truncation to $\left\{\delta f, \delta k_{1}, \delta k_{2}\right\}$ is consistent (at the linearized level). Equations of motion for the fluctuations and their asymptotic expansions in the $\mathrm{UV}(\rho \rightarrow 0)$ and the IR $\left(y=\frac{1}{\rho}\right)$ are collected in appendix B. Once the non-normalizable coefficient (the explicit chiral symmetry breaking parameter, i.e., the gaugino mass term) is fixed to $m=1$, the expansions are characterized by $6 \mathrm{UV} / \mathrm{IR}$ parameters

$$
\begin{aligned}
\mathrm{UV}: & \left\{\delta f_{3,0}, \delta k_{2,3,0}, \delta k_{2,7,0}\right\} ; \\
\mathrm{IR}: & \left\{\delta f_{0}^{h}, \delta k_{1,0}^{h}, \delta k_{2,0}^{h}\right\},
\end{aligned}
$$

which is the correct number of parameters to find a unique solution of 3 second-order differential equations (B.2)-(B.4) for $\left\{\delta f, \delta k_{1}, \delta k_{2}\right\}$ on $\mathcal{V}_{A}^{s}$ background parameterized by $K_{0}$ (it is convenient to use a computation scheme as in (5.29)).

\footnotetext{
${ }^{13}$ This is the smallest value of $\mu / \Lambda$ that can be reached in the computational scheme with $K_{0}=1$.
} 

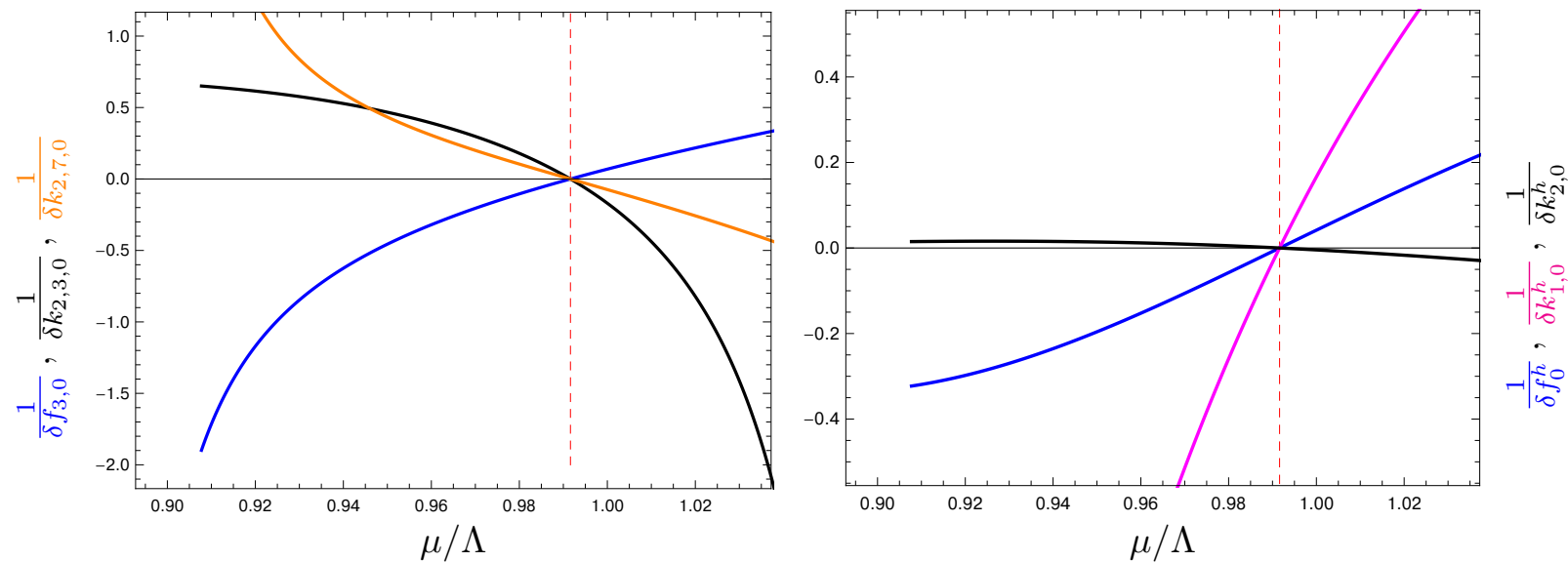

Figure 12. Parameters $\left\{\delta f_{3,0}, \delta k_{2,3,0}, \delta k_{2,7,0}, \delta f_{0}^{h}, \delta k_{1,0}^{h}, \delta k_{2,0}^{h}\right\}$ of the chiral symmetry breaking fluctuations over $\mathcal{V}_{A}^{s}$ vacua as a function of $\frac{\mu}{\Lambda}$, evaluated at fixed explicit chiral symmetry breaking scale $m=1$, diverge at $\mu=\mu_{u}(5.4)$, indicated by a vertical red dashed line. $\mu_{u}$ identifies the bifurcation point of spontaneous symmetry broken $\mathcal{V}_{A}^{b}$ vacua off chirally symmetric $\mathcal{V}_{A}^{s}$ vacua.
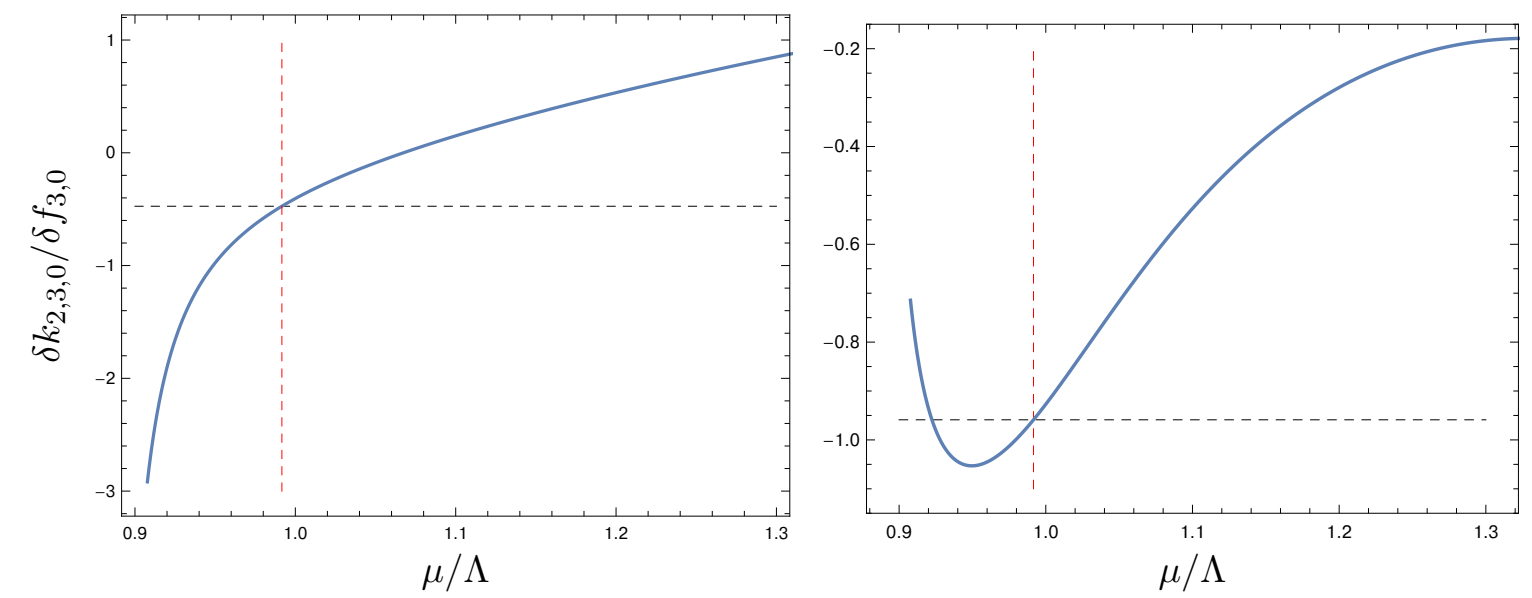

Figure 13. Susceptibilities of the UV parameters, see (5.35), of the linearized chiral symmetry breaking fluctuations. The red dashed vertical line denotes $\mu_{u}$, see (5.4). The black horizontal dashed lines indicate the values of the susceptibilities at $\mu=\mu_{\text {unstalble }}$, see (5.36).

In figure 12 we assemble results for the fluctuation parameters (5.33) as $K_{0}$ label of $\mathcal{V}_{A}^{s}$ vacua is varied. A signature of the spontaneous symmetry breaking is the divergence of all the parameters, once the scale of the explicit chiral symmetry breaking, the nonnormalizable parameter $m$, is kept fixed. This occurs at $\mu=\mu_{u}$ (5.4), represented by vertical dashed red lines. The corresponding critical value of $K_{0}$ is

$$
K_{0}^{\text {critical }}=0.329729(7)
$$



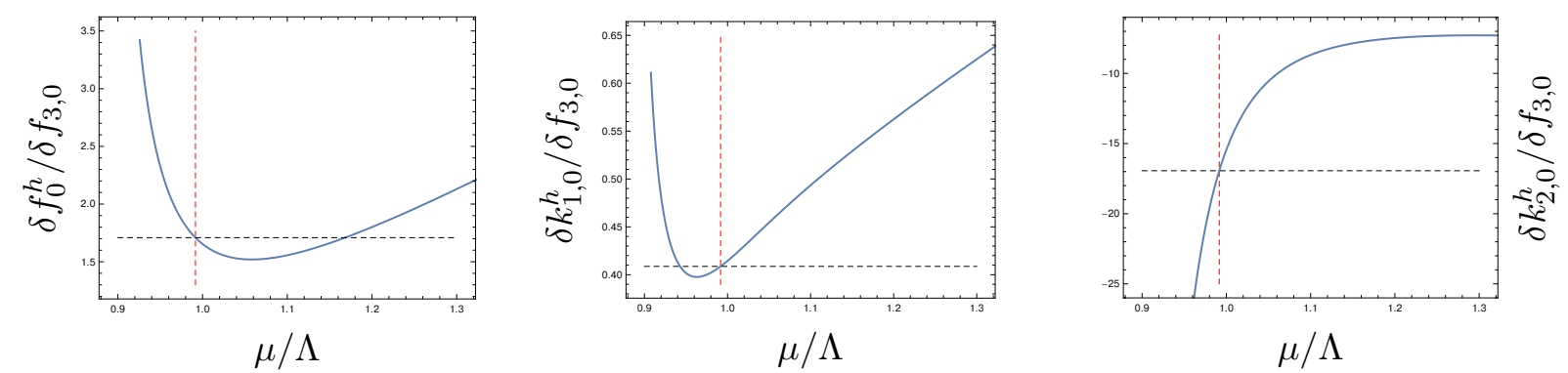

Figure 14. Susceptibilities of the IR parameters, see (5.35), of the linearized chiral symmetry breaking fluctuations. The red dashed vertical line denotes $\mu_{u}$, see (5.4). The black horizontal dashed lines indicate the values of the susceptibilities at $\mu=\mu_{\text {unstalble }}$, see (5.36).
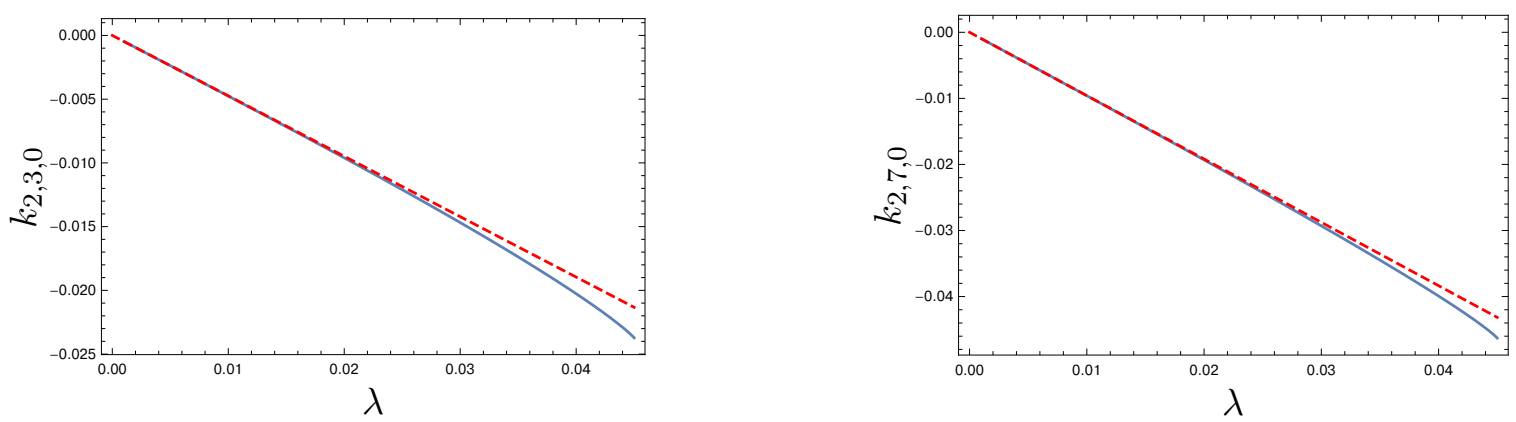

Figure 15. Sample of the UV parameters of $\mathcal{V}_{A}^{b}$ vacua constructed from the 'seed' (5.40). The linearized approximations in $\lambda$ are represented by dashed red lines.

To use the critical fluctuations as a seed for $\mathcal{V}_{A}^{b}$ vacua, we need to know the 'susceptibilities'

$$
\left\{\chi_{k_{2,3,0}}, \chi_{k_{2,7,0}}, \chi_{\hat{f}_{0}^{h}}, \chi_{k_{1,0}^{h}}, \chi_{k_{2,0}^{h}}\right\} \equiv \lim _{\mu \rightarrow \mu_{u}}\left\{\frac{\delta k_{2,3,0}}{\delta f_{3,0}}, \frac{\delta k_{2,7,0}}{\delta f_{3,0}}, \frac{\delta f_{0}^{h}}{\delta f_{3,0}}, \frac{\delta k_{1,0}^{h}}{\delta f_{3,0}}, \frac{\delta k_{2,0}^{h}}{\delta f_{3,0}}\right\}
$$

In figure 13 we present susceptibilities $\chi_{k_{2,3,0}}$ and $\chi_{k_{2,7,0}}$ - notice that they are finite at $\mu_{u}$, represented by vertical dashed red lines. The other susceptibilities (see figure 14) are finite as well; we find:

$$
\begin{aligned}
\chi_{k_{2,3,0}} & =-0.47398(9), \quad \chi_{k_{2,7,0}}=-0.95889(7), \quad \chi_{f_{0}^{h}}=1.7089(5), \\
\chi_{k_{1,0}^{h}} & =0.40872(4), \quad \chi_{k_{2,0}^{h}}=-16.936(2) .
\end{aligned}
$$

Given (5.36), fully nonlinear $\mathcal{V}_{A}^{b}$ vacua, with $K_{0}$ close to $K_{0}^{\text {crit }}$, can be constructed following the same procedure as the one employed in construction of Klebanov-Strassler black brane in [4]. We highlight the main steps: 

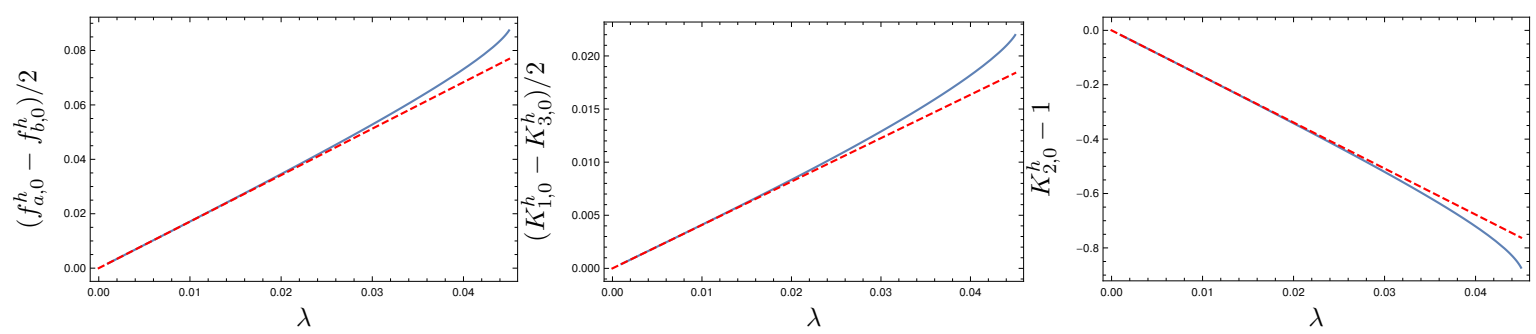

Figure 16. Sample of the IR parameters of $\mathcal{V}_{A}^{b}$ vacua constructed from the 'seed' (5.40). The linearized approximations in $\lambda$ are represented by dashed red lines.

- We set $K_{0}=K_{0}^{\text {crit }}$ and compute the corresponding $\mathcal{V}_{A}^{s}$ vacuum. This vacuum is characterized by (see (A.20), (A.21), (A.24) and (A.26))

$$
\begin{aligned}
& \mathrm{UV}:\left\{K_{0}=K_{0}^{\mathrm{crit}}, \mu=1, P=1, g_{s}=1, f_{a, 1,0}^{\mathrm{crit}}, f_{a, 3,0}^{\mathrm{crit}}=\frac{1}{4} f_{a, 1,0}^{\mathrm{crit}}, k_{2,3,0}^{\mathrm{crit}}=0,\right. \\
& \left.f_{a, 4,0}^{\text {crit }} \equiv f_{3,4,0}^{\text {crit }}, f_{c, 4,0}^{\text {crit }}, g_{4,0}^{\text {crit }}, f_{a, 6,0}^{\text {crit }}, k_{2,7,0}^{\text {crit }}=0, f_{a, 8,0}^{\text {crit }}\right\}, \\
& \text { IR : }\left\{f_{a, 0}^{h, \text { crit }} \equiv f_{3,0}^{h, \text { crit }}, f_{b, 0}^{h, \text { crit }} \equiv f_{3,0}^{h, \text { crit }}, f_{c, 0}^{h, \text { crit }}, h_{0}^{h, \text { crit }}, K_{1,0}^{h} \equiv K_{0}^{h, \text { crit }}, K_{2,0}^{h} \equiv 1,\right. \\
& \left.K_{3,0}^{h} \equiv K_{0}^{h, \text { crit }}, g_{0}^{h, \text { crit }}\right\} \text {. }
\end{aligned}
$$

- Let's denote the amplitude of the symmetry breaking condensate (see (5.32))

$$
\delta f_{3,0} \equiv \frac{1}{2}\left(f_{a, 3,0}-f_{b, 3,0}\right)=\lambda .
$$

Then,

$$
\left\{\delta k_{2,3,0}, \delta k_{2,7,0}, \delta f_{0}^{h}, \delta k_{1,0}^{h}, \delta k_{2,0}^{h}\right\}=\lambda\left\{\chi_{k_{2,3,0}}, \chi_{k_{2,7}, 0}, \chi_{f_{0}^{h}}, \chi_{k_{1,0}^{h}}, \chi_{k_{2,0}^{h}}\right\}+\mathcal{O}\left(\lambda^{2}\right) .
$$

- Using (5.32), (5.37)-(5.39) we find to $\mathcal{O}\left(\lambda^{2}\right)$ :

$$
\begin{aligned}
K_{0} & =K_{0}^{\text {crit }}+\mathcal{O}\left(\lambda^{2}\right), \quad f_{a, 1,0}=f_{a, 1,0}^{\text {crit }}+\mathcal{O}\left(\lambda^{2}\right), \quad f_{a, 3,0}=f_{a, 3,0}^{\text {crit }}+\lambda+\mathcal{O}\left(\lambda^{2}\right), \\
k_{2,3,0} & =k_{2,3,0}^{\text {crit }}+\lambda \chi_{k_{2,3,0}}+\mathcal{O}\left(\lambda^{2}\right), \quad f_{a, 4,0}=f_{a, 4,0}^{\text {crit }}+\mathcal{O}\left(\lambda^{2}\right), \quad f_{c, 4,0}=f_{c, 4,0}^{\text {crit }}+\mathcal{O}\left(\lambda^{2}\right), \\
g_{4,0} & =g_{4,0}^{\text {crit }}+\mathcal{O}\left(\lambda^{2}\right), \quad f_{a, 6,0}=f_{a, 6,0}^{\text {crit }}+\mathcal{O}\left(\lambda^{2}\right), \quad k_{2,7,0}=k_{2,7,0}^{\text {crit }}+\chi_{k_{2,7}, 0} \lambda+\mathcal{O}\left(\lambda^{2}\right), \\
f_{a, 8,0} & =f_{a, 8,0}^{\text {crit }}+\mathcal{O}\left(\lambda^{2}\right), \quad f_{a, 0}^{h}=f_{a, 0}^{h, \text { crit }}+\chi_{f_{0}^{h}} \lambda+\mathcal{O}\left(\lambda^{2}\right), \\
f_{b, 0}^{h} & =f_{b, 0}^{h, \text { crit }}-\chi_{f_{0}^{h}} \lambda+\mathcal{O}\left(\lambda^{2}\right), \quad f_{c, 0}^{h}=f_{c, 0}^{h, \text { crit }}+\mathcal{O}\left(\lambda^{2}\right), \quad h_{0}^{h}=h_{0}^{h, \text { crit }}+\mathcal{O}\left(\lambda^{2}\right), \\
K_{1,0}^{h} & =K_{1,0}^{h, \text { crit }}+\chi_{k_{1,0}^{h}} \lambda+\mathcal{O}\left(\lambda^{2}\right), \quad K_{2,0}^{h}=K_{2,0}^{h, \text { crit }}+\chi_{k_{2,0}^{h}} \lambda+\mathcal{O}\left(\lambda^{2}\right), \\
K_{3,0}^{h} & =K_{3,0}^{h, \text { crit }}-\chi_{k_{1,0}^{h}} \lambda+\mathcal{O}\left(\lambda^{2}\right), \quad g_{0}^{h}=g_{0}^{h, \text { crit }}+\mathcal{O}\left(\lambda^{2}\right) .
\end{aligned}
$$

- We construct fully nonlinear in $\lambda \mathcal{V}_{A}^{b}$ vacua using the linearized approximation (5.40) as a seed. Select UV/IR parameters, along with the corresponding linearized approximations (dashed red lines) are shown in figures 15-16. 


\section{Black holes on the warped deformed conifold with fluxes}

The physics of black holes on the warped deformed conifold with fluxes, correspondingly the dual deconfined thermal states of the cascading gauge theory plasma on $S^{3}$ with a radius $\frac{1}{\mu}$ is very rich. There are interesting phase transitions between them, as well as phase transitions towards confined thermal states. We set the stage by reviewing the results in the decompactification limit $\frac{\mu}{\Lambda} \rightarrow 0$, followed by the general presentation for $\frac{\mu}{\Lambda} \neq 0$. We highlight details in the following subsections, and delegate the numerical checks to the appendix E. The $\mu=0$ results were obtained over the years in $[3,4,11,14,23,25,37-40]$. All the $\mu \neq 0$ results are new.

\subsection{Conifold black branes and phase transitions at $\frac{\mu}{\Lambda}=0$}

At $\mu=0$ we have (see figure 3 ):

- $\mathcal{T}_{\text {decon }}^{s}$ : Klebanov-Tseytlin black branes, representing thermal deconfined states of the cascading gauge theory plasma with the unbroken chiral symmetry (blue / brown curves in the figures below);

- $\mathcal{T}_{\text {decon }}^{b}$ : Klebanov-Strassler black branes, representing thermal deconfined states of the cascading gauge theory plasma with spontaneously broken chiral symmetry (magenta curves in the figures below);

- $\mathcal{T}_{\text {con,B }}$ : confined states with spontaneously broken chiral symmetry (green curves in the figures below).

Similar to (5.1), it is convenient to introduce the reduced free energy density $\hat{\mathcal{F}}$, and the reduced entropy density $\hat{s}$ :

$$
\hat{\mathcal{F}} \equiv \frac{8 \pi G_{5}}{P^{4} g_{s}^{2}} \frac{\mathcal{E}}{\Lambda^{4}}=\frac{2^{6} \pi^{4}}{3^{5} M^{4}} \frac{\mathcal{E}}{\Lambda^{4}}, \quad \hat{s} \equiv \frac{8 \pi G_{5}}{P^{4} g_{s}^{2}} \frac{s}{\Lambda^{3}}=\frac{2^{6} \pi^{4}}{3^{5} M^{4}} \frac{s}{\Lambda^{3}}
$$

In figure 17 we recall the phase diagram in the canonical ensemble. There are three critical temperatures

$$
T_{u}<T_{\chi \mathrm{SB}}<T_{c} .
$$

$T_{c}$ is the temperature of the confinement-deconfinement phase transition [3], i.e., the transition $\mathcal{T}_{\text {decon }}^{s} \leftrightarrow \mathcal{T}_{\text {con, } \mathrm{B}}$

$$
T_{c}=0.614(1) \Lambda
$$

The confined phase with spontaneously broken chiral symmetry $\mathcal{T}_{\text {con,B }}$ has a lower free energy density for $T<T_{c}$, while the chirally symmetric deconfined phase $\mathcal{T}_{\text {decon }}^{s}$ dominates when $T>T_{c}$. Since the entropy density of $\mathcal{T}_{\text {decon }}^{s}$ states $\hat{s} \neq 0$, while the reduced entropy density of $\mathcal{T}_{\text {con }}^{b}$ states identically vanishes, ${ }^{14}$ the phase transition at $T=T_{c}$ is of the first-order.

\footnotetext{
${ }^{14}$ This is true in the supergravity, i.e., the large- $M$ approximation.
} 


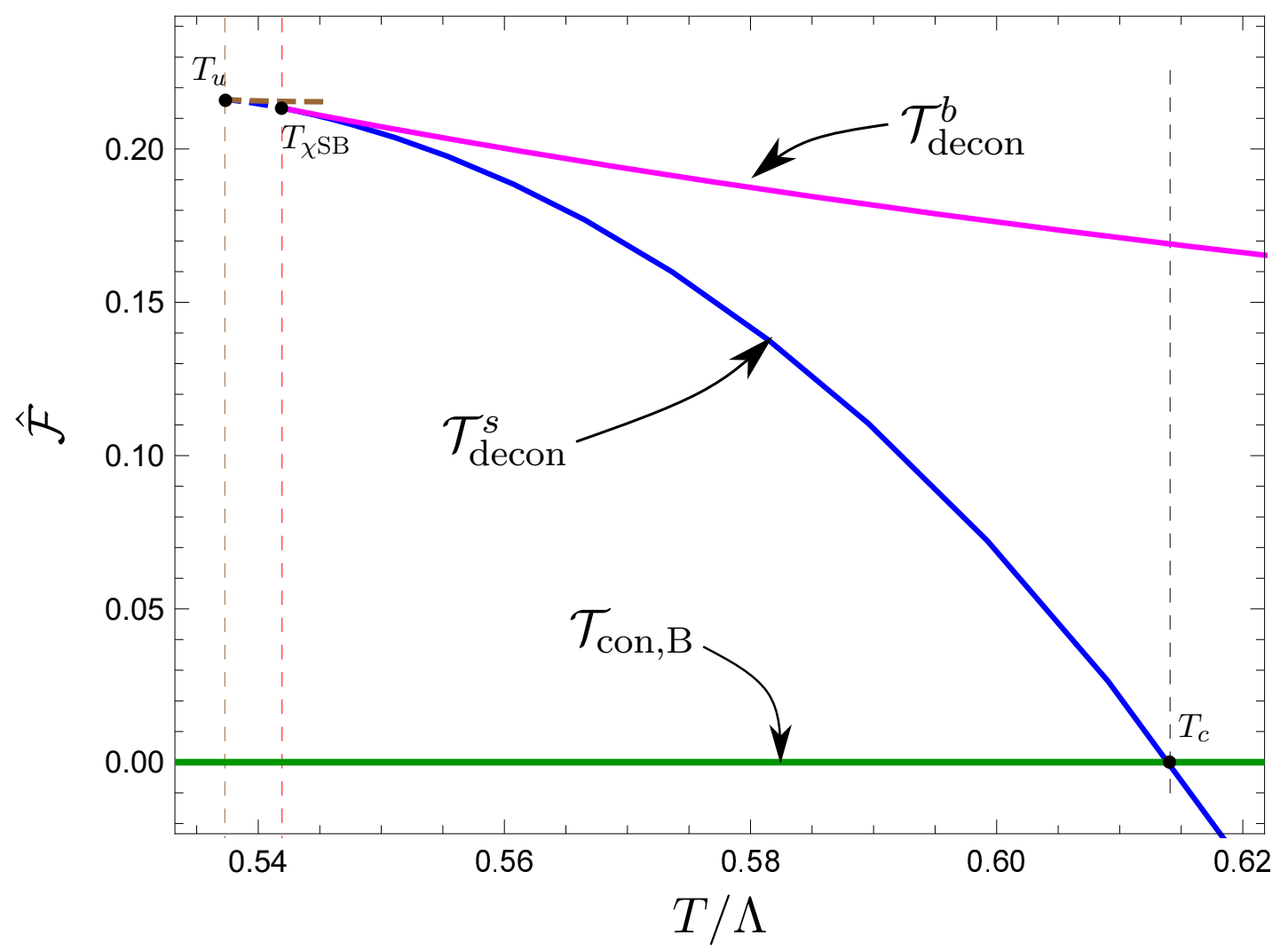

Figure 17. Phase diagram in the canonical ensemble at $\mu / \Lambda=0$ : the reduced free energy density $\hat{\mathcal{F}}$, see (6.1), versus the reduced temperature $T / \Lambda$ for different states in the theory. Vertical dashed lines indicate critical temperatures $T_{c}$ (black) for the confinement-deconfinement phase transition, $T_{\chi \mathrm{SB}}$ (red) for the onset of the spontaneous chiral symmetry breaking, and $T_{u}$ (brown) for the bifurcation point of the $\mathcal{T}_{\text {decon }}^{s}$ states with positive/negative specific heat.
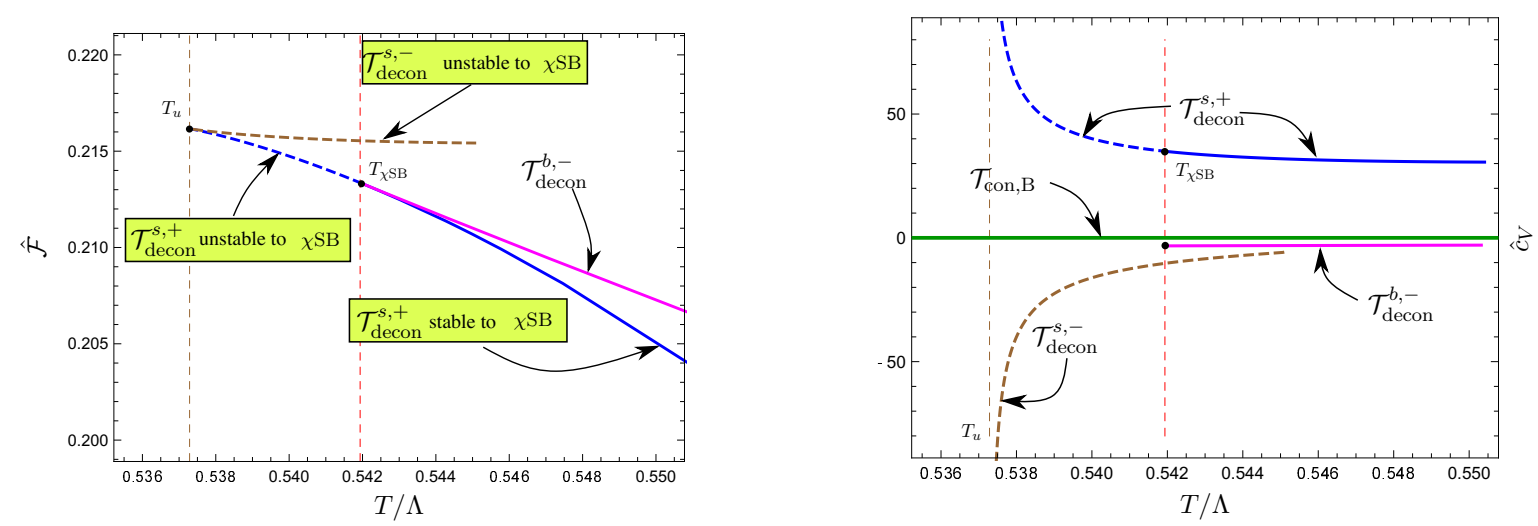

Figure 18. Left panel: detailed phase diagram in the canonical ensemble at $\mu / \Lambda=0$ for $T \gtrsim T_{u}$. $\mathcal{T}_{\text {decon }}^{s}$ states unstable to spontaneous chiral symmetry breaking are denoted with dashed curves. Right panel: specific heat $\hat{c}_{V}$ (6.6) of different states. Brown curves denote $\mathcal{T}_{\text {decon }}^{s}$ states with $\hat{c}_{V}<0$, and blue curves denote $\mathcal{T}_{\text {decon }}^{s}$ states with $\hat{c}_{V}>0$. Note that the specific heat of $\mathcal{T}_{\text {decon }}^{b}$ states (magenta curves), i.e., the Klebanov-Strassler black branes, is negative. 
$\mathcal{T}_{\text {decon }}^{s}$ phase is stable with respect to linearized (perturbative) chiral symmetry breaking fluctuations when $T>T_{\chi \mathrm{SB}}[11]$

$$
T_{\chi \mathrm{SB}}=0.541(9) \Lambda
$$

and is unstable (denoted by the dashed curves) when $T<T_{\chi \mathrm{SB}} . T=T_{\chi \mathrm{SB}}$ is a bifurcation point of the phase diagram, where deconfined phase with spontaneously broken chiral symmetry $\mathcal{T}_{\text {decon }}^{b}$ joins the symmetric $\mathcal{T}_{\text {decon }}^{s}$ phase [4]. This phase, represented by the Klebanov-Strassler black branes, exists for $T>T_{\chi \mathrm{SB}}$, but never dominates in the canonical ensemble as it has a higher free energy density than that of the chirally symmetric phase $\mathcal{T}_{\text {decon }}^{s}$ at the same temperature.

$T_{u}[39]$,

$$
T_{u}=0.537(3) \Lambda,
$$

is the terminal temperature of the $\mathcal{T}_{\text {decon }}^{s}$ states. It is the second bifurcation point on the phase diagram, which separates deconfined chirally symmetric states with positive specific heat $\hat{c}_{V}$,

$$
\hat{c}_{V} \equiv \Lambda \frac{d \hat{\mathcal{E}}}{d T}
$$

denoted as $\mathcal{T}_{\text {decon }}^{s,+}$ (blue curves) in figure 18, from deconfined chirally symmetric states with a negative specific heat $\mathcal{T}_{\text {decon }}^{s,-}$ (brown curves). At a temperature where both $\mathcal{T}_{\text {decon }}^{s,+}$ and $\mathcal{T}_{\text {decon }}^{s,-}$ states exist, the former ones always have a lower free energy density. Note that the specific heat of the deconfined states with the spontaneously broken chiral symmetry is also negative, thus these states are denoted as $\mathcal{T}_{\text {decon }}^{b,-}$ (magenta curves) in figure 18.

Deconfined states of the cascading gauge theory plasma at $\mu / \Lambda=0$ are dual to black branes: the holographic geometries with a regular Schwarzschild horizon with translational invariance. It was pointed out in [41] that a negative specific heat of the black branes immediately implies that the extended horizon is perturbatively unstable to metric fluctuations, breaking the translational invariance. The latter is a holographic dual to the fact that in an infinitely spatially extended media with a negative specific heat the speed of the sound waves is purely imaginary:

$$
c_{s}^{2}=\frac{s}{c_{V}}<0 \quad \text { if } \quad c_{V}<0 .
$$

Thus, the states $\mathcal{T}_{\text {decon }}^{s,-}$ and all the $\mathcal{T}_{\text {decon }}^{b}$ states are perturbatively unstable to inhomogeneous metric fluctuations.

In figure 19 we discuss the criticality of $\mu / \Lambda=0$ states at $T=T_{u}$ (the left panel) and $T=T_{\chi \mathrm{SB}}$ (the right panel). In the former case, both for $\mathcal{T}_{\text {decon }}^{s,-}$ and $\mathcal{T}_{\text {decon }}^{s,+}$ states

$$
\hat{\mathcal{E}}^{ \pm}-\hat{\mathcal{E}}_{u} \propto \sqrt{T-T_{u}} \quad \Longrightarrow \quad c_{V}^{ \pm} \propto\left(T-T_{u}\right)^{-1 / 2},
$$

implying that the specific heat diverges as $T \rightarrow T_{u}$ with the critical exponent ${ }^{15} \alpha=1 / 2$. Of course, this is consistent with the results of the right panel in figure 18. In the vicinity

\footnotetext{
${ }^{15}$ In holographic models such critical behavior was identified first in [42].
} 

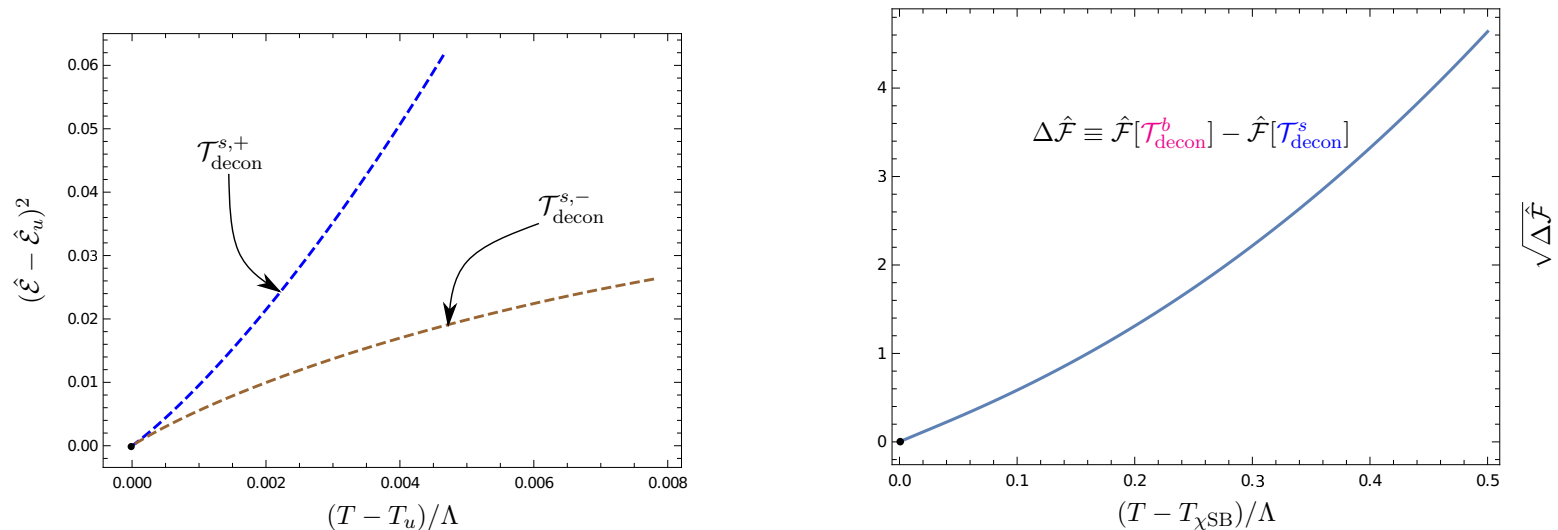

Figure 19. Critical behavior at $T=T_{u}$ (the left panel) and $T=T_{\chi \mathrm{SB}}$ (the right panel). In the former case, the specific heat of $\mathcal{T}_{\text {decon }}^{s, \pm}$ states diverge as $T \rightarrow T_{u+}$ with the critical exponent $\alpha=\frac{1}{2}$. The scaling of the free energy density difference between the two phase as $T \rightarrow T_{\chi \mathrm{SB}}+$ implies that the phase transition at $T=T_{\chi \mathrm{SB}}$ is of the second order.

of $T=T_{\chi \mathrm{SB}}$, the free energy density difference $\Delta \hat{\mathcal{F}}$, between the deconfined states with the spontaneously broken $\left(\mathcal{T}_{\text {decon }}^{b}\right)$ and the unbroken $\left(\mathcal{T}_{\text {decon }}^{s}\right)$ chiral symmetry, scales as

$$
\Delta \hat{\mathcal{F}} \propto\left(T-T_{\chi \mathrm{SB}}\right)^{2},
$$

as indicative of the second-order phase transition.

Figure 20 represents the phase diagram of the system at $\mu / \Lambda=0$ in the microcanonical ensemble. Microcanonical ensemble is crucial in understanding the dynamics and the equilibration of the generic states in the theory. We will omit discussion of the confined states as the latter have vanishing reduced entropy density $\hat{s}(6.1)$ and thus never dominate over the deconfined states in the microcanonical ensemble. There are two critical energy densities (corresponding to critical temperatures $T_{\chi \mathrm{SB}}$ and $T_{u}$ in the canonical ensemble)

$$
\hat{\mathcal{E}}_{u}<\hat{\mathcal{E}}_{\chi \mathrm{SB}} .
$$

$\hat{\mathcal{E}}_{\chi \mathrm{SB}}$

$$
\hat{\mathcal{E}}_{\chi \mathrm{SB}}=0.635(1),
$$

is the critical energy density below which $\mathcal{T}_{\text {decon }}^{s}$ (represented by the dashed curves) become unstable to perturbative chiral symmetry breaking instability. $\mathcal{T}_{\text {decon }}^{b}$ states, represented by the Klebanov-Strassler black branes [4] - the magenta curve in figure 20, exist for $\hat{\mathcal{E}}<\hat{\mathcal{E}}_{\chi \mathrm{SB}}$ and are more entropic (and thus are the dominant ones) compare to $\mathcal{T}_{\text {decon }}^{s}$ states, represented by the Klebanov-Tseytlin black branes [3, 23, 37, 38]. While thermodynamic instability, i.e., the negative specific heat, in general does not affect the phase diagram in the microcanonical ensemble, it does so when $\mu / \Lambda=0$ as in this case it is related to the perturbative instability breaking the translational invariance of the thermal states - the purely imaginary speed of the sound waves in the cascading gauge theory interpretation. Since $c_{s}^{2}<0$ for $\mathcal{T}_{\text {decon }}^{b}$ states (at all energy densities), they can not be the end point of the evolution of the chiral symmetry breaking instability in $\mathcal{T}_{\text {decon }}^{s}$ states. Neither can $\mathcal{T}_{\text {con, B }}$ states 


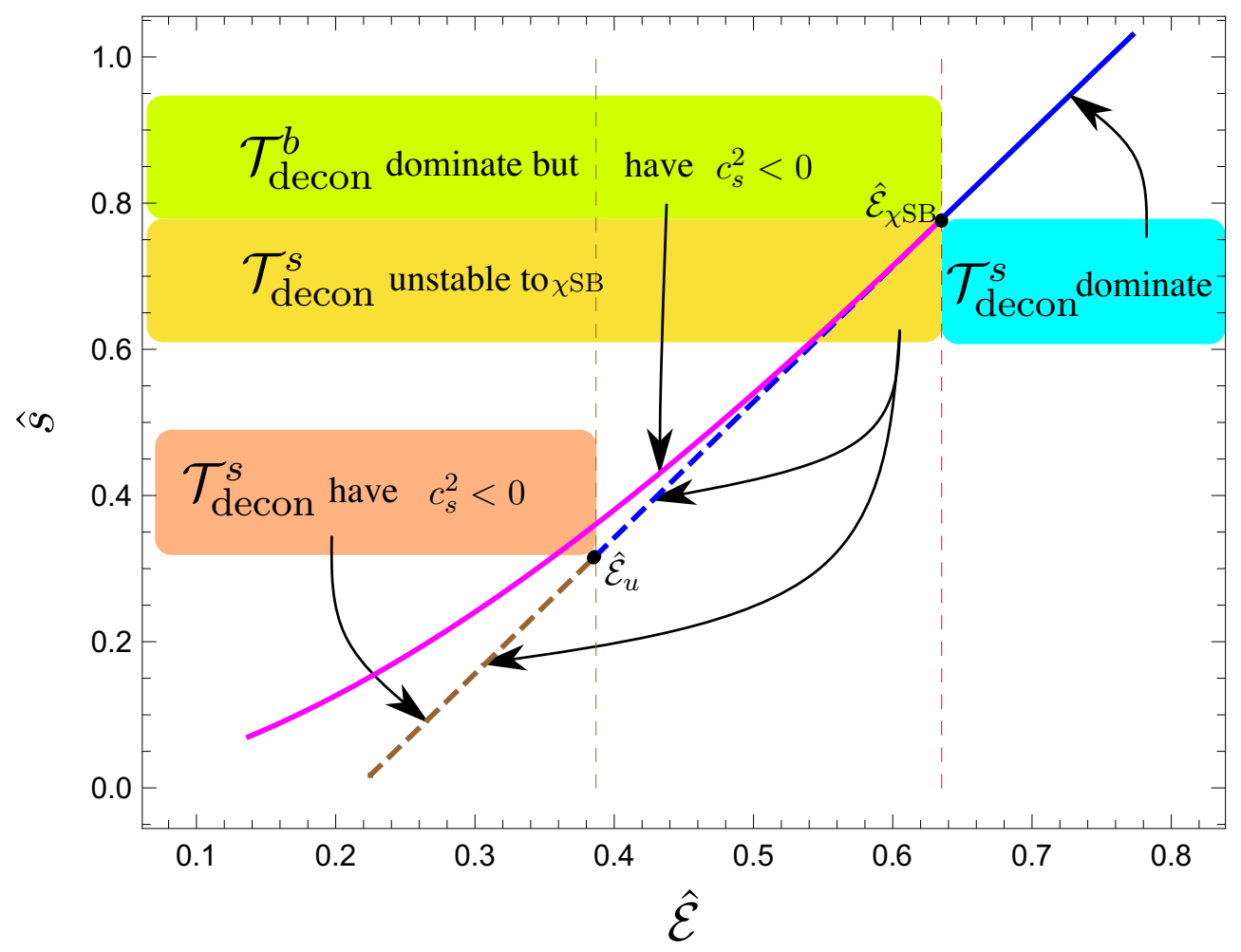

Figure 20. Phase diagram in the microcanonical ensemble at $\mu / \Lambda=0$ : the reduced entropy density $\hat{s}$, see (6.1), versus the reduced energy density $\hat{\mathcal{E}}$, see (5.1), for different deconfined states in the theory. Vertical dashed lines indicate critical reduced energy densities $\hat{\mathcal{E}}_{\chi \mathrm{SB}}$ (red) for the of the spontaneous chiral symmetry breaking, and $\hat{\mathcal{E}}_{u}$ (brown) for the onset of the spontaneous breaking of the spatial translational invariance in $\mathcal{T}_{\text {decon }}^{s}$ states.

be the end point of this instability, as these are the confined states. Identification of the end point of the chiral symmetry breaking instability in $\mathcal{T}_{\text {decon }}^{s}$ states remains an open problem. $\hat{\mathcal{E}}_{u}$

$$
\hat{\mathcal{E}}_{u}=0.386(9)
$$

is the critical energy density that separates $\mathcal{T}_{\text {decon }}^{s}$ states stable to spontaneous breaking of the spatial translational invariance (blue curves with $\hat{\mathcal{E}}>\hat{\mathcal{E}}_{u}$ ), from the states where it is broken (brown curve).

From figure $20, \mathcal{T}_{\text {decon }}^{b}$ states exist only for $\hat{\mathcal{E}}<\hat{\mathcal{E}}_{\text {XSB }}$, while $\mathcal{T}_{\text {decon }}^{s}$ extend to arbitrary high energy densities. ${ }^{16}$ Neither $\mathcal{T}_{\text {decon }}^{s}$ nor the $\mathcal{T}_{\text {decon }}^{b}$ states reach to the energy density of the true vacuum, see (A.85),

$$
\left.\hat{\mathcal{E}}\left[\mathcal{V}_{B}\right]\right|_{\mu / \Lambda=0}=0
$$

Figure 21 explains why that is the case. In the left panel we present the Kretschmann scalar $\hat{\mathcal{K}}$ (5.7) for different deconfined states in the theory normalized to its value at $\hat{\mathcal{E}}=\hat{\mathcal{E}}_{\chi \mathrm{SB}}$, i.e., $\hat{\mathcal{K}}_{\chi \mathrm{SB}}$. Note the sharp increase in $\hat{\mathcal{K}} / \hat{\mathcal{K}}_{\chi \mathrm{SB}}$ as $\hat{\mathcal{E}}<\hat{\mathcal{E}}_{\chi \mathrm{SB}}$. For $\mathcal{T}_{\text {decon }}^{b}$ states (magenta

\footnotetext{
${ }^{16}$ We explicitly construct these states for $\hat{\mathcal{E}} \rightarrow \infty$ in section 6.4 .
} 

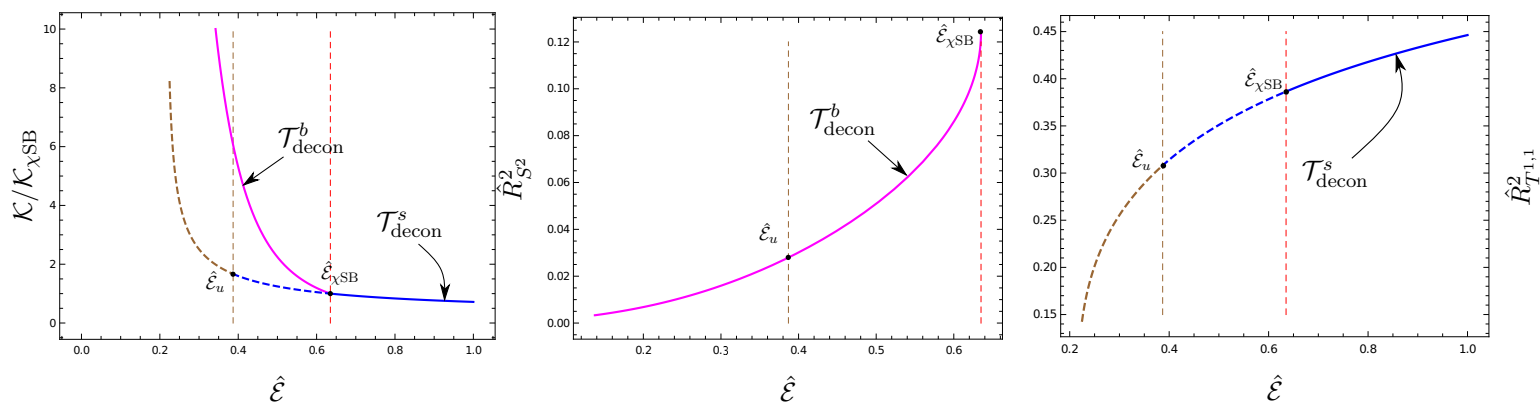

Figure 21. Left panel: the reduced Kretschmann scalar $\hat{\mathcal{K}}$ (5.7) of the holographic geometries dual to $\mu / \Lambda=0$ deconfined stated of the theory normalized to $\hat{\mathcal{K}}_{\chi \mathrm{SB}}$. The small $\hat{\mathcal{E}}$ growth of $\hat{\mathcal{K}}$ of $\mathcal{T}_{\text {decon }}^{b}$ states is associated with collapsing of the conifold 2-cycle (the central panel), and of $\mathcal{T}_{\text {decon }}^{s}$ states is associated with collapsing of the deformed $T^{1,1}$ (the right panel).

curve, the central panel) this growth is associated with the collapse of the conifold 2-cycle, see (5.13). For $\mathcal{T}_{\text {decon }}^{s}$ states (blue/brown curves, the right panel) this growth is associated with the collapse of the deformed $T^{1,1}$, see (5.11) and (5.12).

\subsection{Conifold black holes and phase transitions at $\frac{\mu}{\Lambda} \neq 0$}

In section 6.2.1 we presents the results for the conifold black holes in the canonical ensemble, followed by the microcanonical ensemble discussion in section 6.2.2. In section 6.2.3 we dive into details of the black holes thermodynamics for select values $\mu \neq 0$, highlighting similarities and differences with the $\mu=0$ case covered in section 6.1.

The main conceptual difference with the black branes on the conifold, reviewed in section 6.1, is that the black hole horizon is compact; as the result, there is no simple relation between the thermodynamic and the dynamic instabilities. While we do identify branches of the black holes with the negative specific heat, this does not imply that these black holes are unstable. The stability analysis of the black holes with respect to perturbative metric fluctuations breaking the spatial $\mathrm{SO}(4)$ symmetry will be discussed elsewhere [43].

\subsubsection{Canonical ensemble}

The critical temperatures of the canonical ensemble phase diagram identified in section 6.1 become functions of $\mu / \Lambda$; the hierarchy of scales (6.2) is however preserved:

$$
T_{u}(\mu) \leq T_{\chi \mathrm{SB}}(\mu)<T_{c}(\mu) .
$$

There is a special value $\mu^{\star}$, such that

$$
T_{u}\left(\mu^{\star}\right)=T_{\chi \mathrm{SB}}\left(\mu^{\star}\right) \quad \Longrightarrow \quad \mu^{\star}=0.848(0) \Lambda .
$$

Similar to the black branes, at $\mu / \Lambda \neq 0$ :

- The critical temperature $T_{u}(\mu)$ identifies the terminal temperature of $\mathcal{T}_{\text {decon }}^{s}$ states. This temperature is a bifurcation point for the two branches of the Klebanov-Tseytlin black holes, the $\mathcal{T}_{\text {decon }}^{s}$ states, with the positive and the negative reduced specific heat densities $\hat{c}_{V}$, see (6.6). We use blue curves to refer to $\mathcal{T}_{\text {decon }}^{s,+}$ states with $\hat{c}_{V}>0$, and 
brown curves when the reduced specific heat density of the $\mathcal{T}_{\text {decon }}^{s,-}$ states is negative. As $T \rightarrow T_{u}(\mu)_{+}$, the reduced specific heat diverges with the same critical exponent as for $\mu=0$, see (6.8). We find that for all values of $\mu / \Lambda$, at an appropriate ${ }^{17}$ fixed temperature, $\mathcal{T}_{\text {decon }}^{s,+}$ states have a smaller reduced free energy densities $\hat{\mathcal{F}}(6.1)$ then that of the $\mathcal{T}_{\text {decon }}^{s,-}$ states.

- The critical temperature $T_{\chi \mathrm{SB}}(\mu)$ (red curve) denotes the onset of the perturbative chiral symmetry breaking instability of the $\mathcal{T}_{\text {decon }}^{s}$ states. $T_{\chi \mathrm{SB}}(\mu)$ is also the bifurcation point on the phase diagram, where $\mathcal{T}_{\text {decon }}^{s}$ and $\mathcal{T}_{\text {decon }}^{b}$ branches of states join with the second-order phase transition.

- The reduced specific heat of the $\mathcal{T}_{\text {decon }}^{b}$ states, or the Klebanov-Strassler black holes (represented with magenta curves), is always negative.

On the contrary to the black branes, at $\mu / \Lambda \neq 0$ :

- $\mathcal{T}_{\text {decon }}^{s,-}$ states, while having a negative specific heat, can be perturbatively stable to the chiral symmetry breaking fluctuations, provided $\mu>\mu^{\star}$, see (6.15).

- Related to above, while at $\mu=0$ the chiral symmetry is spontaneously broken for $T<T_{\chi \mathrm{SB}}$, for $\mu>\mu^{\star}$, the chiral symmetry breaking occurs for $T>T_{\chi \mathrm{SB}}(\mu)$ in $\mathcal{T}_{\text {decon }}^{\text {s, }}$ states. In this regime, at a fixed temperature, the reduced free energy density of the symmetry broken states $\mathcal{T}_{\text {decon }}^{b}$ is lower than that of the $\mathcal{T}_{\text {decon }}^{s,-}$, however it is still larger than the reduced free energy density of the $\mathcal{T}_{\text {decon }}^{s,+}$ states at the corresponding temperature, i.e.,

$$
\mu>\mu^{\star}: \quad \hat{\mathcal{F}}\left[\mathcal{T}_{\text {decon }}^{s,+}\right]<\hat{\mathcal{F}}\left[\mathcal{T}_{\text {decon }}^{b}\right]<\hat{\mathcal{F}}\left[\mathcal{T}_{\text {decon }}^{s,-}\right]
$$

- There is a conceptual difference associated with the critical temperature $T_{c}(\mu)$ for the confinement-deconfinement phase transitions, albeit all these phase transitions are still of the first order. At $\mu=0$ this transition is between $\mathcal{T}_{\text {decon }}^{s}$ and $\mathcal{T}_{\text {con, B }}$ states. For $\mu / \Lambda \neq 0$, at a given value of $\mu$, there can exist two or even three different confined states: ${ }^{18} \mathcal{T}_{\text {con, } \mathrm{B}}$ and $\mathcal{T}_{\text {con, } \mathrm{A}}^{s}$ when $\mu$ is large enough but smaller than $\mu_{\chi \mathrm{SB}}$, or $\mathcal{T}_{\text {con, } \mathrm{B}}$, $\mathcal{T}_{\text {con, } \mathrm{A}}^{s}$ and $\mathcal{T}_{\text {con }, \mathrm{A}}^{b}$ for $\mu \gtrsim \mu_{\chi \mathrm{SB}}$. As a result, we must augment the $T_{c}(\mu)$ label with the corresponding confined state label. We use green curves to denote confinementdeconfinement transitions $\mathcal{T}_{\text {decon }}^{s} \leftrightarrow \mathcal{T}_{\text {con, } \mathrm{B}}$, blue curves to denote transitions $\mathcal{T}_{\text {decon }}^{s} \leftrightarrow$ $\mathcal{T}_{\text {con, } \mathrm{A}}^{s}$, and magenta curves to denote transitions $\mathcal{T}_{\text {decon }}^{s} \leftrightarrow \mathcal{T}_{\text {con,A }}^{b}$. Much like $\mathcal{V}_{A}^{s}$ vacua (see figure 5), $\mathcal{T}_{\text {con,A }}^{s}$ states are perturbatively unstable to chiral symmetry breaking fluctuations for $\mu<\mu_{u}$. We represent branches of $T_{c}(\mu)$ subject to such instabilities with dashed curves.

In figure 22 we present the temperature of the first-order phase confinement / deconfinement phase transition $T_{c}$ as a function of $\mu$ between $\mathcal{T}_{\text {decon }}^{s}$ and $\mathcal{T}_{\text {con,B }}$ states (the

\footnotetext{
${ }^{17}$ Both states $\mathcal{T}_{\text {decon }}^{s,+}$ and $\mathcal{T}_{\text {decon }}^{s,-}$ must exist.

${ }^{18}$ Figures 5 and 6 make this point obvious.
} 


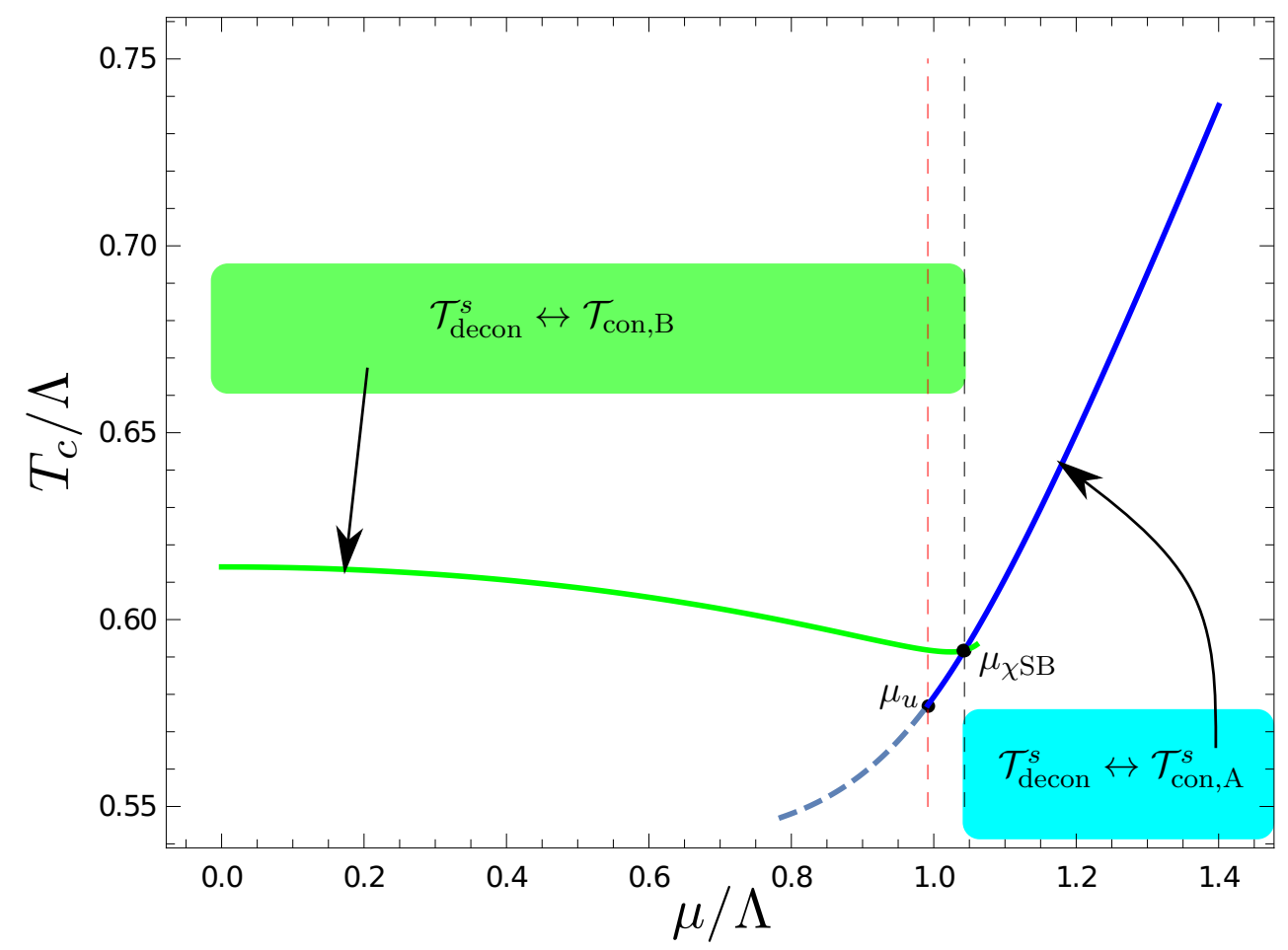

Figure 22. The temperature $T_{c}$ of the confinement/deconfinement phase transitions between the different states in the cascading gauge theory; equivalently, the analog of the Hawking-Page phase transition between the Klebanov-Tseytlin black holes and the different Euclidean vacua of the conifold flux geometries. The deconfined states are the preferred ones for $T>T_{c}$.

green curve) and between $\mathcal{T}_{\text {decon }}^{s}$ and $\mathcal{T}_{\text {con }, \mathrm{A}}^{s}$ states (the blue curves). The $\mathcal{T}_{\text {decon }}^{s} \leftrightarrow \mathcal{T}_{\text {con, } \mathrm{B}}$ transition is the finite $\mu$ extension of the phase transition at $\mu=0$ identified originally in [3]. Note that for $\mu>\mu_{\chi \mathrm{SB}}$ the confined states $\mathcal{T}_{\text {con,A }}^{s}$ are thermodynamically referred, compare to $\mathcal{T}_{\text {con,B }}$ states. ${ }^{19}$ For $\mu<\mu_{u}$ the confined states $\mathcal{T}_{\text {con,A }}^{s}$ are perturbatively unstable with respect to chiral symmetry breaking fluctuations (represented with the dashed curve). The blue curve extends to $\mu / \Lambda \rightarrow \infty$ reproducing the Hawking-Page transition of the $A d S_{5} \times T^{1,1}$ black holes, see section 4.2 .

In figure 23 we present the temperature of the first-order phase confinement/deconfinement phase transition $T_{c}$ as a function of $\mu$ between $\mathcal{T}_{\text {decon }}^{s}$ and $\mathcal{T}_{\text {con, A }}^{s}$ states (the blue curve) and between $\mathcal{T}_{\text {decon }}^{s}$ and $\mathcal{T}_{\text {con,A }}^{b}$ states (the magenta curve). Note that these phase transitions are never realized in practice since for the range of $\mu / \Lambda$ in question the preferred phase is always $\mathcal{T}_{\text {con, } \mathrm{B}}$, compare with figure 22 .

In figure 24 we present that temperature of the confinement/deconfinement transition $\mathcal{T}_{\text {decon }}^{s} \leftrightarrow \mathcal{T}_{\text {con,A }}^{s}$ relative to the $S^{3}$ compactification scale $\mu$. Here, we can take the limit $\mu / \Lambda \rightarrow \infty$, in which case the Klebanov-Tseytlin black holes approach conformal black holes in $A d S_{5} \times T^{1,1}$, see section 4.2. For the latter, the Hawking-Page transition temperature $T_{c}^{\text {conformal }}$ is given by (4.31), represented by the horizontal black line.

\footnotetext{
${ }^{19}$ This reflects the $\chi \mathrm{SB}$ phase transition between $\mathcal{V}_{A}^{s}$ and $\mathcal{V}_{B}$ vacua, see figure 5.
} 


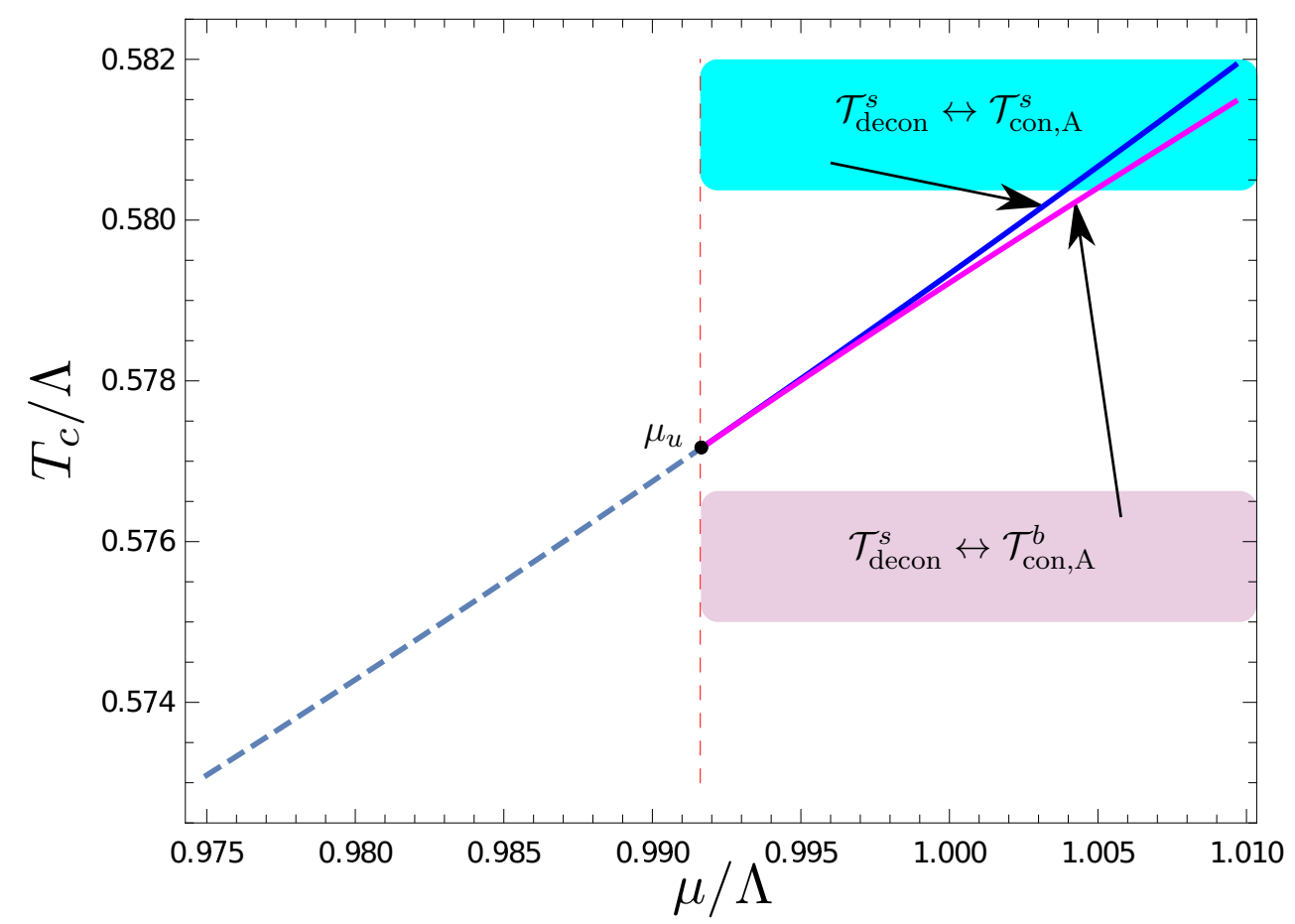

Figure 23. The temperature $T_{c}$ of the confinement/deconfinement phase transitions between the different states in the cascading gauge theory. Magenta curve represents the analog of the HawkingPage phase transition between the Klebanov-Tseytlin black holes and the $\mathcal{V}_{A}^{b}$ Euclidean vacua of the conifold flux geometries. The deconfined states are the preferred ones for $T>T_{c}$.

In figure 25, corresponding to the confinement/deconfinement temperatures $T_{c}$ we present the reduced energy densities $\hat{\mathcal{E}}_{c}$ (see (5.1)) of $\mathcal{T}_{\text {decon }}^{s}$ states, i.e., the KlebanovTseytlin black holes, at the transition point. Once again, the green curve represents $\mathcal{T}_{\text {decon }}^{s} \leftrightarrow \mathcal{T}_{\text {con, B }}$ transition, and the blue curves represents $\mathcal{T}_{\text {decon }}^{s} \leftrightarrow \mathcal{T}_{\text {con, } \mathrm{A}}^{s}$ transition. The deconfined states are the preferred ones above the corresponding $\hat{\mathcal{E}}_{c}$. The dashed curve indicates when the end-point of the phase transition, i.e., the $\mathcal{T}_{\text {decon }}^{s}$ states, are perturbatively unstable to chiral symmetry breaking. The red curve indicates the critical energy $\hat{\mathcal{E}}_{\chi \mathrm{SB}}$ : the Klebanov-Tseytlin black holes are unstable to chiral symmetry breaking for $\hat{\mathcal{E}}<\hat{\mathcal{E}}_{\chi \mathrm{SB}}$. The Klebanov-Strassler black holes exist only at energy densities $\hat{\mathcal{E}}<\hat{\mathcal{E}}_{\chi \mathrm{SB}}$, i.e., below the red curve. Note that the red curve $\hat{\mathcal{E}}_{\chi \mathrm{SB}}$ terminates at $\mu=\mu_{K S}$,

$$
\mu_{K S}=0.8766(6) \Lambda
$$

- there are no Klebanov-Strassler black holes for $\mu>\mu_{K S}$. We explain origin of $\mu_{K S}$ in section 6.3.2.

In figure 26 we present the reduced energy densities $\hat{\mathcal{E}}_{c}$ of $T_{\text {decon }}^{s}$ states at $\hat{\mathcal{E}}_{c}\left[\mathcal{T}_{\text {decon }}^{s} \leftrightarrow\right.$ $\mathcal{T}_{\text {con,A }}^{s}$ confinement/deconfinement transition (blue curve) and $\hat{\mathcal{E}}_{c}\left[\mathcal{T}_{\text {decon }}^{s} \leftrightarrow \mathcal{T}_{\text {con, A }}^{b}\right.$ confinement/deconfinement transition (magenta curve). These transitions are never realized in practice since the transition to a the preferred confined phase $\mathcal{T}_{\text {con,B }}$ occurs at higher energy densities (compare with figure 25). 


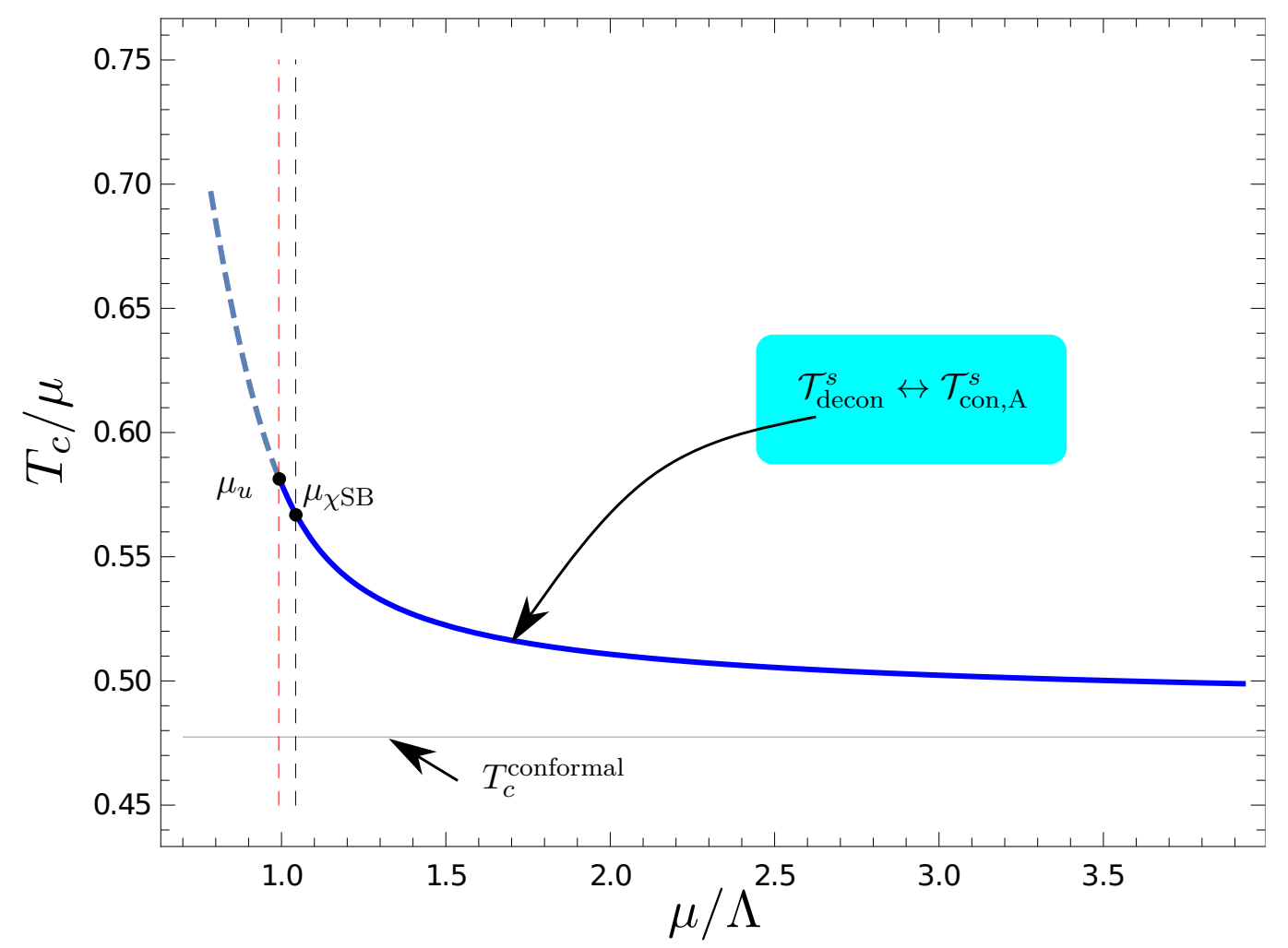

Figure 24. The temperature $T_{c}$ of the confinement/deconfinement phase transition $\mathcal{T}_{\text {decon }}^{s} \leftrightarrow \mathcal{T}_{\text {con, A }}^{s}$ relative to $S^{3}$ compactification scale $\mu$. For large values of $\mu / \Lambda$ it approaches its conformal limit $T_{c}^{\text {conformal }}$, given by (4.31). The deconfined states are the preferred ones for $T>T_{c}$.

Recall that $T_{u}(\mu)$ is the terminal temperature for $\mathcal{T}_{\text {decon }}^{s}$ states - the Klebanov-Tseytlin black holes. Thus $T_{\chi \mathrm{SB}}(\mu)$, the critical temperature at the onset of the chiral symmetry breaking, can not exceed $T_{u}(\mu)$. In the left panel of figure 27 we present results for $T_{\chi \mathrm{SB}}$. $\mu^{\star}$, see (6.15), identifies the $S^{3}$ compactification scale when both critical temperatures $T_{u}$ and $T_{\chi \mathrm{SB}}$ coincide. In the right panel of figure 27 we explain how to compute $\mu^{\star}$ : we evaluate the reduced specific heat density $\hat{c}_{V}(6.6)$ along the $\hat{\mathcal{E}}_{\chi \mathrm{SB}}$ curve for $\mathcal{T}_{\text {decon }}^{s}$ states and identify the value of $\mu$ when it diverges.

Much like for $\mu=0$, for the values of $\mu$ when both $\mathcal{T}_{\text {decon }}^{s, \pm}$ and $\mathcal{T}_{\text {decon }}^{b}$ states exist, the free energy density of $\mathcal{T}_{\text {decon }}^{s,+}$ states is always lower than that of $\mathcal{T}_{\text {decon }}^{b}$ states, ${ }^{20}$ i.e., whenever both Klebanov-Strassler and Klebanov-Tseytlin black holes exist, the latter ones (on the branch with the positive specific heat) always dominate in the canonical ensemble. ${ }^{21}$ This also implies that even if theoretically possible, the confinement/deconfinement phase transitions $\mathcal{T}_{\text {decon }}^{b} \leftrightarrow \mathcal{T}_{\text {con, B }}$, i.e., between the Klebanov-Strassler black holes and the $\mathcal{V}_{B}$ Euclidean vacua would never occur in practice: these phase transitions would always be preceded by $\mathcal{T}_{\text {decon }}^{s,+} \leftrightarrow \mathcal{T}_{\text {con,B }}$ phase transitions.

\footnotetext{
${ }^{20}$ See section 6.2 .3 for some examples.

${ }^{21}$ As we will see in section 6.2.2, the story in the microcanonical ensemble is different: whenever both exist, Klebanov-Strassler black holes are always more entropic at a fixed energy density than the KlebanovTseytlin black holes.
} 


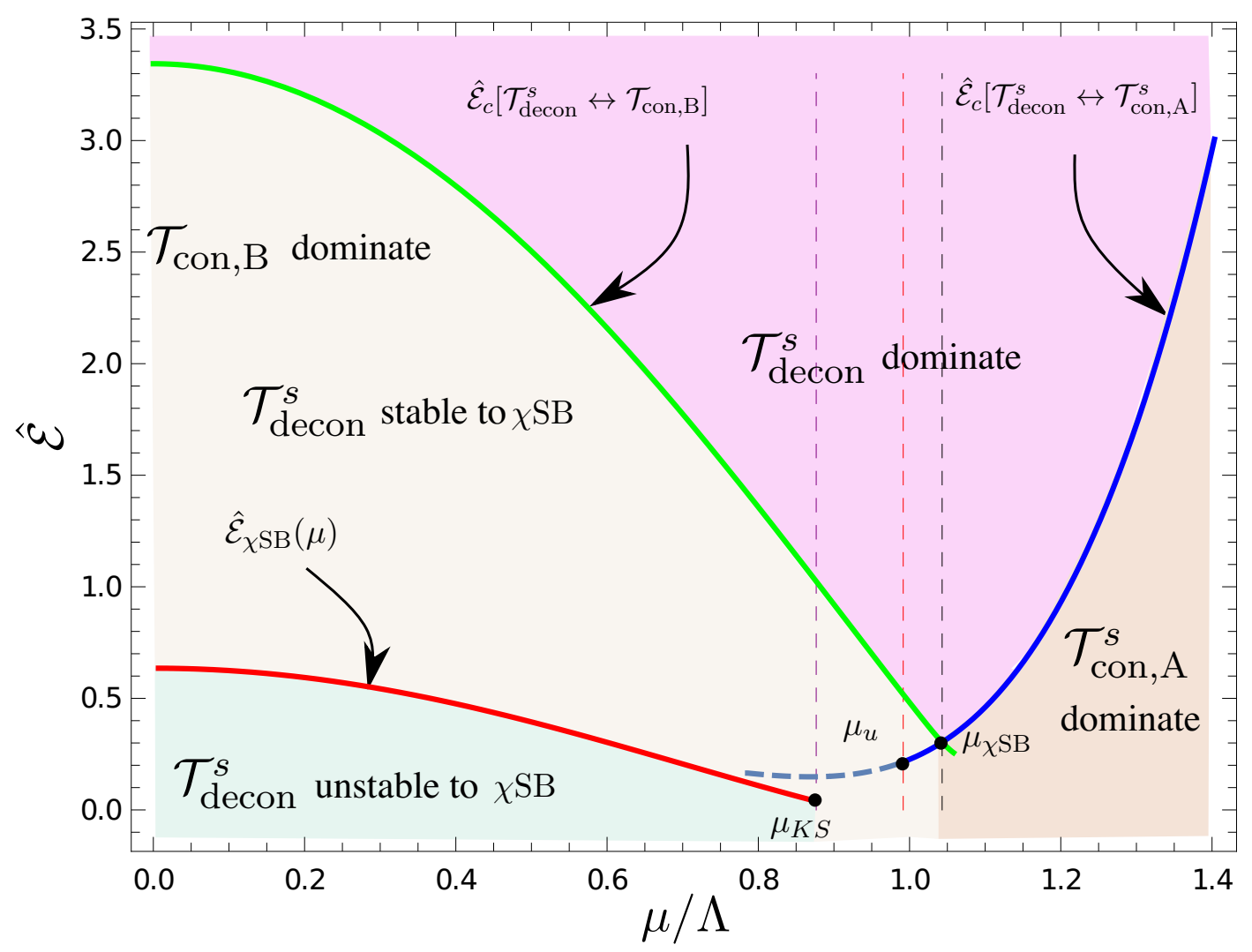

Figure 25. Critical energies $\hat{\mathcal{E}}_{c}$ of $\mathcal{T}_{\text {decon }}^{s}$ states (Klebanov-Tseytlin black holes) for different confinement/deconfinement phase transitions. The deconfined states are preferred above green and blue curves. The Klebanov-Tseytlin black holes become unstable to $\chi \mathrm{SB}$ fluctuations below the red curve $\hat{\mathcal{E}}_{\chi \mathrm{SB}}(\mu)$. The Klebanov-Strassler black holes exist only for $\hat{\mathcal{E}}<\hat{\mathcal{E}}_{\chi \mathrm{SB}}$ and only when $\mu<\mu_{K S}(6.17)$.

Some of the potential confinement/deconfinement phase transitions, are never realized (even theoretically):

- $\mathcal{T}_{\text {decon }}^{b} \leftrightarrow \mathcal{T}_{\text {con,A }}^{s}$ - the transition between the Klebanov-Strassler black holes and the $\mathcal{V}_{A}^{s}$ Euclidean vacua. These transitions do not exist because there are no KlebanovStrassler black holes for $\mu>\mu_{u}$, and there are no stable $\mathcal{V}_{A}^{s}$ vacua (correspondingly $\mathcal{T}_{\text {con }, \mathrm{A}}^{s}$ confined thermal states) for $\mu<\mu_{u}$, see figures 25 and 5 .

- $\mathcal{T}_{\text {decon }}^{b} \leftrightarrow \mathcal{T}_{\text {con,A }}^{b}$ - the transition between the Klebanov-Strassler black holes and the $\mathcal{V}_{A}^{b}$ Euclidean vacua. These transitions do not exist because there are no KlebanovStrassler black holes for $\mu>\mu_{u}$, and there are $\mathcal{V}_{A}^{b}$ vacua (correspondingly $\mathcal{T}_{\text {con,A }}^{b}$ confined thermal states) for $\mu<\mu_{u}$, see figures 25 and 6 .

\subsubsection{Microcanonical ensemble}

The phase diagram in the microcanonical ensemble for the conifold black branes $(\mu=0)$ morally repeats for black holes at $\mu / \Lambda>0$ : 


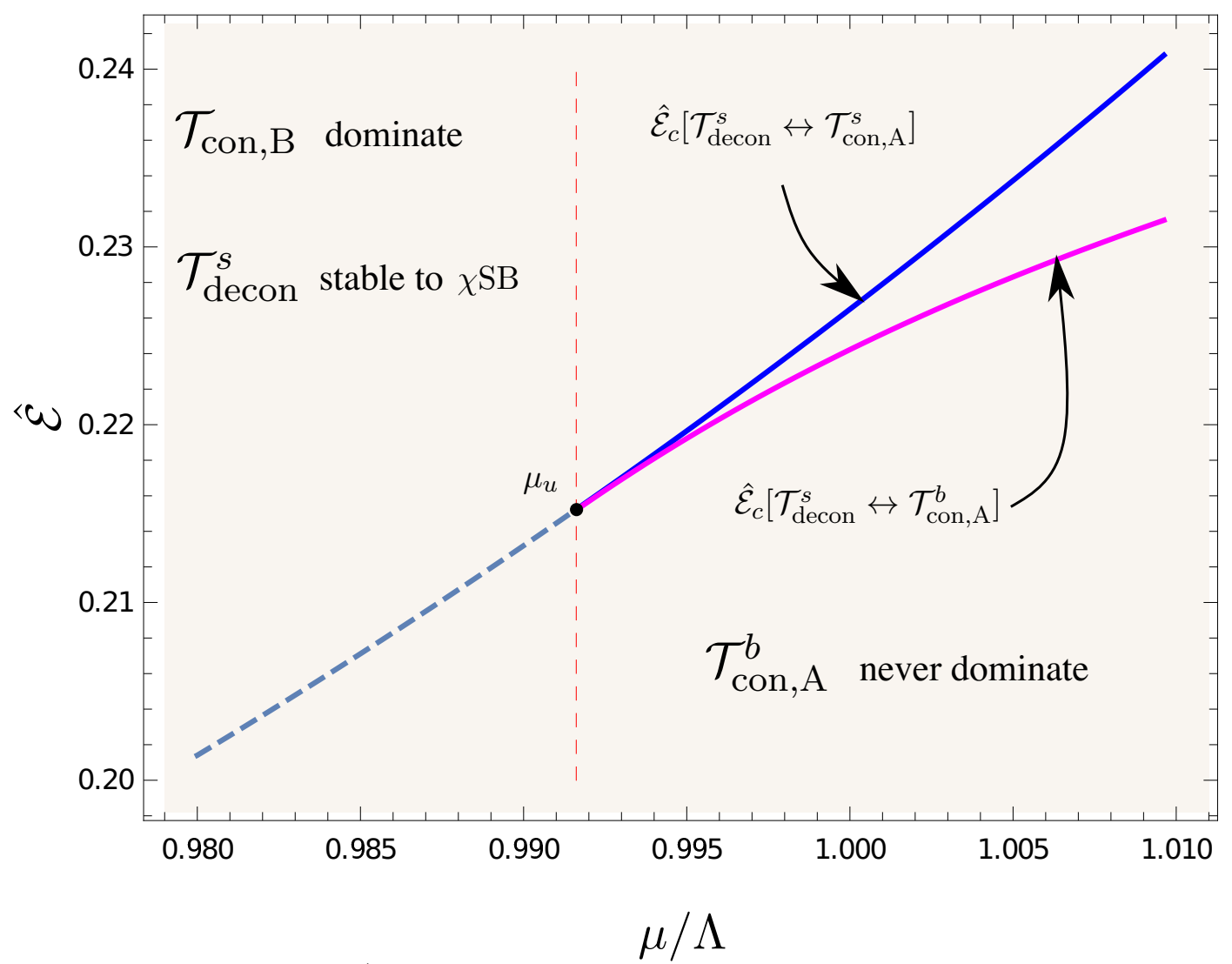

Figure 26. Critical energies $\hat{\mathcal{E}}_{c}$ of $\mathcal{T}_{\text {decon }}^{s}$ states (Klebanov-Tseytlin black holes) for different confinement/deconfinement phase transitions. These transitions do not happen in practice since the transition to a dominant confined phase $\mathcal{T}_{\text {con,B }}$ occurs at higher energy densities (compare with figure 25).
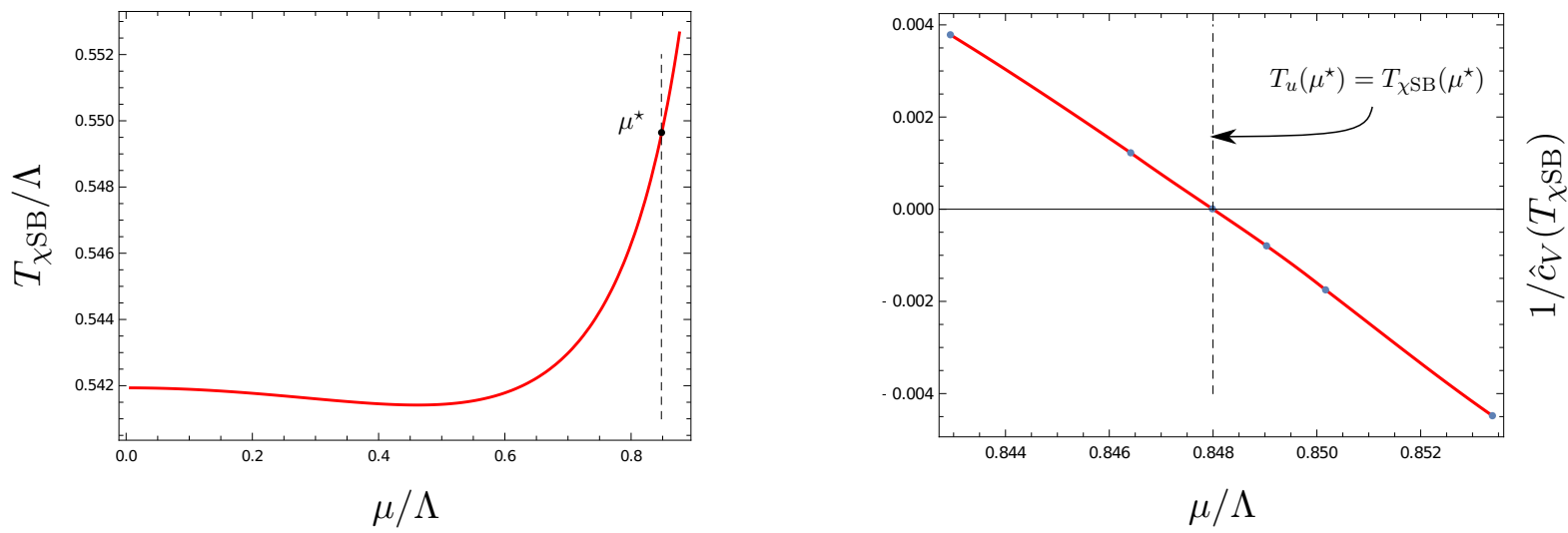

Figure 27. Left panel: the critical temperature $T_{\chi \mathrm{SB}}$ of the spontaneous chiral symmetry breaking in $\mathcal{T}_{\text {decon }}^{s}$ states, i.e., the Klebanov-Tseytlin black holes, as a function of $\mu / \Lambda$. Right panel: $\mu^{*}$, see (6.15), identifies the $S^{3}$ compactification scale $\mu$ with the divergent reduced specific heat $\hat{c}_{V}$ at $T_{\chi \mathrm{SB}}\left(\mu^{*}\right)$. At $\mu=\mu^{\star}$ the critical temperature $T_{\chi \mathrm{SB}}$ coincides with the terminal temperature $T_{u}$ of $\mathcal{T}_{\text {decon }}^{s}$ states. 


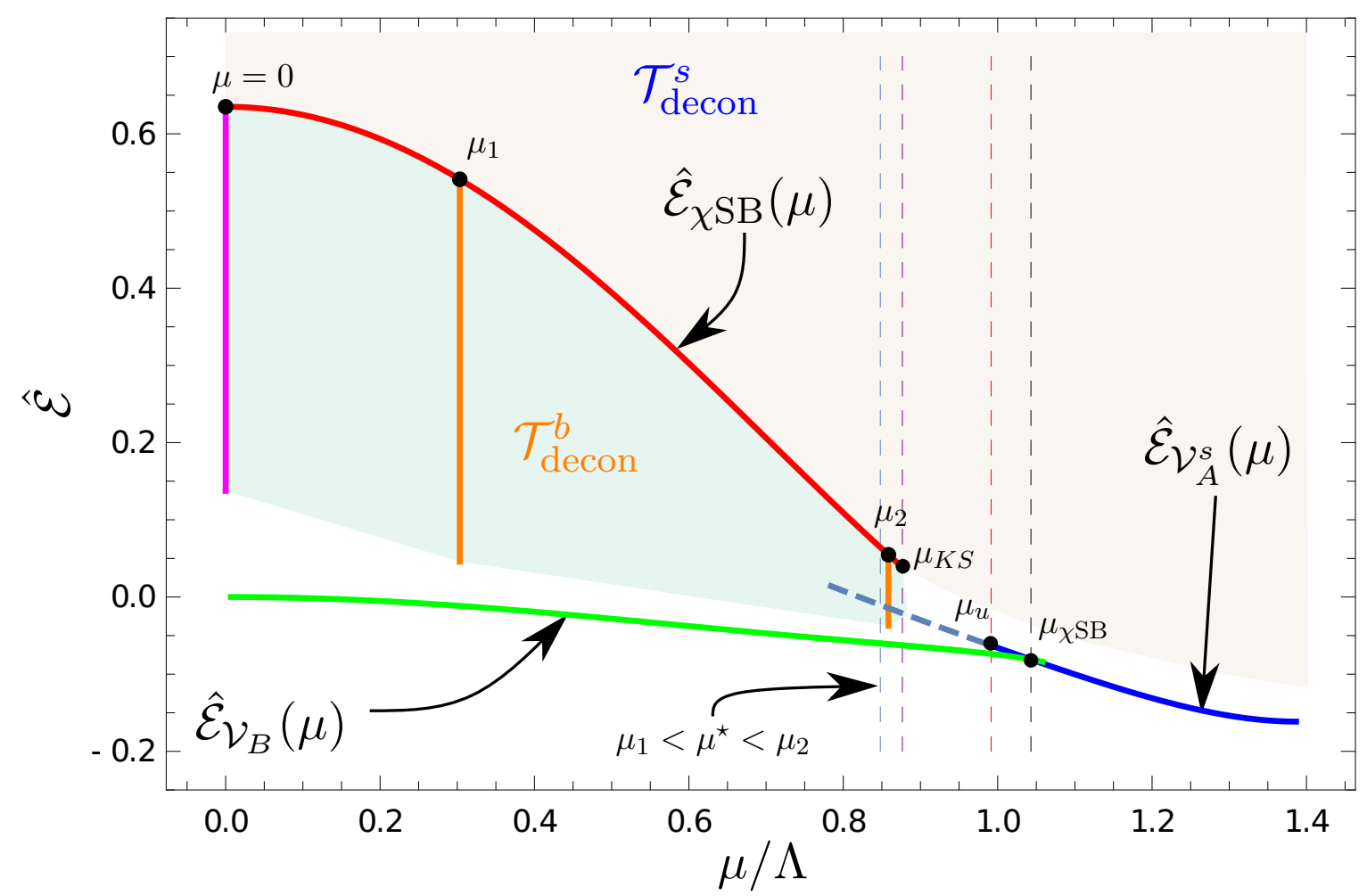

Figure 28. Landscape of the Klebanov-Tseytlin black holes, $\mathcal{T}_{\text {decon }}^{s}$ states, (the shaded region labeled by $\mathcal{T}_{\text {decon }}^{s}$ ) and the Klebanov-Strassler black holes, $\mathcal{T}_{\text {decon }}^{b}$ states, (the shaded region labeled by $\left.\mathcal{T}_{\text {decon }}^{b}\right)$. Two $\mathcal{T}_{\text {decon }}^{b}$ state spectra at $\mu=\mu_{1}$ and $\mu=\mu_{2}$ are represented by the vertical orange lines. The vertical magenta line represents the spectrum of the Klebanov-Strassler black branes, $\mathcal{T}_{\text {decon }}^{s}$ states at $\mu=0$.

- Confined states have vanishing reduced entropy density $\hat{s}$ (6.1) in the large $M$ limit (the supergravity approximation of the dual geometry), and thus are never the preferred states if any of the deconfined states $\mathcal{T}_{\text {decon }}^{s}$, i.e., the Klebanov-Tseytlin, or $\mathcal{T}_{\text {decon }}^{b}$, i.e., the Klebanov-Strassler black holes, exist.

- When both the $\mathcal{T}_{\text {decon }}^{s}$ and $\mathcal{T}_{\text {decon }}^{b}$ states exist, the latter ones are always more entropic, and thus represent the preferred states.

Since $\mathcal{T}_{\text {decon }}^{b}$ states at $\mu=0$ have the negative specific heat, the corresponding Klebanov-Strassler black branes are perturbatively unstable to metric fluctuations breaking the translational invariance of the horizon [41]. This link is no longer true for the black holes, and the unstable hydrodynamic sound mode of the black brane extended horizon could be lifted by the compactification [43].

In figure 28 we discuss the landscape of $\mathcal{T}_{\text {decon }}^{s}$ and $\mathcal{T}_{\text {decon }}^{b}$ states. For reference, the green and the blue curves represent the vacua states $\mathcal{V}_{B}$ and $\mathcal{V}_{A}^{s}$ correspondingly. The red curves reproduces the critical energy density $\hat{\mathcal{E}}_{\chi \mathrm{SB}}$ for the range $\mu \in\left[0, \mu_{K S}\right] . \mu_{K S}$ is the terminal point of the red curve. The Klebanov-Tseytlin black holes are the preferred states in the microcanonical ensemble in the shaded region above the red the blue curves, labeled 
by $\mathcal{T}_{\text {decon }}^{s}$. The Klebanov-Strassler black holes are the preferred states in the shaded region labeled by $\mathcal{T}_{\text {decon }}^{b}$. They exist below the red curve, and only for $\mu<\mu_{K S}$ (6.17). The vertical magenta line, at $\mu=0$, represents the Klebanov-Strassler black brane constructed in [4]. It extends along the $\hat{\mathcal{E}}$ axis indicative to which reduced energy density, as the lower limit, we have been able to construct this black brane numerically. Note that there is likely a 'gap' between the magenta curve and the green curve representing the vacuum $\mathcal{V}_{B}$. Likewise, the sample of two vertical orange curves at $\mu=\mu_{1}$ and $\mu=\mu_{2}$ represents the energy spectra of the Klebanov-Strassler black holes, see section 6.2.3 for additional details. Once again, notice the potential gap in the spectrum of the Klebanov-Strassler black holes and the appropriate vacuum states $\mathcal{V}_{B}$. There is also a potential gap in the spectrum of Klebanov-Tseytlin black holes and the appropriate vacuum states $\mathcal{V}_{A}^{s}$. We emphasize this gap since it points out that the non-equilibrium energy states on $S^{3}$-compactified conifold geometries with fluxes, alternatively the low energy density states of the cascading gauge theory, might not thermalize along $\mathrm{SO}(4)$-invariant evolution trajectories. ${ }^{22}$ Note that for our sample scales, $\mu_{1}<\mu^{\star}<\mu_{2}$ where $\mu_{\star}$ (6.15) affects the sequence of the various phase transitions, see 6.2 .3 for further details. Recall that $\mathcal{V}_{A}^{s}$ vacua are perturbatively unstable to the spontaneous chiral symmetry breaking when $\mu<\mu_{u}$, represented by the dashed curve. Interestingly, the Klebanov-Strassler black holes at $\mu=\mu_{2}$ reaches the energy densities of some of these unstable states - the corresponding vertical orange line intersects the dashed line of the unstable $\mathcal{V}_{A}^{s}$ vacua. Thus, at least when $\mu<\mu_{K S}$, the unstable to $\chi \mathrm{SB}$ fluctuations $\mathcal{V}_{A}^{s}$ vacua are expected to thermalize as Klebanov-Strassler black holes. It is very interesting to understand the fate of the unstable $\mathcal{V}_{A}^{s}$ vacua for $\mu \in\left(\mu_{K S}, \mu_{u}\right)$ - we do not have the answer to this question.

\subsubsection{Black holes thermodynamics for select values of $\mu / \Lambda \neq 0$}

In this section we follow the discussion of section 6.1 and presents results for the conifold black holes thermodynamics at

$$
\mu_{1}=0.303(3) \Lambda \quad \text { and } \quad \mu_{2}=0.858(5) \Lambda .
$$

Note that $\mu_{1}<\mu^{\star}(6.15)$ and $\mu_{2}>\mu^{\star}$ - as we will see the relation of the compactification scale $\mu$ relative to $\mu^{\star}$ affects the sequences of the phase transitions for the $\mathcal{T}_{\text {decon }}^{s}$ states.

At $\mu=\mu_{1}$ there is a single confinement-deconfinement phase transition:

$$
\mathcal{T}_{\text {decon }}^{S} \leftrightarrow \mathcal{T}_{\text {con }, \mathrm{B}}: \quad T_{c}\left(\mu_{1}\right)=0.612(1) \Lambda, \quad \hat{\mathcal{E}}_{c}\left(\mu_{1}\right)=3.025(2) .
$$

At $\mu=\mu_{2}$, theoretically there are two confinement-deconfinement phase transition:

$$
\begin{array}{lll}
\mathcal{T}_{\text {decon }}^{s} \leftrightarrow \mathcal{T}_{\text {con }, \mathrm{B}}: & T_{c}\left(\mu_{2}\right)=0.596(9) \Lambda, & \hat{\mathcal{E}}_{c}\left(\mu_{2}\right)=1.103(5), \\
\mathcal{T}_{\text {decon }}^{s} \leftrightarrow \mathcal{T}_{\text {con }, \mathrm{A}}^{s}: & T_{c}\left(\mu_{2}\right)=0.553(4) \Lambda, & \hat{\mathcal{E}}_{c}\left(\mu_{2}\right)=0.148(8) .
\end{array}
$$

In practice only the transition at higher temperature/energy density occurs. All these transitions are of the first order, with the deconfined phase being the preferred state at $T>T_{c}$ or $\hat{\mathcal{E}}>\hat{\mathcal{E}}_{c}$.

\footnotetext{
${ }^{22}$ See further discussion of this in holography in [44, 45].
} 

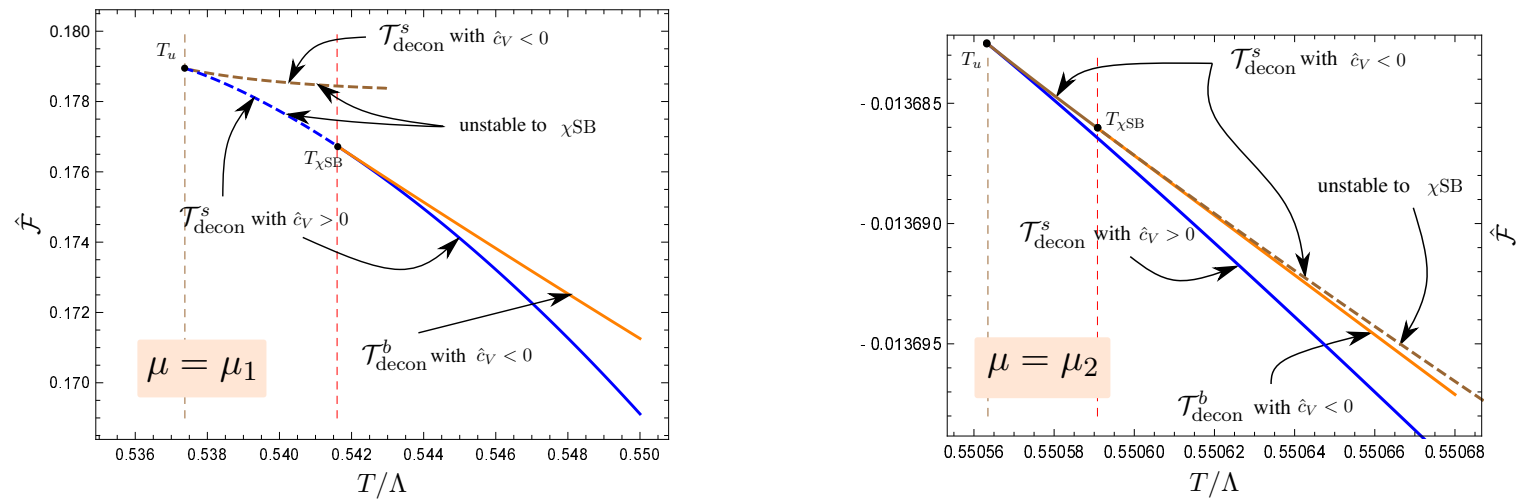

Figure 29. Typical phase diagram in the canonical ensemble for deconfined states $\mathcal{T}_{\text {decon }}^{s}$ (the Klebanov-Tseytlin black holes) and $\mathcal{T}_{\text {decon }}^{b}$ (the Klebanov-Strassler black holes) for $0<\mu=\mu_{1}<\mu^{\star}$ (see (6.15)) (the left panel), and for $\mu=\mu_{2}>\mu^{\star}$ (the right panel).

In figure 29 we highlight the similarities and the differences between the phase diagrams at $\mu=\mu_{1}$ (the left panel) and $\mu=\mu_{2}$ (the right panel) in the canonical ensemble. $T_{u}$ denotes the terminal point of $\mathcal{T}_{\text {decon }}^{s}$ states, i.e., the Klebanov-Tseytlin black holes; $T_{\chi \mathrm{SB}}$ is the critical temperature for the onset of the perturbative $\chi \mathrm{SB}$ instability of $\mathcal{T}_{\text {decon }}^{s}$ states.

$T_{u}$ is a bifurcation point of the two branches of $\mathcal{T}_{\text {decon }}^{s}$ states: with the positive specific heat $\mathcal{T}_{\text {decon }}^{s,+}$ (blue), and the negative specific heat $\mathcal{T}_{\text {decon }}^{s,-}$ (brown). The critical behavior as $T \rightarrow T_{u}$ is exactly the same as for $\mu=0$, see (6.8).

While at $\mu=\mu_{1} \mathcal{T}_{\text {decon }}^{s,+}$ states are unstable to $\chi \mathrm{SB}$ for $T<T_{\chi \mathrm{SB}}$ (blue dashed curve), at $\mu=\mu_{2}$ it is the $\mathcal{T}_{\text {decon }}^{s,-}$ states that are unstable to $\chi \mathrm{SB}$ for $T>T_{\chi \mathrm{SB}}$ (brown dashed curve). The critical behavior as $T \rightarrow T_{\chi \mathrm{SB}}$ is exactly the same as for $\mu=0$, see (6.9).

$\mathcal{T}_{\text {decon }}^{b}$ states (orange curves), i.e., the Klebanov-Strassler black holes, exist for $T>$ $T_{\chi \mathrm{SB}}$. These black holes always have a negative specific heat. Although they are thermodynamically unstable, they are not necessarily dynamically unstable [41, 43].

When multiple phases present, $\mathcal{T}_{\text {decon }}^{s,+}$ is always the preferred one:

$$
\hat{\mathcal{F}}\left[\mathcal{T}_{\text {decon }}^{s,+}\right]<\hat{\mathcal{F}}\left[\mathcal{T}_{\text {decon }}^{b}\right]<\hat{\mathcal{F}}\left[\mathcal{T}_{\text {decon }}^{s,-}\right]
$$

The order of the transition from the $\mathcal{T}_{\text {decon }}^{b}$ states to (dominant) $\mathcal{T}_{\text {decon }}^{s,+}$ states differs: it is of the second order at $\mu=\mu_{1}$ and of the first-order at $\mu=\mu_{2}$.

The phase transition from the $\mathcal{T}_{\text {decon }}^{s,-}$ states to (dominant) $\mathcal{T}_{\text {decon }}^{s,+}$ states is always of the first order.

In figure 30 we present the typical phase diagram in the microcanonical ensemble for the black holes on the conifold.

$\mathcal{T}_{\text {decon }}^{b}$ (the orange curves; the Klebanov-Strassler black holes) are always the preferred states, when they exist along with $\mathcal{T}_{\text {decon }}^{s}$ (blue/brown curves, the Klebanov-Tseytlin black holes) states.

$\mathcal{T}_{\text {decon }}^{b}$ states exist for $\hat{\mathcal{E}}<\hat{\mathcal{E}}_{\chi \mathrm{SB}}$, in which regime the Klebanov-Tseytlin black holes are perturbatively unstable to $\chi \mathrm{SB}$ fluctuations (dashed curves).

Note that $\hat{\mathcal{E}}_{\chi \mathrm{SB}}>\hat{\mathcal{E}}_{u}$ at $0<\mu=\mu_{1}<\mu^{\star}$, but $\hat{\mathcal{E}}_{\chi \mathrm{SB}}<\hat{\mathcal{E}}_{u}$ at $\mu=\mu_{2}>\mu^{\star}$. 

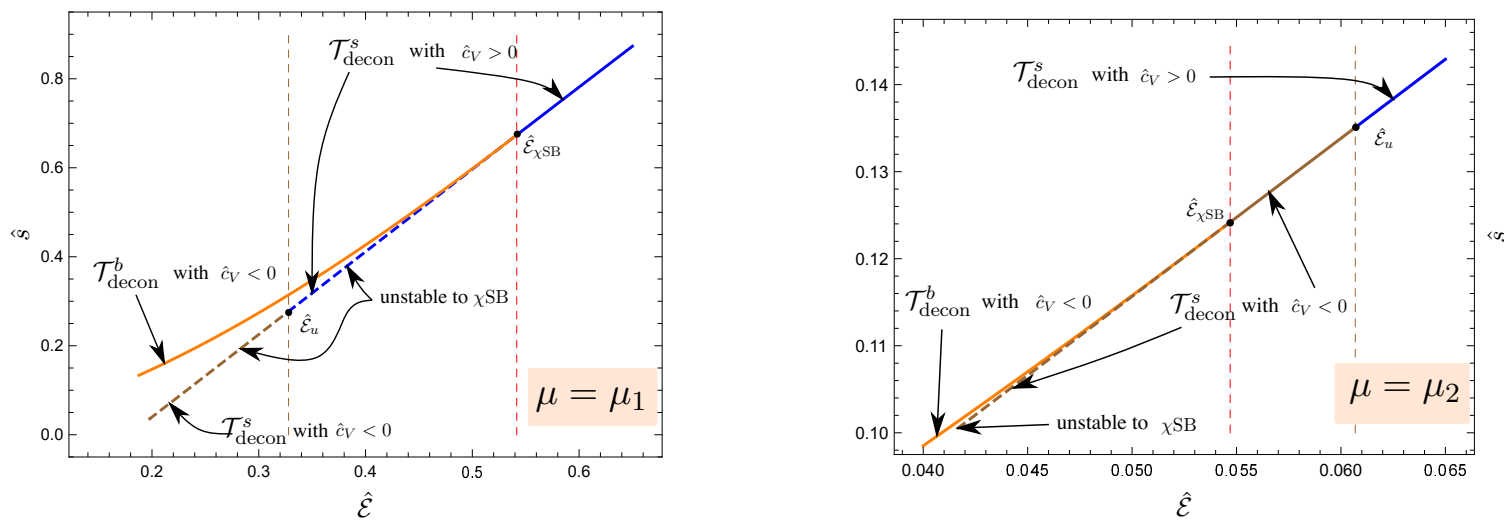

Figure 30. Typical phase diagram in the microcanonical ensemble for deconfined states $\mathcal{T}_{\text {decon }}^{s}$ (the Klebanov-Tseytlin black holes) and $\mathcal{T}_{\text {decon }}^{b}$ (the Klebanov-Strassler black holes) for $0<\mu=\mu_{1}<\mu^{\star}$ (see (6.15)) (the left panel), and for $\mu=\mu_{2}>\mu^{\star}$ (the right panel).

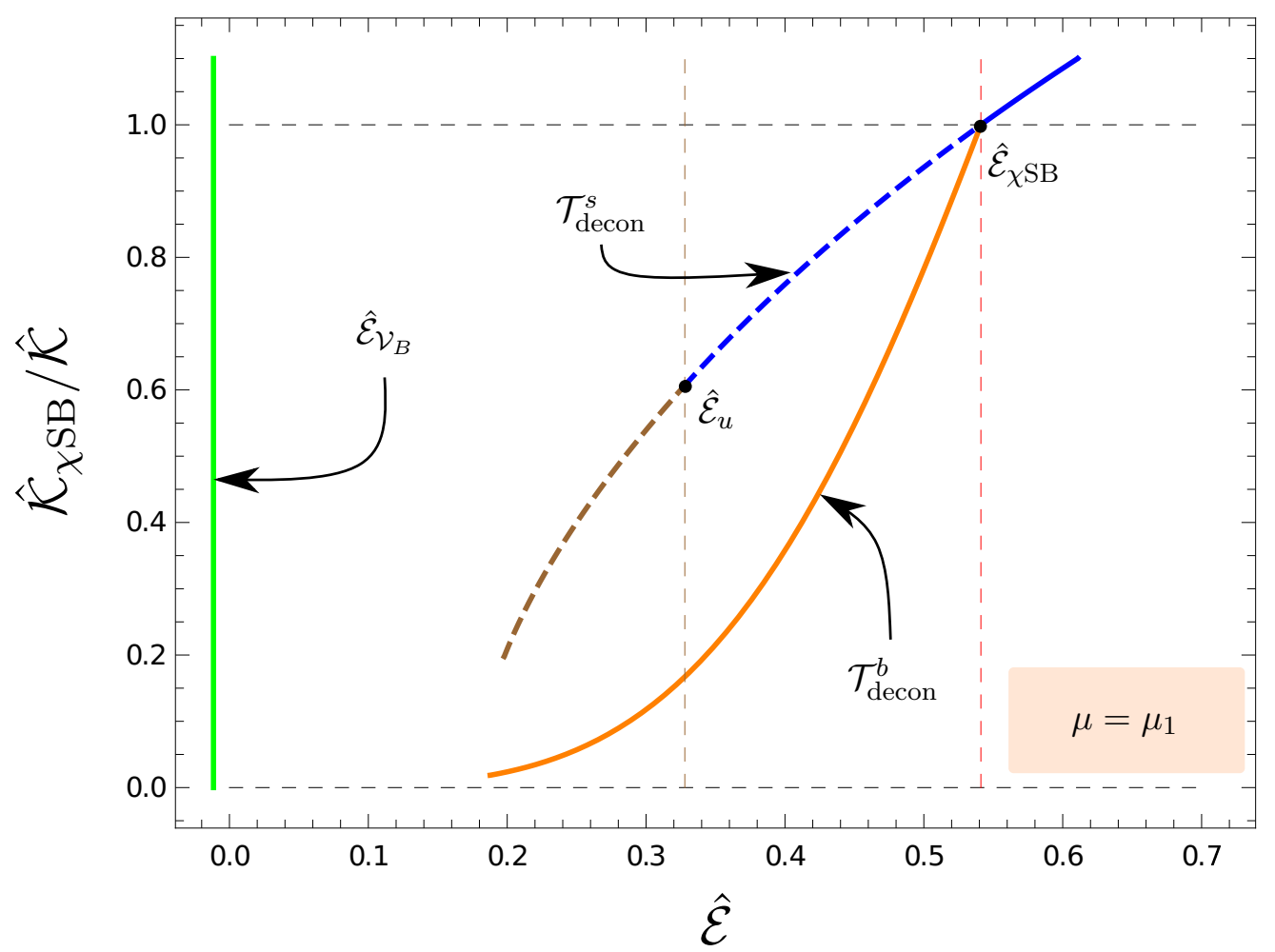

Figure 31. Inverse Kretschmann scalar $\hat{\mathcal{K}}$ normalized to its value at $\hat{\mathcal{E}}=\hat{\mathcal{E}}_{\chi \mathrm{SB}}$ for $\mathcal{T}_{\text {decon }}^{s}$ states (blue/drown curves) and $\mathcal{T}_{\text {decon }}^{b}$ states (orange curve) at $\mu=\mu_{1}$. Vertical green line denotes the energy density of the vacuum $\hat{\mathcal{V}}_{B}$ at $\mu=\mu_{1}$. 
In figure 31 we present the inverse of the reduced Kretschmann scalar $\hat{\mathcal{K}}$ (5.7), normalized to its value at the $\hat{\mathcal{E}}=\hat{\mathcal{E}}_{\chi \mathrm{SB}}$ for $\mathcal{T}_{\text {decon }}^{s}$ states (blue/drown curves, the Klebanov-Tseytlin black holes) and $\mathcal{T}_{\text {decon }}^{b}$ states (orange curve, the Klebanov-Strassler black holes) at $\mu=\mu_{1}$. The vertical green line indicates the energy density of the $\hat{\mathcal{V}}_{B}$ vacuum state at $\mu=\mu_{1}$. Our numerics suggests ${ }^{23}$ that $\hat{\mathcal{K}}$ likely diverges in deconfined states prior to reaching the vacuum energy - there is potentially a gap in the spectrum of black holes on the conifold.

\subsection{Technical details on constructing the black holes, computing $T_{\chi \mathrm{SB}}(\mu)$ and $T_{c}(\mu)$}

In constructing black holes on the conifold with fluxes it is convenient to start at $\mu=0$ and slowly increase the $S^{3}$ curvature, compare to the strong coupling scale $\Lambda$ of the theory. At $\mu=0$ we have black branes, thus we would like to recycle the numerical results obtained in $[3,4,11]$. In the latter references the "universal" radial coordinate $x$,

$$
1-x \equiv-\frac{G_{t t}}{G_{x^{i} x^{i}}}, \quad x \in[0,1]
$$

was used, with $x \rightarrow 0_{+}$being the asymptotic boundary, and $x \rightarrow 1_{-}$being the regular Schwarzschild horizon. It would appear to be more natural to use the same radial coordinate $x$, instead of $\rho$ as defined in (3.15) and (3.19). The problem is that the radial coordinate $x$ is simply not defined when $\mu \neq 0$. Indeed, the definition (6.22) is sensible only that the ratio of the warp factors $G_{t t}$ and $G_{x^{i} x^{i}}$ is a monotonic function from the boundary to the horizon. When $\mu \neq 0$, this function is not monotonic. Indeed, even in the conformal limit, see (4.22),

$$
-\frac{G_{t t}}{G_{x^{i} x^{i}}}=\frac{4\left(f_{a, 1,0} \rho+1\right)\left(K_{0} \mu^{2} \rho^{2}+2 f_{a, 1,0}^{2} \rho^{2}+4 f_{a, 1,0} \rho+4\right)}{\left(f_{a, 1,0} \rho+2\right)^{4}},
$$

has a local maximum at

$$
\rho=\rho_{\max } \equiv \frac{2 \mu\left(\left(2 \kappa^{2}+2 \mu^{2}\right)^{1 / 2}+\mu\right)}{\left(2 \kappa^{2}+\mu^{2}\right) K_{0}^{1 / 2} \kappa},
$$

with $\kappa$ defined in as in (4.25), and

$$
-\left.\frac{G_{t t}}{G_{x^{i} x^{i}}}\right|_{\rho=0}=1, \quad-\left.\frac{G_{t t}}{G_{x^{i} x^{i}}}\right|_{\rho=\rho_{\max }}>1, \quad-\left.\frac{G_{t t}}{G_{x^{i} x^{i}}}\right|_{\rho \rightarrow+\infty}=0 .
$$

Of course, at $\mu=0$, we can fully map the results for the black branes on the conifold in $x$ radial coordinate to those in $\rho$ : from eq. (A.11) and (A.17) of [4]

$$
\begin{aligned}
K_{1}^{[x]} & =P^{2} g_{0} k_{s}-\frac{1}{2} P^{2} g_{0} \ln x+\mathcal{O}\left(x^{3 / 4} \ln x\right), \quad g^{[x]}=g_{0}[1+\mathcal{O}(x \ln x)], \\
h^{[x]} & =\frac{P^{2} g_{0}}{a_{0}^{2}}\left(\frac{1}{8}+\frac{k_{s}}{4}\right)-\frac{P^{2} g_{0}}{8 a_{0}^{2}} \ln x+\mathcal{O}\left(x^{1 / 2} \ln x\right),
\end{aligned}
$$

\footnotetext{
${ }^{23} \mathrm{~A}$ definite conclusion requires more precise analysis with improved numerical codes.
} 
and using

$$
-\frac{G_{t t}}{G_{x^{i} x^{i}}} \equiv(1-x)^{2} \equiv f(\rho) \quad \Longrightarrow \quad x=1-\sqrt{f},
$$

with the asymptotic expansions (A.50), (A.53) and (A.54) we find

$$
g_{s}=g_{0}, \quad K_{0}=P^{2} g_{0}\left(k_{s}+\frac{1}{2} \ln 2-\frac{1}{2} \ln \left(-f_{4,0}\right)\right) .
$$

Additionally, matching the $g_{t t}$ components of the metric on $\mathcal{M}_{5}(3.2)$ in different radial coordinates,

$$
g_{t t} \equiv \frac{(1-x)^{2}}{\left(h^{[x]}\right)^{1 / 2}\left(2 x-x^{2}\right)^{1 / 2}} \equiv \frac{f}{h^{1 / 2} \rho^{2}} \quad \Longrightarrow \quad f_{4,0}=-a_{0}^{2},
$$

where $a_{0}^{2} \propto s T$ of the thermal states of the black branes on the conifold. The scaling symmetries of the equations of motion in $[3,4,11]$ were always used to set $a_{0}=1$, with varying $k_{s}$. This means, effectively, keeping the temperature scale 'fixed', while varying the strong coupling scale $\Lambda$ of the theory. The results were presented in terms of a single dimensionless ratio $\frac{T}{\Lambda}$. Setting $a_{0}=1$ implies that, see (6.29),

$$
f_{4,0}=-1
$$

While (6.30) was established for $\mu=0$, we keep it even for $\mu \neq 0$. This means that out of three scales $\mu, \Lambda, T$ relevant to the black holes on the conifold, we fixed one of them - the temperature. As in $[3,4,11]$, this is consistent as long as we use dimensionless observables.

We now outline the steps used to produced the data reported in section 6.2.

\subsubsection{Klebanov-Tseytlin black holes at $\mu \neq 0$}

In addition to (6.30), we use the scaling symmetries (3.24) and (3.25) to fix

$$
P=1, \quad g_{s}=1 .
$$

For the data files of [4] we identify the black brane solution corresponding to (see (2.48))

$$
K_{0}=4 \quad \Longleftrightarrow \quad \Lambda=2^{1 / 4} e^{-2},
$$

i.e., when $k_{s}=4-\ln 2 / 2$. We use the coordinate transformation $x \leftrightarrow \rho$ of (6.27) to map the corresponding black brane data to (A.55), (A.58), remembering the constraints of the unbroken chiral symmetry (A.61). We thus obtained our first entry line for the KlebanovTseytlin black hole data file at $\mu=0$. We populate this $K T$-master file, with each line labeled by $\mu$, keeping fixed $f_{4,0}, P, g_{s}, K_{0}$ as in (6.30)-(6.32). Each entry line in the file represents a black hole at a single value of $T / \Lambda$, labeled by

$$
\frac{\mu}{\Lambda}=2^{-1 / 4} e^{2} \mu \equiv \hat{\mu} \text {. }
$$

Given this KT-master file, we can produce Klebanov-Tseytlin black holes at fixed $\mu / \Lambda$, for different values of $T / \Lambda$ as follows: ${ }^{24}$ pick an entry from the KT-master file at required value of $\hat{\mu}_{r}$. This is a Klebanov-Tseytlin black holes at some temperature $T$, see (A.59).

\footnotetext{
${ }^{24}$ We use a separate file for distinct values of $\mu / \Lambda$.
} 
We continue to keep (6.30) and (6.31), but we now vary both $K_{0}$ and $\mu$ along the line

$$
\left\{K_{0}, \mu\right\} \equiv\left\{K_{0}, \hat{\mu}_{r} 2^{1 / 4} e^{-K_{0} / 2}\right\} .
$$

Since we vary $K_{0}$, we vary the strong coupling scale of the theory

$$
\Lambda=2^{1 / 4} e^{-K_{0} / 2},
$$

and thus, the ratio $T / \Lambda$. The corresponding change in $\mu$ as per (6.34) guarantees that during this $T / \Lambda$ variation

$$
\frac{\mu}{\Lambda}=\hat{\mu}_{r} 2^{1 / 4} e^{-K_{0} / 2} \times 2^{-1 / 4} e^{K_{0} / 2}=\hat{\mu}_{r}=\text { constant } .
$$

Each Klebanov-Tseytlin black hole data file (at a given value of $\hat{\mu}_{r}$ ) is verified with respect to the first law of thermodynamics (A.91). For example, the left panel of figure 35 verifies this for the Klebanov-Tseytlin black hole at $\mu=\mu_{1}(6.18)$ - the accuracy is $\sim 10^{-7}$ and better. This is a typical accuracy achieved for the Klebanov-Tseytlin black holes at $\mu / \Lambda \neq 0$.

\subsubsection{Computation of $T_{\chi \mathrm{SB}}(\mu)$}

$T_{\chi \mathrm{SB}}$ is the temperature of the onset of the perturbative chiral symmetry breaking instability of $\mathcal{T}_{\text {decon }}^{s}$ states, i.e., the Klebanov-Tseytlin black holes. To identify it we study $\chi \mathrm{SB}$ fluctuations about $\mathcal{T}_{\text {decon }}^{s}$ states and search for the normalizable mode.

As in (5.32), we set

$$
f_{a} \equiv f_{3}+\delta f, f_{b} \equiv f_{3}-\delta f, K_{1} \equiv K+\delta k_{1}, K_{3} \equiv K-\delta k_{1}, K_{2} \equiv 1+\delta k_{2},
$$

with the remaining metric functions and the string coupling as in $\mathcal{T}_{\text {decon }}^{s}$ thermal states $\left\{f, f_{c}, h, g\right\}$. It is straightforward to verify that truncation to $\left\{\delta f, \delta k_{1}, \delta k_{2}\right\}$ is consistent (at the linearized level). Equations of motion for the fluctuations and their asymptotic expansions in the UV $(\rho \rightarrow 0)$ and the IR $\left(y=\frac{1}{\rho}\right)$ are collected in appendix C. The expansions are characterized by $6 \mathrm{UV} / \mathrm{IR}$ parameters

$$
\begin{aligned}
\mathrm{UV}: & \left\{\delta f_{3,0}, \delta k_{2,3,0}, \delta k_{2,7,0}\right\} ; \\
\mathrm{IR}: & \left\{\delta f_{0}^{h}, \delta k_{1,0}^{h}, \delta k_{2,0}^{h}\right\},
\end{aligned}
$$

Without the loss of generality, we fix the overall normalization of the linearized $\chi$ SB fluctuations setting

$$
\delta f_{0}^{h} \equiv 1 .
$$

Note that we have only 5 parameters in (6.38), specifying a solution to 3 second-order differential equations (C.2)-(C.4) for $\left\{\delta f, \delta k_{1}, \delta k_{2}\right\}$ on $\mathcal{T}_{\text {decon }}^{s}$ background parameterized by $K_{0}$, with given $\mu / \Lambda$. For a generic value of $K_{0}$ (equivalently $T / \Lambda$ ) there is no solution: given $\mu / \Lambda, K_{0}$ must be tuned to identify the $\chi \mathrm{SB}$ normalizable mode. This tuned value of $K_{0}$, for the corresponding background Klebanov-Tseytlin black hole, would determine the temperature $T$ (A.59), which is precisely $T_{\chi \mathrm{SB}}$ at a given value $\mu / \Lambda$ :

$$
\frac{T_{\chi \mathrm{SB}}}{\Lambda}=\frac{f_{1}^{h}}{4 \pi \sqrt{h_{0}^{h}}} \times 2^{-1 / 4} e^{K_{0} / 2} .
$$



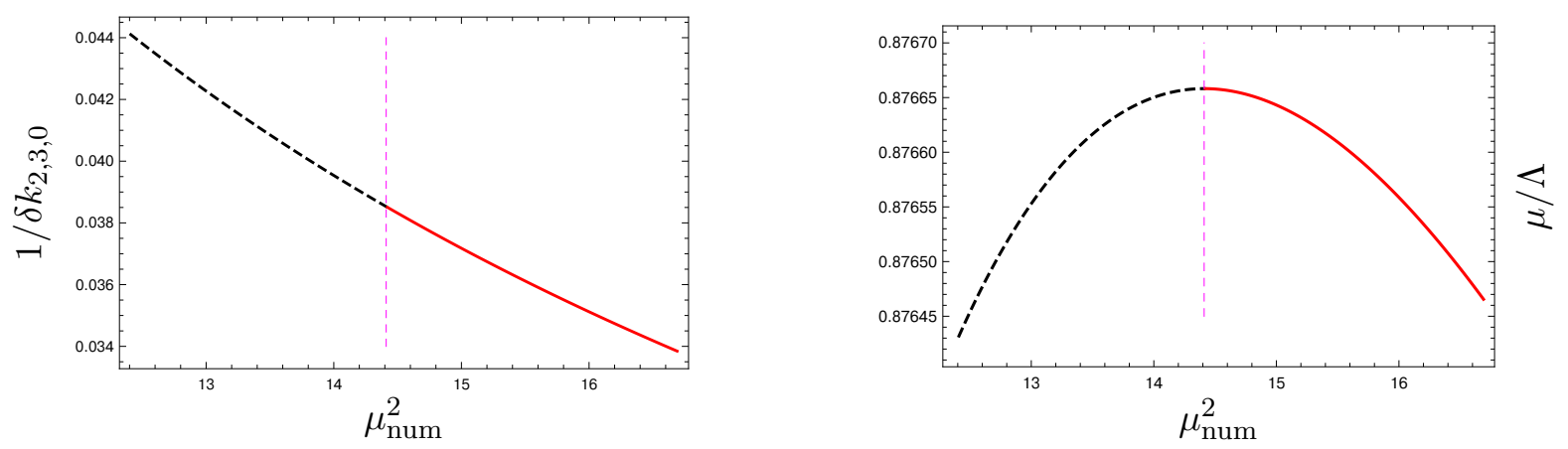

Figure 32. Variation of parameters (6.43) of the critical Klebanov-Tseytlin black hole with $\mu_{\text {num }}$ :

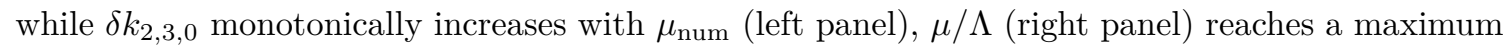
at $\mu_{\text {num }}=\mu_{\text {num, } \max }(6.44)$, represented by the vertical dashed magenta line.

The access to the numerical data for the chiral symmetry breaking for the black branes on the conifold [11] greatly simplifies our task: using the map (6.27), with (6.30) and (6.31), we identify from the data in [11] the onset of the chiral symmetry breaking at $\mu=0$ to occur at

$$
K_{0}=-0.43170(7), \quad f_{1}^{h}=3.8074(5), \quad h_{0}^{h}=0.14353(3),
$$

leading to (from (6.40))

$$
\left.\frac{T_{\chi \mathrm{SB}}}{\Lambda}\right|_{\mu=0}=0.54193(5)
$$

We continue to keep (6.30) and (6.31), and be vary $\mu$, simultaneously solving for the Klebanov-Tseytlin black hole $\left\{f, f_{c}, f_{3}, h, K, g\right\}$ and the $\chi \mathrm{SB}$ fluctuations $\left\{\delta f, \delta k_{1}, \delta k_{2}\right\}$. Note that in total we have 8 second-order $\mathrm{ODEs}^{25}$ (A.36), (A.37), (A.40), (A.41), (A.44), (C.2)-(C.4), and a single first order equation (A.45). To specify the solution we need to adjust $8 \times 2+1=17$ parameters. These are (see (A.55), (A.58) and (C.9)):

$$
\begin{aligned}
& \mathrm{UV}:\left\{K_{0}, f_{a, 1,0}, f_{c, 4,0}, g_{4,0}, f_{a, 6,0}, f_{c, 8,0}\right\} \quad \text { and } \quad\left\{\delta f_{3,0}, \delta k_{2,3,0}, \delta k_{2,7,0}\right\} ; \\
& \text { IR : }\left\{f_{a, 0}^{h}, f_{c, 0}^{h}, h_{0}^{h}, K_{1,0}^{h}, g_{0}^{h}, f_{1}^{h}\right\} \quad \text { and } \quad\left\{\delta k_{1,0}^{h}, \delta k_{2,0}^{h}\right\} \text {. }
\end{aligned}
$$

Given (6.43) we compute from (6.40) $T_{\chi \mathrm{SB}}(\mu) / \Lambda$ as a function of $\mu / \Lambda$.

We now explain the origin of $\mu_{K S}(6.17)$ - the terminal point of $T_{\chi \mathrm{SB}}(\mu)$ or $\hat{\mathcal{E}}_{\chi \mathrm{SB}}(\mu)$. Parameters (6.43), characterizing the critical Klebanov-Tseytlin black holes along with $\chi \mathrm{SB}$ fluctuations, are functions of $\mu_{\text {num }}$ - we use the subscript num to highlight that these are "numerical" values of $\mu$ in the computation scheme with (6.30) and (6.31). However, these parameters are not all monotonic functions of $\mu_{\text {num }}$. In figure 32 we present the variation of some parameters in (6.43) of the critical Klebanov-Tseytlin black hole with $\mu_{\text {num }}$ : while $\delta k_{2,3,0}$ monotonically increases with $\mu_{\text {num }}, \mu / \Lambda$ reaches a maximum at

$$
\mu_{\text {num }}^{2}=\mu_{\text {num }, \max }^{2} \equiv 14.41(0),
$$

\footnotetext{
${ }^{25}$ Recall that for the Klebanov-Tseytlin black hole $f_{a}=f_{b}=f_{3}, K_{1}=K_{2}=K$ and $K_{2}=1$.
} 

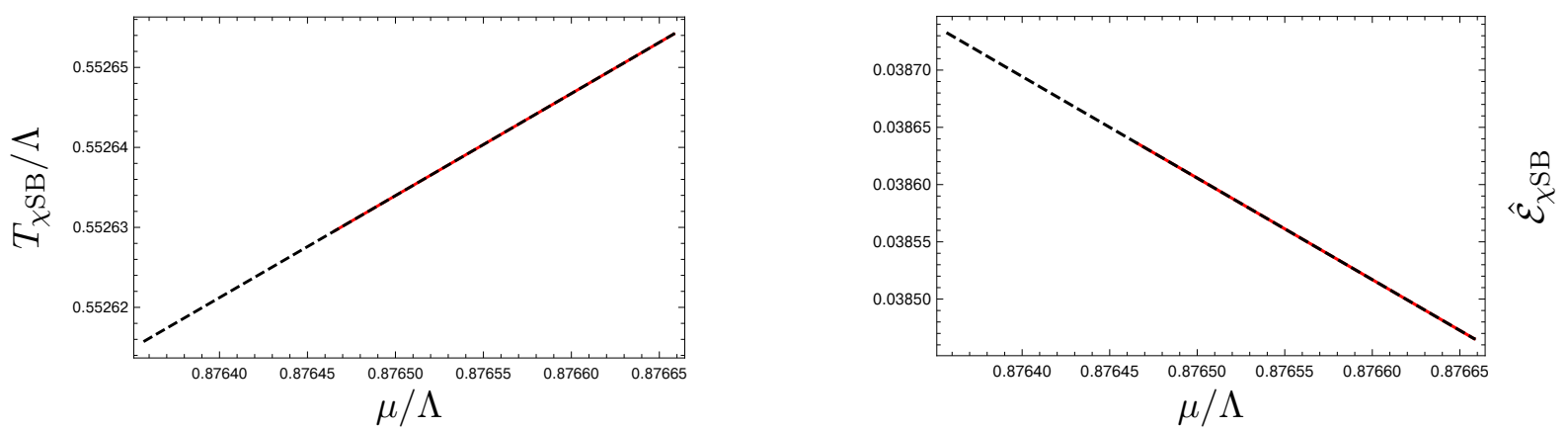

Figure 33. The critical Klebanov-Tseytlin black holes for $\mu_{\text {num }}<\mu_{\text {num,max }}$ (dashed black curves in figure 32) and $\mu_{\text {num }}>\mu_{\text {num,max }}$ (red curves in figure 32) have identical values of $T_{\chi \mathrm{SB}} / \Lambda$ (left panel) and $\hat{\mathcal{E}}_{\chi \mathrm{SB}}$ (right panel) as a function of $\mu / \Lambda$.

represented by the vertical dashed magenta line. This maximal value of $\mu / \Lambda$ is precisely $\mu_{K S} / \Lambda$ - there is no instability to $\chi \mathrm{SB}$ fluctuations of Klebanov-Tseytlin black holes for $\mu / \Lambda>\mu_{K S} / \Lambda$. A remarkable test on our numerics, the critical Klebanov-Tseytlin black holes for $\mu_{\text {num }}<\mu_{\text {num,max }}$ (black dashed curves in figure 32) and for $\mu_{\text {num }}>\mu_{\text {num,max }}$ (red curves in figure 32 ) are physically identical: in figure 33 we plot $T_{\chi \mathrm{SB}} / \Lambda$ (left panel) and $\hat{\mathcal{E}}_{\chi \mathrm{SB}}$ (right panel) of these two branches as a function of $\mu / \Lambda$ - there is a perfect overlap.

It is possible to set up numerics in such a way that we vary directly $\hat{\mu} \equiv \mu / \Lambda$ and thus set

$$
\mu \equiv 2^{1 / 4} e^{-K_{0} / 2} \hat{\mu}
$$

In this numerical scheme we have been unable to extend the critical line of the KlebanovTseytlin black holes past $\hat{\mu}=\mu_{K S} / \Lambda$ as well.

\subsubsection{Klebanov-Strassler black holes at $\mu \neq 0$}

In section 6.3.2 we constructed the set of critical Klebanov-Tseytlin black holes, labeled by $\mu / \Lambda \in\left[0, \mu_{K S} / \Lambda\right]$, unstable to spontaneous chiral symmetry breaking. Each point of $T_{\chi \mathrm{SB}}(\mu)$ (or $\hat{\mathcal{E}}_{\chi \mathrm{SB}}(\mu)$ ) of the phase diagrams discussed in sections 6.1 and 6.2 is a bifurcation point for the branch of Klebanov-Strassler black holes, equivalently $\mathcal{T}_{\text {decon }}^{b}$ the thermal deconfined states of the cascading gauge theory with the spontaneously broken chiral symmetry.

Given a bifurcation point on the critical Klebanov-Tseytlin black hole branch, the construction of the Klebanov-Strassler black hole branch is straightforward. In fact the procedure is identical to the one employed for the black branes in [4], or to the construction of $\mathcal{V}_{A}^{b}$ vacua in section 5.3. An important subtlety is that we need to keep $\mu / \Lambda$ fixed, allowing for the variation of the temperature/energy density of the Klebanov-Strassler black hole. This is done as in section 6.3.1: we vary both $K_{0}$ and $\mu$ along the line (6.34), where the KS black hole branch label $\hat{\mu}$ is picked from the range $\hat{\mu} \in\left[0, \mu_{K S} / \Lambda\right]$. Specific procedural steps are as follows. 
From (6.43) we identify the susceptibilities (compare with (5.35)) as

$$
\left\{\chi_{f_{3,0}}, \chi_{k_{2,3,0}}, \chi_{k_{2,7}, 0}, \chi_{k_{1,0}^{h}}, \chi_{k_{2,0}^{h}}\right\} \equiv\left\{\frac{\delta f_{3,0}}{\delta f_{0}^{h}}, \frac{\delta k_{2,3,0}}{\delta f_{0}^{h}}, \frac{\delta k_{2,7,0}}{\delta f_{0}^{h}}, \frac{\delta k_{1,0}^{h}}{\delta f_{0}^{h}}, \frac{\delta k_{2,0}^{h}}{\delta f_{0}^{h}}\right\},
$$

remembering (6.39).

Similar to (5.38), we denote the amplitude of the chiral symmetry breaking condensate (see $(6.37))$

$$
\delta f_{0}^{h} \equiv \frac{1}{2}\left(f_{a, 0}^{h}-f_{b, 0}^{h}\right)=\lambda
$$

Then,

$$
\left\{\delta f_{3,0} \delta k_{2,3,0}, \delta k_{2,7,0}, \delta k_{1,0}^{h}, \delta k_{2,0}^{h}\right\}=\lambda\left\{\chi_{f_{3,0}}, \chi_{k_{2,3,0}}, \chi_{k_{2,7,0}}, \chi_{k_{1,0}^{h}}, \chi_{k_{2,0}^{h}}\right\}+\mathcal{O}\left(\lambda^{2}\right) .
$$

Using (6.37), keeping (6.30) fixed, and correlating $\mu$ and $K_{0}$ variation as in (6.34), we find to $\mathcal{O}\left(\lambda^{2}\right)$ :

$$
\begin{aligned}
K_{0} & =K_{0}^{\text {crit }}+\mathcal{O}\left(\lambda^{2}\right), \quad f_{a, 1,0}=f_{a, 1,0}^{\text {crit }}+\mathcal{O}\left(\lambda^{2}\right), \quad f_{a, 3,0}=\chi_{f_{3,0}} \lambda+\mathcal{O}\left(\lambda^{2}\right), \\
k_{2,3,0} & =\lambda \chi_{k_{2,3,0}}+\mathcal{O}\left(\lambda^{2}\right), \quad f_{c, 4,0}=f_{c, 4,0}^{\text {crit }}+\mathcal{O}\left(\lambda^{2}\right), \\
g_{4,0} & =g_{4,0}^{\text {crit }}+\mathcal{O}\left(\lambda^{2}\right), \quad f_{a, 6,0}=f_{a, 6,0}^{\text {crit }}+\mathcal{O}\left(\lambda^{2}\right), \quad k_{2,7,0}=\chi_{k_{2,7,0}} \lambda+\mathcal{O}\left(\lambda^{2}\right), \\
f_{c, 8,0} & =f_{c, 8,0}^{\text {crit }}+\mathcal{O}\left(\lambda^{2}\right), \quad f_{a, 0}^{h}=f_{a, 0}^{h, \text { crit }}+\lambda+\mathcal{O}\left(\lambda^{2}\right), \\
f_{b, 0}^{h} & =f_{a, 0}^{h, \text { crit }}-\lambda+\mathcal{O}\left(\lambda^{2}\right), \quad f_{c, 0}^{h}=f_{c, 0}^{h, \text { crit }}+\mathcal{O}\left(\lambda^{2}\right), \quad h_{0}^{h}=h_{0}^{h, \text { crit }}+\mathcal{O}\left(\lambda^{2}\right), \\
K_{1,0}^{h} & =K_{1,0}^{h, \text { crit }}+\chi_{k_{1,0}^{h}} \lambda+\mathcal{O}\left(\lambda^{2}\right), \quad K_{2,0}^{h}=1+\chi_{k_{2,0}^{h}} \lambda+\mathcal{O}\left(\lambda^{2}\right), \\
K_{3,0}^{h} & =K_{1,0}^{h, \text { crit }}-\chi_{k_{1,0}^{h}}^{h} \lambda+\mathcal{O}\left(\lambda^{2}\right), \quad g_{0}^{h}=g_{0}^{h, \text { crit }}+\mathcal{O}\left(\lambda^{2}\right), \quad f_{1}^{h}=f_{1}^{h, \text { crit }}+\mathcal{O}\left(\lambda^{2}\right),
\end{aligned}
$$

where the superscript ${ }^{\text {crit }}$ stands for the values of the corresponding parameters in (6.43) along the critical line of the Klebanov-Tseytlin black holes.

We construct fully nonlinear in $\lambda \mathcal{T}_{\text {decon }}^{b}$ states, i.e., the Klebanov-Strassler black holes, using the linearized approximation (6.49) as a seed.

An important check on our numerics is the validity of the first law of thermodynamics (A.91). For example, the right panel of figure 35 verifies this for the Klebanov-Strassler black hole at $\mu=\mu_{1}(6.18)$ - the accuracy is $\sim 10^{-5}$ and better. This is a typical accuracy achieved for the Klebanov-Strassler black holes at $\mu / \Lambda \neq 0$.

\subsubsection{Computation of $T_{c}(\mu)$}

$T_{c}(\mu)$ is the temperature of the confinement deconfinement phase transition. There are distinct confined states: $\mathcal{T}_{\text {con,B }}$ and $\mathcal{T}_{\text {con,A }}^{s}$ (see figure 22) and $\mathcal{T}_{\text {con,A }}^{b}$ (see figure 23). Correspondingly, there are distinct curves $T_{c}(\mu)$ for the transition between the deconfined chirally symmetric states $\mathcal{T}_{\text {decon }}^{s}$, i.e., the Klebanov-Tseytlin black holes, and these confined states. Computation of the deconfined temperature is a numerically consuming procedure, albeit very straightforward: we follow section 6.3.1 to generate spectra of Klebanov-Tseytlin black holes labeled by $\mu / \Lambda$.

At fixed $\hat{\mu} \equiv \mu / \Lambda$, we compute the reduced free energy density $\hat{\mathcal{F}}_{\mathcal{T}_{\text {decon }}^{s}}(T ; \hat{\mu})($ see $(6.1))$ of $\mathcal{T}_{\text {decon }}^{s}$ states, using the expressions in appendices A.4.4 and A.4.5. 
The reduced free energy density of the confined states is temperature independent, and is simply the reduced vacuum energy density of $\mathcal{V}_{B}, \mathcal{V}_{A}^{s}$ or $\mathcal{V}_{A}^{b}$ (A.82) at the corresponding value of $\hat{\mu}$.

For example, for $\mathcal{T}_{\text {decon }}^{s} \leftrightarrow \mathcal{T}_{\text {con,B }}$ transition reported in figure $22, T_{c}(\mu)$ is determined from

$$
\left.\hat{\mathcal{F}}_{\mathcal{T}_{\text {decon }}^{s}}(T ; \hat{\mu})\right|_{T=T_{c}(\mu)}=\mathcal{V}_{B}(\hat{\mu})
$$

We replicated (6.50) for the transitions $\mathcal{T}_{\text {decon }}^{s} \leftrightarrow \mathcal{T}_{\text {con,A }}^{s}$ and $\mathcal{T}_{\text {decon }}^{s} \leftrightarrow \mathcal{T}_{\text {con,A }}^{b}$, and extract the corresponding confinement/deconfinement temperatures $T_{c}$.

\subsection{Conifold black holes at $\{T, \mu\} \gg \Lambda$}

In this section we extend the work on black branes on the conifold with fluxes in the limit $T \gg \Lambda$ of $[25,38,39]$ to Klebanov-Tseytlin black holes, i.e., $\mathcal{T}_{\text {decon }}^{s}$ states. We work to the leading order in the limit $\{T, \mu\} \gg \Lambda$, without any particular hierarchy between $T$ and $\mu$. The main motivation for this analysis is to have yet another check on the numerics and the holographic renormalization of the model.

Chiral symmetry is unbroken in the limit $T \gg \Lambda$ [23], so our starting point are the conformal $\mathcal{T}_{\text {decon }}^{s}$ thermal states of section 4.2. As in [39], we use a perturbative in $P^{2} g_{s} / \hat{K}_{0}$ ansatz to solve (A.36)-(A.45):

$$
\begin{aligned}
f & =\frac{4\left(\hat{f}_{a, 1,0} \rho+1\right)\left(\hat{K}_{0} \mu^{2} \rho^{2}+2 \hat{f}_{a, 1,0}^{2} \rho^{2}+4 \hat{f}_{a, 1,0} \rho+4\right)}{\left(\hat{f}_{a, 1,0} \rho+2\right)^{4}} \times\left[1+\sum_{n=1}^{\infty}\left(\frac{P^{2} g_{s}}{\hat{K}_{0}}\right)^{2} f_{n}(\rho)\right] \\
f_{a} & =f_{b}=\frac{\left(\hat{f}_{a, 1,0} \rho+2\right)^{2}}{4} \times\left[1+\sum_{n=1}^{\infty}\left(\frac{P^{2} g_{s}}{\hat{K}_{0}}\right)^{2} f_{3, n}(\rho)\right] \\
f_{c} & =\frac{\left(\hat{f}_{a, 1,0} \rho+2\right)^{2}}{4} \times\left[1+\sum_{n=1}^{\infty}\left(\frac{P^{2} g_{s}}{\hat{K}_{0}}\right)^{2} f_{c, n}(\rho)\right] \\
h & =\frac{4 \hat{K}_{0}}{\left(\hat{f}_{a, 1,0} \rho+2\right)^{4}} \times\left[1+\sum_{n=1}^{\infty}\left(\frac{P^{2} g_{s}}{\hat{K}_{0}}\right)^{2} h_{n}(\rho)\right], \\
K_{1} & =K_{3}=\hat{K}_{0} \times\left[1+\sum_{n=1}^{\infty}\left(\frac{P^{2} g_{s}}{\hat{K}_{0}}\right)^{2} k_{1, n}(\rho)\right], \quad K_{2} \equiv 1 \\
g & =g_{s} \times\left[1+\sum_{n=1}^{\infty}\left(\frac{P^{2} g_{s}}{\hat{K}_{0}}\right)^{2} g_{n}(\rho)\right],
\end{aligned}
$$

were we used the conformal solution (4.22) in the limit $\Lambda / T \rightarrow 0$. It is convenient to introduce

$$
x \equiv \frac{\hat{f}_{a, 1,0}}{2} \rho, \quad q \equiv \frac{\mu^{2} \hat{K}_{0}}{\hat{f}_{a, 1,0}^{2}}, \quad z \equiv \frac{P^{2} g_{s}}{\hat{K}_{0}} .
$$

All the functions $f_{n}, f_{3, n}, f_{c, n}, h_{n}, k_{1, n}, g_{n}$ become the functions of the new radial coordinate $x$, with the parametric dependence on $q$. We present the explicit equations and the asymptotic expansions for these functions for $n=1$ in appendix $\mathrm{D}$. 
Using (6.52), the asymptotic expansions (D.11)-(D.16), we identify ${ }^{26}$ (compare (A.55) and (A.58))

$$
\begin{aligned}
K_{0}= & \hat{K}_{0}\left(1-2 \ln \frac{\hat{f}_{a, 1,0}}{2} z\right), \quad f_{a, 1,0}=\hat{f}_{a, 1,0}\left(1+\frac{1}{2} \hat{f}_{c, 1.0} z+\mathcal{O}\left(z^{2}\right)\right), \\
f_{4,0}= & \frac{1}{16} \hat{f}_{a, 1,0}^{4}(2 q-1)+\frac{1}{96} \hat{f}_{a, 1,0}^{4}\left(q(8 q \ln 2+36 \ln 2+3 q)-4 q(2 q+9) \ln \hat{f}_{a, 1,0}\right. \\
& \left.+6 \hat{f}_{1,4,0}\right) z+\mathcal{O}\left(z^{2}\right), \quad f_{c, 4,0}=-\frac{1}{144} \hat{f}_{a, 1,0}^{4}\left(5 q^{2}+3 q-3\right) z+\mathcal{O}\left(z^{2}\right), \\
f_{a, 0}^{h}= & \frac{1}{4} \hat{f}_{a, 1,0}^{2}\left(1+f_{3,1,0}^{h} z+\mathcal{O}(z)^{2}\right), \quad f_{c, 0}^{h}=\frac{1}{4} \hat{f}_{a, 1,0}^{2}\left(1+f_{c, 1,0}^{h} z+\mathcal{O}(z)^{2}\right), \\
h_{0}^{h}= & \frac{4 \hat{K}_{0}}{\hat{f}_{a, 1,0}^{4}}\left(1+\hat{h}_{1,0}^{h} z+\mathcal{O}\left(z^{2}\right)\right), \quad f_{1}^{h}=\frac{4(q+2)}{\hat{f}_{a, 1,0}}\left(1+\hat{f}_{1,0}^{h} z+\mathcal{O}\left(z^{2}\right)\right),
\end{aligned}
$$

where we identified only the parameters necessary to compute the energy density $\mathcal{E}$ (A.86), the entropy density $s$ (A.89) and the temperature $T$ (A.59).

Using (2.48), (6.53) and (6.52) we identify

$$
\frac{\mu^{2}}{\Lambda^{2}}=2^{3 / 2} P^{2} g_{s} \frac{q}{\hat{K}_{0}} e^{\frac{\hat{K}_{0}}{P^{2} g_{s}}}
$$

We produce the numerical data for Klebanov-Tseytlin black holes using $q$ as a label (see appendix D) - to discuss thermodynamics of a fixed theory we must keep $\left\{P, g_{s}, \mu, \Lambda\right\}$ constant as the temperature $T$ varies. ${ }^{27}$ This obviously necessitates that

$$
\hat{K}_{0}=\hat{K}_{0}(q) \quad \Longrightarrow \quad \frac{d \hat{K}_{0}}{d q}=-\frac{P^{2} g_{s}}{q} \times \frac{\hat{K}_{0}}{\hat{K}_{0}-P^{2} g_{s}} .
$$

The fact that $\hat{K}_{0}$ must depend on temperature, compactification scale is not new and was first observed in [23].

Notice that at $q=0$, i.e., the limit of the Klebanov-Tseytlin black brane,

$$
\left.f_{4,0}\right|_{q=0}=-\frac{1}{16} \hat{f}_{a, 1,0}^{4}\left(1-\hat{f}_{1,4,0} z+\mathcal{O}\left(z^{2}\right)\right) .
$$

As discussed earlier, see (6.30), $f_{4,0}$ sets the overall mass scale, and can be adjusted at will, without affecting physics, provided we express observables as dimensionless quantities. In (6.30) $f_{4,0}$ was fixed matching to (fully nonlinear) numerics used for black branes, i.e., at $q=0$ and finite $T / \Lambda$. Here, we do the same, matching $f_{4,0}$ to the perturbative black brane numerics of [3]. We omit the technical details and simply present the answer:

$$
\hat{f}_{a, 1,0}=2, \quad \hat{f}_{1,4,0}=\frac{1}{3} .
$$

\footnotetext{
${ }^{26}$ Note that the relation for $K_{0}$ is exact to all orders in $z$, provided the additive integration constants are set to zero for all $k_{1, n}$ functions, see (D.10) for $k_{1,1}$.

${ }^{27}$ Here, the temperature variation is induced by variation of $q$.
} 
Using (6.52), (6.60), (6.53)-(6.56), and the thermodynamic expressions of appendices A.4.4 and A.4.5 we find

$$
\begin{aligned}
\frac{8 \pi G_{5} \mathcal{E}}{\mu^{4}} & =\frac{\hat{K}_{0}^{2}}{32}\left(\frac{q^{2}+3 q+3}{q^{2}}+\frac{54 \hat{f}_{c, 1,0}-2 q-69}{6 q} \frac{P^{2} g_{s}}{\hat{K}_{0}}+\mathcal{O}\left(z^{2}\right)\right), \\
\frac{8 \pi G_{5} s}{\mu^{3}} & =\frac{\pi \hat{K}_{0}^{2}}{8 q^{3 / 2}}\left(1+\frac{\hat{h}_{1,0}^{h}+4 \hat{f}_{3,1,0}^{h}+\hat{f}_{c, 1,0}^{h}}{2} \frac{P^{2} g_{s}}{\hat{K}_{0}}+\mathcal{O}\left(z^{2}\right)\right), \\
\frac{T}{\mu} & =\frac{q+2}{2 \pi q^{1 / 2}}\left(1+\frac{2 \hat{f}_{1,0}^{h}-\hat{h}_{1,0}^{h}}{2} \frac{P^{2} g_{s}}{\hat{K}_{0}}+\mathcal{O}\left(z^{2}\right)\right) .
\end{aligned}
$$

The first law of thermodynamics in the form $d \mathcal{E}=T d s$, keeping $\left\{P, g_{s}, \mu, \Lambda\right\}$ constant, and using (6.58), is automatically satisfied at $\mathcal{O}\left(z^{0}\right)$, while at $\mathcal{O}(z)$ it leads to the constraint:

$$
0=\mathcal{F} \mathcal{L}_{[1], 2}(q) q^{2}+\mathcal{F} \mathcal{L}_{[1], 1}(q) q+\mathcal{F} \mathcal{L}_{[1], 0}(q) \equiv \mathcal{F} \mathcal{L}_{[1]}(q)
$$

with

$$
\begin{aligned}
& \mathcal{F} \mathcal{L}_{[1], 2}=-\frac{4}{3}\left(\hat{f}_{3,1,0}^{h}\right)^{\prime}-\frac{1}{3}\left(\hat{f}_{c, 1,0}^{h}\right)^{\prime}+3\left(\hat{f}_{c, 1,0}\right)^{\prime}-\frac{1}{3}\left(\hat{h}_{1,0}^{h}\right)^{\prime}-\frac{2}{3}, \\
& \mathcal{F} \mathcal{L}_{[1], 1}=2 \hat{f}_{3,1,0}^{h}+\frac{1}{2} \hat{f}_{c, 1,0}^{h}-\frac{8}{3}\left(\hat{f}_{3,1,0}^{h}\right)^{\prime}-\frac{2}{3}\left(\hat{f}_{c, 1,0}^{h}\right)^{\prime}-\frac{2}{3}\left(\hat{h}_{1,0}^{h}\right)^{\prime}+\hat{f}_{1,0}^{h}-3 \hat{f}_{c, 1,0}+\frac{19}{6}, \\
& \mathcal{F} \mathcal{L}_{[1], 0}=4 \hat{f}_{3,1,0}^{h}+\hat{f}_{c, 1,0}^{h}+2 \hat{f}_{1,0}^{h}+\frac{2}{3},
\end{aligned}
$$

where $^{\prime} \equiv d / d q$. In figure 37 we check the first law of thermodynamics (6.64) presenting

$$
\mathcal{C}_{[1]} \equiv \frac{\mathcal{F} \mathcal{L}_{[1]}(q)}{\max \left[\mathcal{F} \mathcal{L}_{[1], 2}(q), \mathcal{F} \mathcal{L}_{[1], 1}(q), \mathcal{F} \mathcal{L}_{[1], 0}(q)\right]},
$$

as a function of $q$. We find that $\mathcal{C}_{[1]} \sim 10^{-8}$ for the range $q \in[0,0.8] \cup[1.2,2]$. We deliberately excluded the region around $q=1$ for an important reason: it turns out that almost all parameters in (D.17) diverge as $q \rightarrow 1$ (both from above and below). As we demonstrate now, this fact has interesting implications for the leading corrections to the confinement/deconfinement temperature $T_{c}$ for the transition $\mathcal{T}_{\text {decon }}^{s} \leftrightarrow \mathcal{T}_{\text {con }}^{s}$ in the limit $\{T, \mu\} \gg \Lambda$.

We will not presents results for the divergence of all the parameters in (D.17), and focus instead on two combinations $\mathcal{C}_{1}$ and $\mathcal{C}_{2}$, which will be relevant for the computation of the leading in $P^{2} g_{s} / \hat{K}_{0}$ corrections to the Hawking-Page temperature (4.31):

$$
\begin{aligned}
& \mathcal{C}_{1}(q) \equiv\left(2 \hat{f}_{1,0}^{h}-9 \hat{f}_{c, 1,0}+\hat{f}_{c, 1,0}^{h}+4 \hat{f}_{3,1,0}^{h}\right) q+4 \hat{f}_{1,0}^{h}+2 \hat{f}_{c, 1,0}^{h}+8 \hat{f}_{3,1,0}^{h}, \\
& \mathcal{C}_{2}(q) \equiv 2 \hat{f}_{1,0}^{h}-\hat{h}_{1,0}^{h} .
\end{aligned}
$$

In figure 34 the blue curves represent $\mathcal{C}_{1}^{-1}$ (the left panel) and $\mathcal{C}_{2}^{-1}$ (the right panel) as a function of $q$. The dashed black lines represent the slopes to the curves at $q=1$ :

$$
\begin{array}{ll}
\mathcal{C}_{1}^{-1}=\delta_{1}(q-1)+\mathcal{O}\left((q-1)^{2}\right), & \delta_{1}=0.26695(6), \\
\mathcal{C}_{1}^{-2}=\delta_{2}(q-1)+\mathcal{O}\left((q-1)^{2}\right), & \delta_{2}=0.80086(7)
\end{array}
$$



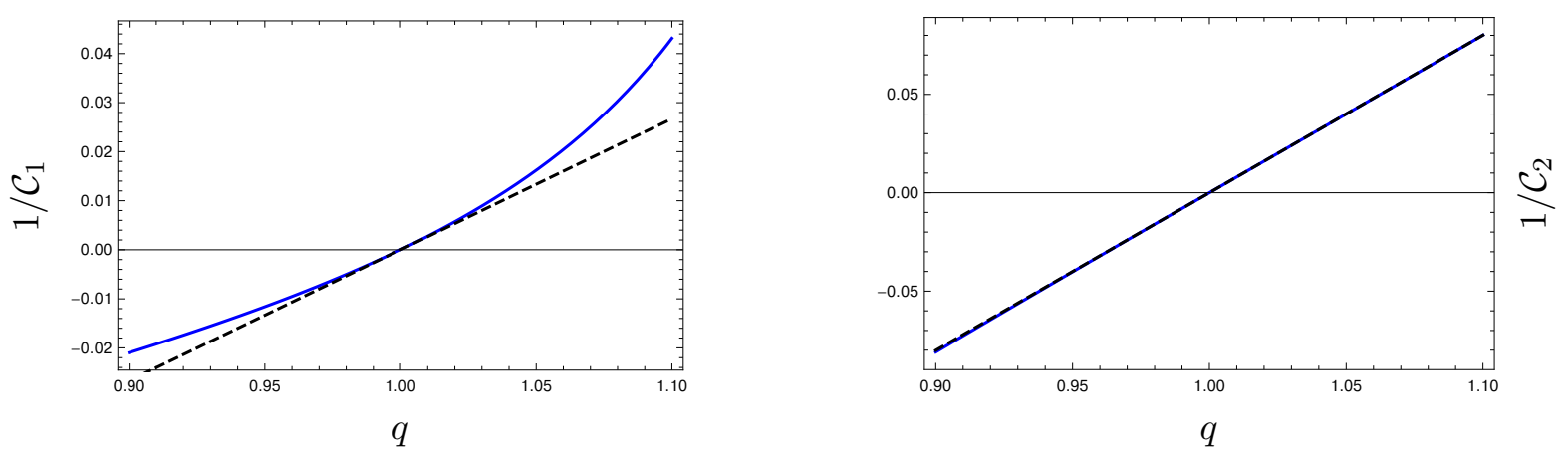

Figure 34. Certain linear combinations of the parameters in (D.17), $\mathcal{C}_{1}$ (the left panel, (6.67)) and $\mathcal{C}_{2}$ (the right panel, (6.68)), diverge as $q \rightarrow 1$. The dashed black lines are the tangents to the curves $1 / \mathcal{C}_{1}$ and $1 / \mathcal{C}_{2}$ at $q=1$

Note a serendipitous numerical fact:

$$
3 \delta_{1}-\delta_{2}=-1.0(5) \times 10^{-9},
$$

i.e., with very good numerical accuracy $3 \delta_{1}=\delta_{2}$. We are now ready to compute the correction to $T_{c}$ as $\mu / \Lambda \rightarrow \infty$ for Klebanov-Tseytlin black holes. From (6.61)-(6.63) we have

$$
\begin{aligned}
\frac{8 \pi G_{5} \mathcal{F}\left[\mathcal{T}_{\text {decon }}^{s}\right]}{\mu^{4}} & =\frac{8 \pi G_{5} \mathcal{E}}{\mu^{4}}-\frac{8 \pi G_{5} s}{\mu^{3}} \times \frac{T}{\mu} \\
& =\frac{\hat{K}_{0}^{2}}{32}\left(\frac{q^{2}+q-1}{q^{2}}-\frac{\left(6 \mathcal{C}_{1}+2 q^{2}+69 q\right)}{6 q^{2}} \frac{P^{2} g_{s}}{\hat{K}_{0}}+\mathcal{O}\left(z^{2}\right)\right),
\end{aligned}
$$

where $\mathcal{C}_{1}$ is given (6.67). For the confined state,

$$
\frac{8 \pi G_{5} \mathcal{F}\left[\mathcal{T}_{\text {con }}^{s}\right]}{\mu^{4}}=\frac{8 \pi G_{5} \mathcal{E}\left[\mathcal{V}_{A}^{s}\right]}{\mu^{4}}=\frac{\hat{K}_{0}^{2}}{32}\left(1+\gamma \frac{P^{2} g_{s}}{\hat{K}_{0}}+\mathcal{O}\left(z^{2}\right)\right),
$$

where the coefficient $\gamma$ was computed $\operatorname{in}^{28}[10]$

$$
\gamma=-2.2725(9)
$$

Confinement/deconfinement transition occurs when

$$
\mathcal{F}\left[\mathcal{T}_{\text {con }}^{s}\right]=\mathcal{F}\left[\mathcal{T}_{\text {decon }}^{s}\right] .
$$

To leading order in $P^{2} g_{s} / \hat{K}_{0}$ it happens at

$$
\left.q\right|_{\text {leading order }}=1
$$

Precisely because $\mathcal{C}_{1}$ diverges as $\propto 1 /(q-1)$ as $q \rightarrow 1$, the leading correction to (6.75) in the limit $\mu / \Lambda \rightarrow \infty$ are nonanalytic in $P^{2} g_{s} / \hat{K}_{0}$. Using (6.69), we find from (6.74)

$$
\left.q\right|_{\text {con/decon }}=1 \pm \delta_{1}^{-1 / 2}\left(\frac{P^{2} g_{s}}{\hat{K}_{0}}\right)^{1 / 2}+\mathcal{O}\left(\frac{P^{2} g_{s}}{\hat{K}_{0}}\right) .
$$

\footnotetext{
${ }^{28}$ We independently verified that result here.
} 
To compute the correction to the transition temperature (6.63) due to (6.76) we need to remember that $\mathcal{C}_{2}(q)$ (6.68) diverges as $q \rightarrow 1$ as well - see (6.69). We find

$$
\begin{aligned}
\frac{T_{c}}{\mu} & =\frac{3}{2 \pi} \pm \frac{3 \delta_{1}-\delta_{2}}{4 \delta_{1}^{1 / 2} \delta_{2} \pi}\left(\frac{P^{2} g_{s}}{\hat{K}_{0}}\right)^{1 / 2}+\mathcal{O}\left(\frac{P^{2} g_{s}}{\hat{K}_{0}}\right) \\
& =\frac{3}{2 \pi}+0 \times\left(\frac{1}{2 \ln \frac{\mu}{\Lambda}}\right)^{1 / 2}+\mathcal{O}\left(\frac{1}{2 \ln \frac{\mu}{\Lambda}}\right),
\end{aligned}
$$

where in the second equality we used $3 \delta_{1}-\delta_{2}=0$ (motivated by (6.70)) and (6.57) at $q=1$ and to leading order in $\mu / \Lambda \rightarrow \infty$. Thus, to compute the leading in the limit $\mu / \Lambda \rightarrow \infty$ nonvanishing correction to the confinement/deconfinement transition temperature $T_{c}^{\text {conformal }}$, and compare with results reported in figure 24 we need to develop perturbative solution to the Klebanov-Tseytlin black hole (6.51) to order $n=2$. We will not pursue this computation here.

\section{Conclusion and open questions}

In this paper we studied the vacua and the black holes on the conifold of Type IIB supergravity with fluxes. These background geometries realize the holographic dual to vacua and thermal states of the cascading gauge theory [2] on a 3-sphere. We uncovered rich phase diagrams in the canonical and microcanonical ensembles, identified distinct confinement/deconfinement phase transitions, spontaneous breaking of the chiral symmetry in vacua and in thermal states. Further details are provided in sections 5 and 6 .

We list now open question for the future work.

- In a review section 4.1 , see figure 4 in particular, we recalled that $A d S_{5} \times T^{1,1}$ black holes we unstable to localization on $T^{1,1}[27-29]$. There are no studies in the literature on the localization instabilities in the presence of internal (3-form) fluxes on $T^{1,1}$.

- We still do not know what is the end point of the chiral symmetry breaking instability of Klebanov-Tseytlin black branes, i.e., when $\mu=0$. Although Klebanov-Strassler black branes are the preferred states in the microcanonical ensemble [4], their translational invariant horizon is unstable to gravitational perturbations, the sound modes in the dual cascading gauge theory plasma.

- In holography, there is a simple relation between the thermodynamic and the dynamical instabilities of the black branes [41]. In this work we established that KlebanovStrassler black holes, as well as some branches of the Klebanov-Tseytlin black holes, have a negative specific heat, i.e., are thermodynamically unstable. Whether or not they are dynamically unstable (when $\mu \neq 0$ ) is an open question.

- In this paper we used holography to study the vacua and the thermal states of the interesting strongly coupled quantum field theory which has a confinement and a spontaneous breaking of a symmetry. It would be very interesting to understand 
how to enlarge the holographic duality used here, and include the background magnetic field. ${ }^{29}$ This would open the possibility to explore confinement/deconfinement and chiral symmetry breaking in the presence of the magnetic field in a top-down holographic model. A challenge is to identify a simple enough consistent truncation including a bulk gauge field for the class of models in [47, 48].

- In this paper we identified certain conifold vacua which are unstable to the spontaneous breaking of the chiral symmetry, and could 'thermalize' into Klebanov-Strassler black holes. It would be interesting to simulate this dynamics.

- There is an interesting interval in the $S^{3}$ compactification scale of the cascading gauge theory, namely $\mu \in\left[\mu_{K S}, \mu_{u}\right]$. In this range, chirally symmetric vacua of the conifold with fluxes are unstable to symmetry breaking fluctuations, yet, there are no Klebanov-Strassler black holes, see figure 28. What is the fate of these vacua instabilities?

\section{Acknowledgments}

This research is supported in part by Perimeter Institute for Theoretical Physics. Research at Perimeter Institute is supported in part by the Government of Canada through the Department of Innovation, Science and Economic Development Canada and by the Province of Ontario through the Ministry of Colleges and Universities. This work was further supported by NSERC through the Discovery Grants program.

\section{A Equations of motion, asymptotic expansions, and the holographic renormalization}

\section{A.1 Case (A): horizonless warped deformed conifold with fluxes}

Using the metric ansatz (3.19) we derive from (3.1) the following equations of motion:

$$
\begin{aligned}
0= & f_{1}^{\prime \prime}+\frac{2\left(f_{1}^{\prime}\right)^{2}}{f_{1}}-\frac{f_{1}^{\prime}}{2}\left(\frac{6}{\rho}-\frac{2 f_{b}^{\prime}}{f_{b}}-\frac{2 f_{a}^{\prime}}{f_{a}}-\frac{f_{c}^{\prime}}{f_{c}}\right)-\frac{2 h \mu^{2}}{f_{1}} \\
0= & f_{a}^{\prime \prime}-\frac{3 f_{a}}{2 f_{1}^{2}}\left(f_{1}^{\prime}\right)^{2}-\frac{1}{8 f_{a}}\left(f_{a}^{\prime}\right)^{2}-\frac{f_{a}}{8 f_{b}^{2}}\left(f_{b}^{\prime}\right)^{2}+\frac{f_{a}}{8 h^{2}}\left(h^{\prime}\right)^{2}+\frac{f_{a}}{8 g^{2}}\left(g^{\prime}\right)^{2}+\frac{g P^{2}}{36 h f_{b}}\left(K_{2}^{\prime}\right)^{2} \\
& +\frac{5}{32 g h f_{a} P^{2}}\left(K_{3}^{\prime}\right)^{2}-\frac{3 f_{a}}{32 g h f_{b}^{2} P^{2}}\left(K_{1}^{\prime}\right)^{2}+\left(\frac{3 f_{a}^{\prime}}{2 f_{1}}-\frac{3 h^{\prime} f_{a}}{4 h f_{1}}-\frac{3 f_{c}^{\prime} f_{a}}{4 f_{1} f_{c}}-\frac{3 f_{b}^{\prime} f_{a}}{2 f_{b} f_{1}}-\frac{3 f_{a}}{2 r f_{1}}\right) f_{1}^{\prime} \\
& +\left(\frac{f_{a}^{\prime}}{4 f_{c}}-\frac{f_{b}^{\prime} f_{a}}{4 f_{b} f_{c}}\right) f_{c}^{\prime}+\frac{f_{a}^{\prime} f_{b}^{\prime}}{2 f_{b}}+\frac{h^{\prime} f_{a}}{h \rho}-\frac{3 f_{a}^{\prime}}{\rho}-\frac{9\left(K_{1}-K_{3}\right)^{2}}{64 f_{b} h g f_{c} \rho^{2} P^{2}}-\frac{K_{1}^{2}}{8 f_{b}^{2} h^{2} f_{a} f_{c} \rho^{2}}+\frac{5 f_{a}}{\rho^{2}} \\
& -\frac{g\left(K_{2}^{2}\left(5 f_{a}^{2}-3 f_{b}^{2}\right)+12 f_{b}^{2}\left(K_{2}-1\right)\right) P^{2}}{8 h f_{b}^{2} f_{a} f_{c} \rho^{2}}-\frac{K_{2}\left(K_{1}-K_{3}\right)\left(K_{2}\left(K_{1}-K_{3}\right)-4 K_{1}\right)}{32 f_{b}^{2} h^{2} f_{a} f_{c} \rho^{2}} \\
& -\frac{45 f_{a}^{2}}{16 f_{b} f_{c} \rho^{2}}+\frac{9 f_{a}}{8 f_{c} \rho^{2}}+\frac{3 f_{a}}{f_{b} \rho^{2}}+\frac{27 f_{b}}{16 f_{c} \rho^{2}}-\frac{9}{\rho^{2}}+\frac{3 f_{c}}{f_{b} \rho^{2}}+\frac{3 h f_{a} \mu^{2}}{2 f_{1}^{2}}
\end{aligned}
$$

\footnotetext{
${ }^{29}$ See [46] for a recent related discussion in $\mathcal{N}=2^{*}$ model.
} 


$$
\begin{aligned}
& 0=f_{b}^{\prime \prime}-\frac{3 f_{b}}{2 f_{1}^{2}}\left(f_{1}^{\prime}\right)^{2}-\frac{f_{b}}{8 f_{a}^{2}}\left(f_{a}^{\prime}\right)^{2}-\frac{1}{8 f_{b}}\left(f_{b}^{\prime}\right)^{2}+\frac{f_{b}}{8 h^{2}}\left(h^{\prime}\right)^{2}+\frac{f_{b}}{8 g^{2}}\left(g^{\prime}\right)^{2}+\frac{g P^{2}\left(K_{2}^{\prime}\right)^{2}}{36 h f_{a}} \\
& -\frac{3 f_{b}\left(K_{3}^{\prime}\right)^{2}}{32 g h f_{a}^{2} P^{2}}+\frac{5\left(K_{1}^{\prime}\right)^{2}}{32 g h f_{b} P^{2}}+\left(\frac{3 f_{b}^{\prime}}{2 f_{1}}-\frac{3 h^{\prime} f_{b}}{4 h f_{1}}-\frac{3 f_{b} f_{c}^{\prime}}{4 f_{1} f_{c}}-\frac{3 f_{b} f_{a}^{\prime}}{2 f_{a} f_{1}}-\frac{3 f_{b}}{2 \rho f_{1}}\right) f_{1}^{\prime}+\left(\frac{f_{b}^{\prime}}{4 f_{c}}\right. \\
& \left.-\frac{f_{b} f_{a}^{\prime}}{4 f_{a} f_{c}}\right) f_{c}^{\prime}+\frac{f_{b}^{\prime} f_{a}^{\prime}}{2 f_{a}}-\frac{3 f_{b}^{\prime}}{\rho}+\frac{f_{b} h^{\prime}}{h \rho}-\frac{9\left(K_{1}-K_{3}\right)^{2}}{64 h f_{a} g f_{c} \rho^{2} P^{2}}-\frac{K_{1}^{2}}{8 h^{2} f_{a}^{2} f_{b} f_{c} \rho^{2}}+\frac{5 f_{b}}{\rho^{2}}+\frac{27 f_{a}}{16 f_{c} \rho^{2}} \\
& +\frac{g\left(K_{2}^{2}\left(3 f_{a}^{2}-5 f_{b}^{2}\right)+20 f_{b}^{2}\left(K_{2}-1\right)\right) P^{2}}{8 h f_{c} f_{b} f_{a}^{2} \rho^{2}}-\frac{K_{2}\left(K_{1}-K_{3}\right)\left(K_{2}\left(K_{1}-K_{3}\right)-4 K_{1}\right)}{32 f_{c} f_{b} h^{2} f_{a}^{2} \rho^{2}} \\
& +\frac{9 f_{b}}{8 f_{c} \rho^{2}}-\frac{9}{\rho^{2}}-\frac{45 f_{b}^{2}}{16 f_{a} f_{c} \rho^{2}}+\frac{3 f_{b}}{f_{a} \rho^{2}}+\frac{3 f_{c}}{f_{a} \rho^{2}}+\frac{3 h f_{b} \mu^{2}}{2 f_{1}^{2}}, \\
& 0=f_{c}^{\prime \prime}-\frac{f_{c}}{8 f_{a}^{2}}\left(f_{a}^{\prime}\right)^{2}-\frac{f_{c}}{8 f_{b}^{2}}\left(f_{b}^{\prime}\right)^{2}-\frac{1}{2 f_{c}}\left(f_{c}^{\prime}\right)^{2}-\frac{g f_{c} P^{2}\left(K_{2}^{\prime}\right)^{2}}{12 h f_{b} f_{a}}-\frac{3 f_{c}\left(K_{3}^{\prime}\right)^{2}}{32 g h f_{a}^{2} P^{2}}-\frac{3\left(K_{1}^{\prime}\right)^{2} f_{c}}{32 h g f_{b}^{2} P^{2}} \\
& +\frac{f_{c}}{8 g^{2}}\left(g^{\prime}\right)^{2}+\frac{f_{c}}{8 h^{2}}\left(h^{\prime}\right)^{2}-\frac{3 f_{c}}{2 f_{1}^{2}}\left(f_{1}^{\prime}\right)^{2}+\left(\frac{9 f_{c}^{\prime}}{4 f_{1}}-\frac{3 h^{\prime} f_{c}}{4 h f_{1}}-\frac{3 f_{c} f_{b}^{\prime}}{2 f_{b} f_{1}}-\frac{3 f_{c} f_{a}^{\prime}}{2 f_{a} f_{1}}-\frac{3 f_{c}}{2 \rho f_{1}}\right) f_{1}^{\prime} \\
& +\left(\frac{3 f_{b}^{\prime}}{4 f_{b}}+\frac{3 f_{a}^{\prime}}{4 f_{a}}-\frac{3}{\rho}\right) f_{c}^{\prime}-\frac{f_{c} f_{b}^{\prime} f_{a}^{\prime}}{2 f_{b} f_{a}}+\frac{f_{c} h^{\prime}}{h \rho}+\frac{27\left(K_{1}-K_{3}\right)^{2}}{64 h f_{b} f_{a} g \rho^{2} P^{2}}-\frac{K_{1}^{2}}{8 f_{b}^{2} h^{2} f_{a}^{2} \rho^{2}}+\frac{5 f_{c}}{\rho^{2}}+\frac{63 f_{a}}{16 f_{b} \rho^{2}} \\
& +\frac{3 g\left(K_{2}^{2}\left(f_{a}^{2}+f_{b}^{2}\right)-4 f_{b}^{2}\left(K_{2}-1\right)\right) P^{2}}{8 h f_{b}^{2} f_{a}^{2} \rho^{2}}-\frac{K_{2}\left(K_{1}-K_{3}\right)\left(K_{2}\left(K_{1}-K_{3}\right)-4 K_{1}\right)}{32 f_{b}^{2} h^{2} f_{a}^{2} \rho^{2}}-\frac{63}{8 \rho^{2}} \\
& +\frac{3 f_{c}}{f_{b} \rho^{2}}+\frac{63 f_{b}}{16 f_{a} \rho^{2}}+\frac{3 f_{c}}{f_{a} \rho^{2}}-\frac{9 f_{c}^{2}}{f_{a} f_{b} \rho^{2}}+\frac{3 h f_{c} \mu^{2}}{2 f_{1}^{2}} \\
& 0=h^{\prime \prime}-\frac{9}{8 h}\left(h^{\prime}\right)^{2}-\frac{h}{8 g^{2}}\left(g^{\prime}\right)^{2}+\frac{3 h}{2 f_{1}^{2}}\left(f_{1}^{\prime}\right)^{2}+\frac{h}{8 f_{a}^{2}}\left(f_{a}^{\prime}\right)^{2}+\frac{h}{8 f_{b}^{2}}\left(f_{b}^{\prime}\right)^{2}+\frac{g P^{2}\left(K_{2}^{\prime}\right)^{2}}{12 f_{b} f_{a}} \\
& +\frac{3\left(K_{3}^{\prime}\right)^{2}}{32 g f_{a}^{2} P^{2}}+\frac{3\left(K_{1}^{\prime}\right)^{2}}{32 g f_{b}^{2} P^{2}}+\left(\frac{3 f_{b}^{\prime} h}{2 f_{b} f_{1}}+\frac{15 h^{\prime}}{4 f_{1}}+\frac{3 f_{a}^{\prime} h}{2 f_{a} f_{1}}+\frac{3 f_{c}^{\prime} h}{4 f_{1} f_{c}}+\frac{15 h}{2 \rho f_{1}}\right) f_{1}^{\prime}+\left(\frac{h^{\prime}}{2 f_{c}}+\frac{h}{\rho f_{c}}\right. \\
& \left.+\frac{f_{b}^{\prime} h}{4 f_{b} f_{c}}+\frac{f_{a}^{\prime} h}{4 f_{a} f_{c}}\right) f_{c}^{\prime}+\left(\frac{f_{b}^{\prime}}{f_{b}}+\frac{f_{a}^{\prime}}{f_{a}}-\frac{4}{\rho}\right) h^{\prime}+\frac{h f_{b}^{\prime} f_{a}^{\prime}}{2 f_{b} f_{a}}+\frac{2 h f_{b}^{\prime}}{f_{b} \rho}+\frac{2 h f_{a}^{\prime}}{f_{a} \rho}+\frac{45\left(K_{1}-K_{3}\right)^{2}}{64 f_{b} f_{a} g f_{c} \rho^{2} P^{2}} \\
& +\frac{5 g\left(K_{2}^{2}\left(f_{a}^{2}+f_{b}^{2}\right)-4 f_{b}^{2}\left(K_{2}-1\right)\right) P^{2}}{8 f_{b}^{2} f_{a}^{2} f_{c} \rho^{2}}+\frac{9 K_{2}\left(K_{1}-K_{3}\right)\left(K_{2}\left(K_{1}-K_{3}\right)-4 K_{1}\right)}{32 f_{b}^{2} h f_{a}^{2} f_{c} \rho^{2}}-\frac{13 h}{\rho^{2}} \\
& +\frac{9 K_{1}^{2}}{8 f_{b}^{2} h f_{a}^{2} f_{c} \rho^{2}}+\frac{h f_{c}}{f_{a} f_{b} \rho^{2}}-\frac{3 h}{f_{b} \rho^{2}}-\frac{3 h}{f_{a} \rho^{2}}+\frac{9 h f_{a}}{16 f_{b} f_{c} \rho^{2}}-\frac{9 h}{8 f_{c} \rho^{2}}+\frac{9 h f_{b}}{16 f_{a} f_{c} \rho^{2}}-\frac{3 h^{2} \mu^{2}}{2 f_{1}^{2}}, \\
& 0=K_{1}^{\prime \prime}+\left(\frac{f_{c}^{\prime}}{2 f_{c}}-\frac{h^{\prime}}{h}-\frac{f_{b}^{\prime}}{f_{b}}+\frac{f_{a}^{\prime}}{f_{a}}+\frac{3 f_{1}^{\prime}}{f_{1}}-\frac{g^{\prime}}{g}-\frac{3}{\rho}\right) K_{1}^{\prime}-\frac{9 f_{b}\left(K_{1}-K_{3}\right)}{2 f_{a} f_{c} \rho^{2}} \\
& -\frac{g\left(K_{2}\left(K_{2}\left(K_{1}-K_{3}\right)-4 K_{1}+2 K_{3}\right)+4 K_{1}\right) P^{2}}{h f_{a}^{2} f_{c} \rho^{2}}, \\
& 0=K_{2}^{\prime \prime}+\left(\frac{3 f_{1}^{\prime}}{f_{1}}-\frac{h^{\prime}}{h}+\frac{f_{c}^{\prime}}{2 f_{c}}+\frac{g^{\prime}}{g}-\frac{3}{\rho}\right) K_{2}^{\prime}-\frac{9\left(K_{1}-K_{3}\right)\left(K_{2}\left(K_{1}-K_{3}\right)-2 K_{1}\right)}{8 g h f_{a} f_{b} f_{c} P^{2} \rho^{2}} \\
& -\frac{9\left(K_{2}\left(f_{a}^{2}+f_{b}^{2}\right)-2 f_{b}^{2}\right)}{2 f_{a} f_{b} f_{c} \rho^{2}}
\end{aligned}
$$




$$
\begin{aligned}
0= & K_{3}^{\prime \prime}+\left(\frac{f_{c}^{\prime}}{2 f_{c}}-\frac{h^{\prime}}{h}+\frac{f_{b}^{\prime}}{f_{b}}-\frac{f_{a}^{\prime}}{f_{a}}+\frac{3 f_{1}^{\prime}}{f_{1}}-\frac{g^{\prime}}{g}-\frac{3}{\rho}\right) K_{3}^{\prime}+\frac{9 f_{a}\left(K_{1}-K_{3}\right)}{2 f_{b} f_{c} \rho^{2}} \\
& +\frac{K_{2} g\left(K_{2}\left(K_{1}-K_{3}\right)-2 K_{1}\right) P^{2}}{h f_{b}^{2} f_{c} \rho^{2}}, \\
0= & g^{\prime \prime}-\frac{1}{g}\left(g^{\prime}\right)^{2}+\left(\frac{f_{c}^{\prime}}{2 f_{c}}+\frac{f_{b}^{\prime}}{f_{b}}+\frac{f_{a}^{\prime}}{f_{a}}+\frac{3 f_{1}^{\prime}}{f_{1}}-\frac{3}{\rho}\right) g^{\prime}-\frac{P^{2} g^{2}\left(K_{2}^{\prime}\right)^{2}}{9 h f_{a} f_{b}}+\frac{\left(K_{3}^{\prime}\right)^{2}}{8 h f_{a}^{2} P^{2}}+\frac{\left(K_{1}^{\prime}\right)^{2}}{8 h f_{b}^{2} P^{2}} \\
& +\frac{9\left(K_{1}-K_{3}\right)^{2}}{16 f_{a} f_{b} h f_{c} \rho^{2} P^{2}}-\frac{g^{2}\left(K_{2}\left(K_{2}\left(f_{a}^{2}+f_{b}^{2}\right)-4 f_{b}^{2}\right)+4 f_{b}^{2}\right) P^{2}}{2 h f_{a}^{2} f_{b}^{2} f_{c} \rho^{2}} .
\end{aligned}
$$

Additionally we have the first order constraint

$$
\begin{aligned}
0= & \frac{\left(K_{1}^{\prime}\right)^{2}}{P^{2}}+\frac{8 g^{2} P^{2}\left(K_{2}^{\prime}\right)^{2} f_{b}}{9 f_{a}}+\frac{f_{b}^{2}\left(K_{3}^{\prime}\right)^{2}}{f_{a}^{2} P^{2}}+\frac{4 f_{b}^{2} h}{g}\left(g^{\prime}\right)^{2}-\frac{48 h f_{b}^{2} g}{f_{1}^{2}}\left(f_{1}^{\prime}\right)^{2}-8 g f_{b} h\left(\frac{f_{b}^{\prime}}{f_{c}}+\frac{f_{b} f_{a}^{\prime}}{f_{a} f_{c}}\right. \\
& \left.+\frac{3 f_{1}^{\prime} f_{b}}{f_{1} f_{c}}-\frac{4 f_{b}}{\rho f_{c}}\right) f_{c}^{\prime}-24 g f_{b}\left(\frac{2 f_{b}^{\prime} h}{f_{1}}+\frac{h^{\prime} f_{b}}{f_{1}}+\frac{2 f_{a}^{\prime} f_{b} h}{f_{1} f_{a}}-\frac{6 f_{b} h}{f_{1} \rho}\right) f_{1}^{\prime}-4 g h\left(f_{b}^{\prime}\right)^{2} \\
& -\frac{16 g h f_{b}^{\prime} f_{a}^{\prime} f_{b}}{f_{a}}+\frac{4 g\left(h^{\prime}\right)^{2} f_{b}^{2}}{h}-\frac{4 g h\left(f_{a}^{\prime}\right)^{2} f_{b}^{2}}{f_{a}^{2}}+\frac{64 g h f_{b}^{\prime} f_{b}}{\rho}+\frac{32 g h^{\prime} f_{b}^{2}}{\rho}+\frac{64 g h f_{a}^{\prime} f_{b}^{2}}{\rho f_{a}} \\
& -\frac{9 f_{b}\left(K_{1}-K_{3}\right)^{2}}{2 f_{a} \rho^{2} f_{c} P^{2}}-\frac{4 g^{2}\left(K_{2}^{2}\left(f_{a}^{2}+f_{b}^{2}\right)-4 f_{b}^{2}\left(K_{2}-1\right)\right) P^{2}}{\rho^{2} f_{c} f_{a}^{2}}-\frac{4 g K_{1}^{2}}{h \rho^{2} f_{c} f_{a}^{2}} \\
& -\frac{g K_{2}\left(K_{1}-K_{3}\right)\left(K_{2}\left(K_{1}-K_{3}\right)-4 K_{1}\right)}{h \rho^{2} f_{c} f_{a}^{2}}-2 g f_{b} h\left(\frac{48 f_{b}}{\rho^{2}}+\frac{16 f_{c}}{f_{a} \rho^{2}}-\frac{48}{\rho^{2}}-\frac{48 f_{b}}{f_{a} \rho^{2}}+\frac{9 f_{a}}{f_{c} \rho^{2}}\right. \\
& \left.-\frac{18 f_{b}}{f_{c} \rho^{2}}+\frac{9 f_{b}^{2}}{f_{a} f_{c} \rho^{2}}\right)+\frac{48 h^{2} f_{b}^{2} g \mu^{2}}{f_{1}^{2}} .
\end{aligned}
$$

We explicitly verified that the constraint (A.10) is consistent with (A.1)-(A.9). Thus the second-order equation for $f_{c}$ (A.4) can be eliminated in favor of the constraint equation (A.10) where $f_{c}^{\prime}$ enters linearly. In total, we expect that a solution is specified by $8 \times 2+1 \times 1=17$ parameters ( 8 second-order equations for $f_{a, b}, K_{1,2,3}, h, g, f_{1}$ and a single first-order equation for $f_{c}$ ).

The general UV (as $\rho \rightarrow 0$ ) asymptotic solution of (A.1)-(A.10) describing the vacua of the warped deformed conifold with fluxes and $S^{3}$ spatial boundary, i.e., the vacua of the cascading gauge theory on $S^{3}$, takes the form

$$
\begin{aligned}
f_{a}= & +f_{a, 1,0} \rho+\rho^{2}\left(\frac{5}{16} \mu^{2} P^{2} g_{s}+\frac{1}{4} \mu^{2} K_{0}+\frac{1}{4} f_{a, 1,0}^{2}-\frac{1}{2} \mu^{2} P^{2} g_{s} \ln \rho\right)+\rho^{3} f_{a, 3,0} \\
& +\mathcal{O}\left(\rho^{4} \ln ^{2} \rho\right), \\
f_{b}= & +f_{a, 1,0} \rho+\rho^{2}\left(\frac{5}{16} \mu^{2} P^{2} g_{s}+\frac{1}{4} \mu^{2} K_{0}+\frac{1}{4} f_{a, 1,0}^{2}-\frac{1}{2} \mu^{2} P^{2} g_{s} \ln \rho\right) \\
& +\rho^{3}\left(\frac{1}{2} \mu^{2} f_{a, 1,0} P^{2} g_{s}-f_{a, 3,0}\right)+\mathcal{O}\left(\rho^{4} \ln ^{2} \rho\right), \\
f_{c}= & +f_{a, 1,0} \rho+\rho^{2}\left(\frac{3}{8} \mu^{2} P^{2} g_{s}+\frac{1}{4} \mu^{2} K_{0}+\frac{1}{4} f_{a, 1,0}^{2}-\frac{1}{2} \mu^{2} P^{2} g_{s} \ln \rho\right)+\frac{1}{4} \mu^{2} f_{a, 1,0} P^{2} g_{s} \rho^{3} \\
& +\mathcal{O}\left(\rho^{4} \ln ^{2} \rho\right),
\end{aligned}
$$




$$
\begin{aligned}
h= & \frac{1}{8} P^{2} g_{s}+\frac{1}{4} K_{0}-\frac{1}{2} P^{2} g_{s} \ln \rho+\rho\left(-\frac{1}{2} f_{a, 1,0} K_{0}+f_{a, 1,0} P^{2} g_{s} \ln \rho\right)+\rho^{2}\left(\frac{23}{288} \mu^{2} P^{4} g_{s}^{2}\right. \\
& -\frac{1}{6} P^{2} g_{s} \mu^{2} K_{0}-\frac{1}{4} P^{2} g_{s} f_{a, 1,0}^{2}-\frac{1}{8} \mu^{2} K_{0}^{2}+\frac{5}{8} f_{a, 1,0}^{2} K_{0}+\frac{1}{96} P^{2} g_{s}\left(32 P^{2} g_{s} \mu^{2}+48 K_{0} \mu^{2}\right. \\
& \left.\left.-120 f_{a, 1,0}^{2}\right) \ln \rho-\frac{1}{2} \mu^{2} P^{4} g_{s}^{2} \ln ^{2} \rho\right)+\rho^{3}\left(\frac{1}{4} P^{2} g_{s} \mu^{2} f_{a, 1,0} K_{0}-\frac{13}{32} P^{4} g_{s}^{2} f_{a, 1,0} \mu^{2}\right. \\
& +\frac{11}{24} P^{2} g_{s} f_{a, 1,0}^{3}+\frac{3}{8} \mu^{2} f_{a, 1,0} K_{0}^{2}-\frac{5}{8} K_{0} f_{a, 1,0}^{3}+\left(\frac{5}{4} P^{2} g_{s} f_{a, 1,0}^{3}-\frac{1}{2} P^{4} g_{s}^{2} f_{a, 1,0} \mu^{2}\right. \\
& \left.\left.-\frac{3}{2} P^{2} g_{s} \mu^{2} f_{a, 1,0} K_{0}\right) \ln \rho+\frac{3}{2} P^{4} g_{s}^{2} f_{a, 1,0} \mu^{2} \ln ^{2} \rho\right)+\mathcal{O}\left(\rho^{4} \ln ^{3} \rho\right), \\
K_{1}= & K_{0}-2 P^{2} g_{s} \ln \rho+P^{2} g_{s} f_{a, 1,0} \rho+\rho^{2}\left(\frac{1}{4} P^{2} g_{s}\left(3 P^{2} g_{s} \mu^{2}+K_{0} \mu^{2}-f_{a, 1,0}^{2}\right)\right. \\
& \left.-\frac{1}{2} \mu^{2} P^{4} g_{s}^{2} \ln \rho\right)+\rho^{3}\left(\frac{1}{12} P^{2} g_{s}\left(8 k_{2,3,0}-8 P^{2} f_{a, 1,0} g_{s} \mu^{2}-3 K_{0} f_{a, 1,0} \mu^{2}+f_{a, 1,0}^{3}+8 f_{a, 3,0}\right)\right. \\
& \left.+2 P^{2} g_{s} f_{a, 3,0} \ln \rho\right)+\mathcal{O}\left(\rho^{4} \ln ^{2} \rho\right), \\
K_{2}= & +\rho^{3}\left(k_{2,3,0}+\left(3 f_{a, 3,0}-\frac{3}{4} \mu^{2} f_{a, 1,0} P^{2} g_{s}\right) \ln \rho\right)+\mathcal{O}\left(\rho^{4} \ln \rho\right), \\
K_{3}= & K_{0}-2 P^{2} g_{s} \ln \rho+P^{2} g_{s} f_{a, 1,0} \rho+\rho^{2}\left(\frac{1}{4} P^{2} g_{s}\left(3 P^{2} g_{s} \mu^{2}+K_{0} \mu^{2}-f_{a, 1,0}^{2}\right)\right. \\
& \left.-\frac{1}{2} \mu^{2} P^{4} g_{s}^{2} \ln \rho\right)+\rho^{3}\left(\frac{1}{12} P^{2} g_{s}\left(f_{a, 1,0}^{3}-4 P^{2} g_{s} f_{a, 1,0} \mu^{2}-3 K_{0} f_{a, 1,0} \mu^{2}-8 f_{a, 3,0}-8 k_{2,3,0}\right)\right. \\
& \left.+P^{2} g_{s}\left(P^{2} g_{s} f_{a, 1,0} \mu^{2}-2 f_{a, 3,0}\right) \ln \rho\right)+\mathcal{O}\left(\rho^{4} \ln ^{2} \rho\right), \\
g= & g_{s}\left(1-\frac{1}{4} \mu^{2} P^{2} g_{s} \rho^{2}+\frac{1}{4} \mu^{2} f_{a, 1,0} P^{2} g_{s} \rho^{3}+\mathcal{O}\left(\rho^{4} \ln \rho\right)\right), \\
f_{1}= & +\rho^{2}\left(-\frac{1}{16} \mu^{2} P^{2} g_{s}-\frac{1}{8} \mu^{2} K_{0}+\frac{1}{4} \mu^{2} P^{2} g_{s} \ln \rho\right)+\rho_{0}\left(-\frac{1}{16} \mu^{2} f_{a, 1,0} P^{2} g_{s}\right. \\
&
\end{aligned}
$$

It is characterized by the cascading gauge theory defining parameters

$$
K_{0}, \mu, P, g_{s},
$$

correspondingly related to the strong coupling scale $\Lambda$ (2.48), the $S^{3}$ compactification scale $\mu$ (3.16), the rank difference of the gauge group factors $M(3.6)$, and the renormalization group flow invariant sum of the gauge couplings (2.28). Additionally, there are 9 normalizable coefficients related to the diffeomorphism parameter $\alpha$ (3.17) and the expectation values of the various operators in the boundary theory

$$
\left\{f_{a, 1,0}, f_{a, 3,0}, k_{2,3,0}, f_{a, 4,0}, f_{c, 4,0}, g_{4,0}, f_{a, 6,0}, k_{2,7,0}, f_{a, 8,0}\right\} .
$$

The IR (as $y \equiv \frac{1}{\rho} \rightarrow 0$ ) asymptotics of (A.1)-(A.10) differ depending on the topology of the background geometry. We call vacua of the cascading gauge theory with the boundary 
spatial $S^{3}$ smoothly shrinking to zero size $\mathcal{V}_{A}$, and vacua with the 2-cycle of the warped deformed conifold smoothly shrinking to zero size $\mathcal{V}_{B}$. Note that the 3 -cycle of the warped deformed conifold can not vanish without producing a naked singularity since it supports nonzero (when $P \neq 0$ ) RR 3-form flux (3.5).

- $\mathcal{V}_{A}$ vacua of the cascading gauge theory. To identify smooth geometries with vanishing $S^{3}$ as $y \rightarrow 0$ we introduce

$$
f_{1}^{h} \equiv y^{-1} f_{1}, \quad f_{a, b, c}^{h} \equiv y^{2} f_{a, b, c}, \quad h^{h} \equiv y^{-4} h .
$$

The IR asymptotic expansion

$$
\begin{aligned}
f_{a, b, c}^{h} & =\sum_{n=0} f_{a, b, c, n}^{h} y^{2 n}, & h^{h} & =h_{0}^{h}+\sum_{n=1} h_{n}^{h} y^{2 n}, \\
g & =\sum_{n=0} g_{n}^{h} y^{2 n}, & f_{1}^{h} & =\mu \sqrt{h_{0}^{h}}\left(1+\sum_{n=1} f_{1, n}^{h} y^{2 n}\right),
\end{aligned}
$$

is characterized by 8 parameters:

$$
\left\{f_{a, 0}^{h}, f_{b, 0}^{h}, f_{c, 0}^{h}, h_{0}^{h}, K_{1,0}^{h}, K_{2,0}^{h}, K_{3,0}^{h}, g_{0}^{h}\right\} .
$$

Note that given (A.23),

$$
\begin{aligned}
& \frac{1}{h^{1 / 2} \rho^{2}}\left(-d t^{2}+\frac{f_{1}^{2}}{\mu^{2}}\left(d S^{3}\right)^{2}\right)+\frac{h^{1 / 2}}{\rho^{2}}(d \rho)^{2}=\frac{1}{\sqrt{h^{h}}}\left(-d t^{2}+\frac{\left(f_{1}^{h}\right)^{2}}{\mu^{2}} y^{2}\left(d S^{3}\right)^{2}\right) \\
& +\sqrt{h^{h}}(d y)^{2} \underbrace{\longrightarrow}_{y \rightarrow 0}-\frac{1}{\sqrt{h_{0}^{h}}} d t^{2}+\sqrt{h_{0}^{h}}\left(y^{2}\left(d S^{3}\right)^{2}+(d y)^{2}\right),
\end{aligned}
$$

i.e., $S^{3}$ indeed smoothly shrinks to zero size as $y \rightarrow 0$. It is important to emphasize that $\mathcal{V}_{A}$ vacua defined by (A.23) have either $\mathrm{U}(1)$ or $\mathbb{Z}_{2}$ chiral symmetry - the chiral symmetry is unbroken in the former $\left(\mathcal{V}_{A}^{s}\right.$ vacua), and is spontaneously broken in the latter $\left(\mathcal{V}_{A}^{b}\right.$ vacua). Specifically, unbroken chiral symmetry dictates (3.28), leading to

$$
\begin{aligned}
& \mathrm{UV}: f_{a, 3,0}=\frac{1}{4} \mu^{2} f_{a, 1,0} P^{2} g_{s}, \quad k_{2,3,0}=0, \quad k_{2,7,0}=0 ; \\
& \text { IR : } f_{b, 0}^{h}=f_{a, 0}^{h}, \quad K_{3,0}^{h}=K_{1,0}^{h}, \quad K_{2,0}^{h}=1 .
\end{aligned}
$$

- $\mathcal{V}_{B}$ vacua of the cascading gauge theory. To identify smooth geometries with vanishing $S^{2}$ as $y \rightarrow 0$ we introduce

$$
f_{a, b, c}^{h} \equiv y^{2} f_{a, b, c}, \quad h^{h} \equiv y^{-4} h .
$$

The IR asymptotic expansion

$$
\begin{aligned}
f_{a}^{h} & =f_{a, 0}^{h}+\sum_{n=1} f_{a, n} y^{2 n}, & f_{b}^{h} & =3 y^{2}+\sum_{n=2} f_{b, n} y^{2 n}, \quad f_{c}^{h}=\frac{3}{4} f_{a, 0}^{h}+\sum_{n=1} f_{c, n} y^{2 n}, \\
K_{1} & =K_{1,3}^{h} y^{3}+\sum_{n=2} K_{1, n} y^{2 n+1}, & K_{2} & =K_{2,2}^{h} y^{2}+\sum_{n=2} K_{2, n} y^{2 n} \\
K_{3} & =K_{3,1}^{h} y+\sum_{n=1} K_{3, n} y^{2 n+1}, & h^{h} & =h_{0}^{h}+\sum_{n=1} h_{n}^{h} y^{2 n} \\
g & =\sum_{n=0} g_{n}^{h} y^{2 n}, & f_{1}^{h} & =\sum_{n=0} f_{1, n}^{h} y^{2 n}
\end{aligned}
$$


is characterized by 8 parameters:

$$
\left\{f_{a, 0}^{h}, h_{0}^{h}, K_{1,3}^{h}, K_{2,2}^{h}, K_{2,4}^{h}, K_{3,1}^{h}, g_{0}^{h}, f_{1,0}^{h}\right\} .
$$

Note that given (A.28),

$$
\begin{aligned}
& \frac{h^{1 / 2}}{\rho^{2}}(d \rho)^{2}+\frac{f_{b} h^{1 / 2}}{6}\left(g_{1}^{2}+g_{2}^{2}\right)=\sqrt{h^{h}}(d y)^{2}+\left.\frac{f_{b}^{h}\left(h^{h}\right)^{1 / 2}}{3} \frac{1}{2}\left(g_{1}^{2}+g_{2}^{2}\right)\right|_{2-\text { cycle }} \\
& \underbrace{\longrightarrow}_{y \rightarrow 0} \sqrt{h_{0}^{h}}\left(y^{2}\left(d S^{2}\right)^{2}+(d y)^{2}\right)
\end{aligned}
$$

where $\left.\right|_{2-\text { cycle }}$ means restriction to the $T^{1,1}$ 2-cycle. Following [13], this means setting $\psi=0, \phi_{2}=-\phi_{1}, \theta_{2}=-\theta_{1}$ in one-forms $\left\{g_{i}\right\}$ (see $\left.(2.21)\right)$ on $T^{1,1}$ :

$$
\left.\left(g_{1}^{2}+g_{2}^{2}\right)\right|_{2-\text { cycle }}=2\left(\left(d \theta_{1}\right)^{2}+\sin ^{2} \theta_{1}\left(d \phi_{1}\right)^{2}\right)=2\left(d S^{2}\right)^{2}
$$

On the other hand, the 3 -cycle supporting RR flux remains finite, provided $f_{a, 0}^{h} h_{0}^{h} \neq 0$ :

$$
\begin{aligned}
& \frac{f_{c} h^{1 / 2}}{9} g_{5}^{2}+\frac{f_{a} h^{1 / 2}}{6}\left(g_{3}^{2}+g_{4}^{2}\right)=\frac{f_{c}^{h}\left(h^{h}\right)^{1 / 2}}{9} g_{5}^{2}+\frac{f_{a}^{h}\left(h^{h}\right)^{1 / 2}}{6}\left(g_{3}^{2}+g_{4}^{2}\right) \\
& \left.\underbrace{\longrightarrow}_{y \rightarrow 0} \quad \frac{f_{a, 0}^{h}\left(h_{0}^{h}\right)^{1 / 2}}{6}\left(\frac{1}{2} g_{5}^{2}+g_{3}^{2}+g_{4}^{2}\right)\right|_{3 \text {-cycle: } \theta_{2}=\phi_{2}=0, \theta_{1}=2 \eta, \psi=\xi_{1}+\xi_{2}, \phi_{1}=\xi_{1}-\xi_{2}} \\
& =\frac{f_{a, 0}^{h}\left(h_{0}^{h}\right)^{1 / 2}}{6} 2\left((d \eta)^{2}+\cos ^{2} \eta\left(d \xi_{1}\right)^{2}+\sin ^{2} \eta\left(d \xi_{2}\right)^{2}\right)=\frac{f_{a, 0}^{h}\left(h_{0}^{h}\right)^{1 / 2}}{3}\left(d S^{3}\right)^{2} .
\end{aligned}
$$

From (A.30), $S^{2}$ indeed smoothly shrinks to zero size as $y \rightarrow 0$. Because $f_{a} \neq f_{b}$ as $y \rightarrow 0, \mathcal{V}_{B}$ vacua defined by (A.28) have only $\mathbb{Z}_{2}$ chiral symmetry - the chiral symmetry is spontaneously broken.

\section{A.1.1 Klebanov-Strassler solution [2] as $\mu \rightarrow 0$ limit of $\mathcal{V}_{B}$ conifold vacua}

The supersymmetric Klebanov-Strassler solution [2] is a decompactification limit $\mu \rightarrow 0$ of $\mathcal{V}_{B}$ conifold vacua discussed above (see section 2 for a review). Indeed,

- in the UV, using (2.43) we identify (see (A.21)):

$$
\begin{aligned}
& f_{a, 1,0}=-2 \mathcal{Q}, \quad f_{a, 3,0}=\frac{3 \sqrt{6}}{4} \epsilon^{2}, \quad k_{2,3,0}=\frac{3 \sqrt{6}}{8} \epsilon^{2}(3 \ln 3-5 \ln 2+4 \ln \epsilon), \\
& f_{a, 4,0}=\frac{3 \sqrt{6}}{4} \mathcal{Q} \epsilon^{2}, \quad f_{c, 4,0}=0, \quad g_{4,0}=0 \\
& f_{a, 6,0}=\left(-\frac{27}{16} \ln 2+\frac{81}{50}+\frac{81}{80} \ln 3+\frac{27}{20} \ln \epsilon\right) \epsilon^{4}+\frac{3 \sqrt{6}}{4} \mathcal{Q}^{3} \epsilon^{2} \\
& k_{2,7,0}=\frac{45 \sqrt{6}}{8}\left(\frac{57}{10}-5 \ln 2+3 \ln 3+4 \ln \epsilon\right) \mathcal{Q}^{4} \epsilon^{2} \\
& f_{a, 8,0}=\left(\frac{27}{2} \ln \epsilon-\frac{135}{8} \ln 2+\frac{81}{8} \ln 3+\frac{405}{16}\right) \mathcal{Q}^{2} \epsilon^{4}+\frac{3 \sqrt{6}}{4} \mathcal{Q}^{5} \epsilon^{2}
\end{aligned}
$$


- in the IR, using (2.45) we identify (see (A.29)):

$$
\begin{aligned}
& f_{a, 0}^{h}=2^{1 / 3} 3^{2 / 3} \epsilon^{4 / 3}, \quad h_{0}^{h}=P^{2} g_{s} \epsilon^{-8 / 3} \times 0.056288(0), \\
& K_{1,3}^{h}=\frac{4 \sqrt{6}}{9 \epsilon^{2}} P^{2} g_{s}, \quad K_{2,2}^{h}=\frac{2^{2 / 3}}{3^{2 / 3} \epsilon^{4 / 3}}, \quad K_{2,4}^{h}=-\frac{112^{1 / 3} 3^{2 / 3}}{45 \epsilon^{8 / 3}}, \\
& K_{3,1}^{h}=\frac{4 \sqrt{6} 2^{1 / 3} 3^{2 / 3}}{27 \epsilon^{2 / 3}} P^{2} g_{s}, \quad g_{0}^{h}=g_{s}, \quad f_{1,0}^{h}=1 .
\end{aligned}
$$

The relation between $K_{0}$ and the strong coupling scale $\Lambda$ of the cascading gauge theory (2.39) is given by (2.48):

$$
K_{0}=P^{2} g_{s}\left(\frac{5}{3} \ln 2-\ln 3-\frac{2}{3}-\frac{4}{3} \ln \epsilon\right) .
$$

\section{A.2 Case (B): schwarzschild horizon in warped deformed conifold with fluxes}

Using the metric ansatz (3.19) we derive from (3.1) the following equations of motion:

$$
\begin{aligned}
& 0=f^{\prime \prime}-\frac{f^{\prime}}{2}\left(\frac{6}{\rho}-\frac{2 f_{b}^{\prime}}{f_{b}}-\frac{2 f_{a}^{\prime}}{f_{a}}-\frac{f_{c}^{\prime}}{f_{c}}\right)+4 h \mu^{2}, \\
& 0=f_{a}^{\prime \prime}-\frac{1}{8 f_{a}}\left(f_{a}^{\prime}\right)^{2}-\frac{f_{a}}{8 f_{b}^{2}}\left(f_{b}^{\prime}\right)^{2}+\frac{f_{a}}{8 g^{2}}\left(g^{\prime}\right)^{2}+\frac{f_{a}}{8 h^{2}}\left(h^{\prime}\right)^{2}+\frac{g P^{2}\left(K_{2}^{\prime}\right)^{2}}{36 h f_{b}}-\frac{3 f_{a}\left(K_{1}^{\prime}\right)^{2}}{32 g h f_{b}^{2} P^{2}} \\
& +\frac{5\left(K_{3}^{\prime}\right)^{2}}{32 g h f_{a} P^{2}}+\left(\frac{3 f_{a}^{\prime}}{4 f}-\frac{f_{a} h^{\prime}}{8 h f}-\frac{f_{a} f_{b}^{\prime}}{4 f_{b} f}-\frac{f_{a} f_{c}^{\prime}}{8 f_{c} f}-\frac{5 f_{a}}{4 \rho f}\right) f^{\prime}+\left(\frac{f_{a}^{\prime}}{4 f_{c}}-\frac{f_{a} f_{b}^{\prime}}{4 f_{b} f_{c}}\right) f_{c}^{\prime}+\frac{f_{b}^{\prime} f_{a}^{\prime}}{2 f_{b}} \\
& +\frac{f_{a} h^{\prime}}{h \rho}-\frac{3 f_{a}^{\prime}}{\rho}-\frac{9\left(K_{3}-K_{1}\right)^{2}}{64 h f_{b} g f_{c} \rho^{2} f P^{2}}-\frac{g\left(K_{2}^{2}\left(5 f_{a}^{2}-3 f_{b}^{2}\right)+12 f_{b}^{2}\left(K_{2}-1\right)\right) P^{2}}{8 h f_{b}^{2} f_{a} f_{c} \rho^{2} f} \\
& -\frac{K_{2}\left(K_{3}-K_{1}\right)\left(K_{2}\left(K_{3}-K_{1}\right)+4 K_{1}\right)}{32 f_{b}^{2} h^{2} f_{a} f_{c} \rho^{2} f}-\frac{K_{1}^{2}}{8 f_{b}^{2} h^{2} f_{a} f_{c} \rho^{2} f}+\frac{5 f_{a}}{\rho^{2}}-\frac{45 f_{a}^{2}}{16 f_{b} f_{c} \rho^{2} f}+\frac{9 f_{a}}{8 f_{c} \rho^{2} f} \\
& +\frac{3 f_{a}}{f_{b} \rho^{2} f}+\frac{27 f_{b}}{16 f_{c} \rho^{2} f}-\frac{9}{\rho^{2} f}+\frac{3 f_{c}}{f_{b} \rho^{2} f}-\frac{5 h f_{a} \mu^{2}}{2 f}, \\
& 0=f_{b}^{\prime \prime}-\frac{f_{b}\left(f_{a}^{\prime}\right)^{2}}{8 f_{a}^{2}}-\frac{\left(f_{b}^{\prime}\right)^{2}}{8 f_{b}}+\frac{f_{b}\left(h^{\prime}\right)^{2}}{8 h^{2}}-\frac{3 f_{b}\left(K_{3}^{\prime}\right)^{2}}{32 h f_{a}^{2} P^{2} g}+\frac{5\left(K_{1}^{\prime}\right)^{2}}{32 f_{b} h P^{2} g}+\frac{P^{2} g\left(K_{2}^{\prime}\right)^{2}}{36 h f_{a}}+\frac{f_{b}\left(g^{\prime}\right)^{2}}{8 g^{2}} \\
& +\left(\frac{3 f_{b}^{\prime}}{4 f}-\frac{f_{b} f_{a}^{\prime}}{4 f_{a} f}-\frac{f_{b} h^{\prime}}{8 h f}-\frac{5 f_{b}}{4 \rho f}-\frac{f_{b} f_{c}^{\prime}}{8 f_{c} f}\right) f^{\prime}+\left(\frac{f_{b}^{\prime}}{4 f_{c}}-\frac{f_{b} f_{a}^{\prime}}{4 f_{c} f_{a}}\right) f_{c}^{\prime}+\frac{f_{b}^{\prime} f_{a}^{\prime}}{2 f_{a}}-\frac{3 f_{b}^{\prime}}{\rho}+\frac{f_{b} h^{\prime}}{h \rho} \\
& +\frac{g\left(K_{2}^{2}\left(3 f_{a}^{2}-5 f_{b}^{2}\right)+20 f_{b}^{2}\left(K_{2}-1\right)\right) P^{2}}{8 h f_{c} f_{b} f_{a}^{2} \rho^{2} f}-\frac{K_{2}\left(K_{3}-K_{1}\right)\left(K_{2}\left(K_{3}-K_{1}\right)+4 K_{1}\right)}{32 f_{c} f_{b} h^{2} f_{a}^{2} \rho^{2} f} \\
& -\frac{9\left(K_{3}-K_{1}\right)^{2}}{64 h f_{a} g f_{c} \rho^{2} f P^{2}}-\frac{K_{1}^{2}}{8 f_{c} f_{b} h^{2} f_{a}^{2} \rho^{2} f}+\frac{5 f_{b}}{\rho^{2}}+\frac{27 f_{a}}{16 f_{c} \rho^{2} f}+\frac{9 f_{b}}{8 f_{c} \rho^{2} f}-\frac{9}{\rho^{2} f}-\frac{45 f_{b}^{2}}{16 f_{a} f_{c} \rho^{2} f} \\
& +\frac{3 f_{b}}{f_{a} \rho^{2} f}+\frac{3 f_{c}}{f_{a} \rho^{2} f}-\frac{5 h f_{b} \mu^{2}}{2 f}, \\
& 0=f_{c}^{\prime \prime}-\frac{\left(f_{c}^{\prime}\right)^{2}}{2 f_{c}}-\frac{f_{c}\left(f_{a}^{\prime}\right)^{2}}{8 f_{a}^{2}}-\frac{f_{c}\left(f_{b}^{\prime}\right)^{2}}{8 f_{b}^{2}}+\frac{f_{c}\left(h^{\prime}\right)^{2}}{8 h^{2}}-\frac{P^{2} g f_{c}\left(K_{2}^{\prime}\right)^{2}}{12 f_{b} h f_{a}}-\frac{3 f_{c}\left(K_{3}^{\prime}\right)^{2}}{32 h f_{a}^{2} P^{2} g}-\frac{3 f_{c}\left(K_{1}^{\prime}\right)^{2}}{32 f_{b}^{2} h P^{2} g} \\
& +\frac{f_{c}\left(g^{\prime}\right)^{2}}{8 g^{2}}+\left(\frac{7 f_{c}^{\prime}}{8 f}-\frac{f_{c} f_{b}^{\prime}}{4 f_{b} f}-\frac{h^{\prime} f_{c}}{8 h f}-\frac{f_{c} f_{a}^{\prime}}{4 f_{a} f}-\frac{5 f_{c}}{4 \rho f}\right) f^{\prime}+\left(\frac{3 f_{b}^{\prime}}{4 f_{b}}+\frac{3 f_{a}^{\prime}}{4 f_{a}}-\frac{3}{\rho}\right) f_{c}^{\prime}-\frac{f_{c} f_{b}^{\prime} f_{a}^{\prime}}{2 f_{b} f_{a}} \\
& +\frac{f_{c} h^{\prime}}{h \rho}+\frac{27\left(K_{3}-K_{1}\right)^{2}}{64 f_{b} h f_{a} g \rho^{2} f P^{2}}+\frac{3 g\left(K_{2}^{2}\left(f_{a}^{2}+f_{b}^{2}\right)-4 f_{b}^{2}\left(K_{2}-1\right)\right) P^{2}}{8 h f_{b}^{2} f_{a}^{2} \rho^{2} f}-\frac{K_{1}^{2}}{8 f_{b}^{2} h^{2} f_{a}^{2} \rho^{2} f}+\frac{5 f_{c}}{\rho^{2}}
\end{aligned}
$$




$$
\begin{aligned}
& +\frac{63 f_{a}}{16 f_{b} \rho^{2} f}-\frac{K_{2}\left(K_{3}-K_{1}\right)\left(K_{2}\left(K_{3}-K_{1}\right)+4 K_{1}\right)}{32 f_{b}^{2} h^{2} f_{a}^{2} \rho^{2} f}-\frac{63}{8 \rho^{2} f}+\frac{3 f_{c}}{f_{b} \rho^{2} f}+\frac{63 f_{b}}{16 f_{a} \rho^{2} f} \\
& +\frac{3 f_{c}}{f_{a} \rho^{2} f}-\frac{9 f_{c}^{2}}{f_{a} f_{b} \rho^{2} f}-\frac{5 h f_{c} \mu^{2}}{2 f}, \\
& 0=h^{\prime \prime}+\frac{g P^{2}\left(K_{2}^{\prime}\right)^{2}}{12 f_{b} f_{a}}+\frac{3\left(K_{1}^{\prime}\right)^{2}}{32 g f_{b}^{2} P^{2}}+\frac{3\left(K_{3}^{\prime}\right)^{2}}{32 g f_{a}^{2} P^{2}}-\frac{h}{8 g^{2}}\left(g^{\prime}\right)^{2}-\frac{9}{8 h}\left(h^{\prime}\right)^{2}+\frac{h}{8 f_{a}^{2}}\left(f_{a}^{\prime}\right)^{2} \\
& +\frac{h}{8 f_{b}^{2}}\left(f_{b}^{\prime}\right)^{2}+\left(\frac{h f_{b}^{\prime}}{4 f_{b} f}+\frac{9 h^{\prime}}{8 f}+\frac{h f_{a}^{\prime}}{4 f_{a} f}+\frac{13 h}{4 \rho f}+\frac{h f_{c}^{\prime}}{8 f_{c} f}\right) f^{\prime}+\left(\frac{h f_{b}^{\prime}}{4 f_{c} f_{b}}+\frac{h^{\prime}}{2 f_{c}}+\frac{h f_{a}^{\prime}}{4 f_{c} f_{a}}\right. \\
& \left.+\frac{h}{f_{c} \rho}\right) f_{c}^{\prime}+\left(\frac{f_{a}^{\prime}}{f_{a}}+\frac{f_{b}^{\prime}}{f_{b}}-\frac{4}{\rho}\right) h^{\prime}+\frac{h f_{b}^{\prime} f_{a}^{\prime}}{2 f_{a} f_{b}}+\frac{2 h f_{b}^{\prime}}{f_{b} \rho}+\frac{2 h f_{a}^{\prime}}{f_{a} \rho}+\frac{45\left(K_{3}-K_{1}\right)^{2}}{64 f_{b} f_{a} g f_{c} \rho^{2} f P^{2}}-\frac{13 h}{\rho^{2}} \\
& +\frac{9 K_{1}^{2}}{8 f_{c} f_{b}^{2} h f_{a}^{2} \rho^{2} f}+\frac{5 g\left(K_{2}^{2}\left(f_{a}^{2}+f_{b}^{2}\right)-4 f_{b}^{2}\left(K_{2}-1\right)\right) P^{2}}{8 f_{c} f_{b}^{2} f_{a}^{2} \rho^{2} f}+\frac{9 K_{1} K_{2}\left(K_{3}-K_{1}\right)}{8 f_{c} f_{b}^{2} h f_{a}^{2} \rho^{2} f}+\frac{h f_{c}}{f_{a} f_{b} \rho^{2} f} \\
& +\frac{9 f_{a} h}{16 f_{b} f_{c} \rho^{2} f}+\frac{9\left(K_{3}-K_{1}\right)^{2} K_{2}^{2}}{32 f_{c} f_{b}^{2} h f_{a}^{2} \rho^{2} f}-\frac{9 h}{8 f_{c} \rho^{2} f}-\frac{3 h}{f_{b} \rho^{2} f}+\frac{9 h f_{b}}{16 f_{a} f_{c} \rho^{2} f}-\frac{3 h}{f_{a} \rho^{2} f}+\frac{13 h^{2} \mu^{2}}{2 f}, \\
& 0=K_{1}^{\prime \prime}+\left(\frac{f^{\prime}}{f}+\frac{f_{c}^{\prime}}{2 f_{c}}-\frac{f_{b}^{\prime}}{f_{b}}-\frac{h^{\prime}}{h}-\frac{g^{\prime}}{g}+\frac{f_{a}^{\prime}}{f_{a}}-\frac{3}{\rho}\right) K_{1}^{\prime}+\frac{9 f_{b}\left(K_{3}-K_{1}\right)}{2 f_{a} f f_{c} \rho^{2}} \\
& +\frac{g\left(K_{2}\left(K_{2}\left(K_{3}-K_{1}\right)-2 K_{3}\right)+4 K_{1}\left(K_{2}-1\right)\right) P^{2}}{h f f_{c} f_{a}^{2} \rho^{2}}, \\
& 0=K_{2}^{\prime \prime}+\left(\frac{g^{\prime}}{g}+\frac{f_{c}^{\prime}}{2 f_{c}}-\frac{h^{\prime}}{h}+\frac{f^{\prime}}{f}-\frac{3}{\rho}\right) K_{2}^{\prime}-\frac{9\left(K_{3}-K_{1}\right)\left(K_{2}\left(K_{3}-K_{1}\right)+2 K_{1}\right)}{8 h f f_{c} f_{a} f_{b} g P^{2} \rho^{2}} \\
& -\frac{9\left(\left(f_{a}^{2}+f_{b}^{2}\right) K_{2}-2 f_{b}^{2}\right)}{2 f f_{c} f_{a} f_{b} \rho^{2}}, \\
& 0=K_{3}^{\prime \prime}+\left(\frac{f^{\prime}}{f}-\frac{g^{\prime}}{g}+\frac{f_{b}^{\prime}}{f_{b}}-\frac{f_{a}^{\prime}}{f_{a}}+\frac{f_{c}^{\prime}}{2 f_{c}}-\frac{h^{\prime}}{h}-\frac{3}{\rho}\right) K_{3}^{\prime}-\frac{9 f_{a}\left(K_{3}-K_{1}\right)}{2 f_{b} f f_{c} \rho^{2}} \\
& -\frac{g K_{2}\left(K_{2}\left(K_{3}-K_{1}\right)+2 K_{1}\right) P^{2}}{h f f_{c} f_{b}^{2} \rho^{2}}, \\
& 0=g^{\prime \prime}-\frac{\left(g^{\prime}\right)^{2}}{g}-\frac{g^{2} P^{2}\left(K_{2}^{\prime}\right)^{2}}{9 h f_{a} f_{b}}+\frac{\left(K_{1}^{\prime}\right)^{2}}{8 h f_{b}^{2} P^{2}}+\frac{\left(K_{3}^{\prime}\right)^{2}}{8 h f_{a}^{2} P^{2}}+\left(\frac{f^{\prime}}{f}+\frac{f_{c}^{\prime}}{2 f_{c}}+\frac{f_{b}^{\prime}}{f_{b}}+\frac{f_{a}^{\prime}}{f_{a}}-\frac{3}{\rho}\right) g^{\prime} \\
& +\frac{9\left(K_{3}-K_{1}\right)^{2}}{16 f_{a} f_{b} h f f_{c} \rho^{2} P^{2}}-\frac{g^{2}\left(\left(f_{a}^{2}+f_{b}^{2}\right) K_{2}^{2}+4 f_{b}^{2}\left(1-K_{2}\right)\right) P^{2}}{2 h f f_{c} f_{a}^{2} f_{b}^{2} \rho^{2}} .
\end{aligned}
$$

Additionally we have the first order constraint

$$
\begin{aligned}
0= & \frac{\left(K_{1}^{\prime}\right)^{2}}{P^{2}}+\frac{\left(K_{3}^{\prime}\right)^{2} f_{b}^{2}}{f_{a}^{2} P^{2}}+\frac{8 g^{2} P^{2}\left(K_{2}^{\prime}\right)^{2} f_{b}}{9 f_{a}}+\frac{4 h\left(g^{\prime}\right)^{2} f_{b}^{2}}{g}-4 h g\left(f_{b}^{\prime}\right)^{2}+\frac{4 g f_{b}^{2}\left(h^{\prime}\right)^{2}}{h} \\
& -\frac{4 h g\left(f_{a}^{\prime}\right)^{2} f_{b}^{2}}{f_{a}^{2}}+\left(\frac{24 h g f_{b}^{2}}{f \rho}-\frac{4 h g f_{b}^{2} f_{c}^{\prime}}{f f_{c}}-\frac{8 h g f_{b} f_{b}^{\prime}}{f}-\frac{4 g f_{b}^{2} h^{\prime}}{f}-\frac{8 h g f_{b}^{2} f_{a}^{\prime}}{f f_{a}}\right) f^{\prime}+\frac{64 h g f_{b} f_{b}^{\prime}}{\rho} \\
& +\left(\frac{32 h g f_{b}^{2}}{f_{c} \rho}-\frac{8 h g f_{b} f_{b}^{\prime}}{f_{c}}-\frac{8 h g f_{b}^{2} f_{a}^{\prime}}{f_{c} f_{a}}\right) f_{c}^{\prime}-\frac{16 h g f_{b} f_{b}^{\prime} f_{a}^{\prime}}{f_{a}}+\frac{32 g f_{b}^{2} h^{\prime}}{\rho}+\frac{64 h g f_{b}^{2} f_{a}^{\prime}}{f_{a} \rho} \\
& -\frac{4 g^{2}\left(K_{2}^{2}\left(f_{a}^{2}+f_{b}^{2}\right)+4 f_{b}^{2}\left(1-K_{2}\right)\right) P^{2}}{f f_{c} f_{a}^{2} \rho^{2}}-\frac{g K_{2}\left(K_{3}-K_{1}\right)\left(K_{2}\left(K_{3}-K_{1}\right)+4 K_{1}\right)}{h f f_{c} f_{a}^{2} \rho^{2}}
\end{aligned}
$$




$$
\begin{aligned}
& -\frac{9 f_{b}\left(K_{3}-K_{1}\right)^{2}}{2 f_{a} f f_{c} \rho^{2} P^{2}}-\frac{4 g K_{1}^{2}}{h f f_{c} f_{a}^{2} \rho^{2}}-\frac{96 h g f_{b}^{2}}{\rho^{2}}-\frac{18 h f_{a} g f_{b}}{f f_{c} \rho^{2}}+\frac{36 h g f_{b}^{2}}{f f_{c} \rho^{2}}+\frac{96 h g f_{b}}{f \rho^{2}}-\frac{18 h g f_{b}^{3}}{f f_{c} f_{a} \rho^{2}} \\
& +\frac{96 h g f_{b}^{2}}{f f_{a} \rho^{2}}-\frac{32 h f_{c} g f_{b}}{f f_{a} \rho^{2}}+\frac{48 h^{2} g f_{b}^{2} \mu^{2}}{f}
\end{aligned}
$$

We explicitly verified that the constraint (A.45) is consistent with (A.36)-(A.44). Thus the second-order equation for $f_{c}$ (A.39) can be eliminated in favor of the constraint equation (A.45) where $f_{c}^{\prime}$ enters linearly. In total, we expect that a solution is specified by $8 \times 2+1 \times 1=17$ parameters ( 8 second-order equations for $f_{a, b}, K_{1,2,3}, h, g, f$ and a single first-order equation for $f_{c}$ ).

The general UV (as $\rho \rightarrow 0$ ) asymptotic solution of (A.36)-(A.45) describing the black hole on the warped deformed conifold with fluxes and $S^{3}$ horizon (from the effective $5 \mathrm{~d}$ perspective (3.1)), i.e., the thermal states of the cascading gauge theory on $S^{3}$, takes the form

$$
\begin{aligned}
f_{a}= & +f_{a, 1,0} \rho+\rho^{2}\left(\frac{3}{16} \mu^{2} P^{2} g_{s}+\frac{1}{4} f_{a, 1,0}^{2}\right)+\rho^{3} f_{a, 3,0}+\mathcal{O}\left(\rho^{4} \ln \rho\right) \\
f_{b}= & +f_{a, 1,0} \rho+\rho^{2}\left(\frac{3}{16} \mu^{2} P^{2} g_{s}+\frac{1}{4} f_{a, 1,0}^{2}\right)-\rho^{3} f_{a, 3,0}+\mathcal{O}\left(\rho^{4} \ln \rho\right) \\
f_{c}= & +f_{a, 1,0} \rho+\rho^{2}\left(\frac{1}{4} \mu^{2} P^{2} g_{s}+\frac{1}{4} f_{a, 1,0}^{2}\right)+\mathcal{O}\left(\rho^{4} \ln \rho\right), \\
h= & \frac{1}{8} P^{2} g_{s}+\frac{1}{4} K_{0}-\frac{1}{2} P^{2} g_{s} \ln \rho+\rho\left(-\frac{1}{2} K_{0} f_{a, 1,0}+P^{2} g_{s} f_{a, 1,0} \ln \rho\right)+\rho^{2}\left(\frac{1}{9} P^{4} g_{s}^{2} \mu^{2}\right. \\
& \left.-\frac{1}{24} K_{0} P^{2} g_{s} \mu^{2}-\frac{1}{4} P^{2} g_{s} f_{a, 1,0}^{2}+\frac{5}{8} K_{0} f_{a, 1,0}^{2}+\left(\frac{1}{12} P^{4} g_{s}^{2} \mu^{2}-\frac{5}{4} P^{2} g_{s} f_{a, 1,0}^{2}\right) \ln \rho\right) \\
& +\rho^{3}\left(-\frac{3}{8} f_{a, 1,0} P^{4} g_{s}^{2} \mu^{2}+\frac{1}{8} K_{0} f_{a, 1,0} \mu^{2} P^{2} g_{s}+\frac{11}{24} f_{a, 1,0}^{3} P^{2} g_{s}-\frac{5}{8} K_{0} f_{a, 1,0}^{3}\right. \\
& \left.+\left(-\frac{1}{4} f_{a, 1,0} P^{4} g_{s}^{2} \mu^{2}+\frac{5}{4} f_{a, 1,0}^{3} P^{2} g_{s}\right) \ln \rho\right)+\mathcal{O}\left(\rho^{4} \ln ^{2} \rho\right), \\
K_{1}= & K_{0}-2 P^{2} g_{s} \ln \rho+f_{a, 1,0} P^{2} g_{s} \rho+\rho^{2}\left(\frac{1}{4} P^{2} g_{s}\left(3 P^{2} g_{s} \mu^{2}+K_{0} \mu^{2}-f_{a, 1,0}^{2}\right)\right. \\
& \left.-\frac{1}{2} P^{4} g_{s}^{2} \mu^{2} \ln \rho\right)+\rho^{3}\left(\frac{1}{12} P^{2} g_{s}\left(f_{a, 1,0}^{3}-6 P^{2} g_{s} f_{a, 1,0} \mu^{2}-3 K_{0} f_{a, 1,0} \mu^{2}+8 f_{a, 3,0}+8 k_{2,3,0}\right)\right. \\
& \left.+\frac{1}{2} P^{2} g_{s}\left(P^{2} g_{s} f_{a, 1,0} \mu^{2}+4 f_{a, 3,0}\right) \ln \rho\right)+\mathcal{O}\left(\rho^{4} \ln ^{2} \rho\right), \\
& \left.+\frac{1}{2} P^{2} g_{s}\left(P^{2} g_{s} f_{a, 1,0} \mu^{2}-4 f_{a, 3,0}\right) \ln \rho\right)+\mathcal{O}\left(\rho^{4} \ln ^{2} \rho\right), \\
K_{2}= & +\rho^{3}\left(k_{2,3,0}+3 f_{a, 3,0} \ln \rho\right)+\mathcal{O}\left(\rho^{4} \ln \rho\right), \\
K_{3}= & K_{0}-2 P^{2} g_{s} \ln \rho+P^{2} g_{s} f_{a, 1,0} \rho+\rho^{2}\left(\frac{1}{4} P^{2} g_{s}\left(3 P^{2} g_{s} \mu^{2}+K_{0} \mu^{2}-f_{a, 1,0}^{2}\right)\right. \\
& \left.-\frac{1}{2} \mu^{2} P^{4} g_{s}^{2} \ln \rho\right)+\rho^{3}\left(\frac{1}{12} P^{2} g_{s}\left(f_{a, 1,0}^{3}-6 P^{2} f_{a, 1,0} g_{s} \mu^{2}-3 K_{0} f_{a, 1,0} \mu^{2}-8 f_{a, 3,0}-8 k_{2,3,0}\right)\right. \\
& (\mathrm{A} .51) 52) \\
&
\end{aligned}
$$




$$
\begin{aligned}
g= & g_{s}\left(1-\frac{1}{4} \mu^{2} P^{2} g_{s} \rho^{2}+\frac{1}{4} \mu^{2} f_{a, 1,0} P^{2} g_{s} \rho^{3}+\mathcal{O}\left(\rho^{4} \ln \rho\right)\right) \\
f= & 1+\rho^{2}\left(\frac{1}{8} \mu^{2} P^{2} g_{s}+\frac{1}{4} \mu^{2} K_{0}-\frac{1}{2} \mu^{2} P^{2} g_{s} \ln \rho\right)+\rho^{3}\left(\frac{1}{8} \mu^{2} f_{a, 1,0} P^{2} g_{s}-\frac{1}{4} \mu^{2} f_{a, 1,0} K_{0}\right. \\
& \left.+\frac{1}{2} \mu^{2} f_{a, 1,0} P^{2} g_{s} \ln \rho\right)+\rho^{4}\left(f_{4,0}-\left(\frac{13}{144} \mu^{4} P^{4} g_{s}^{2}+\frac{1}{12} \mu^{4} K_{0} P^{2} g_{s}+\frac{3}{8} \mu^{2} P^{2} g_{s} f_{a, 1,0}^{2}\right) \ln \rho\right. \\
& \left.+\frac{1}{12} \mu^{4} P^{4} g_{s}^{2} \ln ^{2} \rho\right)+\mathcal{O}\left(\rho^{5} \ln ^{2} \rho\right) .
\end{aligned}
$$

It is characterized by the cascading gauge theory defining parameters (A.20). Additionally, there are 9 normalizable coefficients, related to the diffeomorphism parameter $\alpha(3.17)$ and the expectation values of the various operators in the boundary theory

$$
\left\{f_{a, 1,0}, f_{a, 3,0}, k_{2,3,0}, f_{4,0}, f_{c, 4,0}, g_{4,0}, f_{a, 6,0}, k_{2,7,0}, f_{c, 8,0}\right\} .
$$

Unlike the topologically distinct background geometries representing the $\mathcal{V}_{A}$ or $\mathcal{V}_{B}$ vacua of the cascading gauge theories, the topology of the background geometry representing the deconfined thermal states ${ }^{30}$ of the cascading gauge theory $\mathcal{T}_{\text {decon }}$ is unique: here, the Euclidean time direction smoothly shrinks to zero size, with the boundary spatial $S^{3}$ and the conifold cycles remaining finite. Introducing

$$
f_{a, b, c}^{h} \equiv y^{2} f_{a, b, c}, \quad h^{h} \equiv y^{-4} h,
$$

the IR asymptotic expansion takes form

$$
\begin{array}{rlrl}
f_{a, b, c}^{h} & =\sum_{n=0} f_{a, b, c, n}^{h} y^{n}, h^{h} & =\sum_{n=0} h_{n}^{h} y^{n}, \quad K_{1,2,3}=\sum_{n=0} K_{1,2,3, n}^{h} y^{n}, \\
g & =\sum_{n=0} g_{n}^{h} y^{n}, \quad f & =\sum_{n=1} f_{n}^{h} y^{n}, &
\end{array}
$$

and is characterized by 9 parameters:

$$
\left\{f_{a, 0}^{h}, f_{b, 0}^{h}, f_{c, 0}^{h}, h_{0}^{h}, K_{1,0}^{h}, K_{2,0}^{h}, K_{3,0}^{h}, g_{0}^{h}, f_{1}^{h}\right\} .
$$

A combination of these parameters is related to the Hawking temperature $T$ of the black hole:

$$
T=\frac{f_{1}^{h}}{4 \pi \sqrt{h_{0}^{h}}} .
$$

Note that given (A.57),

$$
\begin{aligned}
& \frac{1}{h^{1 / 2} \rho^{2}}\left(-f d t^{2}\right)+\frac{h^{1 / 2}}{f \rho^{2}}(d \rho)^{2} \quad \underbrace{\longrightarrow}_{t \rightarrow i t_{E}} \frac{f}{\sqrt{h^{h}}} d t_{E}^{2}+\frac{\sqrt{h^{h}}}{f}(d y)^{2} \\
& \underbrace{\longrightarrow}_{y \equiv z^{2} \rightarrow 0} \frac{4 \sqrt{h_{0}^{h}}}{f_{1}^{h}}\left(z^{2} \frac{\left(f_{1}^{h}\right)^{2}}{4 h_{0}^{h}} d t_{E}^{2}+(d z)^{2}\right),
\end{aligned}
$$

\footnotetext{
${ }^{30}$ Dual to bulk geometries with a regular Schwarzschild horizon, see (3.19).
} 
i.e., the compactified Euclidean time direction $S^{1}$ indeed smoothly shrinks to zero size as $z \rightarrow 0$, provided $t_{E} \sim t_{E}+\frac{1}{T}$ with the temperature given by (A.59). It is important to emphasize that $\mathcal{T}_{\text {decon }}$ deconfined thermal states defined by (A.57) have either $\mathrm{U}(1)$ or $\mathbb{Z}_{2}$ chiral symmetry - the chiral symmetry is unbroken in the former $\left(\mathcal{T}_{\text {decon }}^{s}\right.$ deconfined thermal states), and is spontaneously broken in the latter $\left(\mathcal{T}_{\text {decon }}^{b}\right.$ deconfined thermal states). Specifically, unbroken chiral symmetry dictates (3.28), leading to

$$
\begin{aligned}
& \text { UV : } f_{a, 3,0}=0, \quad k_{2,3,0}=0, \quad k_{2,7,0}=0 \text {; } \\
& \text { IR : } \quad f_{b}^{h}=f_{a}^{h}, \quad K_{3,0}^{h}=K_{1,0}^{h}, \quad K_{2,0}^{h}=1 .
\end{aligned}
$$

\section{A.3 Holographic renormalization of the effective action (3.1)}

The holographic renormalization of the theory (3.1) was discussed in [25] (see also appendix A.2 of [4]). We review here the expressions necessary to recover various thermodynamic quantities of the black holes on the warped deformed conifold with fluxes, and the Casimir energies of the $S^{3}$ conifold vacua.

The SO(4) invariant five-dimensional metric ansatzes (3.15) and (3.19), convenient for the numerical computations, differ depending whether or not the bulk geometry of $\mathcal{M}_{5}$ has a horizon. To present the common expressions for the holographic renormalization we parameterize the full ten-dimensional metric (3.3) as

$$
d s_{10}^{2}=-c_{1}^{2} d t^{2}+c_{2}^{2}\left(d S^{3}\right)^{2}+c_{3}^{2}(d \rho)^{2}+\Omega_{1}^{2} g_{5}^{2}+\Omega_{2}^{2}\left(g_{3}^{2}+g_{4}^{2}\right)+\Omega_{3}^{2}\left(g_{1}^{2}+g_{2}^{2}\right),
$$

where $c_{i}=c_{i}(\rho)$ and $\Omega_{i}=\Omega_{i}(\rho)$. Note that $\Omega_{i}$ are parameterized as in (3.14), and

(A) [no horizon] : $\quad c_{1}=h^{-1 / 4} \rho^{-1}, \quad c_{2}=h^{-1 / 4} \rho^{-1} \mu^{-1} f_{1}, \quad c_{3}=h^{1 / 4} \rho^{-1}$;

(B) [horizon] : $\quad c_{1}=h^{-1 / 4} \rho^{-1} f^{1 / 2}, \quad c_{2}=h^{-1 / 4} \rho^{-1} \mu^{-1}, \quad c_{3}=h^{1 / 4} \rho^{-1} f^{-1 / 2}$.

Following [25], the renormalized five-dimensional effective action with a cutoff $\rho=\hat{\rho}$ takes form

$$
S_{5, \hat{\rho}}^{\mathrm{renom}}=S_{5, \rho \geq \hat{\rho}}^{\mathrm{bulk}}+S_{G H, \hat{\rho}}+S_{\mathrm{ct}, \hat{\rho}} .
$$

$S_{5, \rho \geq \hat{\rho}}^{\text {bulk }}$ is the regularized bulk action (3.1) with $\partial \mathcal{M}_{5}$ at $\rho=\hat{\rho}$, here ${ }^{\prime} \equiv \partial_{\rho}$,

$$
\begin{aligned}
S_{5, \rho \geq \hat{\rho}}^{\text {bulk }} & =\frac{108}{16 \pi G_{5}} \operatorname{vol}_{S^{3}} \int d t \int_{\hat{\rho}}^{+\infty} d \rho c_{1} c_{2}^{3} c_{3} \times \Omega_{1} \Omega_{2}^{2} \Omega_{3}^{2} \times\left\{R_{10}+\cdots\right\} \\
& =\frac{108}{16 \pi G_{5}} \operatorname{vol}_{S^{3}} \int d t \int_{\hat{\rho}}^{+\infty} d \rho\left[-\frac{2 c_{1}^{\prime} c_{2}^{3} \Omega_{1} \Omega_{2}^{2} \Omega_{3}^{2}}{c_{3}}\right]^{\prime} \\
& =\frac{108}{16 \pi G_{5}} \operatorname{vol}_{S^{3}} \times \int d t \times\left[\frac{2 c_{1}^{\prime} c_{2}^{3} \Omega_{1} \Omega_{2}^{2} \Omega_{3}^{2}}{c_{3}}\right]_{+\infty}^{\hat{\rho}},
\end{aligned}
$$

where in the second line we used the equations of motion (A.1)-(A.10) or (A.36)-(A.45) to represent the bulk integral as a total derivative. 
$S_{G H, \hat{\rho}}$ is the generalized Gibbons-Hawking term, evaluated at the regularization boundary $\partial \mathcal{M}_{5}$,

$$
\begin{aligned}
S_{G H, \hat{\rho}} & =\frac{108}{16 \pi G_{5}} \times 2 \int_{\partial \mathcal{M}_{5}} \operatorname{vol}_{\partial \mathcal{M}_{5}} \Omega_{1} \Omega_{2}^{2} \Omega_{3}^{2}\left(\nabla_{\mu} n^{\mu}+n^{\mu} \nabla_{\mu} \ln \left(\Omega_{1} \Omega_{2}^{2} \Omega_{3}^{2}\right)\right) \\
& =\frac{108}{16 \pi G_{5}} \operatorname{vol}_{S^{3}} \times \int d t \times\left.\frac{2}{c_{3}}\left(c_{1} c_{2}^{3} \Omega_{1} \Omega_{2}^{2} \Omega_{3}^{2}\right)^{\prime}\right|_{\rho=\hat{\rho}},
\end{aligned}
$$

where $n^{\mu}$ is a unit space-like vector orthogonal to the four-dimensional boundary $\partial \mathcal{M}_{5}$.

$S_{\mathrm{ct}, \hat{\rho}}$ is the counter-term action, ${ }^{31}$ evaluated at the regularization boundary $\partial \mathcal{M}_{5}$,

$$
S_{\mathrm{ct}, \hat{\rho}}=\frac{1}{16 \pi G_{5}} \int_{\partial \mathcal{M}_{5}} \operatorname{vol}_{\partial \mathcal{M}_{5}} \mathcal{L}^{\text {counter }},
$$

where, in the "minimal subtraction scheme" [25],

$$
\begin{aligned}
\mathcal{L}^{\text {counter }}= & \hat{K}-2 \hat{\Omega}_{1}^{4}-8 \hat{\Omega}_{2}^{4}+\mathcal{A}_{4}+\mathcal{R}_{\gamma} \hat{\Omega}_{1}^{2}\left(-\frac{1}{12} \hat{K}+\frac{1}{12} P^{2} e^{\Phi}-\frac{1}{6} \hat{\Omega}_{1}^{4}+\mathcal{B}_{2}\right) \\
& +\mathcal{R}_{\gamma}^{2} \times \mathcal{L}_{\mathcal{R}^{2}}^{0}+\mathcal{R}_{a b} \mathcal{R}_{\gamma}^{a b} \times \mathcal{L}_{\mathcal{R} i c^{2}}^{0} \\
X_{a} \equiv & \left(1-\frac{\hat{\Omega}_{2}^{2}}{\hat{\Omega}_{1}^{2}}\right), \quad \mathcal{A}_{4}=\frac{18}{5} X_{a}^{2} \hat{\Omega}_{1}^{4}, \quad \mathcal{B}_{2}=X_{a}\left(\frac{1}{6} \hat{K}-\frac{1}{30} P^{2} e^{\Phi}\right) \\
\mathcal{L}_{\mathcal{R}^{2}}^{0}= & -\frac{1}{144} P^{4} e^{2 \Phi} \ln ^{3} \rho-\frac{1}{96} P^{2} e^{\Phi} \ln ^{2} \rho \hat{K}-\frac{1}{192} \ln \rho \hat{K}^{2} \\
& +\left(\frac{1}{96}+4 \kappa_{1}\right) P^{4} e^{2 \Phi} \ln ^{2} \rho+\left(\frac{1}{96}+4 \kappa_{1}\right) P^{2} e^{\Phi} \ln \rho \hat{K} \\
& +\left(\kappa_{1}+\frac{1}{1152}\right) \hat{K}^{2}+\left(2 \kappa_{2}-\frac{43}{2304}\right) P^{4} e^{2 \Phi} \ln \rho \\
& +\left(\kappa_{2}-\frac{13}{1152}\right) P^{2} e^{\Phi} \hat{K}+\kappa_{3} P^{4} e^{2 \Phi}, \\
\mathcal{L}_{\mathcal{R} i c^{2}}^{0}= & \frac{1}{48} P^{4} e^{2 \Phi} \ln ^{3} \rho+\frac{1}{32} P^{2} e^{\Phi} \ln ^{2} \rho \hat{K}+\frac{1}{64} \ln \rho \hat{K}^{2}+\left(-\frac{1}{32}-12 \kappa_{1}\right) P^{4} e^{2 \Phi} \ln ^{2} \rho \\
& +\left(-\frac{1}{32}-12 \kappa_{1}\right) P^{2} e^{\Phi} \ln \rho \hat{K}+\left(-\frac{1}{256}-3 \kappa_{1}\right) \hat{K}^{2} \\
& +\left(\frac{43}{768}-6 \kappa_{2}\right) P^{4} e^{2 \Phi} \ln \rho+\left(\frac{5}{192}-3 \kappa_{2}\right) P^{2} e^{\Phi} \hat{K} \\
& +\left(\frac{541}{138240}-3 \kappa_{3}\right) P^{4} e^{2 \Phi}
\end{aligned}
$$

with [4]

$$
\hat{K}=\frac{1}{2}\left(K_{1}+K_{3}\right), \quad \hat{\Omega}_{1}=3 \Omega_{1}, \quad \hat{\Omega}_{2}=\frac{\sqrt{6}}{2}\left(\Omega_{2}+\Omega_{3}\right),
$$

and, for a specific choice of the five-dimensional background metric in (A.62),

$$
\mathcal{R}_{\gamma}=\frac{6}{c_{2}^{2}}, \quad \mathcal{R}_{a b \gamma} \mathcal{R}_{\gamma}^{a b}=\frac{12}{c_{2}^{4}}, \quad \square_{\gamma} \mathcal{R}_{\gamma}=0 .
$$

\footnotetext{
${ }^{31}$ This counter-term action renormalizes the effective action, the boundary stress-energy tensor and the expectation values of all the relevant and the marginal operators of the boundary theory. Additional counterterms are necessary to remove power-law divergences of the irrelevant operators of the cascading gauge theory, see [25] for more details.
} 
Notice that the holographic renormalization in the minimal subtraction ${ }^{32}$ has a 3 parameter ambiguity - these are the coefficients $\kappa_{1} \cdots \kappa_{3}$ in (A.70) and (A.71) parameterizing finite as $\rho \rightarrow 0$ counterterms

$$
\begin{aligned}
\mathcal{L}_{\text {finite }}^{\text {counter }}= & \left(\kappa_{1}\left(\hat{K}+2 P^{2} e^{\Phi} \ln \rho\right)^{2}+\kappa_{2}\left(\hat{K}+2 P^{2} e^{\Phi} \ln \rho\right) P^{2} e^{\Phi}+\kappa_{3} P^{4} e^{2 \Phi}\right) \\
& \times\left(\mathcal{R}_{\gamma}^{2}-3 \mathcal{R}_{a b} \mathcal{R}_{\gamma}^{a b}\right)
\end{aligned}
$$

The presence of the finite counterterms (A.74) is mandated [25] by the reparametrization of the radial coordinate $\rho \rightarrow \lambda \rho$ because of the explicit $\ln \rho$ dependence in (A.70) and (A.71). Indeed, it is easy to see that the reparametrization $\ln \rho \rightarrow \ln \rho+\ln \lambda$ is equivalent to

$$
\begin{array}{lll}
\kappa_{1} & \rightarrow & \kappa_{1}-\frac{1}{192} \ln \lambda ; \\
\kappa_{2} & \rightarrow & \kappa_{2}+4 \kappa_{1} \ln \lambda-\frac{1}{96} \ln ^{2} \lambda+\frac{1}{96} \ln \lambda \\
\kappa_{3} & \rightarrow & \kappa_{3}+2 \kappa_{2} \ln \lambda+4 \kappa_{1} \ln ^{2} \lambda-\frac{1}{144} \ln ^{3} \lambda+\frac{1}{96} \ln \lambda-\frac{43}{2304} \ln \lambda .
\end{array}
$$

The specific structure of the finite counter-term ambiguity, namely the combination $\left(\mathcal{R}_{\gamma}^{2}-\right.$ $3 \mathcal{R}_{a b \gamma} \mathcal{R}_{\gamma}^{a b}$ ), implies that the renormalized boundary stress-energy tensor is ambiguity free when $\partial \mathcal{M}_{5}=R \times S^{3}[10]$. This might come as a surprise, as it is well-known [26] that a finite counterterm (constant $\delta_{\mathcal{R}^{2}}$ )

$$
\delta \mathcal{L}_{\text {finite }}^{\text {counter }}=\delta_{\mathcal{R}^{2}} \times \mathcal{R}_{\gamma}^{2}
$$

would produce $\propto \delta_{\mathcal{R}^{2}} R^{2}$ ambiguity in the boundary energy, as well as contribute $\propto \delta_{\mathcal{R}^{2}} \square R$ ambiguity to the trace-anomaly. As explained in [25], a finite counterterm (A.76) is absent in the minimal subtraction since it reintroduces $\propto \delta_{\mathcal{R}^{2}} \square R$ divergences in the one-point functions of the irrelevant operators of the cascading gauge theory. If we restrict renormalization of the theory to the manifolds with $\square_{\gamma} \mathcal{R}_{\gamma}=0$, as in (A.73), such a finite counter-term is allowed.

As usual, in the present of the Schwarzschild horizon in the bulk, we can analytically continue time

$$
t \rightarrow i t_{E}, \quad t_{E} \sim t_{E}+\frac{1}{T}
$$

where $t_{E}$ is periodic with inverse temperature $T$, and identify the free energy density $\mathcal{F}$ of the black hole as

$$
\frac{1}{T} \times \frac{1}{\mu^{3}} \operatorname{vol}_{S^{3}} \times \mathcal{F}=\left.\lim _{\hat{\rho} \rightarrow 0} S_{5, \hat{\rho}}^{\mathrm{renom}}\right|_{t \rightarrow i t_{E}} .
$$

The renormalized effective action $S_{5, \hat{\rho}}^{\text {renom }}$ can further be used to compute the boundary stress-energy tensor as detailed in [25].

\footnotetext{
${ }^{32}$ Additional ambiguities arise for generic $\partial \mathcal{M}_{5}$, in particular when $\square_{\gamma} \mathcal{R}_{\gamma} \neq 0$.
} 


\section{A.4 Boundary stress-energy tensor}

In this section, using the results of sections A.1-A.3, we collect expressions for the energy density $\mathcal{E}$ and pressure $\mathcal{P}$, as well as some additional characteristics, for the vacua and the thermal states of the cascading gauge theory on $S^{3}$.

Recall that,

- $\mathcal{V}_{A}^{b}$ denotes vacua of the cascading gauge theory with spontaneously broken chiral symmetry, with topologically trivial boundary $S^{3}$ - see (A.11)-(A.19) for the UV asymptotics, and (A.23) (with (A.25)) for the IR asymptotics;

- $\mathcal{V}_{A}^{s}$ denotes vacua of the cascading gauge theory with unbroken chiral symmetry, with topologically trivial boundary $S^{3}$ - see (A.26) for the constraints on UV/IR parameters;

- $\mathcal{V}_{B}$ denotes vacua of the cascading gauge theory with spontaneously broken chiral symmetry, with topologically non-trivial boundary $S^{3}$ (the Klebanov-Strassler solution [2] is a member of this class in the boundary $S^{3}$ decompactification limit) — see (A.11)-(A.19) for the UV asymptotics, and (A.28) (with (A.30)) for the IR asymptotics;

- $\mathcal{T}_{\text {decon }}^{b}$ denotes thermal deconfined states of the cascading gauge theory with spontaneously broken chiral symmetry — see (A.46)-(A.54) for the UV asymptotics, and (A.57) (with (A.60)) for the IR asymptotics;

- $\mathcal{T}_{\text {decon }}^{s}$ denotes thermal deconfined states of the cascading gauge theory with unbroken chiral symmetry — see (A.61) for the constraints on UV/IR parameters.

\section{A.4.1 $\mathcal{V}_{A}^{b}$ vacua}

For the energy density $\mathcal{E}$ and the pressure $\mathcal{P}$ we find [25]

$$
\begin{aligned}
8 \pi G_{5} \mathcal{E}= & \left(\frac{403}{1920} P^{4} g_{s}^{2}+\frac{3}{32} K_{0} P^{2} g_{s}+\frac{1}{32} K_{0}^{2}\right) \mu^{4}+\frac{9}{32} f_{a, 1,0}^{2} \mu^{2} P^{2} g_{s}-\frac{3}{2} f_{a, 1,0} f_{a, 3,0} \\
& -3 f_{a, 4,0}+\frac{3}{2} f_{c, 4,0} \\
8 \pi G_{5} \mathcal{P}= & \left(\frac{283}{5760} P^{4} g_{s}^{2}+\frac{1}{16} K_{0} P^{2} g_{s}+\frac{1}{96} K_{0}^{2}\right) \mu^{4}-\frac{5}{32} f_{a, 1,0}^{2} \mu^{2} P^{2} g_{s}+\frac{1}{2} f_{a, 1,0} f_{a, 3,0} \\
& +f_{a, 4,0}-\frac{3}{2} f_{c, 4,0} .
\end{aligned}
$$

Additionally, the expectation value of the $\operatorname{dim}-4$ operator $\mathcal{O}_{K_{0}}$, associated with the coupling $K_{0}$, is [25]

$$
8 \pi G_{5}\left\langle\mathcal{O}_{K_{0}}\right\rangle=\left(-\frac{3}{32} K_{0}+\frac{1}{16} P^{2} g_{s}\right) \mu^{4}+\frac{3}{4} f_{a, 1,0}^{2} \mu^{2}+\frac{6\left(f_{c, 4,0}-f_{a, 4,0}\right)-3 f_{a, 1,0} f_{a, 3,0}}{P^{2} g_{s}} .
$$

As explained in details in [25], the conformal anomaly of the cascading gauge theory reads

$$
\text { conformal anomaly }=\left\langle T_{i}^{i}\right\rangle+P^{2} g_{s}\left\langle\mathcal{O}_{K_{0}}\right\rangle=-\mathcal{E}+3 \mathcal{P}+P^{2} g_{s}\left\langle\mathcal{O}_{K_{0}}\right\rangle,
$$


which vanishes here. Indeed, in any local field theory with the gravitational dual, the conformal anomaly is a linear combination of terms $I_{4}-E_{4} \propto\left(3 R_{\mu \nu} R^{\mu \nu}-R^{2}\right)$ (see (2.6) and (2.7)) and $\square R$ - both of these terms vanish for the $\mathcal{M}_{4}=R \times S^{3}$ boundary.

Note that any gravitational solution representing a $\mathcal{V}_{A}^{b}$ vacuum can be interpreted as a thermal confined state of the cascading gauge theory with spontaneously broken chiral symmetry, $\mathcal{T}_{\text {con, }}^{b}$, provided the Euclidean time direction is compactified with the inverse temperature period as in (A.77). In this case, it is easy to verify that the free energy density computed from (A.78) is exactly the same as the energy density,

$$
\mathcal{T}_{\text {con, } \mathrm{A}}^{b}: \quad \mathcal{F}=\mathcal{E}
$$

consistent with vanishing (in the supergravity or large- $N$ approximation) entropy density of the confined states.

\section{A.4.2 $\mathcal{V}_{A}^{s}$ vacua}

These vacua are the special case of the $\mathcal{V}_{A}^{b}$ vacua, subject to constraints of the unbroken chiral symmetry (A.26). Explicitly,

$$
\begin{aligned}
8 \pi G_{5} \mathcal{E} & =\left(\frac{403}{1920} P^{4} g_{s}^{2}+\frac{3}{32} K_{0} P^{2} g_{s}+\frac{1}{32} K_{0}^{2}\right) \mu^{4}-\frac{3}{32} f_{a, 1,0}^{2} \mu^{2} P^{2} g_{s}-3 f_{a, 4,0}+\frac{3}{2} f_{c, 4,0}, \\
8 \pi G_{5} \mathcal{P} & =\left(\frac{283}{5760} P^{4} g_{s}^{2}+\frac{1}{16} K_{0} P^{2} g_{s}+\frac{1}{96} K_{0}^{2}\right) \mu^{4}-\frac{1}{32} f_{a, 1,0}^{2} \mu^{2} P^{2} g_{s}+f_{a, 4,0}-\frac{3}{2} f_{c, 4,0},
\end{aligned}
$$

and

$$
8 \pi G_{5}\left\langle\mathcal{O}_{K_{0}}\right\rangle=\left(-\frac{3}{32} K_{0}+\frac{1}{16} P^{2} g_{s}\right) \mu^{4}+\frac{6\left(f_{c, 4,0}-f_{a, 4,0}\right)}{P^{2} g_{s}} .
$$

\section{A.4.3 $\mathcal{V}_{B}$ vacua}

While $\mathcal{V}_{B}$ vacua are represented by topologically district solutions in the holographic dual from those corresponding to $\mathcal{V}_{A}^{b}$ vacua, and thus have different IR asymptotics (compare (A.23) with (A.28)), both vacua have the same UV asymptotics (A.11)-(A.19). This leads to the identical expressions for the energy density $\mathcal{E}$, the pressure $\mathcal{P}$, and the expectation value of $\mathcal{O}_{K_{0}}$ operators as in (A.79)-(A.80).

Supersymmetric Klebanov-Strassler solution [2] is a decompactification $\mu \rightarrow 0$ limit of $\mathcal{V}_{B}$ vacua. Using the identifications (A.33) we compute from (A.79)-(A.80)

$$
\mathcal{E}=0, \quad \mathcal{P}=0, \quad\left\langle\mathcal{O}_{K_{0}}\right\rangle=0,
$$

as expected. 


\section{A.4.4 $\mathcal{T}_{\text {decon }}^{b}$ thermal states}

For the energy density $\mathcal{E}$ and the pressure $\mathcal{P}$ we find [25]

$$
\begin{aligned}
8 \pi G_{5} \mathcal{E}= & \left(\frac{171}{640} P^{4} g_{s}^{2}+\frac{17}{192} K_{0} P^{2} g_{s}+\frac{1}{32} K_{0}^{2}\right) \mu^{4}+\left(\frac{9}{32} K_{0}-\frac{21}{64} P^{2} g_{s}\right) f_{a, 1,0}^{2} \mu^{2}-\frac{3}{2} f_{4,0} \\
& +\frac{3}{2} f_{c, 4,0} \\
8 \pi G_{5} \mathcal{P}= & \left(-\frac{7}{5760} P^{4} g_{s}^{2}+\frac{1}{576} K_{0} P^{2} g_{s}+\frac{1}{96} K_{0}^{2}\right) \mu^{4}+\left(\frac{3}{32} K_{0}-\frac{7}{64} P^{2} g_{s}\right) f_{a, 1,0}^{2} \mu^{2} \\
& -\frac{1}{2} f_{4,0}-\frac{3}{2} f_{c, 4,0} .
\end{aligned}
$$

Additionally, the expectation value of the $\operatorname{dim}-4$ operator $\mathcal{O}_{K_{0}}$, associated with the coupling $K_{0}$, is [25]

$$
8 \pi G_{5}\left\langle\mathcal{O}_{K_{0}}\right\rangle=\left(\frac{1}{12} K_{0}+\frac{13}{48} P^{2} g_{s}\right) \mu^{4}+\frac{6 f_{c, 4,0}}{P^{2} g_{s}} .
$$

Once again, the conformal anomaly of the cascading gauge theory reads

$$
\text { conformal anomaly }=\left\langle T_{i}^{i}\right\rangle+P^{2} g_{s}\left\langle\mathcal{O}_{K_{0}}\right\rangle=-\mathcal{E}+3 \mathcal{P}+P^{2} g_{s}\left\langle\mathcal{O}_{K_{0}}\right\rangle=0,
$$

i.e., it vanishes here.

Thermal deconfined states $\mathcal{T}_{\text {decon }}^{b}$ carry the entropy density $s$,

$$
s=\frac{1}{4 G_{5}} f_{a, 0}^{h} f_{b, 0}^{h} \sqrt{f_{c, 0}^{h} h_{0}^{h}},
$$

with the equilibrium temperature $T$ given by (A.59). The basic thermodynamic relation

$$
\mathcal{F}=\mathcal{E}-s T
$$

automatically holds, with the free energy density $\mathcal{F}$ evaluated from (A.78).

The first law of the thermodynamics, i.e.,

$$
0=\frac{d \mathcal{E}}{T d s}-1
$$

is verified numerically in all solutions to an accuracy $\sim 10^{-7}$ for the Klebanov-Tseytlin black holes, and an accuracy $\sim 10^{-5}$ for the Klebanov-Strassler black holes - see figure 35 for a typical example.

\section{A.4.5 $\mathcal{T}_{\text {decon }}^{s}$ thermal states}

These thermal states are the special case of $\mathcal{T}_{\text {decon }}^{b}$ thermal states, subject to constraints of the unbroken chiral symmetry (A.61). We have identical expressions to (A.86) and (A.87) for the energy density $\mathcal{E}$, the pressure $\mathcal{P}$, and the expectation value of $\mathcal{O}_{K_{0}}$ because they do not depend on $\left\{f_{a, 3,0}, k_{2,3,0}, k_{2,7,0}\right\}$.

The equilibrium temperature $T$ is still given by (A.59), while for the entropy density we have

$$
s=\frac{1}{4 G_{5}}\left(f_{a, 0}^{h}\right)^{2} \sqrt{f_{c, 0}^{h} h_{0}^{h}} .
$$




\section{B Fluctuations about $\mathcal{V}_{A}^{s}$ vacua}

Introducing

$$
f_{a} \equiv f_{3}+\delta f, f_{b} \equiv f_{3}-\delta f, K_{1} \equiv K+\delta k_{1}, K_{3} \equiv K-\delta k_{1}, K_{2} \equiv 1+\delta k_{2},
$$

we obtain from (A.1)-(A.10) decoupled linearized equations for fluctuations $\delta f(\rho), \delta k_{1}(\rho)$, $\delta k_{2}(\rho)$ about $\mathcal{V}_{A}^{s}$ vacua (see section 5.2):

$$
\begin{aligned}
0= & \delta f^{\prime \prime}+\left(\frac{3 f_{1}^{\prime}}{f_{1}}+\frac{f_{c}^{\prime}}{2 f_{c}}-\frac{3}{\rho}\right) \delta f^{\prime}-\frac{K^{\prime}}{2 f_{3} h P^{2} g} \delta k_{1}^{\prime}+\left(\frac{3 h \mu^{2}}{2 f_{1}^{2}}+\frac{\left(h^{\prime}\right)^{2}}{8 h^{2}}-\frac{3 h^{\prime} f_{1}^{\prime}}{4 h f_{1}}+\frac{\left(g^{\prime}\right)^{2}}{8 g^{2}}\right. \\
& -\frac{3\left(f_{1}^{\prime}\right)^{2}}{2 f_{1}^{2}}-\frac{3 f_{1}^{\prime} f_{3}^{\prime}}{f_{1} f_{3}}-\frac{3 f_{1}^{\prime} f_{c}^{\prime}}{4 f_{1} f_{c}}+\frac{\left(f_{3}^{\prime}\right)^{2}}{4 f_{3}^{2}}-\frac{f_{3}^{\prime} f_{c}^{\prime}}{2 f_{c} f_{3}}+\frac{h^{\prime}}{h \rho}-\frac{3 f_{1}^{\prime}}{2 f_{1} \rho}+\frac{5}{\rho^{2}}-\frac{9}{f_{c} \rho^{2}}+\frac{6}{f_{3} \rho^{2}}+\frac{3 f_{c}}{\rho^{2} f_{3}^{2}} \\
& \left.-\frac{K^{2}}{8 h^{2} f_{c} \rho^{2} f_{3}^{4}}-\frac{7\left(K^{\prime}\right)^{2}}{16 h f_{3}^{2} P^{2} g}-\frac{9 P^{2} g}{4 h f_{3}^{2} f_{c} \rho^{2}}\right) \delta f-\frac{2 P^{2} g}{f_{3} h f_{c} \rho^{2}} \delta k_{2}, \\
0= & \delta k_{1}^{\prime \prime}+\left(\frac{f_{c}^{\prime}}{2 f_{c}}-\frac{h^{\prime}}{h}+\frac{3 f_{1}^{\prime}}{f_{1}}-\frac{g^{\prime}}{g}-\frac{3}{\rho}\right) \delta k_{1}^{\prime}+\frac{2 K^{\prime}}{f_{3}} \delta f^{\prime}+\left(\frac{4 K P^{2} g}{h f_{3}^{3} f_{c} \rho^{2}}-\frac{2 K^{\prime} f_{3}^{\prime}}{f_{3}^{2}}\right) \delta f \\
& +\frac{2 K P^{2} g}{h \rho^{2} f_{c} f_{3}^{2}} \delta k_{2}-\frac{9}{f_{c} \rho^{2}} \delta k_{1}, \\
0= & \delta k_{2}^{\prime \prime}+\left(\frac{3 f_{1}^{\prime}}{f_{1}}-\frac{h^{\prime}}{h}+\frac{f_{c}^{\prime}}{2 f_{c}}+\frac{g^{\prime}}{g}-\frac{3}{\rho}\right) \delta k_{2}^{\prime}+\frac{9 K}{2 h f_{3}^{2} f_{c} P^{2} g \rho^{2}} \delta k_{1}-\frac{9}{f_{c} \rho^{2}} \delta k_{2}-\frac{18}{f_{3} f_{c} \rho^{2}} \delta f .
\end{aligned}
$$

We turn on a finite source term ${ }^{33} \mathrm{~m}$, (a non-normalizable coefficient for $\delta f$ ) explicitly breaking the chiral symmetry $\mathrm{U}(1) \rightarrow \mathbb{Z}_{2}$, leading to

$$
\begin{aligned}
\delta f= & m \rho+\frac{1}{2} f_{a, 1,0} m \rho^{2}+\rho^{3}\left(\delta f_{3,0}+\frac{1}{8} m \mu^{2}\left(K_{0}-10 P^{2} g_{s}\right) \ln \rho-\frac{1}{8} m \mu^{2} P^{2} g_{s} \ln ^{2} \rho\right) \\
& +\mathcal{O}\left(\rho^{4} \ln ^{2} \rho\right), \\
\delta k_{1}= & -\frac{1}{2} P^{2} g_{s} m \rho+\frac{1}{4} P^{2} g_{s} m f_{a, 1,0} \rho^{2}+\rho^{3}\left(-\frac{1}{1728} P^{2} g_{s}\left(131 P^{2} g_{s} m \mu^{2}+330 K_{0} m \mu^{2}\right.\right. \\
& \left.-432 f_{a, 1,0}^{2} m-1152 \delta f_{3,0}-1152 \delta k_{2,3,0}\right)-\frac{1}{288} P^{2} g_{s}\left(263 P^{2} g_{s} m \mu^{2}+168 K_{0} m \mu^{2}\right. \\
& \left.\left.-576 \delta f_{3,0}\right) \ln \rho+\frac{1}{24} P^{2} g_{s} m \mu^{2}\left(-16 P^{2} g_{s}+3 K_{0}\right) \ln ^{2} \rho-\frac{1}{12} P^{4} g_{s}^{2} m \mu^{2} \ln ^{3} \rho\right) \\
& +\mathcal{O}\left(\rho^{4} \ln ^{3} \rho\right), \\
\delta k_{2}= & -\frac{9}{4} m \rho+\frac{9}{8} f_{a, 1,0} m \rho^{2}+\rho^{3}\left(\delta k_{2,3,0}+\left(-\frac{133}{192} m \mu^{2} P^{2} g_{s}-m \mu^{2} K_{0}+3 \delta f_{3,0}\right) \ln \rho\right. \\
& \left.+\left(-\frac{7}{8} m \mu^{2} P^{2} g_{s}+\frac{3}{16} m \mu^{2} K_{0}\right) \ln ^{2} \rho-\frac{1}{8} m \mu^{2} P^{2} g_{s} \ln ^{3} \rho\right)+\mathcal{O}\left(\rho^{4} \ln ^{3} \rho\right),
\end{aligned}
$$

as $\rho \rightarrow 0$, and (compare with the definition of $f_{a}^{h}$ in (A.22))

$$
\delta f^{h} \equiv y^{2} \delta f=\sum_{n=0} \delta f_{n}^{h} y^{2 n}, \quad \delta k_{1}=\sum_{n=0} \delta k_{1, n}^{h} y^{2 n}, \quad \delta k_{2}=\sum_{n=0} \delta k_{2, n}^{h} y^{2 n},
$$

\footnotetext{
${ }^{33}$ This is one of the two possible source terms. It is possible to turn on an independent source term for $\delta k_{1}$; such an additional source term produces conceptually identical results.
} 
as $y \equiv \frac{1}{\rho} \rightarrow 0$. It is characterized by 3 normalizable coefficients in the UV, and 3 normalizable coefficients in the IR:

$$
\begin{aligned}
\mathrm{UV}: & \left\{\delta f_{3,0}, \delta k_{2,3,0}, \delta k_{2,7,0}\right\} ; \\
\mathrm{IR}: & \left\{\delta f_{0}^{h}, \delta k_{1,0}^{h}, \delta k_{2,0}^{h}\right\},
\end{aligned}
$$

precisely as needed to identify a solution of a system of three second-order ODEs (B.2)(B.4).

\section{Fluctuations about $\mathcal{T}_{\text {decon }}^{s}$ thermal states}

Introducing

$$
f_{a} \equiv f_{3}+\delta f, f_{b} \equiv f_{3}-\delta f, K_{1} \equiv K+\delta k_{1}, K_{3} \equiv K-\delta k_{1}, K_{2} \equiv 1+\delta k_{2},
$$

we obtain from (A.36)-(A.45) decoupled linearized equations for fluctuations $\delta f(\rho), \delta k_{1}(\rho)$, $\delta k_{2}(\rho)$ about $\mathcal{T}_{\text {decon }}^{s}$ states (see section 6.3.1):

$$
\begin{aligned}
0= & \delta f^{\prime \prime}+\left(\frac{f_{c}^{\prime}}{2 f_{c}}+\frac{f^{\prime}}{f}-\frac{3}{\rho}\right) \delta f^{\prime}-\frac{K^{\prime}}{2 f_{3} h P^{2} g} \delta k_{1}^{\prime}+\left(\frac{\left(h^{\prime}\right)^{2}}{8 h^{2}}-\frac{h^{\prime} f^{\prime}}{8 h f}+\frac{\left(g^{\prime}\right)^{2}}{8 g^{2}}+\frac{\left(f_{3}^{\prime}\right)^{2}}{4 f_{3}^{2}}\right. \\
& -\frac{f_{3}^{\prime} f_{c}^{\prime}}{2 f_{3} f_{c}}-\frac{f^{\prime} f_{3}^{\prime}}{2 f_{3} f}-\frac{f^{\prime} f_{c}^{\prime}}{8 f f_{c}}+\frac{h^{\prime}}{h \rho}-\frac{5 f^{\prime}}{4 \rho f}+\frac{5}{\rho^{2}}-\frac{7\left(K^{\prime}\right)^{2}}{16 h f_{3}^{2} P^{2} g}-\frac{9 P^{2} g}{4 h f_{3}^{2} \rho^{2} f f_{c}}-\frac{9}{\rho^{2} f f_{c}} \\
& \left.+\frac{6}{f_{3} \rho^{2} f}+\frac{3 f_{c}}{f_{3}^{2} \rho^{2} f}-\frac{K^{2}}{8 h^{2} f_{3}^{4} \rho^{2} f f_{c}}-\frac{5 h \mu^{2}}{2 f}\right) \delta f-\frac{2 P^{2} g}{f_{3} h \rho^{2} f f_{c}} \delta k_{2} \\
0= & \delta k_{1}^{\prime \prime}+\left(\frac{f^{\prime}}{f}+\frac{f_{c}^{\prime}}{2 f_{c}}-\frac{h^{\prime}}{h}-\frac{g^{\prime}}{g}-\frac{3}{\rho}\right) \delta k_{1}^{\prime}+\frac{2 K^{\prime}}{f_{3}} \delta f^{\prime}+\left(\frac{4 K P^{2} g}{h f_{3}^{3} f_{c} \rho^{2} f}-\frac{2 K^{\prime} f_{3}^{\prime}}{f_{3}^{2}}\right) \delta f \\
& +\frac{2 K P^{2} g}{f_{3}^{2} h f_{c} \rho^{2} f} \delta k_{2}-\frac{9}{f f_{c} \rho^{2}} \delta k_{1}, \\
0= & \delta k_{2}^{\prime \prime}+\left(\frac{g^{\prime}}{g}+\frac{f_{c}^{\prime}}{2 f_{c}}-\frac{h^{\prime}}{h}+\frac{f^{\prime}}{f}-\frac{3}{\rho}\right) \delta k_{2}^{\prime}+\frac{9 K}{2 h f f_{c} f_{3}^{2} P^{2} g \rho^{2}} \delta k_{1}-\frac{9}{f f_{c} \rho^{2}} \delta k_{2} \\
& -\frac{18}{f_{3} f f_{c} \rho^{2}} \delta f .
\end{aligned}
$$

We find

$$
\begin{aligned}
\delta f & =\delta f_{3,0} \rho^{3}+\mathcal{O}\left(\rho^{4}\right) \\
\delta k_{1} & =\left(\frac{2}{3} P^{2} g_{s}\left(\delta f_{3,0}+\delta k_{2,3,0}\right)+2 P^{2} g_{s} \delta f_{3,0} \ln \rho\right) \rho^{3}+\mathcal{O}\left(\rho^{4} \ln \rho\right), \\
\delta k_{2} & =\left(\delta k_{2,3,0}+3 \delta f_{3,0} \ln \rho\right) \rho^{3}+\mathcal{O}\left(\rho^{4} \ln \rho\right)
\end{aligned}
$$

as $\rho \rightarrow 0$, and (compare with the definition of $f_{a}^{h}$ in (A.56))

$$
\delta f^{h} \equiv y^{2} \delta f=\sum_{n=0} \delta f_{n}^{h} y^{2 n}, \quad \delta k_{1}=\sum_{n=0} \delta k_{1, n}^{h} y^{2 n}, \quad \delta k_{2}=\sum_{n=0} \delta k_{2, n}^{h} y^{2 n} .
$$


as $y \equiv \frac{1}{\rho} \rightarrow 0$. It is characterized by 3 normalizable coefficients in the UV, and 3 normalizable coefficients in the IR:

$$
\begin{aligned}
\mathrm{UV}: & \left\{\delta f_{3,0}, \delta k_{2,3,0}, \delta k_{2,7,0}\right\} ; \\
\mathrm{IR}: & \left\{\delta f_{0}^{h}, \delta k_{1,0}^{h}, \delta k_{2,0}^{h}\right\} .
\end{aligned}
$$

Because the fluctuations describing the spontaneous chiral symmetry breaking are linearized, their overall amplitude is not fixed:

$$
\left\{\delta f, \delta k_{1}, \delta k_{2}\right\} \sim \lambda\left\{\delta f, \delta k_{1}, \delta k_{2}\right\}, \quad \lambda \equiv \text { const }
$$

We find it convenient to fix the amplitude setting $\delta f_{0}^{h}=1$. Note that we do not have this rescaling freedom when the chiral symmetry is broken explicitly, as in appendix B: such a rescaling would affect the source term $m \rightarrow \lambda m$.

\section{Conifold black holes at $\{T, \mu\} \gg \Lambda$}

Using (6.51), a new radial coordinate $x$ and parameter $q$ (6.52), we find from (A.36)-(A.45) at $n=1$ :

$$
\begin{aligned}
& 0=f_{1}^{\prime \prime}+\frac{4 q x^{3}+q x^{2}-10 x^{2}-10 x-3}{x(x+1) \Delta_{1}} f_{1}^{\prime}-\frac{x\left(q x^{2}-2 q x+2 x^{2}-q\right)}{(x+1) \Delta_{1}} f_{c, 1}^{\prime} \\
& -\frac{4 x\left(q x^{2}-2 q x+2 x^{2}-q\right)}{(x+1) \Delta_{1}} f_{3,1}^{\prime}-\frac{4 q\left(f_{1}-h_{1}\right)}{\Delta_{1}}, \\
& 0=f_{c, 1}^{\prime \prime}-\frac{5}{4 x(x+1)} f_{1}^{\prime}+\frac{\left.9 q x^{4}-2 q x^{3}+18 x^{4}-5 q x^{2}-40 x^{2}-40 x-12\right)}{4 x(x+1) \Delta_{1}} f_{c, 1}^{\prime} \\
& +\frac{x\left(q x^{2}-2 q x+2 x^{2}-q\right)}{(x+1) \Delta_{1}} f_{3,1}^{\prime}+\frac{q x^{4}+6 q x^{3}+2 x^{4}+3 q x^{2}+16 x^{3}+24 x^{2}+16 x+4}{4 x(x+1) \Delta_{1}} h_{1}^{\prime} \\
& -\frac{3}{4}\left(k_{1,1}^{\prime}\right)^{2}-\frac{4(x+1)^{2}}{\Delta_{1} x^{2}} k_{1,1}+\frac{5\left(q x^{2}+2 x^{2}+4 x+2\right)}{2 \Delta_{1} x^{2}} f_{1}-\frac{7(x+1)^{2}}{\Delta_{1} x^{2}} f_{c, 1} \\
& +\frac{20(x+1)^{2}}{\Delta_{1} x^{2}} f_{3,1}-\frac{5 q x^{2}-8 x^{2}-16 x-8}{2 \Delta_{1} x^{2}} h_{1}+\frac{3(x+1)^{2}}{\Delta_{1} x^{2}}, \\
& 0=f_{3,1}^{\prime \prime}-\frac{5}{4 x(x+1)} f_{1}^{\prime}+\frac{x\left(q x^{2}-2 q x+2 x^{2}-q\right)}{4 \Delta_{1}(x+1)} f_{c, 1}^{\prime}+\frac{1}{4}\left(k_{1,1}^{\prime}\right)^{2}-\frac{4(x+1)^{2}}{\Delta_{1} x^{2}} k_{1,1} \\
& +\frac{3 q x^{4}-2 q x^{3}+6 x^{4}-2 q x^{2}-10 x^{2}-10 x-3}{x(x+1) \Delta_{1}} f_{3,1}^{\prime}+\frac{8(x+1)^{2}}{\Delta_{1} x^{2}} f_{3,1}-\frac{(x+1)^{2}}{\Delta_{1} x^{2}} \\
& -\frac{5 q x^{2}-8 x^{2}-16 x-8}{2 \Delta_{1} x^{2}} h_{1}+\frac{q x^{4}+6 q x^{3}+2 x^{4}+3 q x^{2}+16 x^{3}+24 x^{2}+16 x+4}{4 x \Delta_{1}(x+1)} h_{1}^{\prime} \\
& +\frac{5\left(q x^{2}+2 x^{2}+4 x+2\right)}{2 \Delta_{1} x^{2}} f_{1}+\frac{5(x+1)^{2}}{\Delta_{1} x^{2}} f_{c, 1}, \\
& 0=h_{1}^{\prime \prime}+\frac{13}{4 x(x+1)} f_{1}^{\prime}-\frac{q x^{4}-10 q x^{3}+2 x^{4}-5 q x^{2}-16 x^{3}-24 x^{2}-16 x-4}{4 x(x+1) \Delta_{1}} f_{c, 1}^{\prime} \\
& -\frac{q x^{4}-10 q x^{3}+2 x^{4}-5 q x^{2}-16 x^{3}-24 x^{2}-16 x-4}{x(x+1) \Delta_{1}} f_{3,1}^{\prime}+\frac{3}{4}\left(k_{1,1}^{\prime}\right)^{2}+\frac{36(x+1)^{2}}{\Delta_{1} x^{2}} k_{1,1}
\end{aligned}
$$




$$
\begin{aligned}
& +\frac{7 q x^{4}-6 q x^{3}+14 x^{4}-7 q x^{2}-16 x^{3}-64 x^{2}-56 x-16}{4 x(x+1) \Delta_{1}} h_{1}^{\prime}-\frac{17(x+1)^{2}}{\Delta_{1} x^{2}} f_{c, 1} \\
& -\frac{13\left(q x^{2}+2 x^{2}+4 x+2\right)}{2 \Delta_{1} x^{2}} f_{1}+\frac{13 q x^{2}-72 x^{2}-144 x-72}{2 \Delta_{1} x^{2}} h_{1}-\frac{68(x+1)^{2}}{\Delta_{1} x^{2}} f_{3,1} \\
& +\frac{5(x+1)^{2}}{\Delta_{1} x^{2}}, \\
0= & k_{1,1}^{\prime \prime}+\frac{\left(2 q x^{4}+4 x^{4}-q x^{2}-10 x^{2}-10 x-3\right)}{x(x+1) \Delta_{1}} k_{1,1}^{\prime}-\frac{8(x+1)^{2}}{\Delta_{1} x^{2}}, \\
0= & g_{1}^{\prime \prime}+\frac{2 q x^{4}+4 x^{4}-q x^{2}-10 x^{2}-10 x-3}{x(x+1) \Delta_{1}} g_{1}^{\prime}-\frac{4(x+1)^{2}}{\Delta_{1} x^{2}}+\left(k_{1,1}^{\prime}\right)^{2}, \\
0= & f_{c, 1}^{\prime}+4 f_{3,1}^{\prime}+h_{1}^{\prime}+\frac{3}{\Delta_{2}}\left(q x^{2}+2 x^{2}+2 x+1\right)(2 x+1) f_{1}^{\prime}+\frac{x}{\Delta_{2}}(x+1)(2 x+1) \\
& \times\left(q x^{2}+2 x^{2}+2 x+1\right)\left(k_{1,1}^{\prime}\right)^{2}-\frac{16(x+1)^{3}}{x \Delta_{2}} k_{1,1}-\frac{6(x+1)\left(q x^{2}+2 x^{2}+4 x+2\right)}{x \Delta_{2}} f_{1} \\
& +\frac{4(x+1)^{3}}{x \Delta_{2}} f_{c, 1}+\frac{16(x+1)^{3}}{x \Delta_{2}} f_{3,1}+\frac{2(x+1)\left(3 q x^{2}+8 x^{2}+16 x+8\right)}{x \Delta_{2}} h_{1}-\frac{4(x+1)^{3}}{x \Delta_{2}},
\end{aligned}
$$

where

$$
\begin{aligned}
& \Delta_{1}=(2 x+1)\left((q+2) x^{2}+2 x+1\right), \\
& \Delta_{2}=x^{2}\left(x^{2}+6 x+3\right) q+2 x^{4}+32 x^{3}+48 x^{2}+32 x+8 .
\end{aligned}
$$

Notice that the equation for $k_{1,1}$ decouples and can be solved analytically:

$$
k_{1,1}=\frac{1}{q+1}\left(q\left(\ln \left((2+q) x^{2}+2 x+1\right)-2 \ln x\right)-2 \ln x+2 \ln (x+1)\right),
$$

where we fixed, without the loss of generality, the additive integration constant so that ${ }^{34}$

$$
k_{1,1}=\text { const }-2 \ln x+\mathcal{O}(x), \quad \text { const }=0 .
$$

The remaining equations must be solved numerically. We use the first order equation (D.7) to eliminate (algebraically) $f_{c, 1}^{\prime}$ from (D.1), (D.3)-(D.5), keep (D.6), and drop the redundant equation (D.2). Thus we end up with 4 second-order equations and a single first-order equation for $\left\{f_{1}, f_{3,1}, h_{1}, g_{1}, f_{c, 1}\right\}$.

\footnotetext{
${ }^{34}$ Choosing const $\neq 0$ simply modifies $\hat{K}_{0} \rightarrow \hat{K}_{0}+P^{2} g_{s} \times$ const.
} 
In the UV, i.e., as $x \rightarrow 0$, we have

$$
\begin{aligned}
f_{1}= & x^{2}\left(\frac{1}{2} q-2 q \ln x\right)+x^{3}\left(\left(1-\hat{f}_{c, 1,0}\right) q+4 q \ln x\right)+x^{4}\left(\hat{f}_{1,4,0}+\left(\frac{2}{3} q^{2}-6 q\right) \ln x\right) \\
& +\mathcal{O}\left(x^{5} \ln x\right), \\
f_{3,1}= & x \hat{f}_{c, 1,0}+\left(\frac{3}{4} q-\hat{f}_{c, 1,0}\right) x^{2}+\left(\hat{f}_{c, 1,0}-\frac{3}{2} q\right) x^{3}+\left(\frac{9}{4} q-\hat{f}_{c, 1,0}-\frac{2}{9} q^{2}\right) x^{4}+\mathcal{O}\left(x^{5}\right), \\
h_{1}= & \frac{1}{2}-2 \ln x+\left(2-2 \hat{f}_{c, 1,0}\right) x+\left(2 \hat{f}_{c, 1,0}-1-\frac{2}{3} q\right) x^{2}+\left(\frac{2}{3}+\frac{4}{3} q-2 \hat{f}_{c, 1,0}\right) x^{3} \\
& +\left(-\frac{17}{24}+2 \hat{f}_{c, 1,0}+\frac{11}{72} q^{2}-\frac{55}{24} q\right) x^{4}+\mathcal{O}\left(x^{5}\right), \\
g_{1}= & -q x^{2}+2 q x^{3}+x^{4}\left(\hat{g}_{1,4,0}+\left(1-q^{2}-q\right) \ln x\right)+\mathcal{O}\left(x^{5} \ln x\right), \\
f_{c, 1}= & x \hat{f}_{c, 1,0}+\left(q-\hat{f}_{c, 1,0}\right) x^{2}+\left(\hat{f}_{c, 1,0}-2 q\right) x^{3}+\left(\frac{1}{3}-\hat{f}_{c, 1,0}-\frac{5}{9} q^{2}+\frac{8}{3} q\right) x^{4} \\
& +\mathcal{O}\left(x^{5}\right),
\end{aligned}
$$

where the additive integration constant in $g_{1}$ is set to zero - it can be absorbed in $\mathcal{O}\left(P^{2} / \hat{K}_{0}\right)$ redefinition of $g_{s}$.

In the IR, i.e., as $y \equiv \frac{1}{x} \rightarrow 0$, we have

$$
\begin{array}{ll}
f_{1}=\hat{f}_{1,0}^{h}+\mathcal{O}(y), & f_{3,1}=\hat{f}_{3,1,0}^{h}+\mathcal{O}(y), \quad h_{1}=\hat{h}_{1,0}^{h}+\mathcal{O}(y), \\
g_{1}=\hat{g}_{1,0}^{h}+\mathcal{O}(y), \quad f_{c, 1}=\hat{f}_{c, 1,0}^{h}+\mathcal{O}(y) .
\end{array}
$$

Besides $q$, the solution for $\left\{f_{1}, f_{3,1}, h_{1}, g_{1}, f_{c, 1}\right\}$ is characterized by 10 parameters:

$$
\left\{\hat{f}_{c, 1,0}, \hat{f}_{1,4,0}, \hat{f}_{c, 6,0}, \hat{f}_{c, 8,0}, \hat{g}_{1,4,0}, \hat{f}_{1,0}^{h}, \hat{f}_{3,1,0}^{h}, \hat{h}_{1,0}^{h}, \hat{g}_{1,0}^{h}, \hat{f}_{c, 1,0}^{h}\right\} .
$$

One combination of these parameters determines the temperature at order $\mathcal{O}\left(P^{2} g_{s} / \hat{K}_{0}\right)$, thus, we have precisely $2 \times 4+1=9$ parameters left, necessary to specify a unique physical solution.

We set up numerics varying $q$, resulting in all parameters in (D.17), except for $\hat{f}_{1,4,0}$, being the functions of $q$. A choice of $\hat{f}_{1,4,0}$ is equivalent to fixing the overall mass scale at order $\mathcal{O}\left(P^{2} g_{s}\right)$, and does not affect results expressed as dimensionless quantities. Our specific choice of $\hat{f}_{1,4,0}$ is explained in section 6.4 .

\section{E Numerical tests}

The work presented in this paper is numerical. It is imperative that we do as many tests as possible to confirm the reliability of the results. In the rest of this section we highlight a small subset of the obvious, and less obvious (accidental) tests that we performed.

For the numerical integration - solving the boundary value problem for a system of coupled ODEs - we use the "shooting" procedure developed in [3]. Numerical integration uses a finite-difference method, thus, concerns that there could be a singularity in the solution inside the integration range is less of the concern, as it would have been for the spectral methods. Still, we would often plot the resulting functions over the integration range to inspect that the solutions are indeed free from the singularities. 

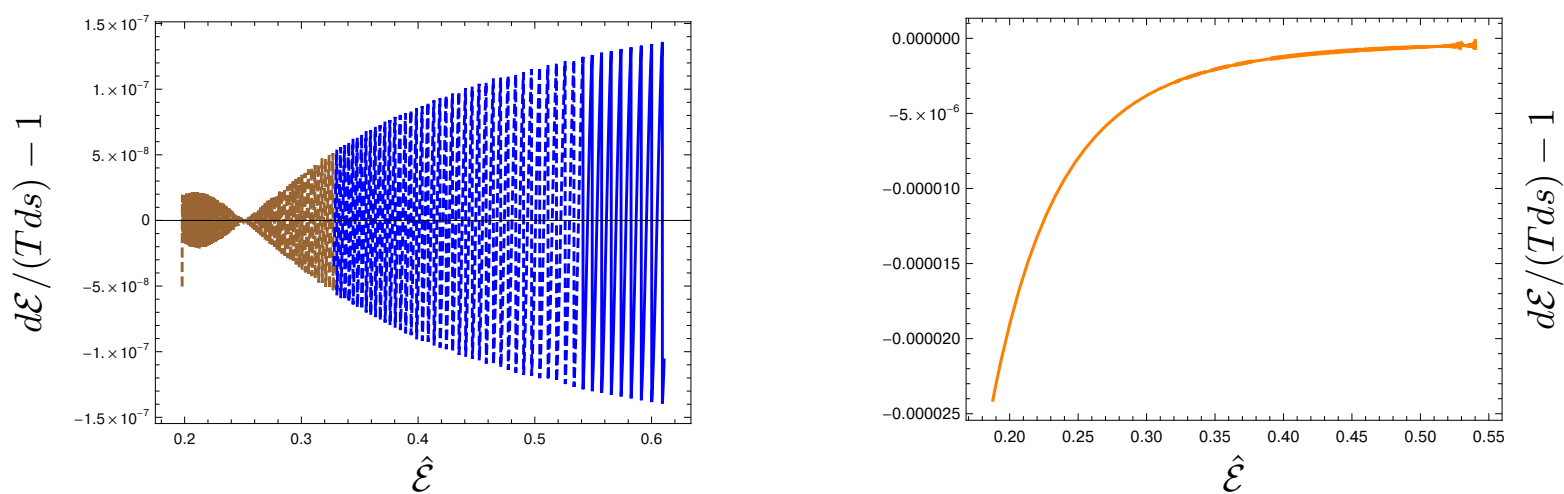

Figure 35. The first law of thermodynamics for the Klebanov-Tseytlin (the left panel) and the Klebanov-Strassler (the right panel) black holes at $\mu=\mu_{1}$, see (6.18). The color coding as in figure 30 .

\section{E.1 The first law of thermodynamics of $\mathcal{T}_{\text {decon }}^{s}$ and $\mathcal{T}_{\text {decon }}^{b}$ states at $\mu=\mu_{1}$}

In order to study the phase diagram of the black holes on the conifold we have to generate a large number of thermal spectra of these black holes. These spectra at taken at fixed $\mu / \Lambda$, with $\mu$ begin the compactification scale of the $S^{3}$ and $\Lambda$ the strong coupling scale of the cascading gauge theory. We use the holographic renormalization of appendix A.3 to compute the energy and free energy densities $\mathcal{E}$ and $\mathcal{F}$, the entropy density $s$ and the temperature $T$. When both $\mu$ and $\Lambda$ kept first, the first law of thermodynamics (A.91) must be satisfied. Note that (A.91) is a differential constraint on our numerical data we use the default tools of Wolfram Mathematica ${ }^{\circ}$ to construct interpolating functions for the collected data sets and verify that the first law of thermodynamics is satisfied for the obtained interpolation functions (which can be easily differentiated). In figure 35 we present the verification of the first law for the Klebanov-Tseytlin black holes (the left panel) and the Klebanov-Strassler black holes at select value ${ }^{35} \mu=\mu_{1}$, see (6.18). It is important to keep in mind that not only the first law validates the numerics, but it also confirmed that we correctly collected the black hole spectra using the defining parameters of the theory fixed $(\mu, \Lambda)$, and that we correctly implemented the holographic renormalization.

Results reported in figure 35 are typical:

- Klebanov-Tseytlin black holes are more symmetric than the Klebanov-Strassler black holes - the former ones require solving a coupled nonlinear system of 6 ODEs, while the latter ones has 9 ODEs. The asymptotics of the KS black holes are more involved, and contain a gravitational mode dual to a dimension-7 operator (parameter $k_{2,7,0}$ in (A.21)), which is absent in KT black holes. It is thus not a surprise that numerical accuracy of KS black holes lags behind that of KT black holes.

- Numerics of black holes on the conifold, studied here, is more challenging than that of the of the black branes discussed in $[3,4]$. The reason is apparent from the UV asymptotic expansions (A.46)-(A.54). For concreteness, focus on the expansion for $f$

\footnotetext{
${ }^{35}$ We performed this check for every single spectrum we collected.
} 
in (A.54): when $\mu \neq 0$, there are additional terms, say $\propto \mu^{4} \rho^{4} \ln ^{2} \rho$, that are present for black holes, and are absent for the black branes - these additional logarithms produce extra numerical difficulties.

- The quality of the numerics deteriorates as the simulated system is pushed into more extreme regime - the curvature of the background geometry increases, or we approach the state existence boundary (as for the Klebanov-Strassler black holes as $\mu \rightarrow \mu_{K S}$ of (6.17)), or some of the parameters specifying the solution diverge (see the left panel of figure 32$)$.

- Typically as in figure 35 (more pronounced in the left panel), the error has a highfrequency noise - this is a reflection of the discreteness in data collection, which was used to produce interpolating functions.

We stop trusting numerics when the first law constraint (A.91) violation exceeds $\sim$ $10^{-5}-10^{-4}$. Mistakes in analytical results for the holographic renormalization, or failures to keep mass scales defining the theory fixed, lead to violations of (A.91) of order $\mathcal{O}(1)$.

\section{E.2 Thermodynamics of $\mathcal{T}_{\text {decon }}^{s}$ states at $\mu=\mu_{2}$}

The parameter state of the black holes on the conifold is 3-dimensional; we have:

$\mu$ - the compactification scale of the boundary $S^{3}$;

$\Lambda$ — the strong coupling scale of the theory;

$T$ - the Hawking temperature of the black holes.

These scales have a nonlinear dependence on the numerical parameters defining the solution (A.20). For example, from (2.48),

$$
\frac{\mu^{2}}{\Lambda^{2}}=2^{-1 / 2} \mu^{2} P^{2} g_{s} e^{\frac{K_{0}}{P^{2} g_{s}}}
$$

The symmetries of the equations of motion describing the black holes, i.e., (3.22), (3.24) and (3.25), allows for an inequivalent numerical schemes. One such scheme is to set $\mu=1$, $g_{s}=1$ and $K_{0}=1$, parameterizing

$$
\frac{\mu^{2}}{\Lambda^{2}}=2^{-1 / 2} b e^{\frac{1}{b}}
$$

with $b \equiv P^{2}$, see (5.30). Note that (E.2) is a decreasing function of $b$ as $b \in[0,1]$, and then it increases again for $b>1$, retracing the same set of $\mu / \Lambda$ with $b \rightarrow \infty$, albeit with seemingly different set of defining parameters. Of course, any black hole physical observable, say $\hat{s}(\hat{\mathcal{E}})$, in reality is represented as a parametric dependence

$$
\{\hat{\mathcal{E}}(b), \hat{s}(b)\}
$$

with $\mathcal{E}$ (A.86) and $s$ (A.89) depending on $b$ implicitly via parameters (A.55) and (A.58), must 'fold on itself' for $b>1$. This is precisely what we observe in this work, and what was observed for black branes in $[3,4]$. We call this 'an obvious' numerical test. 

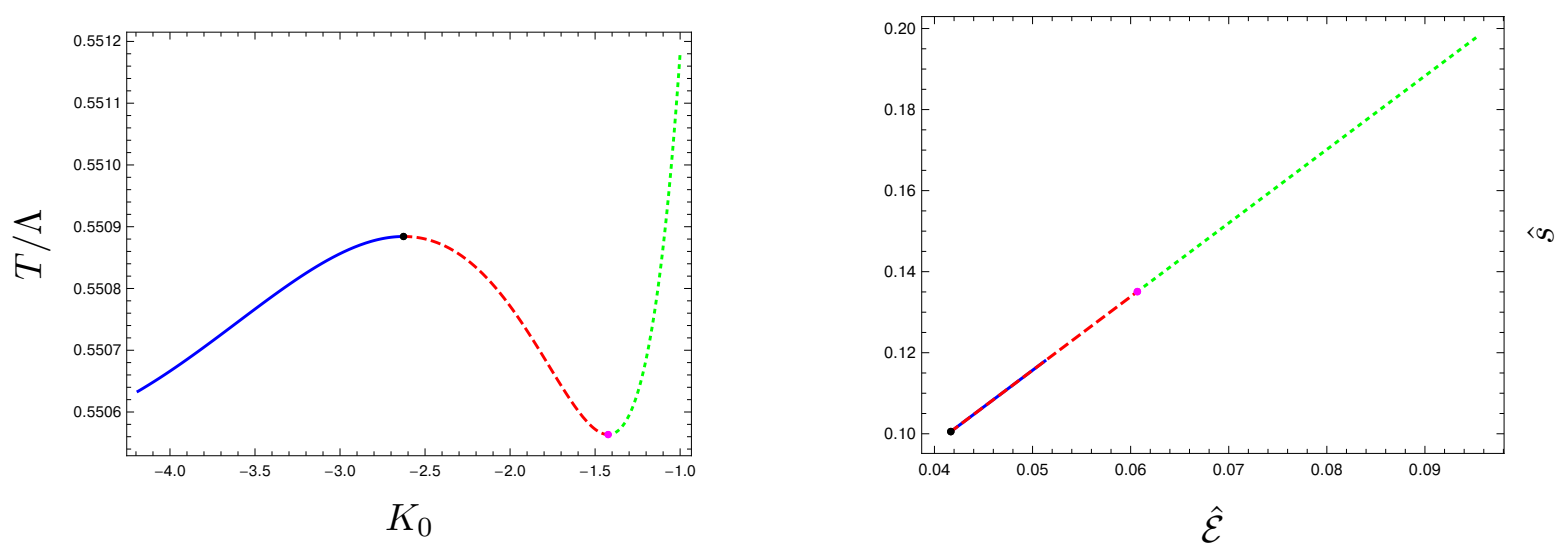

Figure 36. Physical observables of conifold black holes, here the Klebanov-Tseytlin black hole at $\mu=\mu_{2}$ (6.18), can depend on the numerical parameters ( $K_{0}$ in the left panel) non-monotonically. Some of this non-monotonicity, when translated to physical observables as in the right panel, results in retracing the same states of the black hole: the blue solid curve folds onto the red dashed curve in the right panel.

In figure 36 we present an example of the 'accidental' numerical test. The results reported relate to the Klebanov-Tseytlin black hole, i.e., $\mathcal{T}_{\text {decon }}^{s}$ thermal state, at fixed $\mu / \Lambda=\mu_{2} / \Lambda$, see (6.18). We use numerical scheme with $P=1, g_{s}=1$. We vary $K_{0}$ and $\mu$ along the line (6.34) to enforce the condition $\mu / \Lambda=$ constant. The left panel presents the results for $T / \Lambda$ as a function of $K_{0}$, and the right panel presents $\hat{s}$ as a function of $\hat{\mathcal{E}}$. Notice that $T / \Lambda$ is not a monotonic function of $K_{0}$. One aspect of this non-monotonicity is actually physics: the magenta dot represents the terminal temperature $T_{u}\left(\mu_{2} / \Lambda\right)$ of the Klebanov-Tseytlin black hole separating the phases with the positive (dotted green curve) and negative (dashed red curve) specific heat in the canonical ensemble, ${ }^{36}$ see figure 29 . On the contrary, the non-monotonicity highlighted by the black dot is a numerical artifact, akin to 'folding' for $b>1$ discussed above - the part of the blue curve retracing the same values of $T / \Lambda$ as those to the right of the black dot, represented by the red dashed curve, 'folds on itself' when we use the dimensionless physical observables, see the right panel. We did not show it, but the same 'folding' is true for the plot of $\hat{\mathcal{F}}$ as a function of $T / \Lambda$. What is different in this folding, compare to the one discussed above, is that it could not have been foreseen.

A similar example of the accidental numerical test, due to unforeseen 'folding' is highlighted in figure 33 .

\section{E.3 First law of thermodynamics of near-conformal conifold black holes}

In section 6.4 we discuss construction of $\mathcal{T}_{\text {decon }}^{s}$ thermal states, i.e., the Klebanov-Tseytlin black holes, perturbatively in $z$ (see (6.52) and (6.57))

$$
1 \gg z \equiv \frac{P^{2} g_{s}}{\hat{K}_{0}} \sim \frac{1}{2 \ln \frac{\mu}{\Lambda}}
$$

\footnotetext{
${ }^{36}$ Of course, this feature is invisible in the microcanonical ensemble, see the right panel of figure 36.
} 


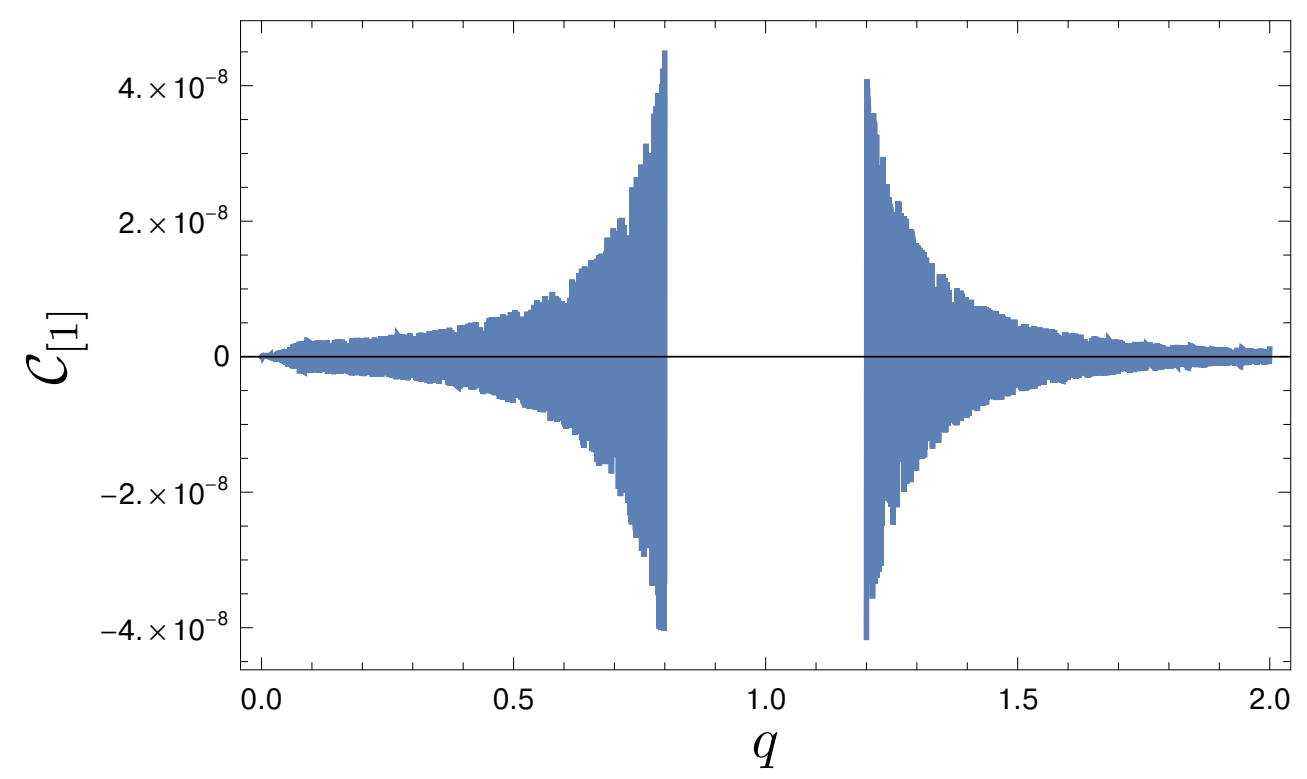

Figure 37. Verification of the first law of thermodynamics - see (6.66) for a precise definition of $\mathcal{C}_{[1]}$ - for the Klebanov-Tseytlin black holes to leading order in $1 / \ln (\mu / \Lambda)$ as a function of $q$, equivalently $\frac{T}{\mu}$ (E.6).

The first law of thermodynamics in this case leads to a series of differential constraints

$$
0 \equiv \sum_{n=0}^{\infty} z^{n} \mathcal{F} \mathcal{L}_{[n]}(q) \quad \Longrightarrow \quad \mathcal{F} \mathcal{L}_{[n]}(q)=0
$$

on parameters specifying the perturbative functions $f_{n}, f_{3, n}, f_{c, n}, h_{n}, k_{1, n}, g_{n}$. Explicit expression for $\mathcal{F} \mathcal{L}_{[1]}$ is given by (6.64) and (6.65). In figure 37 we verify the first law of thermodynamics at order $n=1-$ see (6.66) for a precise definition of $\mathcal{C}_{[1]}$. We excluded the region around $q=1$, as in this limit some of the parameters diverge - see figure 34 . The results are presented as a function of $q$; to leading order in $z$, see (6.63),

$$
\frac{T}{\mu}=\frac{q+2}{2 \pi q^{1 / 2}}(1+\mathcal{O}(z)) .
$$

\section{E.4 Additional numerical tests}

We list here additional some implicit and explicit numerical tests.

In multiple cases we cross checked numerics using different computational schemes, see figures 10 and 11.

Black branes on the conifold are $\mu / \Lambda \rightarrow 0$ limiting cases of the black holes discussed here. Even in this limit, the computations performed here differ from analysis in $[3,4,11]$. Specifically, in the latter work a different radial coordinate was used, see section 6.3. In all cases we found a perfect agreement in the $\mu / \Lambda \rightarrow 0$ limit with the results reported previously. 
Open Access. This article is distributed under the terms of the Creative Commons Attribution License (CC-BY 4.0), which permits any use, distribution and reproduction in any medium, provided the original author(s) and source are credited.

\section{References}

[1] I.R. Klebanov and A.A. Tseytlin, Gravity duals of supersymmetric $\mathrm{SU}(N) \times \mathrm{SU}(N+M)$ gauge theories, Nucl. Phys. B 578 (2000) 123 [hep-th/0002159] [INSPIRE].

[2] I.R. Klebanov and M.J. Strassler, Supergravity and a confining gauge theory: duality cascades and $\chi S B$ resolution of naked singularities, JHEP 08 (2000) 052 [hep-th/0007191] [INSPIRE].

[3] O. Aharony, A. Buchel and P. Kerner, The black hole in the throat: thermodynamics of strongly coupled cascading gauge theories, Phys. Rev. D 76 (2007) 086005 [arXiv:0706.1768] [INSPIRE].

[4] A. Buchel, Klebanov-Strassler black hole, JHEP 01 (2019) 207 [arXiv:1809.08484] [INSPIRE].

[5] J.M. Maldacena, The large $N$ limit of superconformal field theories and supergravity, Int. J. Theor. Phys. 38 (1999) 1113 [hep-th/9711200] [InSPIRE].

[6] O. Aharony, S.S. Gubser, J.M. Maldacena, H. Ooguri and Y. Oz, Large N field theories, string theory and gravity, Phys. Rept. 323 (2000) 183 [hep-th/9905111] [InSPIRE].

[7] E. Witten, Anti-de Sitter space, thermal phase transition, and confinement in gauge theories, Adv. Theor. Math. Phys. 2 (1998) 505 [hep-th/9803131] [InSPIRE].

[8] S.W. Hawking and D.N. Page, Thermodynamics of black holes in anti-de Sitter space, Commun. Math. Phys. 87 (1983) 577 [inSPIRE].

[9] P. Candelas and X.C. de la Ossa, Comments on conifolds, Nucl. Phys. B 342 (1990) 246 [INSPIRE].

[10] A. Buchel, Quantum phase transitions in cascading gauge theory, Nucl. Phys. B 856 (2012) 278 [arXiv:1108.6070] [INSPIRE].

[11] A. Buchel, Chiral symmetry breaking in cascading gauge theory plasma, Nucl. Phys. B 847 (2011) 297 [arXiv:1012.2404] [InSPIRE].

[12] I.R. Klebanov and E. Witten, Superconformal field theory on three-branes at a Calabi-Yau singularity, Nucl. Phys. B 536 (1998) 199 [hep-th/9807080] [INSPIRE].

[13] C.P. Herzog, I.R. Klebanov and P. Ouyang, Remarks on the warped deformed conifold, in Modern trends in string theory: $2^{\text {nd }}$ Lisbon school on $g$ theory superstrings, (2001) [hep-th/0108101] [INSPIRE].

[14] I. Bena, A. Buchel and S. Lüst, Throat destabilization (for profit and for fun),

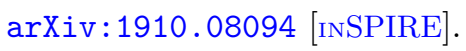

[15] A. Buchel, $\chi S B$ of cascading gauge theory in de Sitter, JHEP 05 (2020) 035 [arXiv: 1912.03566] [INSPIRE].

[16] B. Assel, D. Cassani, L. Di Pietro, Z. Komargodski, J. Lorenzen and D. Martelli, The Casimir energy in curved space and its supersymmetric counterpart, JHEP 07 (2015) 043 [arXiv: 1503.05537] [INSPIRE]. 
[17] M.R. Douglas and G.W. Moore, D-branes, quivers, and ALE instantons, hep-th/9603167 [INSPIRE].

[18] S. Kachru and E. Silverstein, $4 D$ conformal theories and strings on orbifolds, Phys. Rev. Lett. 80 (1998) 4855 [hep-th/9802183] [INSPIRE].

[19] Y. Tachikawa and B. Wecht, Explanation of the central charge ratio 27/32 in four-dimensional renormalization group flows between superconformal theories, Phys. Rev. Lett. 103 (2009) 061601 [arXiv:0906.0965] [inSPIRE].

[20] R. Minasian and D. Tsimpis, On the geometry of nontrivially embedded branes, Nucl. Phys. B 572 (2000) 499 [hep-th/9911042] [INSPIRE].

[21] S.S. Gubser, Einstein manifolds and conformal field theories, Phys. Rev. D 59 (1999) 025006 [hep-th/9807164] [INSPIRE].

[22] N. Seiberg, Electric-magnetic duality in supersymmetric non-Abelian gauge theories, Nucl. Phys. B 435 (1995) 129 [hep-th/9411149] [INSPIRE].

[23] A. Buchel, Finite temperature resolution of the Klebanov-Tseytlin singularity, Nucl. Phys. B 600 (2001) 219 [hep-th/0011146] [INSPIRE].

[24] A. Dymarsky, I.R. Klebanov and N. Seiberg, On the moduli space of the cascading $\mathrm{SU}(M+p) \times \mathrm{SU}(P)$ gauge theory, JHEP 01 (2006) 155 [hep-th/0511254] [INSPIRE].

[25] O. Aharony, A. Buchel and A. Yarom, Holographic renormalization of cascading gauge theories, Phys. Rev. D 72 (2005) 066003 [hep-th/0506002] [INSPIRE].

[26] V. Balasubramanian and P. Kraus, A stress tensor for anti-de Sitter gravity, Commun. Math. Phys. 208 (1999) 413 [hep-th/9902121] [INSPIRE].

[27] T. Prestidge, Dynamic and thermodynamic stability and negative modes in Schwarzschild-anti-de Sitter, Phys. Rev. D 61 (2000) 084002 [hep-th/9907163] [InSPIRE].

[28] V.E. Hubeny and M. Rangamani, Unstable horizons, JHEP 05 (2002) 027 [hep-th/0202189] [INSPIRE].

[29] A. Buchel, Universality of small black hole instability in AdS/CFT, Int. J. Mod. Phys. D 26 (2017) 1750140 [arXiv:1509.07780] [InSPIRE].

[30] A. Buchel and C. Pagnutti, Exotic hairy black holes, Nucl. Phys. B 824 (2010) 85 [arXiv:0904.1716] [INSPIRE].

[31] P. Bosch, A. Buchel and L. Lehner, Unstable horizons and singularity development in holography, JHEP 07 (2017) 135 [arXiv:1704.05454] [INSPIRE].

[32] A. Buchel, Singularity development and supersymmetry in holography, JHEP 08 (2017) 134 [arXiv: 1705.08560] [INSPIRE].

[33] A. Buchel, Thermal order in holographic CFTs and no-hair theorem violation in black branes, Nucl. Phys. B 967 (2021) 115425 [arXiv: 2005.07833] [INSPIRE].

[34] A. Buchel, Fate of the conformal order, Phys. Rev. D 103 (2021) 026008 [arXiv: 2011.11509] [INSPIRE].

[35] A. Buchel, Holographic conformal order in supergravity, Phys. Lett. B 814 (2021) 136111 [arXiv: 2007.09420].

[36] N. Chai, S. Chaudhuri, C. Choi, Z. Komargodski, E. Rabinovici and M. Smolkin, Thermal order in conformal theories, Phys. Rev. D 102 (2020) 065014 [arXiv:2005.03676] [INSPIRE]. 
[37] A. Buchel, C.P. Herzog, I.R. Klebanov, L.A. Pando Zayas and A.A. Tseytlin, Nonextremal gravity duals for fractional D3 branes on the conifold, JHEP 04 (2001) 033 [hep-th/0102105] [INSPIRE].

[38] S.S. Gubser, C.P. Herzog, I.R. Klebanov and A.A. Tseytlin, Restoration of chiral symmetry: a supergravity perspective, JHEP 05 (2001) 028 [hep-th/0102172] [INSPIRE].

[39] A. Buchel, Hydrodynamics of the cascading plasma, Nucl. Phys. B $\mathbf{8 2 0}$ (2009) 385 [arXiv:0903.3605] [INSPIRE].

[40] A. Buchel, On branches of the KS black hole, Nucl. Phys. B 957 (2020) 115094 [arXiv:2005.00854].

[41] A. Buchel, A holographic perspective on Gubser-Mitra conjecture, Nucl. Phys. B 731 (2005) 109 [hep-th/0507275] [INSPIRE].

[42] A. Buchel and C. Pagnutti, Bulk viscosity of $N=2 *$ plasma, Nucl. Phys. B 816 (2009) 62 [arXiv: 0812.3623] [INSPIRE].

[43] A. Buchel, Stability of Klebanov-Strassler black holes, work in progress.

[44] A. Buchel, Black hole spectra in holography: consequences for equilibration of dual gauge theories, Nucl. Phys. B 896 (2015) 587 [arXiv:1501. 04593] [INSPIRE].

[45] A. Buchel and M. Buchel, On stability of nonthermal states in strongly coupled gauge theories, arXiv: 1509.00774 [INSPIRE].

[46] A. Buchel and B. Umbert, QGP universality in a magnetic field?, JHEP 06 (2020) 149 [arXiv: 2004.01583] [INSPIRE].

[47] D. Cassani and A.F. Faedo, A supersymmetric consistent truncation for conifold solutions, Nucl. Phys. B 843 (2011) 455 [arXiv:1008.0883] [INSPIRE].

[48] A. Buchel, Effective action of the baryonic branch in string theory flux throats, JHEP 09 (2014) 117 [arXiv:1405.1518] [INSPIRE]. 